\title{
Genetic analysis of stoned B/stonin 2 function in vivo
}

\section{PhD Thesis}

\author{
in partial fulfilment of the requirements \\ for the degree "Doctor of Philosophy (PhD)" \\ in the Molecular Biology Program \\ at the Georg August University Göttingen, \\ Faculty of Biology
}

submitted by

Muhammed Kasim Diril

born in

Canakkale, Turkey

Göttingen 2005 


\section{Affidavit}

Here I declare that my Ph.D. thesis entitled "Genetic analysis of stoned B/stonin 2 function in vivo" has been written independently and with no other sources and aids then quoted.

Muhammed Kasim Diril

May $30^{\text {th }}, 2005$

Berlin 


\section{ABSTRACT}

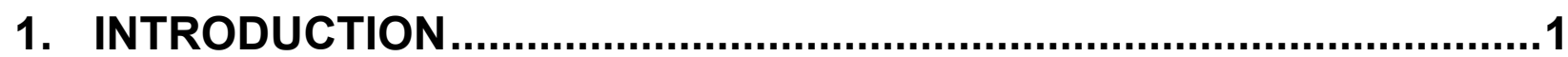

1.1 Compartmentalization of cells and vesicular trafficking .......................... 1

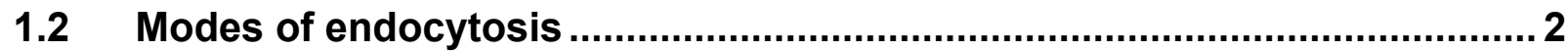

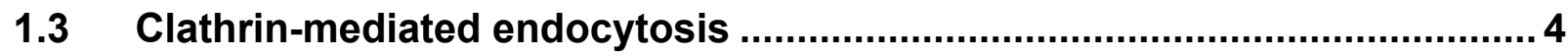

1.3.1 Sequence of events in clathrin-mediated endocytosis .............................. 5

1.4 Components of the endocytic machinery .................................................

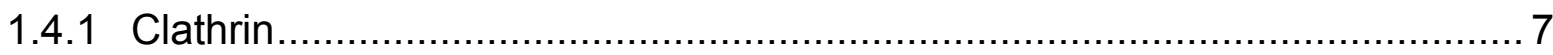

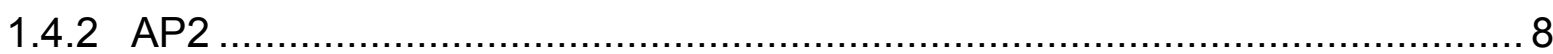

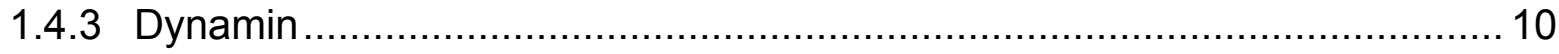

1.4.4 Other accessory proteins and protein-protein interaction networks............. 10

1.5 Synaptic vesicle cycle and endocytosis................................................12

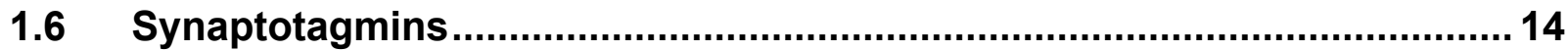

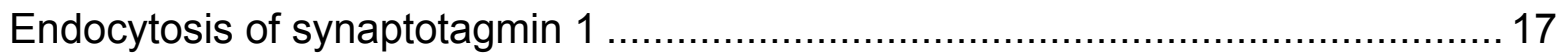

1.7 The Drosophila stoned mutation and its phenotypic characterization ..... 17

1.8 Sequence analysis of stoned and stonin genes …....................................20

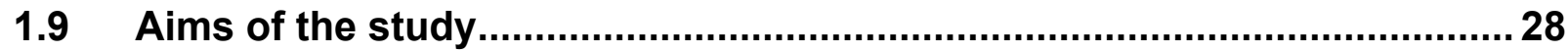

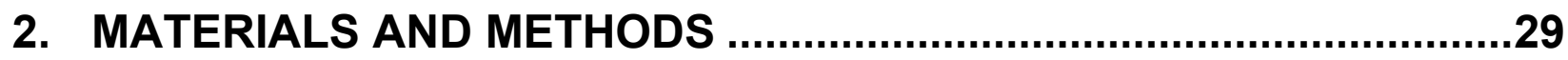

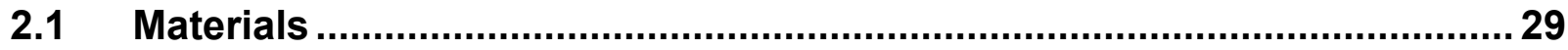

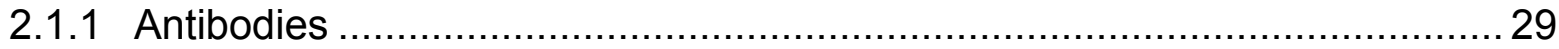

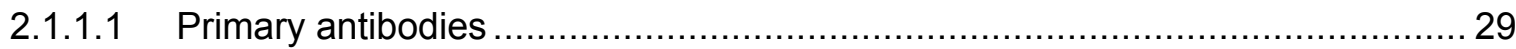

2.1.1.2 Secondary antibodies ........................................................................... 30

2.1.2 Oligonucleotide primers and siRNAs................................................ 30

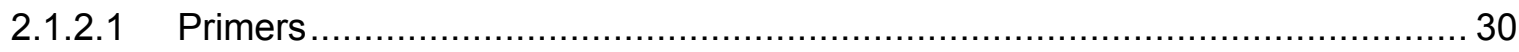

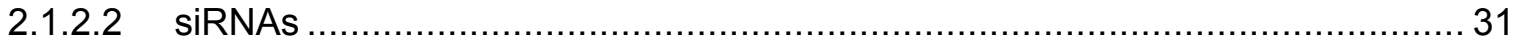

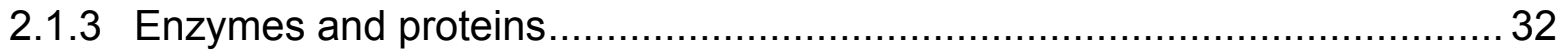

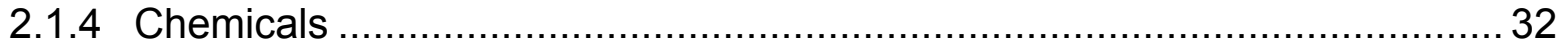

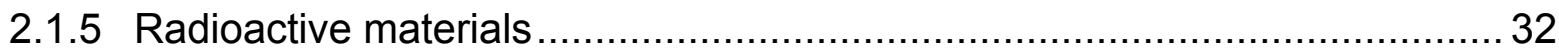

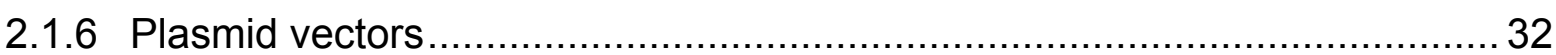

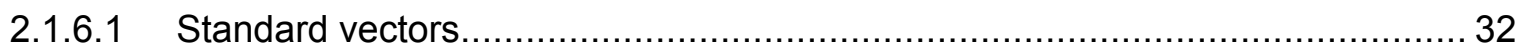

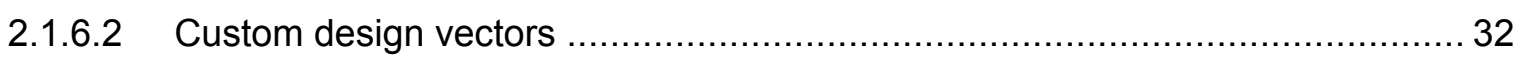




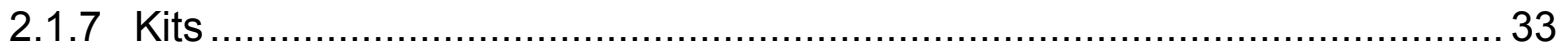

2.1.8 Commonly used software and web interfaces...................................... 34

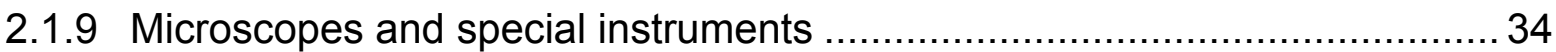

2.1.10 Bacterial strains and mammalian cell lines ............................................. 34

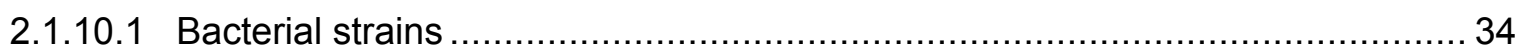

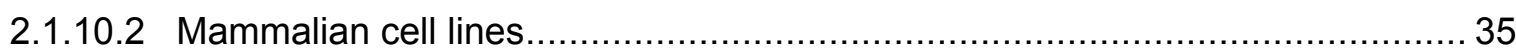

2.1.11 Commonly used buffers, solutions and media ...........................................35

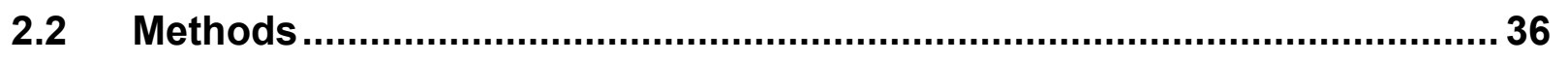

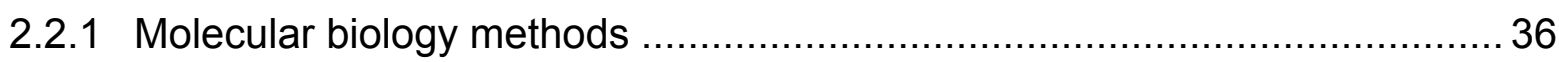

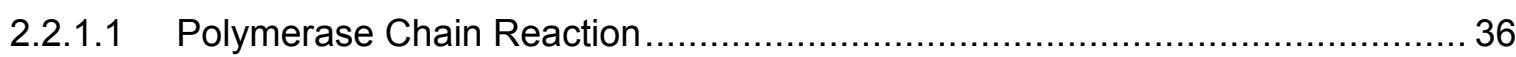

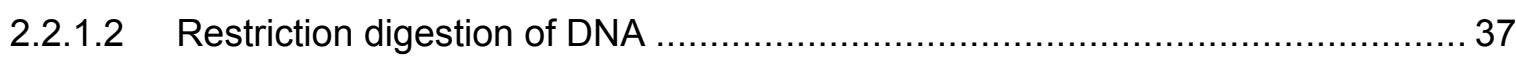

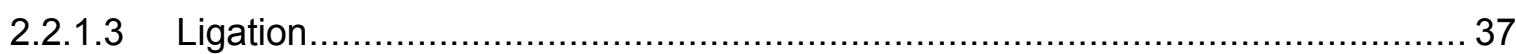

2.2.1.4 Transformation of chemically competent E.Coli .......................................... 38

2.2.2 Expression and purification of recombinant proteins ................................ 38

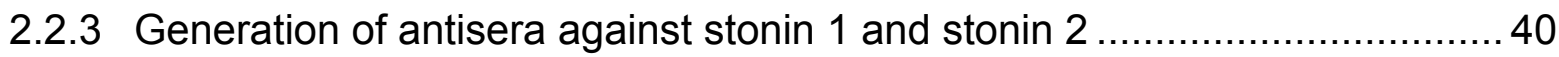

2.2.4 Preparation of genomic DNA from cells and animal tissue ...................... 42

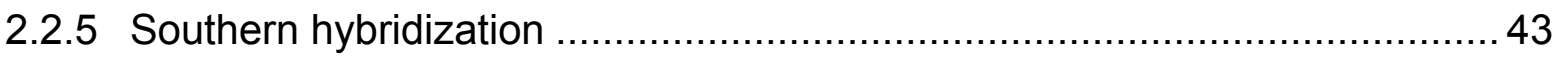

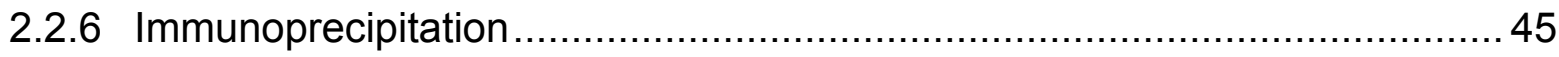

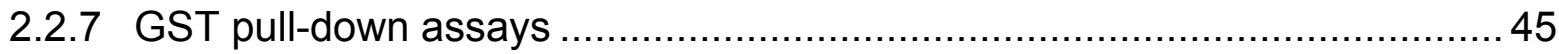

2.2.8 SDS-PAGE

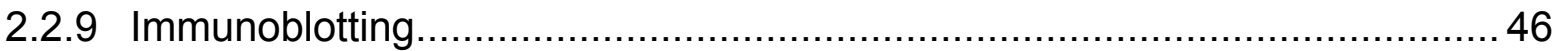

2.2.10 Mouse ES cell culture and electroporation of the targeting vector .............. 47

2.2.11 Primary mouse embryonic fibroblasts culture and mitotic inactivation .........50

2.2.12 Construction of stonin 2 locus targeting vector ...................................... 51

2.2.13 Screening of embryonic stem cell colonies and microinjection ................... 54

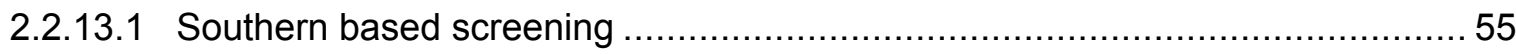

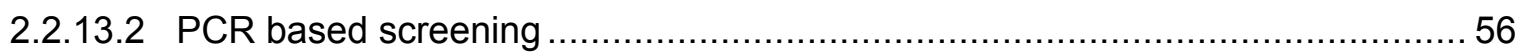

2.2.13.3 Microinjection of positive ES cell colonies and generation of chimera .............57

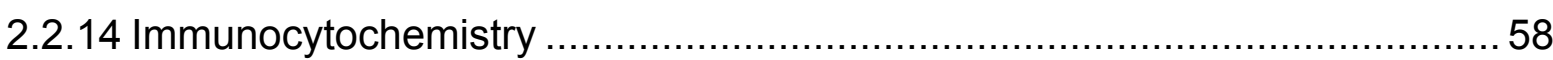

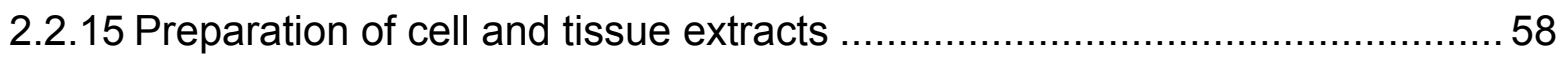

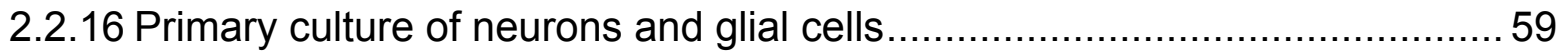

2.2.17 Eukaryotic cell culture, transfections and creation of stable cell lines ..........60

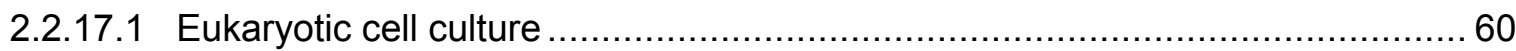

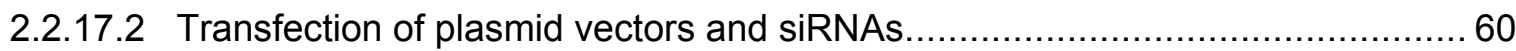

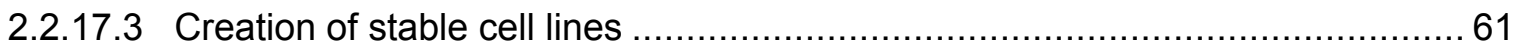


2.2.18 Antibody internalization assays and quantification ............................... 62

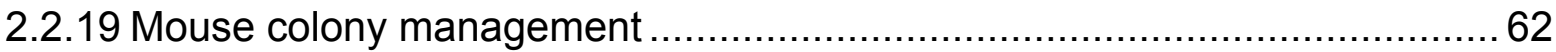

3. RESULTS.................................................................................64

3.1 Tissue distribution and intracellular localization of stonin $2 \ldots \ldots \ldots \ldots \ldots \ldots . . . . . . . . . . .64$

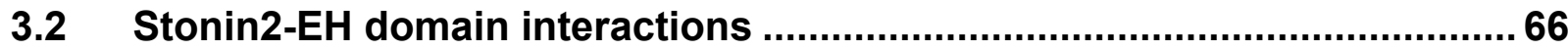

3.3 Stonin 2 binds directly to AP2 via a novel type of $\alpha$-ear binding motif..... 68

3.4 Overexpression of stonin2 but not stonin1 blocks CME in COS7 cells.... 72

3.5 Analysis of the stonin-synaptotagmin interactions................................ 74

3.6 Stonin 2 facilitates AP2 dependent endocytosis of synaptotagmin $1 \ldots . . .80$

3.7 Sorting of synaptotagmin 1 following endocytosis ................................ 94

3.8 Analysis of stonin2 deficient mice ..................................................... 98

4. DISCUSSION AND CONCLUSIONS ..........................................110

4.1 Stonin 2: an alternate sorting adaptor in CME .................................... 110

4.2 Stonin 2 in synaptotagmin 1 endocytosis and sorting........................... 114

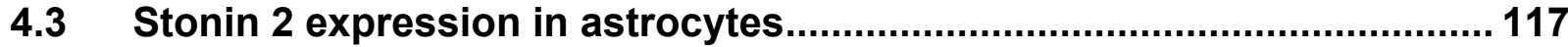

$4.4 \quad$ Analysis of stonin 2 knock-out mice........................................................ 117

4.5 Summary and Conclusions ................................................................. 119

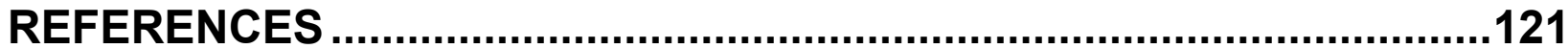

LIST OF FIGURES AND TABLES ..................................................130

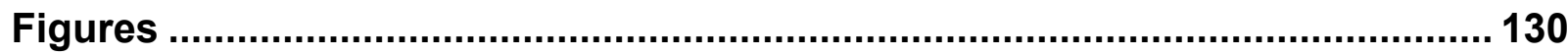

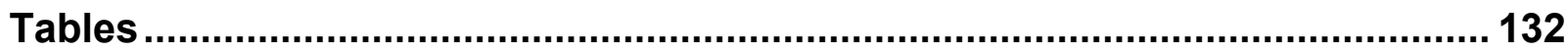

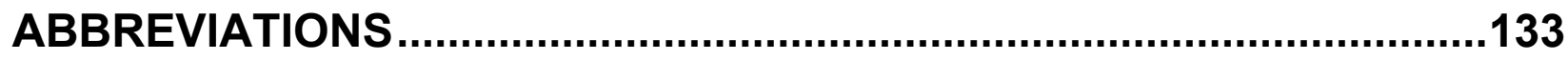

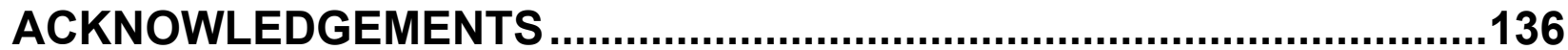

CURRICULUM VITAE...........................................................................137

PUBLICATIONS...........................................................................138 


\begin{abstract}
Clathrin-mediated endocytosis at presynaptic nerve terminals is a regulated process tightly coupled to the exocytotic fusion event that requires clathrin, dynamin, AP2 as well as synaptotagmin 1 . Stonin 2 is a brain-enriched protein which localizes to axonal vesicle clusters in neurons and clathin/AP2-coated pits in astroglial cells. Its Drosophila orthologue stoned B has a putative role in synaptic vesicle recycling, especially sorting and endocytosis of synaptotagmins. Mammalian stonin 2 interacts with the heterotetrameric clathrin adaptor AP2, via three novel WVXF motifs, and with other endocytic accessory proteins eps 15 and intersectin 1 by virtue of its two NPF motifs in its amino-terminal domain. The carboxy-terminal $\mu \mathrm{HD}$ binds to synaptotagmin 1 and is required for proper localization of stonin 2 to synapses in neurons and to coated-pits in astroglial cells. We have recently developed an in vivo interaction assay, to test the interactions between stonins and synaptotagmin family members (1-11). Predominantly cytosolic stonin 2 , translocates to the plasmalemma and colocalizes with AP2-coated pits when co-expressed together with synaptotagmins 1, 2, and 9. Transgenic expression of stonin 2 in a stable HEK293 cell line expressing lumenally FLAG-tagged synaptotagmin 1, stimulates endocytic internalization of plasma membrane-stranded synaptotagmin 1 more than 20-fold. Stonin 2 thus physically links synaptotagmin 1 and AP2 and this linkage is required for synaptotagmin 1 endocytosis. Disruption of the stonin 2-AP2 interaction by mutagenesis of WVXF motifs or RNAi-mediated silencing of AP2 expression, inhibits stonin 2-assisted synaptotagmin 1 uptake. In neuroendocrine PC12 cells, stonin 2 assists in targeting of internalized synaptotagmin 1 to SLMVs in addition to facilitating its endocytosis. For a better characterization of its functions, a stonin 2-deficient mouse strain was created by targeted disruption of the stonin 2 gene. Stonin 2 knock-out animals are viable and fertile. Preliminary analysis of synaptotagmin 1 distribution, endocytic recycling, number and morphology of synaptic vesicles in stonin 2 deficient nerve terminals did not display any abnormalities compared to wildtype littermates. Therefore refined experimental procedures seem to be required for characterization of stonin 2 deficiency in neurons and astrocytes. We propose that stonin 2 is an endocytic sorting adaptor for clathrin-mediated endocytosis of synaptotagmins in neurons and neurosecretory cells, as well as yet unidentified cargo molecule(s) in astroglial cells.
\end{abstract}




\section{INTRODUCTION}

\subsection{Compartmentalization of cells and vesicular trafficking}

One of the most prominent aspects that distinguishes eukaryotic cells from prokaryotic cells is the presence of a vast variety of intracellular membrane-encircled compartments. Evolution of these intracellular compartments and organelles has provided eukaryotic cells with the ability to execute an enormous array of different processes within the body and to specialize in diverse functions. The existence of intracellular compartments with different functions and distinct compositions of membrane proteins and lipids to perform those functions, introduces a challenge, which prokaryotic cells do not have to face (at least not to the same extent): the creation and preservation of the compartmental identity. Eukaryotes overcome this challenge by means of complex mechanisms of membrane trafficking which involves the sorting of proteins and lipids to distinct membrane domains, the generation of carrier vesicles or tubules from larger, membrane-bound organelles and transport and fusion of these vesicles with target membranes (Munro, 2004; McMahon and Mills, 2004; Lodish et al., 2000; Alberts et al., 2002).

Three major kinds of transport vesicles have been identified within eukaryotic cells, which are classified according to the coat protein components required for their biogenesis. Reversible polymerization of coat proteins on the planar, cytosolic surface of larger membranes introduces membrane curvature which is needed to eventually pinch off transport vesicles (McMahon and Mills,2004; Brodsky et al., 2001). Each type of transport vesicle carries proteins from a particular organelle of origin to another destination organelle (i.e. transport of secreted proteins from the endoplasmic reticulum to the cis-Golgi complex). Cargo proteins generally possess short sorting signals that are recognized by adapter proteins (Bonifacino and Traub, 2003), which link cargo with other coat components (Traub, 2003; Boehm and Bonifacino, 2001).

COPII coated vesicles are generated at rough endoplasmic reticulum membrane, and they transport secreted and transmembrane proteins in the anterograde direction and finally fuse with the cis-Golgi or ERGIC (endoplasmic reticulum-Golgi intermediate compartment) compartments. COPI coated vesicles form at the Golgi cisternae and are involved in retrograde trafficking of proteins in trans-Golgi to cis- 
Golgi or cis-Golgi to endoplasmic reticulum direction. Finally, clathrin coated vesicles are created at the plasma membrane or the trans-Golgi network and carry proteins destined for endosomes and lysosomes (Figure 1.1).

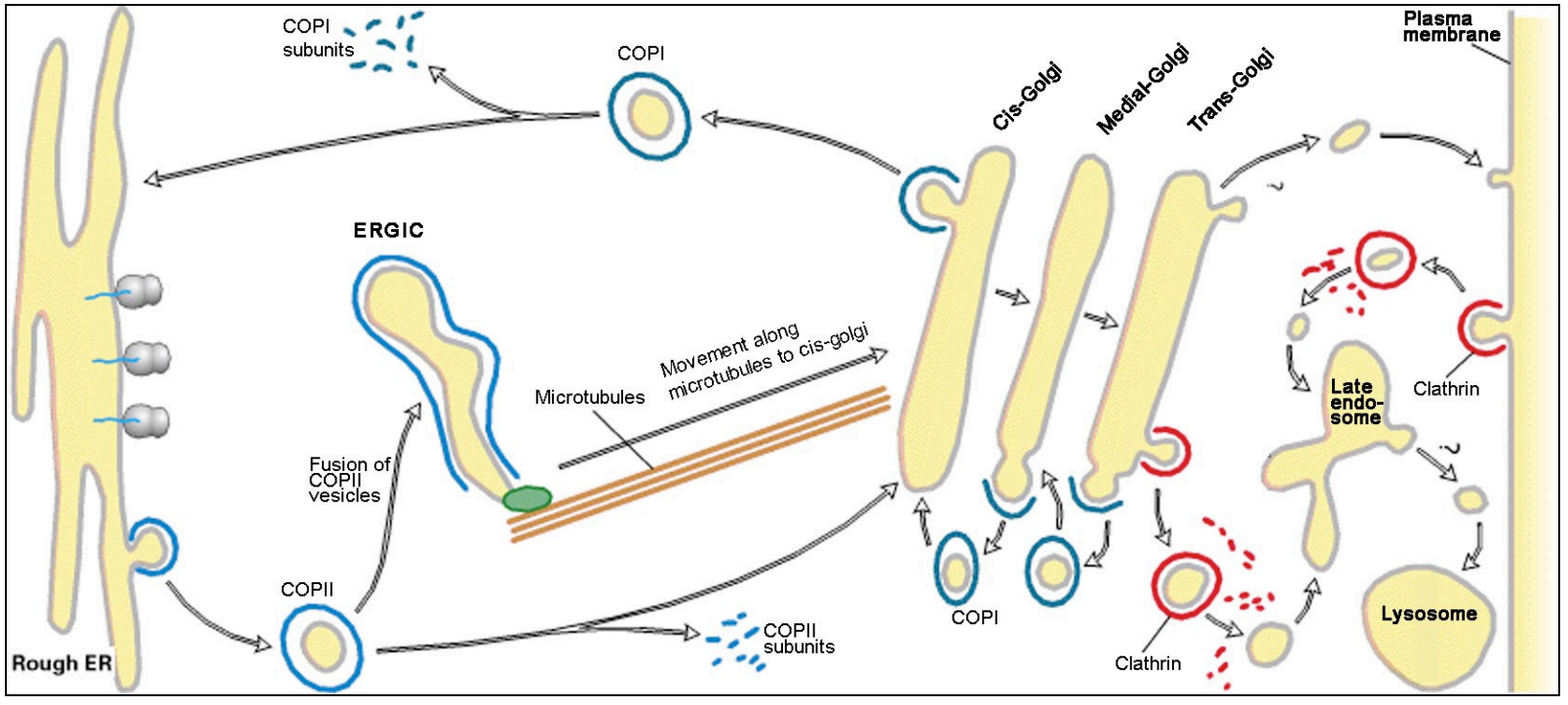

Figure 1.1 Diverse intracellular vesicular trafficking events in eukaryotic cells. Coat proteins assist in the budding of transport vesicles from their organelle of origin. After the vesicles are pinched off, they shed their coats by the help of uncoating factors and fuse with their destination membranes. Depolymerized coat components can be used for a new round of vesicle biogenesis. COPII-coated vesicles bud off from the rough ER membrane and transport proteins in the anterograde direction to the cis-Golgi. COPI-coated vesicles are generated from the Golgi stacks and participate in transport of proteins in the retrograde direction within the Golgi and from the cis-Golgi to the ER. Vesicles generated from the plasma membrane and trans-Golgi network by clathrin coats, fuse with endosomes after shedding their coat. (adapted from Lodish et al., 2000, Molecular Cell Biology, $4^{\text {th }}$ Ed.)

\subsection{Modes of endocytosis}

The plasma membrane does not only serve as a selective permeability barrier that separates the cytoplasm from the extra-cellular environment, but also acts as an active interface for uptake of nutrients and communication of cells with their environment. Endocytosis is the collective name of several cellular processes whereby cells internalize extra-cellular molecules and particles in membrane bound vesicles generated from the plasma membrane. Two major types of endocytosis occur in eukaryotic cells; phagocytosis ('cell eating', uptake of large particles i.e. bacteria) and pinocytosis ('cell drinking' or fluid phase uptake). Pinocytosis can be further divided into three categories; i) macropinocytosis, ii) caveolae-mediated endocytosis iii) endocytosis by lipid rafts and membrane microdomains, and iv) clathrin-mediated endocytosis (Figure 1.2), (Conner and Schmid, 2003). 


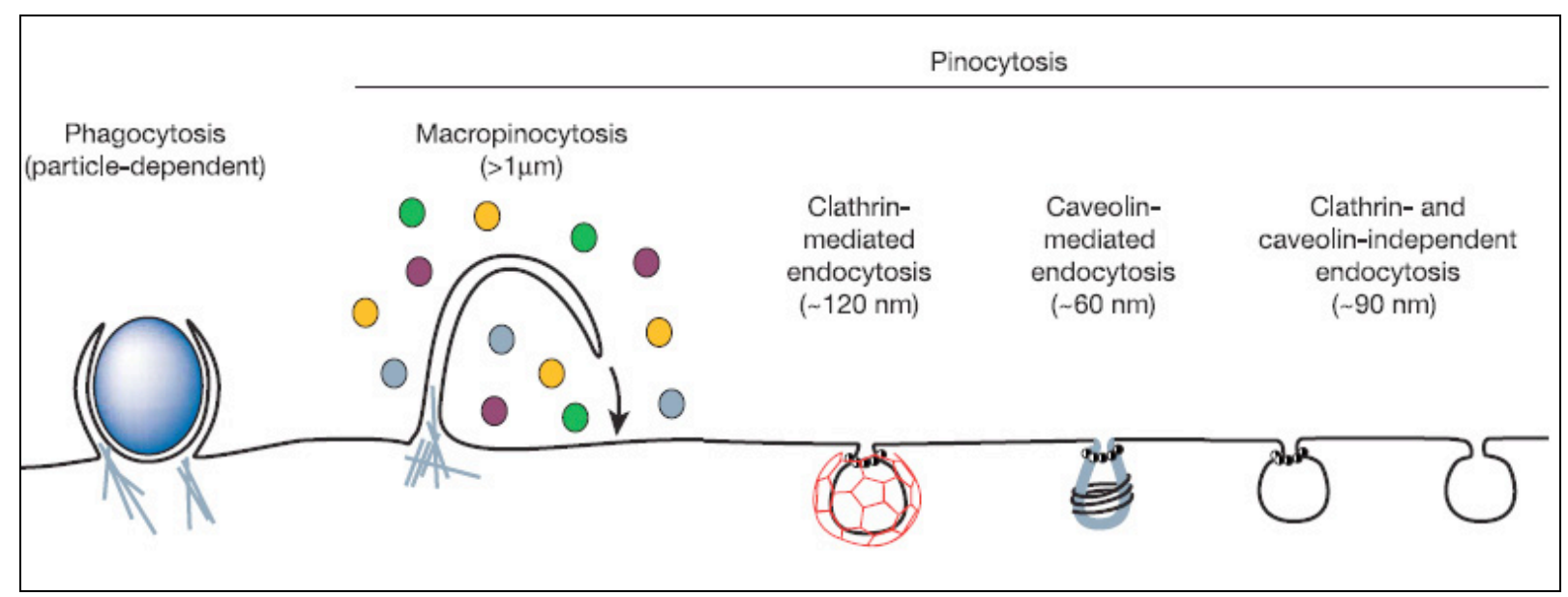

Figure 1.2 Routes of entry into eukaryotic cells. Summary of the different endocytosis pathways utilized by eukaryotic cells to internalize a variety of cargo. Note that, not all mechanisms are available to every cell type. The size of the endocytosed vesicles also varies greatly according to the nature of the cargo. (excerpted from Conner and Schmid, 2003)

Phagocytosis, the primary mechanism of clearing the extracellular milieu from pathogenic microorganisms as well as remains of dead cells and aggregates, can be performed by only a subset of cells including immune system cells. It is achieved by the encapsulation of foreign bodies by plasma membrane protrusions and directed by remodeling of the actin cytoskeleton (Conner and Schmid, 2003).

Macropinocytosis is similar to phagocytosis in the underlying signaling cascades which involve Rho family GTPases, but plasma membrane protrusions collapse back on the surface of the cells to generate large endocytic vesicles called macropinosomes. Both phagocytosis and macropinocytosis can be hijacked by some microorganisms allowing their entry into eukaryotic cells (Conner and Schmid, 2003).

Caveolae-mediated endocytosis is used predominantly by endothelial cells in transcytosis of extracellular ligands. Caveolae are flask-shaped plasma membrane invaginations that are rich in cholesterol and sphingolipids, and they are created by membrane-associated proteins called caveolins. Disruption of the caveolin 1 gene in mice results in total disappearance of otherwise abundant caveolae from endothelial cell plasma membranes, but the animals are viable (Drab et al., 2001). Caveolaemediated uptake is slower than clathrin-mediated endocytosis (CME) with half times greater than 20 minutes. The internalized vesicles have a diameter of about 50 $60 \mathrm{~nm}$. As in CME, dynamin is essential for vesicle scission event (Johannes, 2002).

Clathrin-mediated endocytosis is carried out by all types of mammalian cells and is essential for uptake of nutrients like LDL or transferrin as well as signalling receptors 
from the cell surface. During CME, transmembrane cargo proteins are concentrated within specialized regions of the plasma membrane by the help of AP2 adaptor proteins, which promote the assembly of clathrin-coated pits (Schmid, 1997; Brodsky, 2001; Haucke and Krauss, 2002). CME is also a widely accepted mechanism for the regeneration of synaptic vesicles in presynaptic nerve terminals following neurotransmitter release (Cremona and De Camilli, 1997; Galli and Haucke, 2001 and 2004; Slepnev and De Camilli, 2000). Detailed information about CME will be presented in the next section.

Inhibition of CME by dominant-negative eps15 mutants does not have any effect on internalization of some membrane proteins such as the IL-2 receptor in lymphocytes (Conner and Schmid, 2003). Indeed, there are also clathrin- and caveolinindependent endocytic pathways, which are mediated by concentration of cargo proteins in plasma membrane lipid rafts or special membrane microdomains (Johannes, 2002).

\subsection{Clathrin-mediated endocytosis}

Clathrin-coated pits (CCPs) and vesicles were initially visualized as bristle coated vesicles and membranes during the electron microscopical analysis of mosquito oocytes taking up extracellular yolk proteins (Roth and Porter, 1964). During the forthcoming four decades, major structural and functional components of these 'bristle' coated membrane structures have been identified one by one (Pearse, 1975; Keen et al., 1979), and the steps involved in early nucleation of coated pits to the final dissociation of the clathrin have been well characterized (Takei and Haucke, 2001 ).

CCPs assemble on cytosolic leaflets of the plasma membrane as well as the transGolgi network (TGN) membranes, and function in sorting of proteins into transport vesicles that eventually fuse with endosomes and lysosomes. A good analogy for sorting of cargo proteins to coated pits, generation of coated pits and fission of clathrin-coated vesicles would be an arm picking up some objects from the ground. According to this analogy clathrin can be compared to the bones and skeleton of the arm, as its major function is that of a structural scaffold for the uptake event. Adapter proteins including AP2 (or AP1 in case of budding from the TGN) that recognize and deliver the membrane cargo proteins to CCPs can be compared to the hand in the same analogy. Just as a hand needs fingers for fine adjustment and recognition of 
different objects, AP2 utilizes a plethora of accessory adapter proteins that recognize a variety of different cargo molecules. Finally, dynamin could be compared to the muscles, as its GTPase activity provides the energy for the final budding event. There are also other factors that are essential for CME (i.e. uncoating factors) which do not fit into this analogy.

\subsubsection{Sequence of events in clathrin-mediated endocytosis}

CME can be roughly divided into 4 steps; i) nucleation, ii) bud formation, iii) fission, and iv) uncoating (Figure 1.3).

Clathrin-coated pit nucleation is mediated by adapter proteins and other endocytic accessory proteins (i.e. epsin, AP180) which form a dynamic network of proteinprotein interactions. Endocytic accessory proteins recognize membrane cargo proteins using the sorting motifs present in their cytosolic tails (Bonifacino and Traub, 2003) or attached mono-ubiquitin residues (Aguilar and Wendland, 2005; Chen and De Camilli, 2005; Sigismund et al., 2005). Binding of adapter proteins to cargo molecules initiates coated pit nucleation. For instance, during synaptic vesicle endocytosis, binding of AP2 to peptides bearing tyrosine-based endocytic sorting motifs stimulates their interaction with synaptotagmin 1 (Haucke and De Camilli, 1999; Haucke et al., 2000).

Membrane lipids also serve an active role in the coat nucleation event. Endocytic accessory proteins like AP2, AP180 or CALM and epsin are recruited to the plasma membrane during endocytosis by binding to certain plasma membrane lipids, in particular to $\mathrm{PIP}_{2}$ (Phosphatidylinositol (4,5)-bisphosphate). Arf6, a small GTPase switch protein, can stimulate AP2 and clathrin recruitment to the plasma membrane via activation of PIPKl $\gamma$, the major $\mathrm{PIP}_{2}$ generating enzyme (Krauss et al., 2003). Clathrin is recruited to and assembles onto a network of endocytic accessory proteins bound to cargo molecules. During the initial stages of coated pit generation, the plasma membrane is still planar but coated with clathrin (Figure 4D).

During bud formation events, the plasma membrane acquires a positive curvature driven in part by the assembly of clathrin cages and adapter-mediated membrane bending (Ford et al., 2002). The mechanism by which planar clathrin-coated membranes are converted to coated buds is not very well understood, but may involve a change from a predominantly hexagonal organization of clathrin triskelia to 
one that includes 12 pentagons in the final coat. Purified clathrin can also be induced to form cage-like structures in the absence of membranes (Fotin et al., 2004).

Fission of clathrin-coated vesicles is achieved by dynamin which acts as a mechanochemical enzyme that drives membrane vesiculation. Dynamin is also required for other endocytic processes like phagocytosis, caveolin-dependent endocytosis and raft- or microdomain-mediated endocytosis. During the budding step, dynamin molecules are recruited to the neck of a clathrin coated pit and encircle it as a molecular collar. GTP hydrolysis causes the constriction and helical expansion of this collar and leads to fission of the vesicle from the plasma membrane.

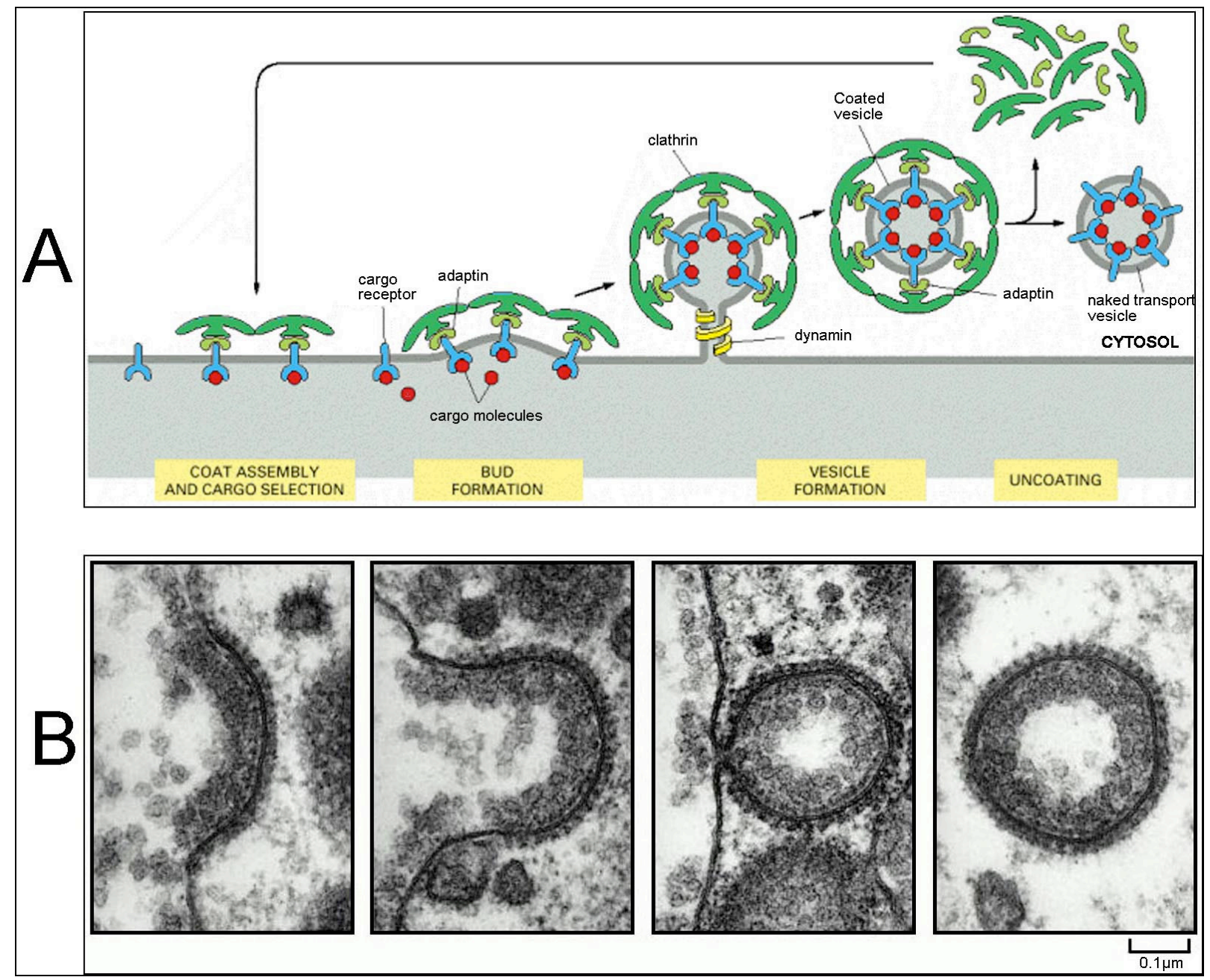

Figure 1.3 Sequence of events during clathrin mediated endocytosis. (A) Clathrin mediated endocytosis requires coated pit nucleation via selection of cargo proteins. A positive curvature is gradually introduced into the membrane to generate coated pits. GTP hydrolysis by dynamin supplies the energy for the fission event. Clathrin-coated vesicles are rapidly stripped off from their coats in the cytosol by the combined action of Hsc70 and auxilin (excerpted from Alberts et al., 2002, Molecular Biology of the Cell, $4^{\text {th }}$ Ed.). (B) One of the first electron microscope pictures showing clathrinmediated endocytosis. Oocytes of the hen utilize CME in uptake of lipoprotein particles. The lipoprotein can be seen as a fuzzy layer on the extracellular facet of the plasma membrane (left side). 
Note the CCVs generated as a result of this process are abnormally large in size compared to conventional CCVs. (Excerpted from Perry and Gilbert, 1979)

Uncoating of clathrin-coated vesicles is believed to be a rapid event compared to other steps. Therefore free clathrin-coated vesicles are rarely observed in electron micrographs of cells. The uncoating reaction involves the disassembly of the clathrin lattice encapsulating the transport vesicle. Hsc70, a 70kDa chaperone protein which belongs to the DnaK family of heat shock proteins, is targeted to clathrin-coated vesicles by auxilin. There it drives clathrin uncoating by providing energy via its ATPase function (Morgan et al., 2001; Takei and Haucke, 2001; Brodsky, 2001). Synaptojanin 1, a phosphoinositide phosphatase at presynaptic nerve terminals, is another essential factor for uncoating of internalized synaptic vesicles. Its targeted deletion results in accumulation of coated vesicles near periactive zones (Cremona et al., 1999).

\subsection{Components of the endocytic machinery}

Clathrin-mediated endocytosis is a highly regulated process that involves the coordinated action of many factors including clathrin, AP2, dynamin, uncoating factors, cargo proteins, membrane lipids, and various accessory factors. Brief descriptions of some of these factors will be presented here.

\subsubsection{Clathrin}

Clathrin coats are built by the regulated polymerization of clathrin heavy chain $(\mathrm{CHC})$ and clathrin light chain (CLC) on the plasma membrane. $\mathrm{CHC}$ is a large protein with $192 \mathrm{kDa}$ molecular weight. Three identical $\mathrm{CHC}$ molecules are organized into clathrin triskelia in solution (Figure 1.4). The C-terminal domain of the $\mathrm{CHC}$ is called the hub domain and constitutes the central part of the clathrin triskelia (Figure 1.4A). The Nterminal domain, which is also called $\beta$-propeller domain because of its structure, is globular and faces the interior of the clathrin cages. Many accessory proteins including AP1 and AP2 adaptor complexes bind to this domain by the help of the clathrin interaction motifs present in their sequences. The middle part of the $\mathrm{CHC}$ is a superhelix composed of $\alpha$-helices and forms the proximal and distal legs of the clathrin triskelia. CLC molecules bind to the triskelia at the central hub region and negatively regulate its polymerization at physiological $\mathrm{pH}$. Adaptor proteins AP1 and AP2 have the ability to relieve this inhibition and stimulate clathrin polymerization (Brodsky, 2001; Fotin et al., 2004). 


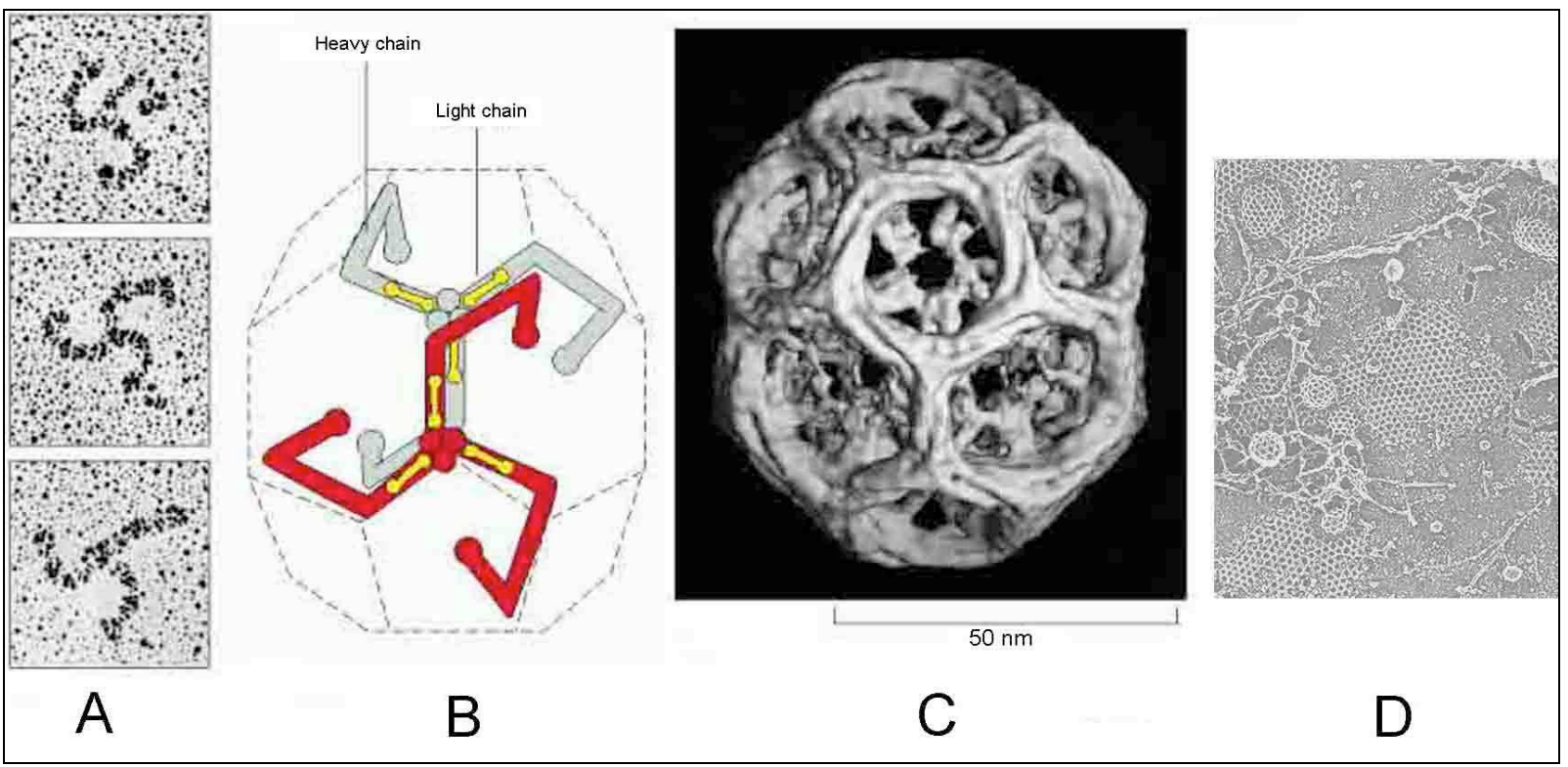

Figure 1.4 Molecular architecture and organization of the clathrin coat. (A) Electron micrographs of clathrin heavy chains assembled as triskelia. (Ungewickell and Branton, 1981) (B) Schematic drawing of the organization of clathrin triskelia within the clathrin coated vesicles. One of the $\mathrm{CHCs}$ are shown in red and CLCs are shown in yellow.N-terminal domain is bent inward where it could interact with other accessory factors during polymerization. (Nathke et al., 1992) (C) 3D structure of clathrin cages were obtained by cryo electron microscopy. 36 triskelions have been organized into 12 pentagons and 6 hexagons. The $\mathrm{N}$-terminal domains of the triskelions make an inner shell. (Smith et al., 1998) (D) Freeze fracture EM image of clathrin coated membranes and buds on the inner surface of the plasma membrane. (Courtesy of John Heuser, Washington University). (A, B and C were excerpted from Alberts et al., 2002, Molecular Biology of the Cell, $4^{\text {th }}$ Ed.)

\subsubsection{AP2}

AP2 is the most studied and best characterized member of the heterotetrameric clathrin adaptor proteins. Other members of this family include AP1, AP3 and AP4. AP1 acts at the TGN and plays an analogous role to that of AP2 in clathrin-mediated membrane budding (Boehm and Bonifacino, 2001). All four proteins have a similar subunit composition. In addition, crystal structures of the core and appendage domains of AP1 and AP2 are very similar (Collins et al., 2002; Heldwein et al., 2004).

Electron microscopical analysis of purified AP2 complexes showed that it is comprised of a large central brick-like core domain and two appendage domains attached to it via flexible linkers (Figure 1.5A). All AP complexes are made up of two large subunits of $100-130 \mathrm{kDa}$ size ( $\alpha$ and $\beta_{2}$ in case of AP2), a medium subunit with $50 \mathrm{kDa}$ size ( $\mu_{2}$ in AP2) and finally a small $17 \mathrm{kDa}$ subunit $\left(\sigma_{2}\right.$ in AP2). Amino-terminal domains of $\alpha$ and $\beta_{2}$ subunits (approximately $70 \mathrm{kDa}$ ) together with $\mu_{2}$ and $\sigma_{2}$ subunits form the trunk domain of the AP2 core. The carboxy-terminal appendage domains of $\alpha$ and $\beta_{2}$ subunits are connected to the trunk via a flexible linker (Figure 1.5B). 


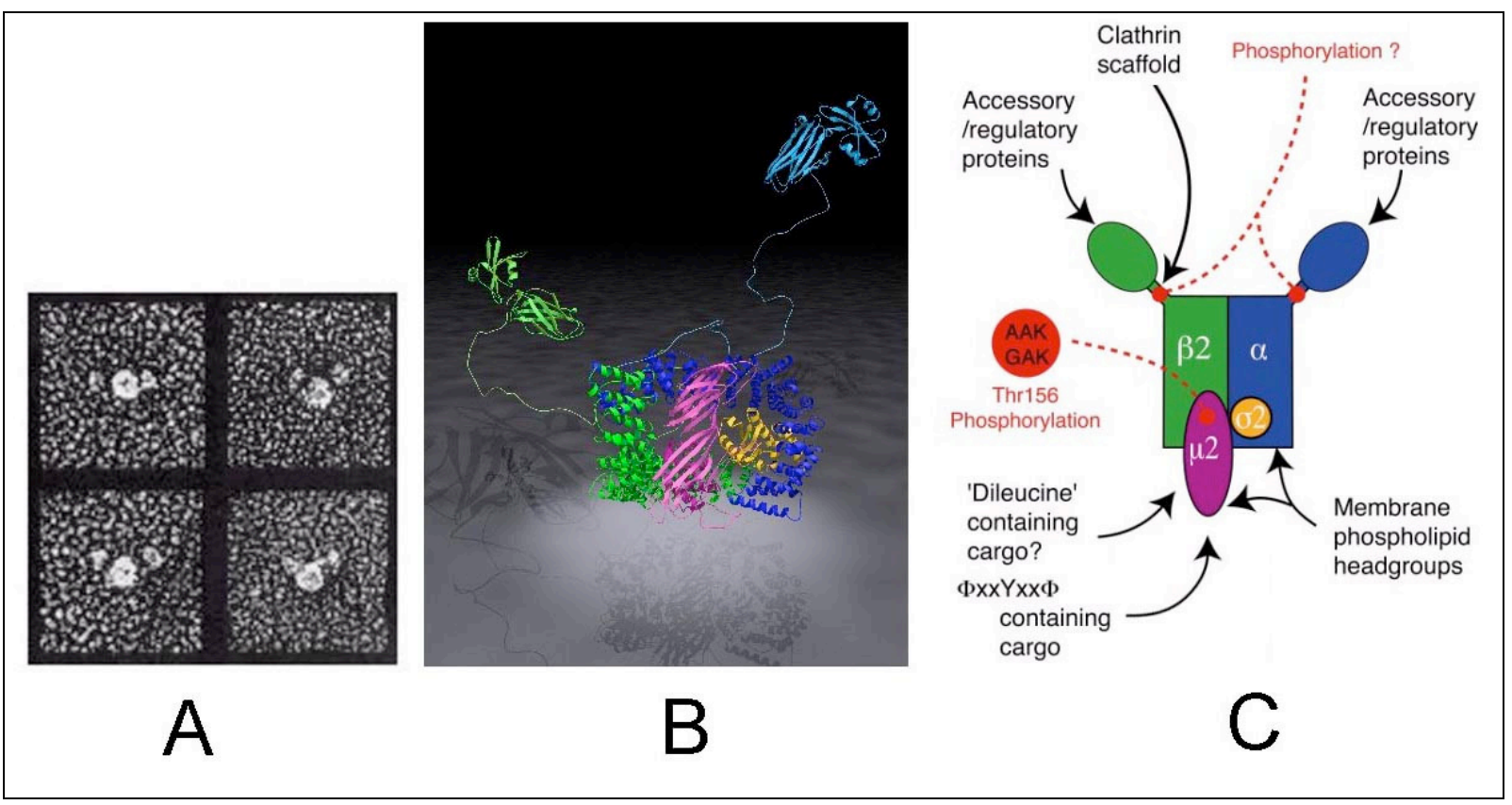

Figure 1.5 Functional and molecular organization of heterotetrameric adaptor protein AP2. (A) Deep electron micrographs of AP2 complex aid in visualization of the trunk and appendage domains separated by the flexible linker (Heuser and Keen, 1988). (B) Three dimensional reconstruction of AP2 complex with all subunits: $\alpha$ (blue), $\beta_{2}$ (green) $\mu_{2}$ (pink), $\sigma_{2}$ (orange) (C) Mapping of the protein and lipid interaction sites to the AP2 complex. (All figures were excerpted from Owen and Evans, 2002).

Interaction of AP2 with clathrin occurs by $L \phi D / E \phi D / E$ type clathrin box motifs in the $\beta_{2}$ hinge domain. $\alpha$ - and $\beta_{2}$ - appendage domains serve as a hub for recruitment of other accessory proteins to coated pits. For instance, eps15, epsin, AP180, amphiphysin, AAK 1 , synaptojanin 1 and also stonin 1 and 2 are attached to the $\alpha$-appendage domain by the virtue of short peptide motifs including DPF, DPW, FXDXF or WVXF sequences (Brett et al., 2002). The trunk domain of AP2 can be recruited to the plasma membrane by interaction of phospholipid binding sites within $\mu_{2}$ and $\alpha$ with $\mathrm{PIP}_{2}$ lipid head groups (Rohde et al., 2002; Collins et al., 2002).

The AP2- $\mu$ subunit is the main player in sorting as it recognizes plasma membrane cargo proteins by the help of tyrosine-based sorting signals within their cytosolic tails (Figure 1.5C). Another sorting signal is the dileucine motif, which has been proposed to bind to $\mu_{2-}$ or $\beta_{2}$-adaptins. Phosphorylation of the $\mu_{2}$ subunit at T156 by AAK1 enhances its binding to tyrosine-based motifs (Fingerhut et al., 2001; Ricotta et al., 2002; Conner and Schmid, 2002; Honing et al., 2005) and therefore stimulates endocytosis. Site directed mutagenesis of this threonine residue to alanine inhibits endocytosis of the transferrin receptor (Olusanya et al., 2001), and the presence of 
clathrin triskelia enhances $\mu_{2}$-phosphorylation (Jackson et al., 2003; Conner et al., 2003).

Apart from these well characterized sorting signals, some cargo proteins can also interact with AP2 through nonconventional sorting motifs (Wernick et al., 2004). Synaptotagmin 1 is also known to interact with $\mu_{2}$ by the help of a polybasic stretch of residues in its C2B domain (Haucke et al., 2000; Grass et al., 2005).

Finally, knock-down of AP2 expression by the transfection of siRNAs against $\mu_{2}$ - or $\alpha$-adaptin subunit; abolishes the internalization of some though not all membrane receptors (i.e. TfR) underlining the importance of AP2 in clathrin-mediated endocytosis (Motley et al., 2003; Hinrichsen et al., 2003).

\subsubsection{Dynamin}

A role for dynamin in endocytosis was initially proposed based on its identification as the protein encoded by the the Drosophila shibire locus. Temperature-sensitive mutations within dynamin gene causes accumulation of deeply invaginated coated pits on the plasma membrane and a following synaptic arrest (Figure 1.6) (Takei et al., 1995; Slepnev and De Camilli, 2000).

Dynamin contains a GTPase domain within its amino-terminal part and an internal GED domain which acts on the GTPase domain. It can bind to the plasma membrane via its $\mathrm{PH}$ domain and to accessory proteins bearing $\mathrm{SH} 3$ domains by virtue of its carboxy-terminal PRD domain.

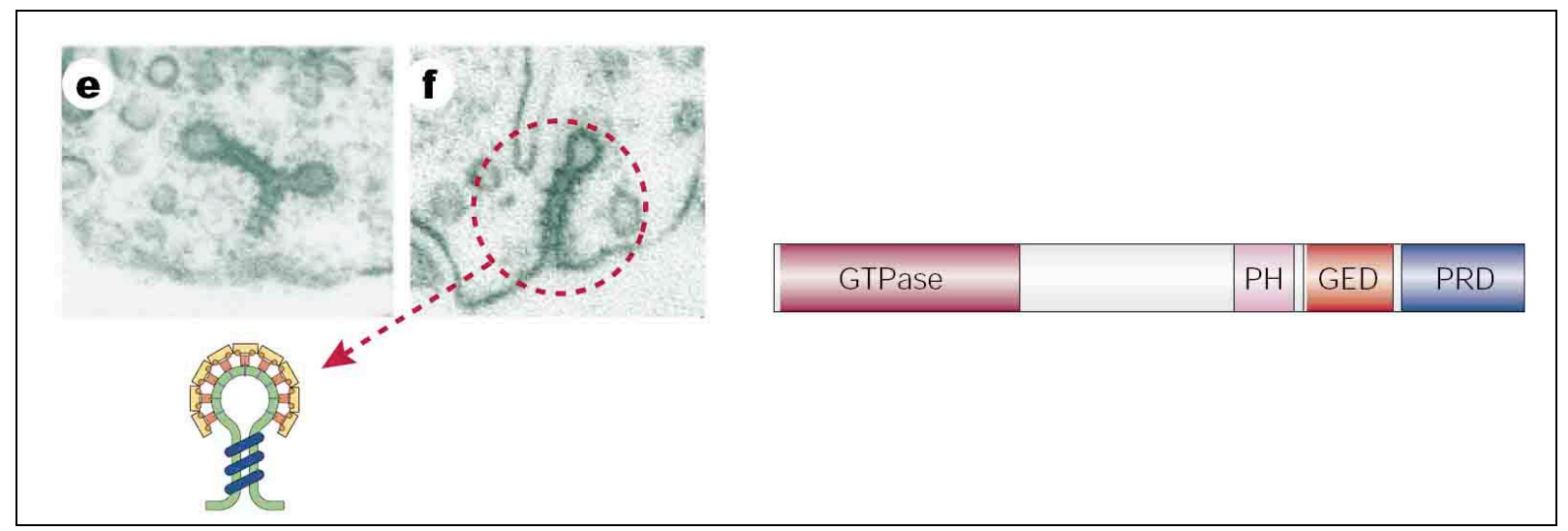

Figure 1.6 Domain organization of dynamin and effect GTP $\gamma \mathbf{S}$ on its function. In the presence of a non-hydrolysable GTP analogue (GTP $\gamma$ S), dynamin forms stacks of rings around stalks of coated pits (Takei et al., 1995). PH:pleckstrin homology domain, GED: GTPase enhancing domain, PRD: Proline rich domain.

\subsubsection{Other accessory proteins and protein-protein interaction networks}


Although AP2 is the major cargo adaptor protein in clathrin-mediated endocytosis, selective and adjustable endocytosis of numerous cargo proteins could not be accomplished by a single adaptor molecule. A growing number of endocytic accessory proteins are recruited to sites of clathrin-mediated endocytosis by their interaction with clathrin triskelia or AP2. The $\alpha$-appendage domain is the major interaction site on AP2 and it binds to endocytic proteins like eps15, epsin, AP180, numb, Dab2 and stonins (see this thesis). The associations of these proteins with AP2 are believed to be mediated by linear peptide sequences (see section 1.4.2).

In addition to direct binding to AP2 and clathrin, some accessory proteins bind to each other via protein-protein interaction domains and peptide motifs resulting in a network of protein interactions. One these networks is the EH domain-NPF motif network.

The eps 15 homology $(\mathrm{EH})$ domain is a protein recognition domain present in various cellular signaling and endocytic proteins from yeast to higher mammals. It was initially discovered as a 100 aa domain that is repeated three times in the $\mathrm{N}$-termini of the epidermal growth factor (EGF) receptor substrate eps15 and the related protein eps15R. Sequencing of the human genome has revealed $11 \mathrm{EH}$ domaincontaining proteins. Some of these proteins are also conserved in Drosophila (i.e. eps15, intersectin) or yeast (Polo et al., 2003).

Phage display assays performed to determine the recognition specificities of various $\mathrm{EH}$ domains identified an Asn-Pro-Phe (NPF) type motif that was recognized by almost all EH domains tested (Paoluzi et al., 1998). Many otherwise unrelated accessory proteins possess NPF motifs (i.e. epsin1, Numb, Hrb and stonin 2) and together with $\mathrm{EH}$ domain-containing proteins they constitute the $\mathrm{EH}$ network (Confalonieri et al., 2002).

Mammalian stonin 2 proteins harbor 2 NPF motifs in their N-terminal serine/prolinerich domain. In contrast, stonin 1 proteins do not carry NPF peptide sequences (except rodent stonin 1s which have a single NPF motif). Interactions of stonin 1 and stonin 2 with the $\mathrm{EH}$ domain containing proteins eps15, eps15R and intersectin 1 have been analyzed before (Martina et al., 2001). Mutation of either of the two NPF motifs was shown to inhibit binding of stonin 2 to eps 15 due to the low affinity of this 
interaction with $\mathrm{K}_{\mathrm{D}}$ values in the range of $500 \mu \mathrm{M}$. Consistent with this, $\mathrm{EH}$ domains and NPF motifs are usually present in multiple copies (Figure 1.7).

NMR structures of five different $\mathrm{EH}$ domains (including those from mammalian Eps15) have been determined (de Beer et al., 1998). They have a similar structural fold consisting of two helix-loop-helix motifs (also called EF hands) joined by an antiparallel $\beta$-sheet. Some of the EF hands in $\mathrm{EH}$ domains bind a single $\mathrm{Ca}^{2+}$ ion, but $\mathrm{Ca}^{2+}$ coordinating residues are not conserved in all EF hands of $\mathrm{EH}$ domains.

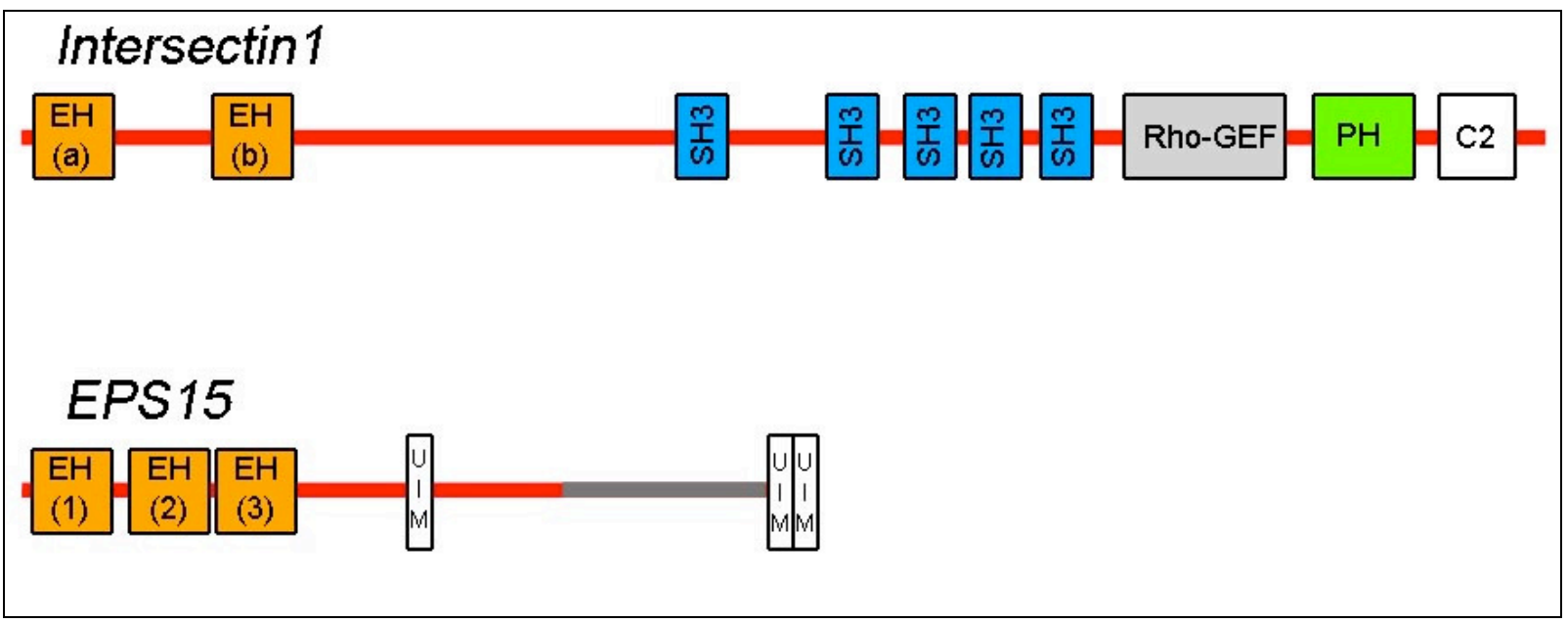

Figure 1.7 Domain and motif organization of mammalian intersectin 1 and eps15 proteins. Intersectin 1; is a $200 \mathrm{kDa}$ scaffolding protein with functions in both clathrin-mediated endocytosis and MAPK signaling. The long and short isoforms both have $2 \mathrm{EH}$ domains that interact with NPF motifs within stonin 2 and $5 \mathrm{SH} 3$ domains that bind to dynamin 1 and synaptojanin (and to stonedB in Drosophila). The long isoform in addition has a Rho-GEF domain that can act as a guanine nucleotide exchange factor for Cdc42 (Hussain et al., 2001), a pleckstrin homology (PH) domain and a putative calcium interaction domain (C2). Eps15; identified as a substrate for activated EGF-receptor has functions in both clathrin-dependent and independent endocytosis pathways. It harbors $3 \mathrm{EH}$ domains in its amino-terminal domain that bind to NPF-type motifs and 3 ubiquitin interacting motifs (UIM) that associate with monoubiquitinated proteins. It has 15 DPF motifs in its C-terminal part (gray) that are recognized by the AP2- $\alpha$-adaptin appendage domain.

\subsection{Synaptic vesicle cycle and endocytosis}

In a chemical synapse, arrival of an action potential at the presynaptic nerve terminals leads to the opening of voltage-gated $\mathrm{Ca}^{2+}$ channels which in turn cause a sharp increase in intracellular free $\mathrm{Ca}^{2+}$ concentration. This increase is transient, lasting less than $1 \mathrm{~ms}$ as $\mathrm{Ca}^{2+}$ is rapidly and effectively buffered in the presynaptic cytosol. Synaptic vesicles (SVs) sense the increase in $\mathrm{Ca}^{2+}$ concentration and exocytose their neurotransmitter contents into the presynaptic cleft (Sudhof, 2004). After exocytosis, synaptic vesicles have to be recycled locally. This process involves retrieval of SV proteins from the plasma membrane, acidification of the SV lumen, neurotransmitter refilling, docking to the presynaptic active zones and priming for a 
second round of release (Figure 1.8). Accurate and fast recycling of synaptic vesicles is essential for neurotransmission and has been implicated in synaptic plasticity (Zhang, 2003).

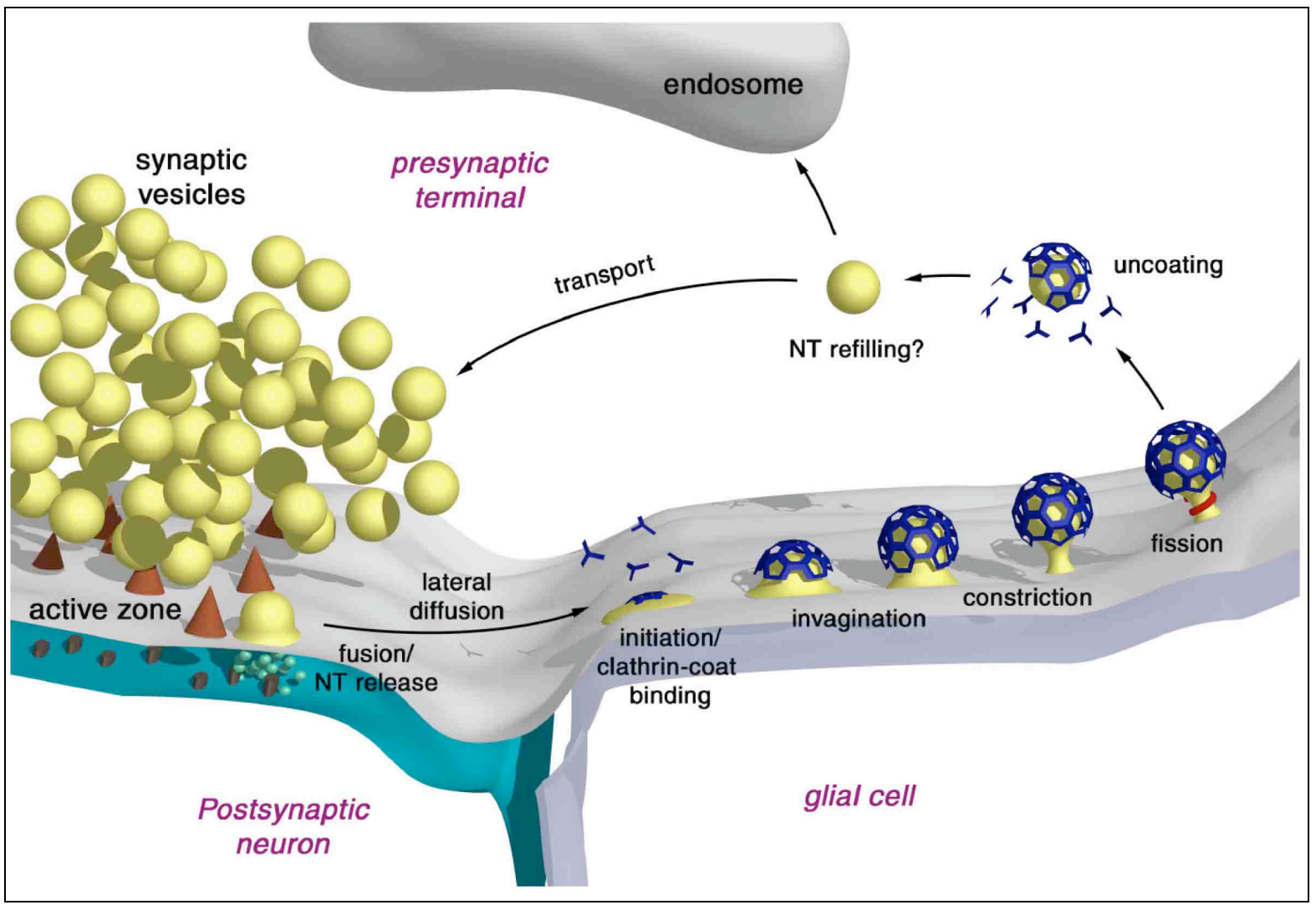

Figure 1.8 Synaptic vesicle cycle. Following fusion of synaptic vesicles at the active zones, SVs are locally recycled within the presynaptic compartment. The recycling would involve retrieval of SV proteins and lipids, acidification and refilling of the neurotransmitters, transport back to the active zones, docking and priming for a second round of release (taken from Gad, 1999).

Recycling of SV proteins and membranes would largely depend on how they have interacted with the plasma membrane during neurotransmitter release. One suggested mechanism for neurotransmitter release involves full fusion of SVs with the plasmalemma. According to this model, all SV membrane proteins would end up in the plasma membrane and have to be recycled locally by clathrin-mediated endocytosis. Since synaptic vesicles have to contain a full set of SV proteins to be competent for stimulated exocytosis (Figure 1.9A), CME thus needs to be tightly regulated to sort and retrieve proteins and lipids unique to synaptic vesicles. After shedding of the clathrin coat and neurotransmitter refilling, a functional synaptic vesicle is regenerated that returns to vesicle cluster (Figure 1.8). In accordance with this hypothesis, clathrin-coated vesicles isolated from nerve terminals contain a similar composition of proteins to that of SVs (Maycox et al., 1992; Blondeau et al., 
2004). This data suggest that CME is predominantly used for the biogenesis of SVs in brain.

Another widely accepted model suggests that a subpopulation of SVs does not collapse into the plasma membrane during secretion. Instead, they release their neurotransmitter contents by a transient opening of a fusion pore and then rapidly separate from the plasmalemma. This model of recycling has been called 'kiss and run' and does not require clathrin or other adaptor proteins for sorting and retrieval (Sudhof, 2004; Zhang, 2003; Richmond and Broadie, 2002).

Probably, both clathrin-mediated endocytosis and kiss-and-run mechanisms operate in parallel during synaptic vesicle recycling. One attractive hypothesis is that $\mathrm{CME}$ serves as a backup mechanism for kiss-and-run endocytosis, and is required to replenish synaptic vesicle pools during high frequency stimulation (Sudhof, 2004; Jarousse and Kelly, 2001b).

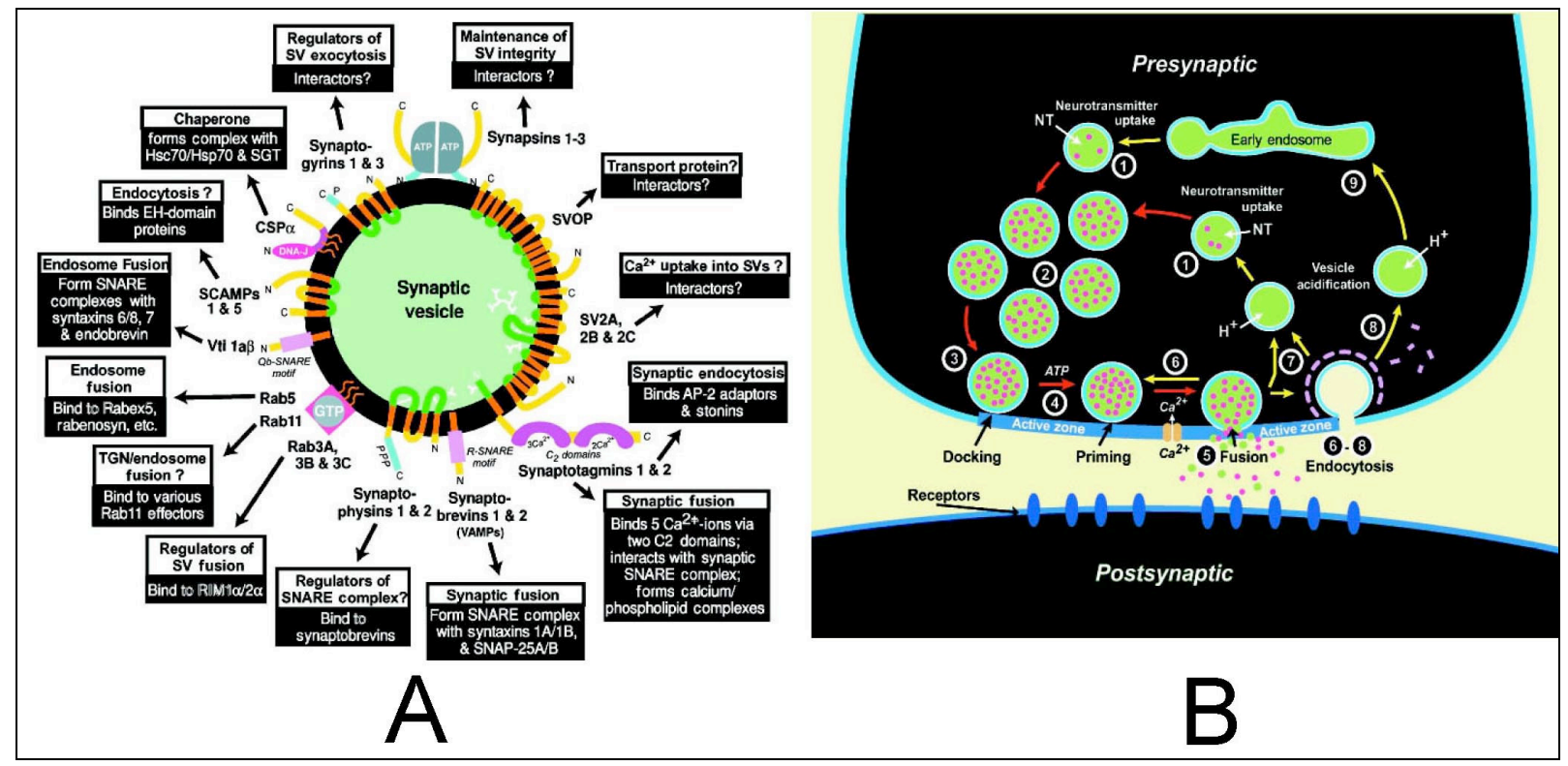

Figure 1.9 Synaptic vesicle proteins and models for synaptic vesicle cycling. (A) SVs purified from brain contain a stoichiometric ratio of proteins. Crucial SV proteins include: synaptotagmin 1\&2 $\left(\mathrm{Ca}^{2+}\right.$ sensor for exocytosis), synaptobrevins (formation of SNARE complexes during docking or fusion), neurotransmitter transporters and vacuolar ATPase (acidification of SV lumen). (B) Different proposed models fro SV recycling. According to 'kiss and run' model (7) SVs do not collapse on the plasmamembrane during secretion. 'Kiss and stay' model (6) is similar to kiss and run model, accept SVs do not leave the active zone following release and remain tethered. CME (8) which could also include an endosomal sorting event, is supposedly slower than the other models. (adapted from Sudhof, 2004)

\subsection{Synaptotagmins}

Synaptotagmins comprise a large family of proteins involved in membrane trafficking. They are conserved from nematodes to mammals. Their characteristic features 
include a short lumenal tail in the amino-terminal domain, followed by a single span transmembrane region, separated from two consecutive $\mathrm{Ca}^{2+}$ binding $\mathrm{C} 2$ domains (a proximal $\mathrm{C} 2 \mathrm{~A}$ domain and a distal $\mathrm{C} 2 \mathrm{~B}$ domain) by a variable sized linker domain (Südhof, 2002; Fukuda, 2003).

15 synaptotagmin isoforms have been identified in mammals which can be classified into several subcategories according to their similarity to each other and exon/intron boundaries (Figure 1.10)

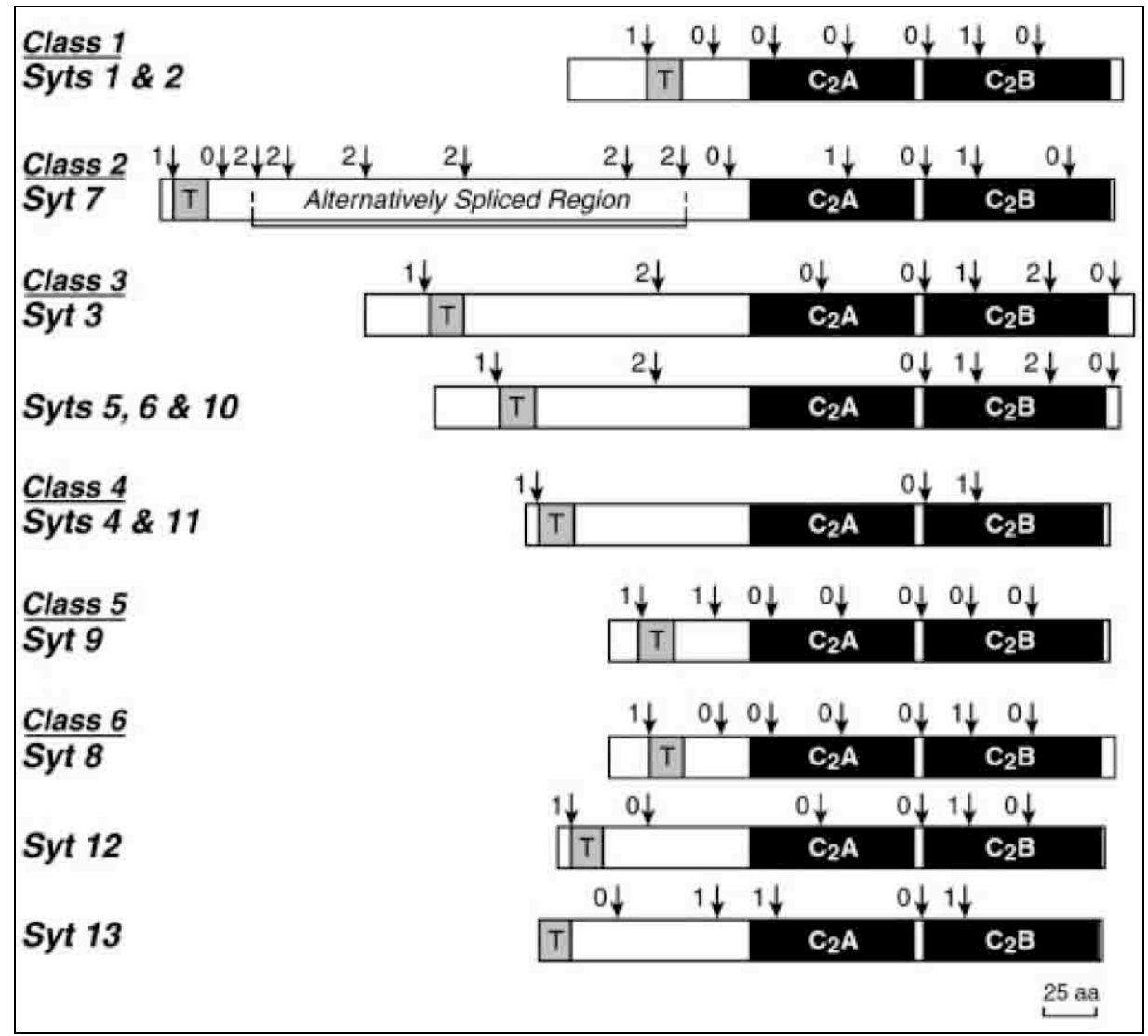

Figure 1.10 Classification and exon/intron organization of human synaptotagmin genes. The diagrams depicts single or closely related synaptotagmins identified from the human genome sequence. Exon/intron boundaries have been shown with arrows. Numbers $(0,1$ or 2$)$ near the arrows indicate the starting position of the next exon with respect to the codon sequence (excerpted from Sudhof, 2002).

Synaptotagmin 1 was initially identified in a monoclonal antibody screen for synaptic vesicle proteins. It was initially called p65 because of its apparent M.W. in SDSPAGE due to post-translational modifications. It is conserved evolutionarily from men to worms, and used as a SV marker protein in many model organisms. It is the best studied and characterized member of the synaptotagmin family.

Both synaptotagmin 1 and its evolutionarily closest homologue synaptotagmin 2 undergo three types of post-translational modifications. Synaptotagmin 1 contains $\mathrm{N}$ - 
and O-glycosylation sites in its amino-terminal (intra-lumenal) domain. Oglycosylation is promoted by the presence of synaptobrevin 2. The third kind of modification is the acylation of the cytosolic linker domain with fatty acids, a modification that seems to be conserved throughout the synaptotagmin family.

$\mathrm{C} 2 \mathrm{~A}$ and $\mathrm{C} 2 \mathrm{~B}$ domains of synaptotagmin 1 can bind to 3 and $2 \mathrm{Ca}^{2+}$ ions, respectively. However, their intrinsic affinity for $\mathrm{Ca}^{2+}$ is very low. Presence of negatively charged phospholipid head groups (i.e. phosphatidylserine, phohphatidylinositols) increases the $\mathrm{Ca}^{2+}$ affinity about 1000 fold. By contrast, neutral lipids like phosphatidylcholine or phosphatidylethanolamine have no effect. The $\mathrm{Ca}^{2+}$ binding site of synaptotagmin $1 \mathrm{C} 2$ domains is made up of 5 negatively charged Asp residues that are present on loops protruding from the $\beta$-sheets (Figure 1.11). It is believed that negatively charged lipids provide additional coordination opportunities for $\mathrm{Ca}^{2+}$ binding sites.

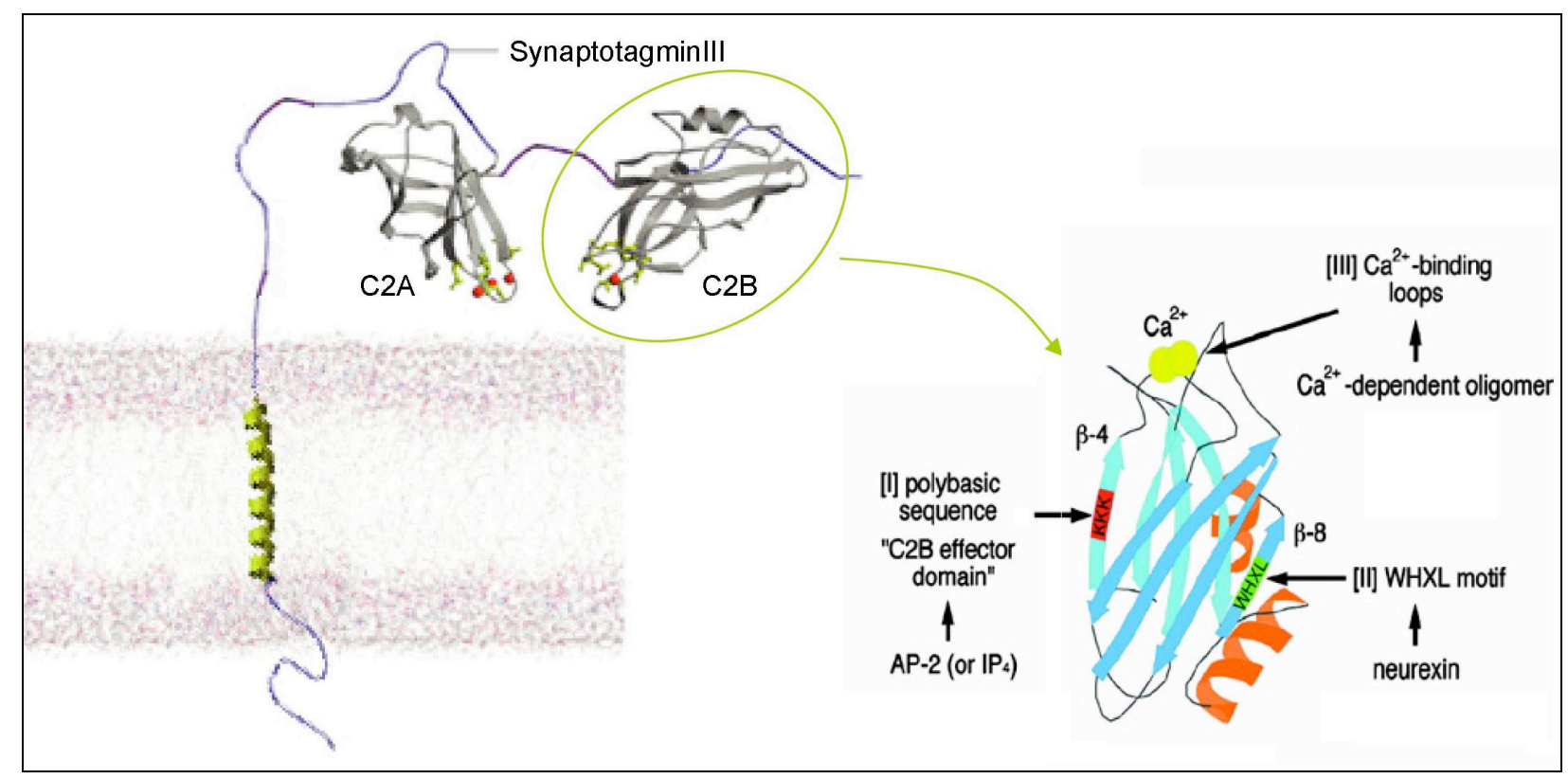

Figure 1.11 Molecular morphology of synaptotagmins and C2B domain structure. Molecular structure of synaptotagmin 3 closely resembles to that of synaptotagmin 1. Affinity of synaptotagmin 1 to negatively charged membranes is greatly facilitated in the presence of $\mathrm{Ca}^{2+}$ and vice versa. Synaptotagmin $1 \mathrm{C} 2 \mathrm{~B}$ domain binds to two $\mathrm{Ca}^{2+}$ ions. A polybasic motif (KKK) implicated in binding to the $\mu 2$ subunit of the AP2 complex and the WHXL motif which is thought to have functions in endocytosis regulation and synaptotagmin 1 docking to the plasma membrane are labeled in red and green respectively (adapted from Hui et al., 2005 and Llinas et al., 2004).

The role of synaptotagmin 1 as the major sensor for $\mathrm{Ca}^{2+}$ on $\mathrm{SVs}$ for stimulated neurotransmitter release is well established (Chapman, 2002; Schwarz, 2004). Deletion of synaptotagmin 1 in mice is lethal, abolishes fast, $\mathrm{Ca}^{2+}$ dependent synaptic 
transmission (Geppert et al., 1994). Moreover, the Asp residues that make up the $\mathrm{Ca}^{2+}$ binding site are necessary for $\mathrm{Ca}^{2+}$ stimulated exocytosis (Mackler et al., 2002).

\section{Endocytosis of synaptotagmin 1:}

In addition to its roles in $\mathrm{Ca}^{2+}$ triggered membrane fusion event at the synapses, recent studies suggest that synaptotagmin 1 is also crucial for recycling of synaptic vesicles by clathrin mediated-endocytosis (Llinas et al., 2004; Poskanzer et al., 2003; Nicholson-Tomishima et al., 2004; Schwarz, 2004).

The C2B domain of synaptotagmin 1 binds to the $\mu_{2}$ subunit of the AP2 complex in vitro and the interaction with AP2 can be further stimulated in the presence of tyrosine-based endocytic sorting motifs (Zhang et al., 1994; Haucke and De Camilli, 1999; Haucke et al., 2000). The AP2- $\mu$ interaction site within the C2B domain has been mapped to a stretch of polybasic amino acids (Figure 1.11) which were sufficient for the internalization of a tailless CD4 chimera (Haucke et al., 2000; Grass et al., 2004). Microinjection of antibodies raised against the C2B domain into squid giant synapses selectively inhibits SV recycling without influencing release, underlining the significance of the C2B domain in SV endocytosis (Llinas et al., 2004). In addition to the polybasic motif, a WHXL-type peptide motif in the carboxyterminal part of the C2B domain, which is widely conserved in synaptotagmin family members, has been shown to be required for AP2-dependent endocytosis of synaptotagmin 1 in PC12 cells (Jarousse et al., 2003).

Other relevant interaction partners of synaptotagmin 1 in SV endocytosis are Drosophila stoned proteins and their mammalian counterpart stonins (Fergestad and Broadie, 2001; Walther et al., 2001; Martina et al., 2001). Mutations in the stoned locus of Drosophila not only cause defects in SV recycling but also a partial mislocalization of synaptotagmin 1 to the plasma membrane and its degradation (Stimson et al., 1998 and 2001; Fergestad et al., 1999 and 2001).

\subsection{The Drosophila stoned mutation and its phenotypic characterization}

The Drosophila stoned mutation was identified several decades ago in a screen for stress-sensitive behavioral mutations (Grigliatti et al., 1973). FM dye uptake experiments in stoned mutants revealed a prominent decrease in the cycling synaptic vesicle pool size and defects in spatial regulation of vesicular recycling factors. Synapses of stoned mutants show a considerable delay in endocytosis of vesicular 
membranes after depolarization-induced neurotransmitter secretion (Fergestad and Broadie, 2001; Phillips et al., 2000; Stimson et al., 2001).

After the initial discovery of the temperature sensitive $s t n^{t s}$ and mechanical stress sensitive $s{ }^{C}{ }^{C}$ mutations, several other lethal stoned alleles have been identified $\left(s t n^{13-120}, s t n^{P H 1}\right.$, and $\left.s t n^{R 9-10}\right)$. All of these mutations cause death of the larvae as mature embryos as a result of the failure to hatch from the egg case. Mutant embryos are developmentally and neuro-morphologically normal but impaired in coordinated movement (Petrovich et al., 1993; Fergestad et al., 1999).

Several lines of genetic evidence suggest a function for the stoned locus in the regulation of endocytic recycling of synaptic vesicles. Temperature sensitive stoned mutants cause a synthetic lethality in combination with the shibire mutation (Petrovich et al., 1993). The shibire locus encodes dynamin, a crucial factor in CME (see section 1.4.3). In addition, combination of $s t n^{t s}$ and hypomorphic syt mutations (locus encoding Drosophila synaptotagmin) significantly decrease the viability of the adult flies (Phillips et al., 2000). A genetic interaction with a fly strain with reduced levels of DAP160 protein (Drosophila homologue of intersectin) has recently been reported (Kelly and Phillips, 2005).

The first analyis of the distribution of stoned proteins was carried out using Drosophila larvae (Fergestad et al.,1999; Stimson et al., 1998). Antibodies specific for stoned proteins were used to analyze the distribution of stoned $A$ and $B$ proteins in the nervous system. Both stoned proteins localize to synaptic sites in the central nervous system and neuromuscular junctions (NMJ) of mature embryos and developing larvae. Stoned proteins show a localization to the presynaptic site of the synaptic boutons in NMJs according to confocal microscopic imaging of double immunostained samples. The presynaptic compartment of the Drosophila NMJ can be divided into four functional subdomains: the active zone, periactive zone, membrane docked and internal synaptic vesicles and a defined network of endocytosis machinery proteins. Both stoned proteins were found to colocalize with AP2 and dynamin to the endocytotic lattice that encircles but excludes the active zone (Fergestad et al., 1999).

Defects in synaptic vesicle endocytosis as a result of the stoned mutation have been analyzed by FM1-43 dye uptake experiments at the larval NMJs (Figure 1.12D). Under depolarizing conditions, $\operatorname{stn}^{c}$ viable stoned mutants were loaded with only half 
of the amount of FM dye that had been taken up by the WT NMJs. The difference was even more significant when lethal stoned mutants were used in the same experiment (not shown). Consistently, another stoned mutant $\operatorname{stn}^{8 P 1}$ had fewer and abnormally large synaptic vesicles in larval motor terminals (Figure 1.12C).

The stoned mutation causes a reduction in the expression levels of synaptotagmin which could be detected by western blotting of protein extracts from Drosophila embryos (Fergestad et al., 1999). In addition, localization of synaptotagmin at the synaptic boutons of the larval NMJs is altered and becomes more diffuse (Figure 1.12A). Consistent with these data, measurements of the EJPs and EPCs measured in viable stoned mutants revealed that neuronal transmission is also impaired (Figure 1.12B; Stimson et al., 1998).
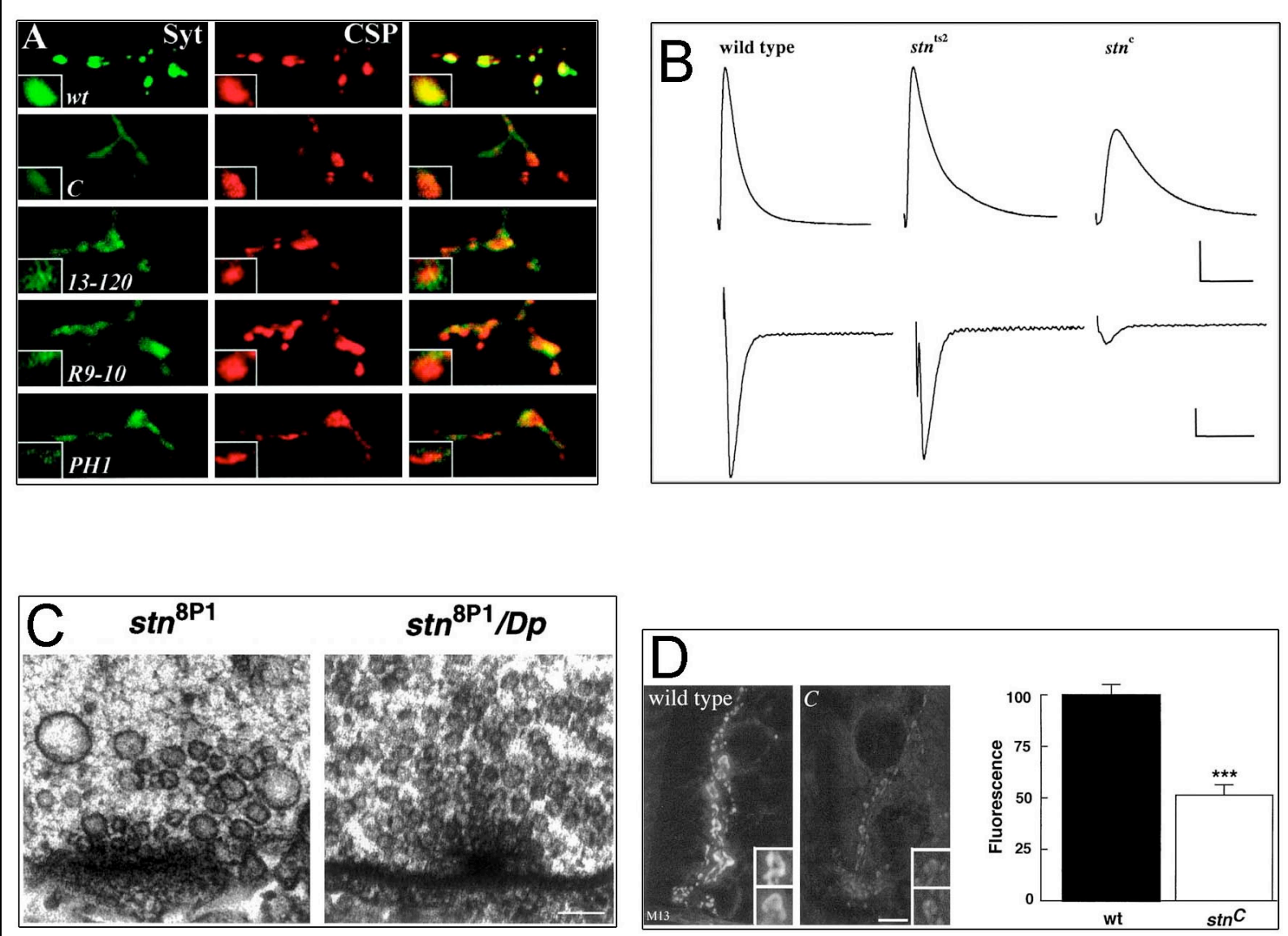

Figure 1.12 Phenotypic analyis of the Drosophila stoned mutants. (A) Synaptotagmin is reduced and mislocalized at stoned presynaptic boutons. Embryonic NMJs were double-labeled with antibodies against SV protein Syt ( green) and the SV-associated protein CSP (red). The Syt-staining pattern in wild-type embryos shows distinct punctate expression in the boutons that colocalizes with CSP expression. Mutant synapses have reduced and mislocalized Syt expression but maintain punctate CSP expression. Syt expression in mutants appears dispersed throughout the presynaptic terminal including innervating axons. (B) The $s \operatorname{sn}^{t s 2}$ and $\operatorname{stn}^{\mathrm{c}}$ mutations affect both spontaneous (not shown) and evoked synaptic activity at the larval NMJ. Representative EJPs (top traces) and EJCs (bottom traces) recorded from wild-type, $s t n{ }^{t s 2}$, and $\operatorname{stn}^{\mathrm{C}} \mathrm{NMJs}$ at $1.5 \mathrm{mM} \mathrm{Ca} 21$ (C) The $\operatorname{stn}^{8 P 1}$ mutation alters 
synaptic vesicle number and size. Shown are electron micrographs (EMs) through motor terminals on larval body wall muscles 6 and 7 . High magnification views of active zones show that $\operatorname{stn}^{8 P 1}$ boutons have abnormally large vesicles rarely observed in controls $\left(s t n^{8 P 1 / D p}\right)$. Scale bar, $100 \mathrm{~nm}$. In $s t n^{8 P 1}$ mutants the synaptic vesicle density is reduced significantly, whereas synaptic vesicle size is increased significantly. (D) Viable stoned mutant $\operatorname{stn}^{c}$ show reduced FM1-43 dye uptake at the Drosophila NMJ. Terminals were loaded with dye by incubating preparations in $90 \mathrm{mM} \mathrm{K} 1$ saline for 5 $\mathrm{min}$ in the presence of $10 \mathrm{mM} \mathrm{FM1-43.} \mathrm{Dye} \mathrm{uptake} \mathrm{in} \operatorname{stn}^{C}$ boutons is significantly reduced $(50 \%)$ compared with that of controls. ( Excerpted from Fergestad et al., 1999; Stimson et al., 1998; Stimson et al., 2001; Fergestad and Broadie, 2001; Copyright Society for Neuroscience)

Most of the recorded defects in stoned mutation-carrying animals arise from the degradation of synaptotagmin. Overexpression of synaptotagmin in a stoned lethal background rescues embryonic lethality and restores endocytic recycling to normal levels (Fergestad and Broadie, 2001).

Quite recently, it has been shown that the phenotype seen in Drosophila stoned mutants is exclusively due to the absence of the stoned $B$ protein, the downstream translation product of the bicistronic stoned mRNA. Transgenic expression of stoned $B$ but not stoned $A$ in an otherwise stoned lethal background restores synaptic properties and viability (Estes et al., 2003).

\subsection{Sequence analysis of stoned and stonin genes}

The Drosophila stoned locus encodes a bicistronic transcipt which, upon translation gives rise to two gene products, the stoned $A$ and stoned $B$ proteins.

Stoned $A$ is a protein with no homology to other known proteins. It harbors 5 DPF motifs (Figure 1.13) that might be recognized by the platform subdomain of the $\alpha$ adaptin-appendage domain of AP2- $\alpha$. The only known orthologues of stoned A are found in the genomes of other arthropods; mosquito (Anopheles gambiae) and honey bee (Apis mellifera). Like their Drosophila counterpart, mosquito stoned A contains 6 DPF motifs and bee stoned A has 7 DPF motifs. Remarkably, bee stoned A also harbors a WVGFDEE sequence that (as a part of this thesis) we and others have shown to bind to the sandwich subdomain of the AP2- $\alpha$-appendage. This suggests that WVXF-type AP2 interacting motifs had already evolved in arthropods.

The second open reading frame within the stoned mRNA encodes for the stoned $B$ protein. Stoned B harbors a region of homology with the carboxy terminal, cargo binding domain of the $\mu 2$ subunit of the AP2 complex. This region in stoned $B$ and its orthologues is known as the $\mu$-homology domain $(\mu \mathrm{HD})$ and consists of about 300 


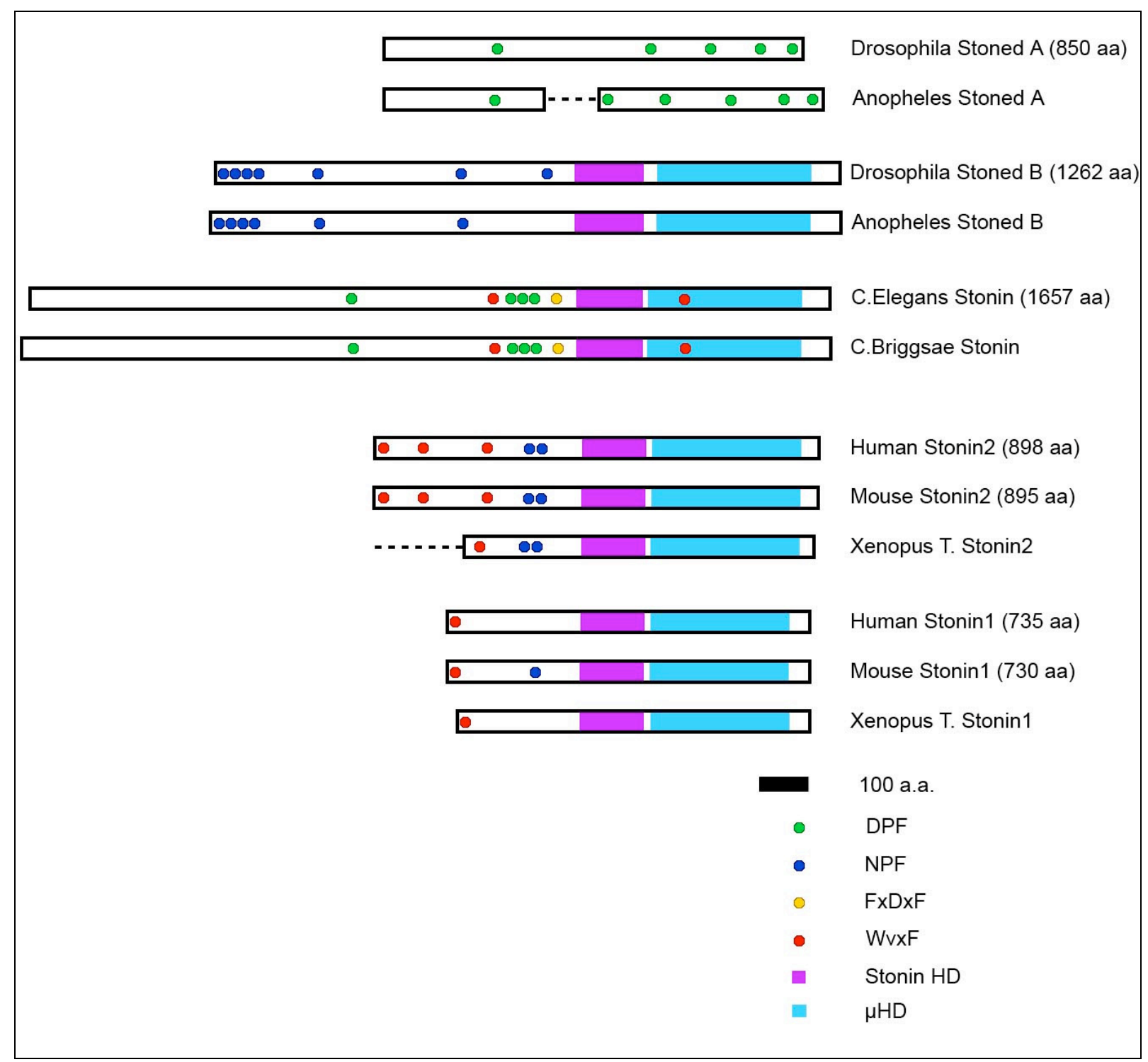

Figure 1.13 Orthologues of stoned genes from selected species. Orthologues of stoned proteins from different species were identified by BLAST searches of the genomic DNA sequences deposited at the NCBI or Ensembl data bases using tblastn or tblastx algorithms. Dashed lines show regions of the proteins that could not be retrieved because of the incompleteness of the genomic sequences. Numbers in parantheses show length of the amino acid sequences that are known for certain.

amino acid residues at the carboxy-terminal domain of stoned B proteins (see Figure 1.13 and 1.14). Upstream of the $\mu \mathrm{HD}$, there is another conserved stretch of 130 amino acids that is only found in stoned B orthologues. This domain has been termed the stonin homology domain (StoHD) to indicate that it is exclusively seen in stonins. The amino terminal part of the protein is rich in serine/proline residues and might be unstructured based on secondary structure predictions. There are seven NPF motifs in the amino-terminal part of Drosophila stoned B. These motifs are recognized by EH domains of other endocytic accessory proteins such as eps15. Eps15 localizes to clathrin-coated pits via interactions with AP2 and clathrin and thereby may recruit 
stoned $B$ to the endocytic machinery. This modular organization of domains and motifs in Drosophila stoned B is conserved in all of its orthologues.

Nematode stonins (also known as adaptin-related protein, apt-10 or unc-41 in C. elegans) harbor four DPF motifs and one FXDXF motif that is also known to bind to the platform subdomain of the AP2- $\alpha$-appendage. Even though the WVXF-type motifs found in these stonins have the basic elements (tryptophane at first position, phenylalanine at fourth position and a negatively charged residue at one of the $5^{\text {th }}, 6^{\text {th }}$ or $7^{\text {th }}$ positions), it is not known whether they actually bind to AP2. In contrast to Drosophila stoned $B$, nematode stonins encompass sequence motifs that would enable them bind to AP2 directly. The presence of several DPF motifs in the very large amino terminal domain of nematode stonins suggests that they may share common ancestry with the bicistronic stoned transcipts of the arthropods.

Vertebrates and higher animal genomes contain two stonin genes: stonin 1 and stonin 2. Stonin 1 sequences are less conserved between different species compared to stonin 2 suggesting that the latter might have a more crucial physiological role. The overall homology between stonin 1 and 2 is restricted to the $\mathrm{C}$-terminal stoHD and $\mu \mathrm{HD}$. The amino-terminal domain sequences are unrelated except for being rich in serines and prolines, and harboring AP2 interaction motifs. Stonin 1 and stonin 2 contain one and three copies of WVXF motif respectively. In addition, stonin 2 contains two NPF motifs that were shown to bind to intersectin 1 , eps15 and eps15R. Rat and mouse stonin 1 proteins each harbor one copy of the NPF motif. Whether or not this single NPF sequence is capable of associating with $\mathrm{EH}$ domain proteins remains uncertain.

Most likely stonin 1 and stonin 2 genes have arisen by a locus duplication event during the early evolution of higher animals. Close syntheny of stonin 1 and stonin 2 loci supports this hypothesis (Table 1.1). Furthermore, the last 3 coding exons of both genes are of similar length, located at similar distances with respect to each other, and contain paralogous sequences (compare Figure 1.15 and Figure 2.5). 


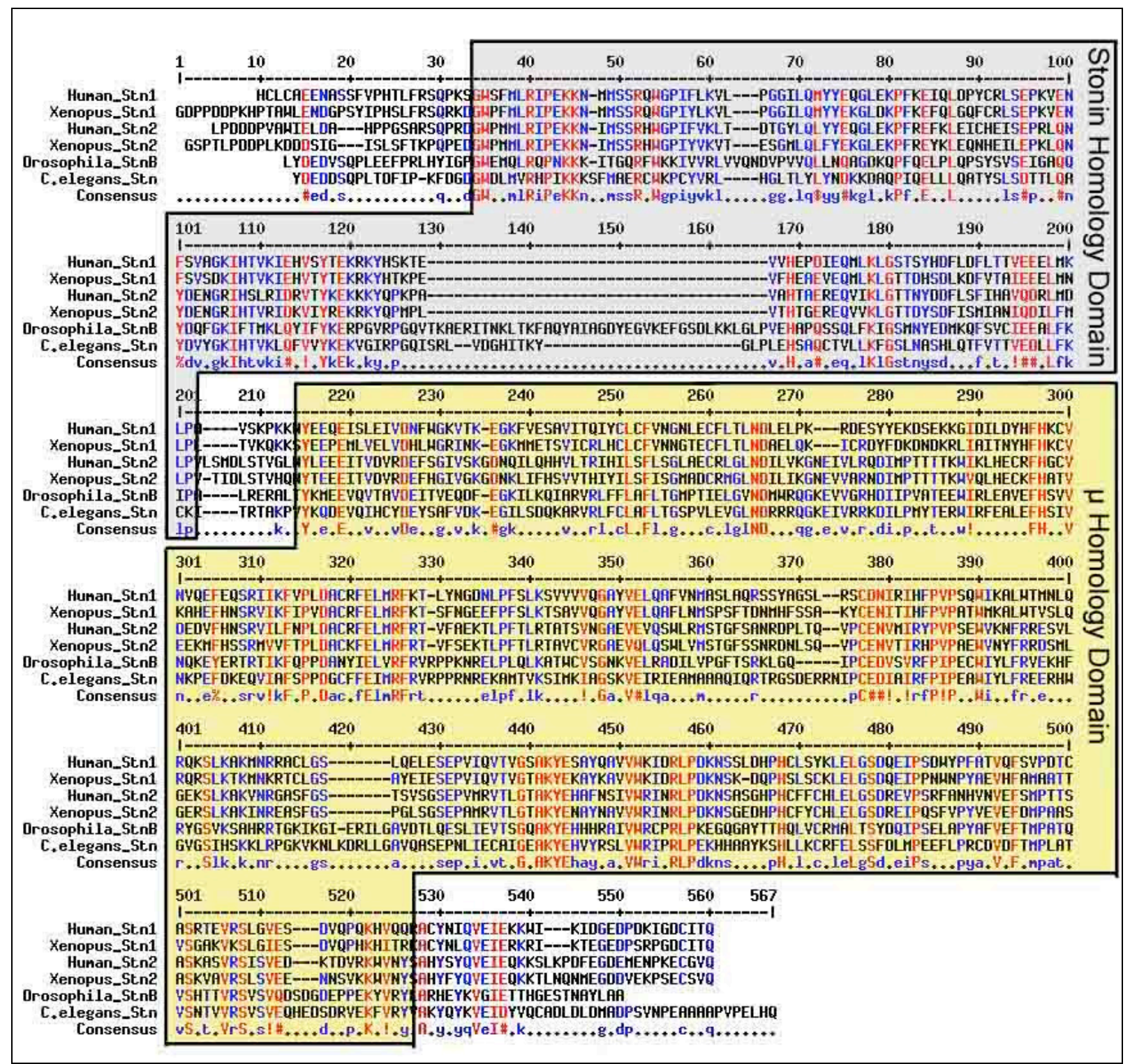

Figure 1.14 Multiple sequence alignment of stonins. Partial sequences including stonin and $\mu$ homology domains from the indicated stonins were aligned using the MultAlin interface at http://prodes.toulouse.inra.fr/multalin/multalin.html according to parameters defined by (Corpet, 1988). Red colored residues indicate $\geq 90 \%$ consensus and blue indicate $\geq 50 \%$ consensus.

\begin{tabular}{|c|c|c|c|c|c|c|}
\hline \multicolumn{7}{|c|}{ Stonin1 Locus Chromosome 2 Region: $48210000.00 . .49316000 .00$} \\
\hline start & stop & Symbol & $\mathrm{O}$ & Cyto & Description & Genebank \\
\hline 48719414 & 48737696 & SBLF & + & $2 \mathrm{p} 16.3$ & stoned B-like factor & NM_006873 \\
\hline 48756599 & 48871938 & ALF & + & 2p16.3 & TFIIA-alpha/beta-like factor & NM_006872 \\
\hline 48825618 & 48894468 & -HCGR & - & 2p21 & $\begin{array}{l}\text { luteinizing hormone/choriogonadotropin } \\
\text { receptor }\end{array}$ & NM_000233 \\
\hline \multicolumn{7}{|c|}{ Stonin2 Locus Chromosome 14 Region: $80230581.00 . .81510761 .00$} \\
\hline start & stop & Symbol & $\mathrm{O}$ & Cyto & Description & Genebank \\
\hline 80491679 & 80680525 & ISHR & + & $14 q 31$ & thyroid stimulating hormone receptor & NM_000369 \\
\hline 80716147 & 80757328 & GTF2A1 & - & $14 q 31.1$ & general transcription factor IIA, 1, 19/37kDa & NM_201595 \\
\hline 80806662 & 80934680 & STN2 & - & $14 q 31.1$ & stonin 2 & NM_033104 \\
\hline
\end{tabular}

Table 1.1 Close syntheny of human stonin 1 and stonin 2 gene loci. Human stonin 1 and stonin 2 locus gene maps (Homo sapiens genome, build 35.1) were obtained from the NCBI web site at 
http://www.ncbi.nlm.nih.gov/mapview. Genes denoted by bold letters are homologous to each other proven by sequence alignment.

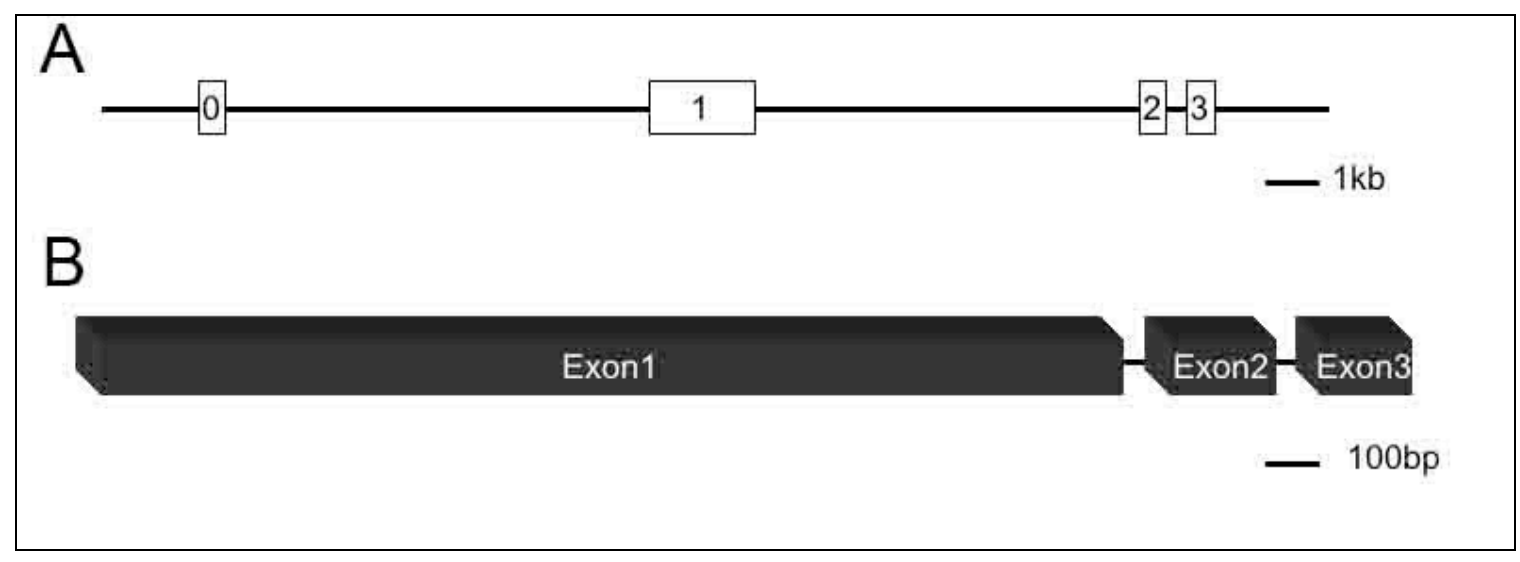

Figure 1.15 Genomic organization of the mouse stonin 1 locus. (A) The mouse stonin 1 gene (coding exons) is spread over a $10.5 \mathrm{~kb}$ genomic locus on chromosome 17 (between nucleotides 87249242 and 87259424 ). It consists of 3 protein coding exons and at least one additional exon coding for the 5 ' untranslated region of the stonin 1 mRNA that is separated by an 8 kb sequence from the remainder of the gene. Distances between the depicted exons reflect the original ratios. (B) Schematic diagram depicting the relative lengths of stonin 1 exon sequences. Exon1 (1-1916), Exon2 (1917-2118), Exon3 (2119-2193).

An alternative and rare transcript for stonin 1 has been isolated from human cDNA libraries (Upadhyaya et al., 1999; Chen et al., 2001). This transcript has been named SALF (Stoned B/TFIIA $\alpha / \beta$-like factor) and consists of a fusion between stonin 1 and TFIIA $\alpha / \beta$-like factor transcipts. It was identified from an incomplete human placental cDNA sequence (I.M.A.G.E. Consortium Clone ID 259637) that contained pieces of stonin 1 and ALF (TFIIA $\alpha / \beta$-like factor). Several lines of evidence suggest that the SALF transcript is rare and without any physiological significance but may instead arise from erroneous transcription or splicing. First, our analysis of mammalian EST sequences at the $\mathrm{NCBI}$ database using the SALF peptide sequence as a query identified 142 positive matches. However, only one of these ESTs had a sequence aligning with both stonin 1 and ALF coding parts of the the SALF transcript and this particular EST was the same clone used in identification of the SALF transcript (Upadhyaya et al., 1999).

Second, northern blot analysis shows that the SALF transcript is detected only in testis as a $1.8 \mathrm{~kb}$ mRNA when a probe specific for the ALF sequence is used. ALF has a 478 a.a. long sequence which corresponds well to the molecular weight of the detected mRNA. However, a probe specific for the stonin 1 sequence detected a 6.5 $\mathrm{kb}$ mRNA that was present at the highest levels in heart, placenta, kidney, prostate, and uterus and at lower levels in other tissues but not in testis (Upadhyaya et al., 
1999; Martina et al., 2001). Since the ALF specific probe does not detect any mRNAs from these tissues, it is safe to say that the $1.8 \mathrm{~kb}$ and $6.5 \mathrm{~kb}$ mRNAs detected by the two probes are entirely different.

It is possible to find several stonin 2 variants in gene and protein databases with varying sequence lengths and contents. However, there is only one physiological transcript known for stonin 2 which is translated into a protein with 898 amino acids in humans and 895 in mice. The 702 aa long human stonin 2 variant cloned by (Martina et al., 2001) lacks the first 3 and the last coding exon. The 905 aa variant cloned by us (Walther et al., 2001), although present as mRNA does not contain the last exon, resulting in an alternative carboxy-terminal sequence shared with the 702 aa variant (the last 30 residues are different). Apparently the intronic region between $5^{\text {th }}$ and $6^{\text {th }}$ coding exons is sometimes incorrectly spliced, resulting in an alternative carboxyterminal end.

One interesting feature of the mammalian stonin 2 protein (also arthropod stoned B's and nematode stonins) is its highly acidic amino-terminal sequence. The serine/proline rich amino-terminal domain is also enriched in aspartate and glutamate residues and this results in a very low isoelectric point of 4.1 . The carboxy-terminal domain made up of StoHD and $\mu \mathrm{HD}$ has a p.I. near neutral $\mathrm{pH}$. In contrast to stonin 2 , stonin 1 proteins are also neutral or only slightly acidic. The highly acidic aminoterminal sequence causes abnormal migration of stonin 2 in SDS-PAGE (130kDa apparent M.W. instead of $100 \mathrm{kDa}$ as predicted from its a.a. sequence).

The carboxy-terminal $\mu \mathrm{HD}$ s of stonin 1 and stonin 2 show a low, but significant homology to $\mu 1 \mathrm{~A}, \mu 1 \mathrm{~B}$ and $\mu 2$ subunits of AP1 and AP2 complexes but not to $\mu 3 \mathrm{~A}$, $\mu 3 B$ or $\mu 4$ subunits of AP3 and AP4. Interestingly, clustal analysis of all $\mu$ subunits and $\mu \mathrm{Hd}$ s from stonin 1 and stonin 2 show that stonin $\mu \mathrm{HD}$ s are more closely related to $\mu 1$ and $\mu 2$ than to $\mu 3$ and $\mu 4$ (Figure 1.16).

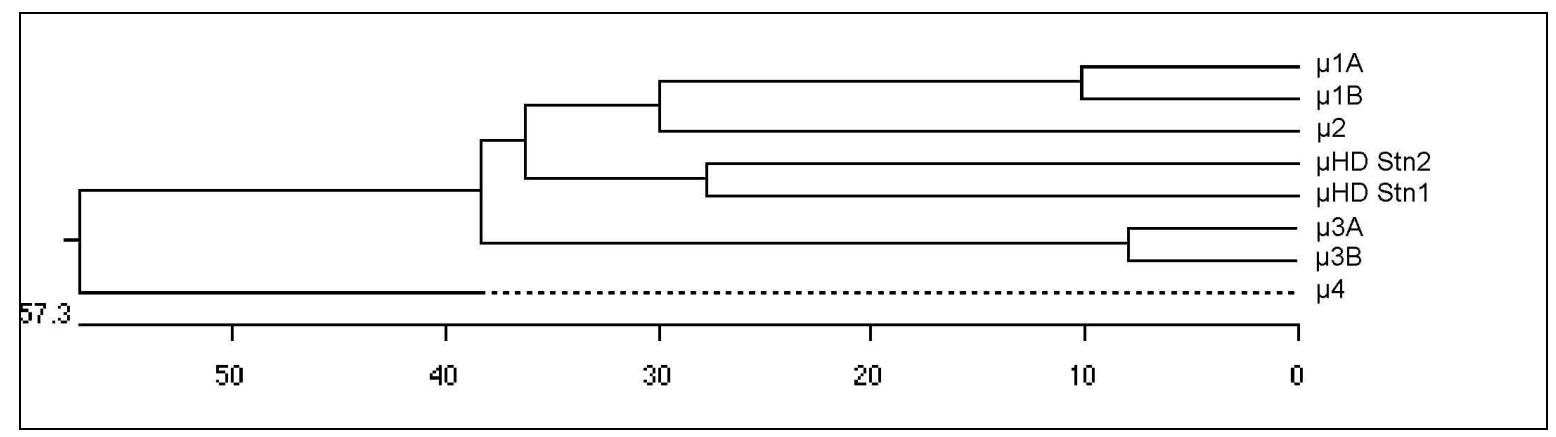


Figure 1.16 Phylogenetic analysis of $\mu$ subunits and $\mu$ HDs. Peptide sequences from mouse $\mu$ subunits and human stonin $\mu \mathrm{HDs}$ were aligned using the clustal method of the Megalign software. Phylogenetic tree suggests that $\mu \mathrm{HDs}$ and $\mu 1$ and $\mu 2$ subunits have a common ancestor.

The $\mu 2$ subunit of the AP2 complex can be divided into two subdomains: An aminoterminal $\beta$-binding domain (roughly first 150 residues) which makes close interactions with the $\beta$ subunit. This subdomain is required for stabilizing the overall architecture of the tetrameric complex. The carboxy-terminal subdomain is composed of about 20 parallel $\beta$-sheets, and its overall architecture closely resembles a 'banana'. This domain (C- $\mu 2)$ harbors the binding pocket for $Y X X \Phi$-type endocytic sorting motifs derived from the cytosolic tails of membrane cargo proteins. C- $\mu 2$ also interacts with membranes via a $\mathrm{PIP}_{2}$ binding site (Rohde et al.,2002), and binds to the cytoplasmic domains of synaptotagmins 1 and 2 (Martina et al.,2001).

Blast 2 alignment of peptide sequences of stonin $\mu \mathrm{HDs}$ with $\mu 2$ shows a homology that is restricted to the cargo binding domain $(C-\mu 2)$. The last 30 residues of the stonin $1 \mu \mathrm{HD}$ domain do not align with $\mu 2$ perhaps providing an explanation why stonin 1 does not associate with synaptotagmin 1 .

Comparison of stonin2- $\mu \mathrm{HD}$ alignment with the known crystal structure of $\mu 2$ shows that the stretches of residues that do not align (represented with dashed lines in Figure 1.17) correspond to loops between the $\beta$-sheets. $\beta$-sheet sequences by contrast appear to be highly conserved between both proteins. Therefore, one would predict that the stonin $2 \mu \mathrm{HD}$ may adapt a similar overall structure to $\mathrm{C}-\mu 2$. 
Score $=60.8$ bits $(146)$, Expect $=2 e-07$

Identities $=65 / 273(23 \%)$, Positives $=122 / 273(43 \%)$, Gaps $=51 / 273(18 \%)$

Stonin1 : 410 YEEQE ISLE IVDNFWGKVTKEGKFVESAVITQIYCLCFVNGNLECFLTLND-LELPKRDE 468 $\mathrm{Y} \quad \mathrm{E}+\mathrm{L}++++++++\mathrm{G}+++\mathrm{V}++\quad+++\mathrm{G}$ EC $+\mathrm{ND}++\mathrm{K}++$ 12 : 168 YRRNELFLDVLESVNLLMSPQGQVLSAHVSGRVVMKSYLSGMPECKFGMNDKIVIEKQGK 227

Stonin1 : 469 SYYEKDSE--KKGIDILDYHFHKCVNVQEFEQSRI IKFVPLDACRFELMRFKTLYNGDNL 526 $++\mathrm{S}+\mathrm{K}+\mathrm{I}$ I D $\mathrm{FH}+\mathrm{CV}++\mathrm{F}+$ R I F+P D FELMR++T L 12 : 228 GTADETSKSGKQSIAIDDCTFHQCVRLSKFDSERSISFIPPDG-EFELMRYRTT-KDIIL 285

Stonin1 : 527 PFSLKSVVVVQG-AYVELQAFVNMASLAQRSSYAGSLRSCDNIRIHFPVPSQWIKALWTM 585 $\mathrm{PF}++\mathrm{V} \quad \mathrm{G}+\mathrm{E}+++\quad+\mathrm{S}++\mathrm{SL}+\mathrm{I}+\mathrm{P} \mathrm{P} \quad \mathrm{L} \mathrm{T}$ 12 : 286 PFRVIPLVREVGRTKLEVKVVI-------KSNFKPSLLA-QKIEVRIPTP------LNTS 331

Stonin1 : 586 NLQRQKSLKAKMNRRACLGSLQELESEPVIQVTVGSAKYESAYQAVVWKIDRLPDKNSSL 645 $+Q+$ G AKY +++ A+VWKI R+ S $12 \quad: 332 \mathrm{GVQ}$

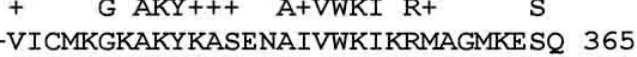

Stonin1 : 646 DHPHCLSYKLELGSDQEIPSDWYPFATVQFSVP 678

12 : 366 ----ISAEIELLPTNDKKKWARPPISMNFEVP 393

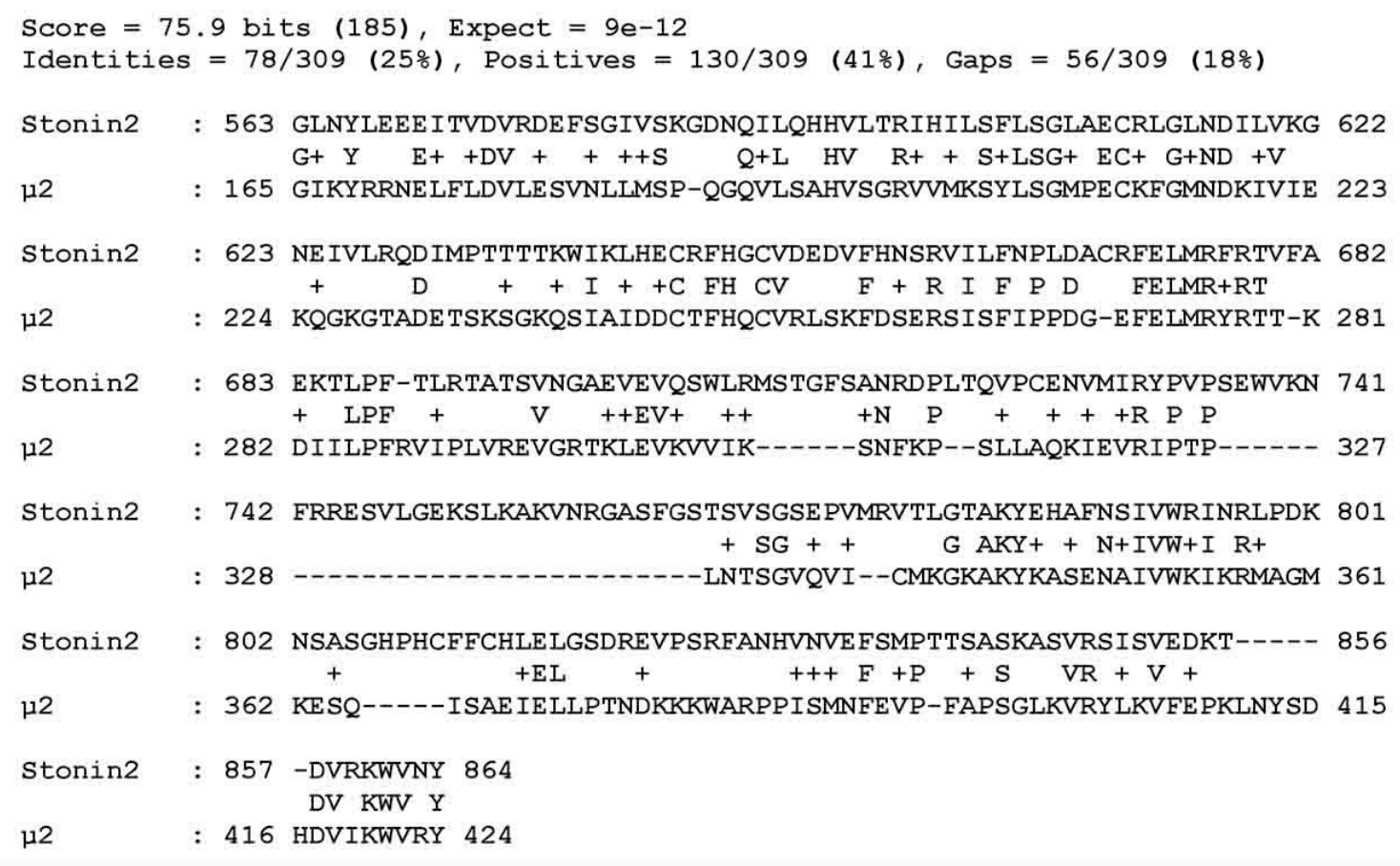

Figure 1.17 Alignment of human stonin 1 and stonin 2 with mouse $\mu 2$. Protein sequence alignments of human stonin1 and stonin2 with mouse $\mu 2$ were performed at the NCBI web interface http://www.ncbi.nlm.nih.gov/blast/bl2seq using BLOSUM62 matrix. 


\subsection{Aims of the study}

Clathrin mediated endocytosis is an important mechanism for recycling of presynaptic vesicle membrane proteins and lipids. Phenotypic analysis of Drosophila stoned mutants have indicated that stoned proteins, in particular stoned B, play an important role in synaptic vesicle endocytosis by a mechanism that involves interactions with the synaptic vesicle membrane protein synaptotagmin 1 . There are two mammalian orthologues of Drosophila stoned B; stonin 1 and stonin 2. Mammalian stonin 2 has been shown to interact with the endocytic proteins AP2, eps15 and intersectin 1 as well as with synaptotagmins 1 and 2 . Our studies were thus based on the hypothesis that stonin 2 may function in clathrin/AP2 mediated endocytic recycling of synaptic vesicles a mechanism that has been conserved through evolution.

Specifically, the aim of this thesis was to investigate the roles of mammalian stonins 1 and 2 in clathrin-mediated endocytosis and synaptic vesicle recycling. As an initial approach we aimed at further characterizing the interactions of stonins with the endocytic proteins AP2, eps15, and intersectin 1 by biochemical means. In order to shed light on the physiological role of stonin 2 in clathrin-mediated membrane traffic, we generated and initially characterized stonin 2 knock-out mice and analyzed the cell biological functions of stonin 2 in reconstituted cellular systems including neuroendocrine PC12 cells. Based on our results we propose that stonins may have a broader role as endocytic accessory proteins in neurons as well as other cell types including astroglial cells. 


\section{MATERIALS AND METHODS}

\subsection{MATERIALS}

\subsubsection{Antibodies}

\subsubsection{Primary antibodies}

\begin{tabular}{|c|c|c|c|c|}
\hline Name & Applications, dilution & Source & Species & $\begin{array}{l}\text { Clone, batch, other } \\
\text { description }\end{array}$ \\
\hline Amphiphysin 1 & IB(1:2000) IP & P. De Camilli & rabbit & CD5, SH3 domain \\
\hline $\mathrm{AP} 1-\gamma$ & $\mathrm{IB}(1: 100)$ & BD Biosciences & mouse & \\
\hline AP2 & IF(1:1000) IP & P. De Camilli & mouse & AP6 \\
\hline AP2- $\alpha$ & IF(1:1000) & BD Biosciences & mouse & \\
\hline AP2- $\beta 1 / \beta 2$ & $\mathrm{IB}(1: 1000)$ & BD Biosciences & mouse & \\
\hline AP2- $\mu 2$ & $\mathrm{IB}(1: 250)$ & BD Biosciences & mouse & \\
\hline CD25 & $\mathrm{IF}(1: 500) \mathrm{Al}$ & R.A. Kroczek, RKI. & mouse & Anti Tac antigen \\
\hline $\mathrm{CHC}$ & IF(1:1000) & Affinity bioreagents & mouse & $\mathrm{X} 22$ \\
\hline Cytochrome c & $\operatorname{IF}(1: 200)$ & BD Biosciences & mouse & Clone 6H2.B4 \\
\hline EEA1 & $\operatorname{IF}(1: 50)$ & BD Biosciences & mouse & \\
\hline EHD3 & $\mathrm{IB}(1: 5000)$ & Markus Plomann & rabbit & \\
\hline Eps15 & $\mathrm{IB}(1: 200)$ & BD Biosciences & mouse & \\
\hline FLAG tag & IF $(1: 400)$ IB $(1: 1000)$ IP AI & Sigma & mouse & clone M2 \\
\hline GFAP & $\operatorname{IF}(1: 100)$ & K.A. Nave & mouse & \\
\hline HA tag & $\operatorname{IF}(1: 400) \operatorname{IB}(1: 1000)$ IP $\mathrm{AI}$ & Babco & mouse & \\
\hline $\mathrm{Hsc} 70$ & $\mathrm{IB}(1: 1000)$ & Affinity bioreagents & mouse & \\
\hline Intersectin & $\mathrm{IB}(1: 200)$ & P.McPherson & rabbit & \\
\hline Myc tag & IF(1:400) IP AI & Sigma & mouse & Clone 9E10 \\
\hline Stonin1 & $\operatorname{IF}(1: 50) \operatorname{IB}(?) \operatorname{IP}(?)$ & Self made & rabbit & \\
\hline Stonin2-2424.5 & $\operatorname{IF}(1: 200) \operatorname{IB}(1: 1000)$ IP & Self made & rabbit & Against stn2NT \\
\hline Stonin2-54 & $\operatorname{IF}(1: 20) \operatorname{IB}(1: 100)$ & Self made & rabbit & Anti stn2 peptide \\
\hline Synaptophysin & $\operatorname{IF}(1: 1000) \operatorname{IB}(1: 1000)$ & Synaptic Systems & rabbit & \\
\hline Synaptotagmin 1 & IF(1:1000) IB(1:2000) IP & Synaptic Systems & mouse & Clone 41.1 \\
\hline Synaptotagmin1 & $\mathrm{IF}(1: 1000) \mathrm{AI}$ & R. Jahn & rabbit & 89.07 lumenal \\
\hline Synaptotagmin1 & $\mathrm{IF}(1: 200) \mathrm{Al}$ & Synaptic Systems & mouse & 604.4 lumenal \\
\hline Synaptotagmin1,2 & $\operatorname{IF}(1: 100)$ & Synaptic Systems & rabbit & \\
\hline T7 tag & $\operatorname{IF}(1: 200)$ & Novagen & mouse & \\
\hline Transferrin Rec. & $\operatorname{IF}(1: 400)$ & Zymed & mouse & \\
\hline
\end{tabular}

Table 2.1 Primary antibodies 


\subsubsection{Secondary antibodies}

$\begin{array}{lllll}\text { Name } & \begin{array}{l}\text { Applications, } \\ \text { dilution }\end{array} & \text { Source } & \text { Species } & \begin{array}{l}\text { Clone, batch, other } \\ \text { description }\end{array} \\ \text { Alexa }^{488} \text { Goat } \alpha \text { Mouse } & \mathrm{IF}(1: 200) & \text { Molecular Probes } & \text { goat } & \\ \text { Alexa }^{488} \text { Goat } \alpha \text { Rabbit } & \mathrm{IF}(1: 200) & \text { Molecular Probes } & \text { goat } & \\ \text { Alexa }^{594} \text { Goat } \alpha \text { Mouse } & \mathrm{IF}(1: 200) & \text { Molecular Probes } & \text { goat } & \\ \text { Alexa }{ }^{594} \text { Goat } \alpha \text { Rabbit } & \mathrm{IF}(1: 200) & \text { Molecular Probes } & \text { goat } & \\ \text { Cy5 Goat } \alpha \text { Rabbit } & \mathrm{IF}(1: 100) & \text { Jackson Immunores } & \text { goat } & \\ \text { Goat } \alpha \text { Mouse-HRP } & \mathrm{IB}(1: 5000) & \text { Jackson Immunores } & \text { goat } & \text { HRP conjugated } \\ \text { Goat } \alpha \text { Rabbit-HRP } & \mathrm{IB}(1: 10000) & \text { Jackson Immunores } & \text { goat } & \text { HRP conjugated } \\ \text { Goat } \alpha \text { Mouse-AP } & \mathrm{IB}(1: 2000) & \text { Jackson Immunores } & \text { goat } & \text { AP conjugated } \\ \text { Goat } \alpha \text { Rabbit-AP } & \mathrm{IB}(1: 2000) & \text { Jackson Immunores } & \text { goat } & \text { AP conjugated } \\ \text { Goat } \alpha \text { Mouse } & \mathrm{Blocking}(1: 10) & \text { Jackson Immunores } & \text { goat } & \text { serum, unlabeled } \\ \text { Goat } \alpha \text { Rabbit } & \mathrm{Blocking}(1: 10) & \text { Jackson Immunores } & \text { goat } & \text { serum, unlabeled } \\ \text { Rabbit } \alpha \text { Mouse } & \mathrm{IB}(1: 500) & \text { Jackson Immunores } & \text { rabbit } & \text { unlabeled }\end{array}$

Table 2.2 Secondary antibodies

\subsubsection{Oligonucleotide primers and siRNAs}

\subsubsection{Primers}

\begin{tabular}{|c|c|c|}
\hline & primer name & sequence \\
\hline 1 & $\operatorname{Pr}-1$ (mm_stonin2) & gggggcactgaagagcacttcccagga \\
\hline 2 & Pr-2(mm_stonin2) & ggtggtggtgggcatgatgtcctgcc \\
\hline 3 & Pr-3(mm_stonin2) & ggcaggacatcatgcccaccaccacc \\
\hline 4 & Pr-4(mm_stonin2) & ggagcctgtaagtgtgcagaataattgacccacttc \\
\hline 5 & $\operatorname{Pr}-5\left(m m \_s t o n i n 2\right)$ & atgacgaccttggaccatgtcattgccaccca \\
\hline 6 & Pr-6(mm_stonin2) & gctggtgtcctcagaatgcgtggtcca \\
\hline 7 & Pr-7(mm_stonin2) & ccgggaagtgccttccagatttgccaac \\
\hline 8 & Pr-8(mm_stonin2) & tggaccacgcattctgaggacaccagc \\
\hline 9 & Pr-9(mm_stonin2) & atggaagccatcagttggcaggcaggcagt \\
\hline 10 & Pr-10(mm_stonin2) & atgtcctctaggcactggggaccaatctac \\
\hline 11 & $\operatorname{Pr}-11\left(m m \_s t o n i n 2\right)$ & tcagtagattggtccccagtgcctagaggacat \\
\hline 12 & Pr-12(mm_stonin2) & cccgcgagggattatttcattaaagtcagagca \\
\hline 13 & Pr-13(mm_stonin2) & ggtgttatcccactgggtgc \\
\hline 14 & Pr-14(mm_stonin2) & acacctgcctctgccttcca \\
\hline 15 & Pr-15(mm_stonin2) & caggagaaggcatcggatcca \\
\hline 16 & $\operatorname{Pr}-16\left(m m \_s t o n i n 2\right)$ & gagcagccacagcaggtgt \\
\hline 17 & Pr-17(mm_stonin2) & gggaagccctctccaggctac \\
\hline 18 & Pr-18(mm_stonin2) & ggggggcagtctccct \\
\hline 19 & Pr-19(mm_stonin2) & gggaagagatgtgtaacagtca \\
\hline 20 & Pr-20(mm_stonin2) & ggtcctccagggcggetcctca \\
\hline
\end{tabular}

purpose of use

cDNA cloning and sequencing

cDNA cloning and sequencing

cDNA cloning and sequencing

cDNA cloning and sequencing

cDNA cloning and sequencing

cDNA cloning and sequencing

cDNA cloning and sequencing

cDNA cloning and sequencing

cDNA cloning and sequencing

cDNA cloning and sequencing

cDNA cloning and sequencing

cDNA cloning and sequencing

genomic DNA sequencing

genomic DNA sequencing

genomic DNA sequencing

genomic DNA sequencing

genomic DNA sequencing

genomic DNA sequencing

genomic DNA sequencing

genomic DNA sequencing 
Pr-21(mm_stonin2)

ggggtggtccetgtcactgtaccccaca

gatc cccggg geccctcacctgcctca

23 Pr-23(mm_stonin2)

$\operatorname{Pr}-24\left(m m \_s t o n i n 2\right)$

25 5'BamHI(mm_stonin2)

3'HinD3(mm_stonin2)

48 Pr-2+Notl(mm_stonin1)

49 ESC_Screen(Up1)

50 ESC_Screen(Up2)

51 ESC_Screen(Low1)

52 ESC_Screen(Low2)

53 pUC18_Forward

54 pUC18_Reverse

55 Neo_Fwd1

56 Neo_Rev1 gatc ctcgag ctaggacagcactggtaaatcca

gatc gcggccgc tcactgtacccca

gatc ggatcc atgacgaccttggaccatgt

gatc aagctt ctatataagaagcatctgctggaag

gact gatatc gaggagcaggagaggcg

gact gatatc cggtcccagcctcgtga

gccagaaacactagtgggcacagggtaacgg

ccgttaccctgtgcccactagtgtttctggc

gact tctaga tcaagttgtgggcatgctgaac

gact tctaga tcaataattgacccacttcctg

gact tctaga tcactcaatttccacctggtagc

gact tctaga tcaatctccttcaaagtccggcttc

atgtattctacaaacccgggcagctgg

ttattgagttacacagccaccagttttatctgc

gatc gaattc atgtattctacaaacccgggc

gatc gcggccgc ttattgagttacacagccacc

cgagccggaagtggagcagatgctg

gggcttggcagtagccggcagc

ccaggaaccttcagaggcagaagtccctga

cgtcccgtttctggagctcacggtcgt

gatc ctcgag ctactttctgcttgccaggga

gatc gaattc ccgttctcaccaaaggaca

gatc gaattc agctacgaggaacaagaaa

gatc gaattc cctgcttttcaatcttctc

gatc ctcgag ctacaccctgagtctccagaga

gatc gatcgc

ggccgcgggcttggcagtagccggagc

ccacgcagtcggatccttctcaa

gcgcatgcctgctctcgacca

ctgcagggtcgetcggtgttc

ctgggcggggtttgctcgaca

cgccagggtttcccagtcacgac

gtgagcggataacaatttcacacagga

ggcgcggtcccaggtccac

cttcgcccaatagcagccagtcc
cDNA sequencing

cDNA cloning (StoninHD), genotyping

cDNA cloning (StoninHD), genotyping

cDNA cloning ( $\mathrm{C}$ terminus)

cDNA cloning ( $\mathrm{N}$ terminus)

cDNA cloning (alternative $\mathrm{C}$ terminus)

cDNA cloning, 2335 truncation

cDNA cloning, 2420 truncation

cDNA cloning, $\mathrm{C}$ terminal deletion

cDNA cloning, $\mathrm{C}$ terminal deletion

cDNA cloning, $\mathrm{C}$ terminal truncation

cDNA cloning, $C$ terminal truncation

cDNA cloning, $\mathrm{C}$ terminal truncation

cDNA cloning, $\mathrm{C}$ terminal truncation

cDNA cloning

cDNA cloning

cDNA cloning

cDNA cloning

cDNA cloning, sequencing

cDNA cloning, sequencing

cDNA cloning, sequencing

cDNA cloning, sequencing

cDNA cloning

cDNA cloning

cDNA cloning

cDNA cloning

cDNA cloning

cDNA cloning

ESC colony screening

ESC colony screening

ESC colony screening

ESC colony screening

sequencing

sequencing

genotyping

genotyping

\section{Table 2.3 Oligonucleotide primers}

\subsubsection{SiRNAs}

For silencing of $\mu 2$ expression, an siRNA directed against human $\mu 2$ sequence was used (Motley et al., 2003). This $\alpha-\mu 2$ siRNA was equally effective against simian $\mu 2$. 
Target sequence : AAGUGGAUGCCUUUCGGGUCA

\subsubsection{Enzymes and proteins}

If not otherwise mentioned within the text, the enzymes and proteins used in this study were purchased from New England Biolabs, Sigma, Gibco-BRL, Stratagene, MBI Fermenta, Roche and Invitrogen.

\subsubsection{Chemicals}

All chemicals used in this study were analytical grade purity and they were purchased from Merck, Roth, Sigma and Gibco.

\subsubsection{Radioactive materials}

$\left[\alpha-{ }^{32} \mathrm{P}\right] \mathrm{dCTP}$ (labeling of DNA probes for southern hybridization) and $\left[{ }^{125} \mathrm{I}\right.$ protein $\mathrm{A}$ (used for quantitative immunoblots) were purchased from Amersham.

\subsubsection{Plasmid vectors}

\subsubsection{Standard vectors}

The following plasmid vectors were obtained from the mentioned companies. Detailed information (i.e. sequence, MCS, promotor) about these vectors is readily available from the company web sites, NCBI vector database or elsewhere in the internet.

$\begin{array}{lll}\text { pGEX class vectors } & : & \text { Amersham } \\ \text { pET class vectors } & : & \text { Novagen } \\ \text { pEGFP class vectors } & : & \text { BD Biosciences } \\ \text { pcDNA3 } & : & \text { Invitrogen } \\ \text { pcDNA3.1/N5-His-TOPO } & : & \text { Invitrogen } \\ \text { pBlueScriptll KS } & : & \text { Stratagene } \\ \text { pUC18 } & : & \text { Invitrogen }\end{array}$

\subsubsection{Custom design vectors}

pcHA : A mammalian cell expression vector with a pcDNA3 backbone and a pBlueScriptll SK(+) MCS (restriction sites between BamHI and Xhol were replaced). It introduces an HA-tag to the N-termini of cloned cDNAs. It has a strong CMV promotor that drives the expression of the cloned genes. 

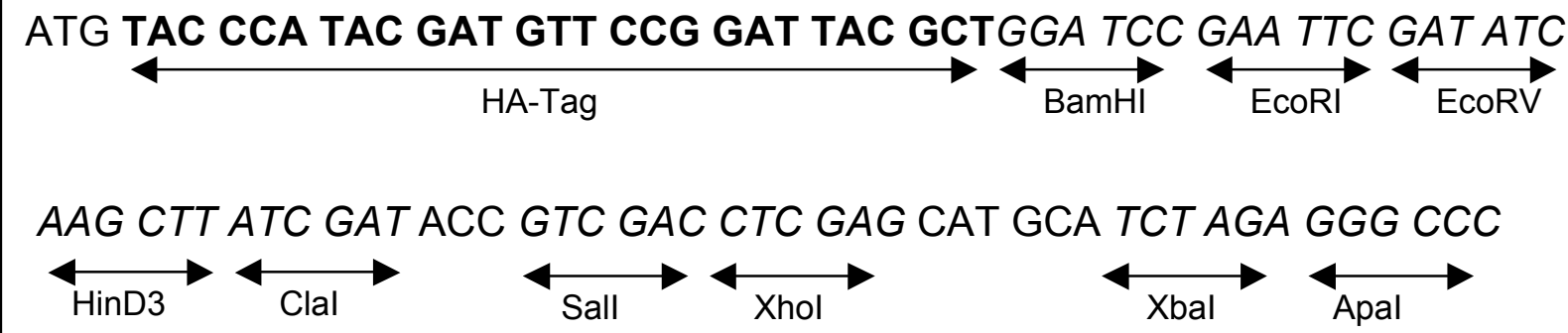

pcFLAG: Similar to pcHA2 in design, however contains a FLAG-tag instead of an HA-tag.
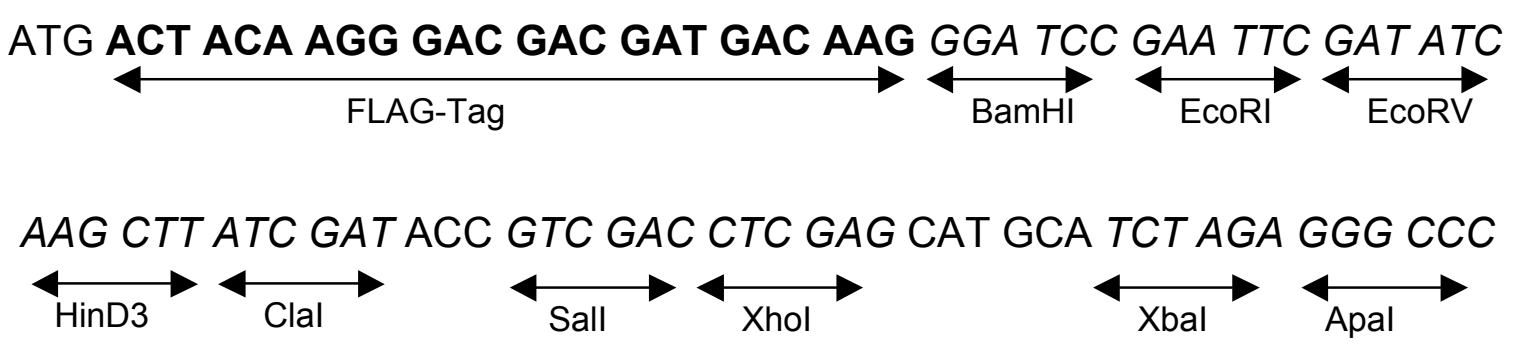

\subsubsection{Kits}

Kit name

Wizard Plus SV minipreps

E.Z.N.A. Cycle pure kit

Profection Kit

Qiagen Plasmid midi kit

QiaexII Gel Extraction kit

Rediprimell random prime labeling system

ECL Western blotting detection reagents and analysis system
Purpose of use

Supplier

mini scale plasmid preps for sequencing and Promega mammalian cell transfection

purification of DNA from PCR or restriction Peqlab digests

mammalian cell transfection with calcium Promega phosphate

To obtain increased purity and quantity of plasmid DNA

Extraction of DNA fragments from agarose Qiagen gels for cloning

Radioactive labeling of southern

Amersham hybridization probes

detection of immunoblots by

Amersham chemiluminiscence system

Qiagen
pcDNA3.1/N5-His-TOPO TA high efficiency TA cloning of PCR fragments Invitrogen cloning kit

BCA protein assay kit measurement of protein concentration

Pierce

Table 2.4 Kits 


\subsubsection{Commonly used software and web interfaces}

Lasergene sequence analysis software

Adobe Photoshop CS 8.0.1

Adobe 6.0 Professional

CN3D 4.1 software for analysis of biological macromolecule structures

Microsoft Office Applications

Slidebook 4.0.8 Digital Microscopy Software

National Center for Biotechnology Information web site

Ensembl Genome Browser

Protein Data Bank

ExPASy Proteomics Server
DNASTAR Inc.

Adobe Systems Inc.

Available at

http://www.ncbi.nlm.nih.gov/Structure/CN3D/cn3 d.shtml

Microsoft Corp.

Intelligent Imaging Innovations

web address: http://www.ncbi.nlm.nih.gov

web address: http://www.ensembl.org

web address: http://www.rcsb.org/pdb

web address: http://au.expasy.org

Table 2.5 Software and web-interfaces

\subsubsection{Microscopes and special instruments}

Zeiss Axiovert 200M Digital Research Microscopy System

Hund Wilovert Standard Inverted Microscope

Olympus SZ61 stereo microscope with KL1500LCD cold light source

Confocal Laser Scanning Microscope II

Mini Protean II Electrophoresis system

FastBlot B44

Gene Pulser II Electroporation System

Optima TLX Ultracentrifuge

Cyclone Autoradiography System
Carl Zeiss AG

Helmut Hund Gmbh

Olympus

Carl Zeiss AG

Bio-Rad

Whatman Biometra

Bio-Rad

Beckman Coulter

Packard

Table 2.6 Microscopes and special instruments

\subsubsection{Bacterial strains and mammalian cell lines}

\subsubsection{Bacterial strains}

BL21(DE3) E. Coli cells [F- ompT hsdS $\left.\left(\mathrm{r}_{\mathrm{B}}^{-} \mathrm{m}_{\mathrm{B}}{ }^{-}\right) \mathrm{gal} \mathrm{dcm}\right]$ Invitrogen

Top10 E. Coli cells [F-mcrA $\Delta$ (mrr-hsdRMS-mcrBC) $\phi 80$ lacZ $\Delta$ M15 $\Delta$ lacX74 Invitrogen recA1 ara $\Delta 139 \Delta$ (ara-leu)7697 galU galK rpsL $\left(\mathrm{Str}^{\mathrm{R}}\right)$ endA1 nupG] 


\subsubsection{Mammalian cell lines}

HEK293 Human embryonal kidney cells

COS7 African green monkey kidney cells

U343 Human astrocytoma cells

N1E-115 Mouse neuroblastoma cells

INS-1E Rat insulinoma cells with $\beta$-cell phenotype

PC12 Rat adrenal pheochromocytoma line

ES14-1 Mouse 129SV/J embryonic stem cell line

\subsubsection{Commonly used buffers, solutions and media}

LB medium, 1It

2XYT medium, 1It

LB plates, 1lt

Fixative, 100ml

10X TBE electrophoresis buffer, 1lt

TE buffer

SNET buffer

10X TBS

10X PBS

High salt PBS

GSDB(Goat serum dilution buffer)

GSDB-light

SDS-PAGE running buffer

SDS-PAGE 4X Stacking gel buffer

SDS-PAGE 4X Separating gel
$10 \mathrm{~g}$ tryptone, $5 \mathrm{~g}$ yeast extract, $5 \mathrm{gNaCl}, 1 \mathrm{ml} 1 \mathrm{~N}$ $\mathrm{NaOH}$ (autoclaved)

$16 \mathrm{~g}$ tryptone, $10 \mathrm{~g}$ yeast extract, $5 \mathrm{~g} \mathrm{NaCl}, 1 \mathrm{ml}$ $1 \mathrm{~N} \mathrm{NaOH}$ (autoclaved)

$10 \mathrm{~g}$ tryptone, $5 \mathrm{~g}$ yeast extract, $5 \mathrm{~g} \mathrm{NaCl}, 15 \mathrm{~g}$ agar (autoclave in a flask, cool down to 40$45^{\circ} \mathrm{C}$, add antibiotics and pour into petri dishes)

Dissolve $4 \mathrm{~g}$ paraformaldehyde in PBS by stirring at $60^{\circ} \mathrm{C}$. Cool down and filter. Store aliquots at $-20^{\circ} \mathrm{C}$ until use.

$108 \mathrm{~g}$ tris base, $55 \mathrm{~g}$ boric acid, $20 \mathrm{mM}$ EDTA $\mathrm{pH}$ 8.0

10mM TrisCl pH 8.0, 1mM EDTA pH 8.0

20mM TrisCl pH 8.0, 5mM EDTA pH 8.0, $400 \mathrm{mM} \mathrm{NaCl}, 1 \%$ SDS

$200 \mathrm{mM}$ TrisCl pH 7.4, 1.4M NaCl

1.37M NaCl, 27mM KCl, 43mM Na $2 \mathrm{HPO}_{4}$, $14 \mathrm{mM} \mathrm{NaH}_{2} \mathrm{PO} 4$

$20 \mathrm{mM} \mathrm{NaPO}_{4} \mathrm{pH}_{7.4,500 \mathrm{mM} \mathrm{NaCl}}$

$25 \%$ Goat serum, 0.3\%(v/v) Triton X-100 in high salt PBS

Same as GSDB but $150 \mathrm{mM} \mathrm{NaCl}$

246mM Tris, 1.92M Glycin, 10\%SDS

$0.4 \%$ SDS, 0.5M Tris- $\mathrm{Cl} \mathrm{pH} 6.8$

$0.4 \%$ SDS, $1.5 \mathrm{M}$ Tris- $\mathrm{Cl} \mathrm{pH} 8.8$ 
buffer

TBS-T

Blotto

Antibody solution

Ponceau staining solution Immunoblot transfer buffer 6X SDS-PAGE sample buffer 6X DNA loading buffer

Coomassie blue SDS-PAGE staining solution

SDS-PAGE destaining solution 20X SSC for southern blots

Neutralization buffer for southern blots

Denaturation solution for southern blots

Mitomycin $\mathrm{C}$ stock solution $1 \mathrm{mg} / \mathrm{ml}$ Table 2.7 Buffers and media
$0.1 \%(v / v)$ Tween 20 in TBS $4 \%(w / v)$ non fat milk powder in TBS-T $2 \%(\mathrm{w} / \mathrm{v})$ Analytical grade BSA and $0.02 \% \mathrm{NaN}_{3}$ in TBS-T

$0.3 \%(\mathrm{w} / \mathrm{v})$ Ponceau $\mathrm{S}$ in $1 \%$ acetic acid $80 \%$ SDS-PAGE running buffer, $20 \% \mathrm{MetOH}$

$0.05 \%$ bromophenol blue, $0.05 \%$ xylene cyanol, $30 \%$ glycerol

$0.1 \%$ (w/v) Coomassie 250G, 10\% acetic acid, $25 \%$ methanol in $\mathrm{ddH}_{2} \mathrm{O}$

$10 \%$ acetic acid, $25 \%$ methanol in $\mathrm{ddH}_{2} \mathrm{O}$

$3 \mathrm{M} \mathrm{NaCl}, 0.3 \mathrm{M} \mathrm{Na}_{3}$ Citrate

1.5M NaCl, $1 \mathrm{M}$ Tris/ $\mathrm{HCl} \mathrm{pH} 7.2$

$1.5 \mathrm{M} \mathrm{NaCl}, 0.5 \mathrm{M} \mathrm{NaOH}$

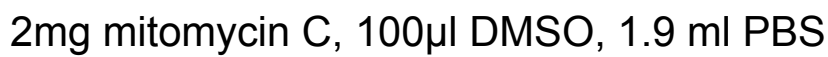

\subsection{METHODS}

\subsubsection{Molecular biology methods}

For all molecular biology experiments, protocols derived from Sambrook and Russell (2001), Molecular Cloning, were used.

\subsubsection{Polymerase Chain Reaction}

For cloning applications which require high fidelity PCR, Vent polymerase (NEB) was used. Taq polymerase (from Fermentas or Genaxxon) was used for all other applications. All primers were purchased from MWG-Biotech and they ranged in size of 20-40 nucleotides. Annealing temperatures $5^{\circ} \mathrm{C}$ below the $T_{m}$ of the primers have been used as a starting point and optimized when necessary. Template DNA was denatured at $94^{\circ} \mathrm{C}$ for 2-10 minutes prior to PCR cycles. Extension times ranged from 30 seconds to 4-5 minutes depending on the length of the DNA fragment to be amplified and the enzyme used. For instance, when amplifying with Taq polymerase, 1 minute extension time is required per 1000 nucleotides. Vent polymerase is slightly slower. 20-45 PCR cycles were performed depending on the purpose of the 
experiment. As a rule of thumb, fewer cycles were done if the product is a cDNA to be cloned.

\begin{tabular}{|c|c|}
\hline \multicolumn{2}{|c|}{ A typical PCR reaction mixture } \\
\hline $0.5-2 \mu \mathrm{l}$ template DNA & $1-200 n g$ \\
\hline $0.5 \mu \mathrm{l}$ forward primer & $100 \mu \mathrm{M}$ \\
\hline $0.5 \mu \mathrm{l}$ reverse primer & $100 \mu \mathrm{M}$ \\
\hline $2 \mu \mathrm{l}$ dNTP mix & $5 \mathrm{mM}$ \\
\hline $5 \mu l$ 10X PCR buffer & \\
\hline $0.25 \mu \mathrm{l}$ Vent polymerase & 0.5 Unit \\
\hline 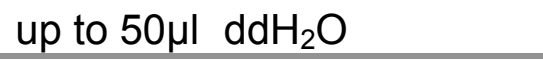 & \\
\hline
\end{tabular}

\begin{tabular}{cll}
\hline \multicolumn{2}{c}{ A typical PCR cycle } \\
\hline $94^{\circ} \mathrm{C}$ & $2-10 \mathrm{~min}$ & Initial denaturation \\
$94^{\circ} \mathrm{C}$ & $30 \mathrm{seC}$ & Denaturation \\
$60^{\circ} \mathrm{C}$ & $30 \mathrm{sec}$ & Annealing \\
$72^{\circ} \mathrm{C}$ & $30 \mathrm{sec}-4 \mathrm{~min}$ & Extension \\
$72^{\circ} \mathrm{C}$ & $5 \mathrm{~min}$ & Final extension \\
$4^{\circ} \mathrm{C}$ & $\infty$ & Storage \\
\hline
\end{tabular}

\subsubsection{Restriction digestion of DNA}

Restriction digestions were done either for analysis (i.e. southern blotting, colony screen) or for cloning of DNA. All restriction enzymes and their reaction buffers were purchased from NEB. Reaction mixtures were incubated at $37^{\circ} \mathrm{C} 2-16$ hours.

A typical restriction digest

DNA $1-5 \mu \mathrm{g}$

Restriction enzyme 10-20 units

10X Reaction buffer

BSA

$100 \mu \mathrm{g} / \mathrm{ml}$ final concentration

$\mathrm{ddH}_{2} \mathrm{O}$ 


\subsubsection{Ligation}

Prior to ligation, vector and insert DNA were restriction digested overnight. To prevent self ligation, ends of vector DNA were dephosphorylated using 5 units of alkaline phosphatase (NEB) for 15 minutes at $37^{\circ} \mathrm{C}$. For a typical ligation reaction $100 \mathrm{ng}$ of vector DNA and $3 X$ equimolar ratio of insert DNA were used. DNA was ligated at $16^{\circ} \mathrm{C}$ for 4 hours. TA cloning of PCR products into pcDNA3.1/N5-His-TOPO vector (Invitrogen) was performed according to manufacturer's instructions.

A typical ligation reaction

Vector DNA (i.e. 5kb pcDNA) 100ng

Insert DNA (i.e. 1.5kb PCR product) 100ng

T4 DNA ligase(NEB)

200units

10X Ligation Buffer(NEB)

$1 \mu \mathrm{l}$

$\mathrm{ddH}_{2} \mathrm{O}$

up to $10 \mu l$

\subsubsection{Transformation of chemically competent E.Coli}

For preparation of chemically competent E.Coli cells, $50 \mathrm{ml}$ of LB medium was inoculated with a bacterial colony grown overnight on an LB plate. Cells were grown at $37^{\circ} \mathrm{C}$ by shaking $300 \mathrm{rpm}$ until $\mathrm{OD}_{600}$ value reached 0.4 . Cells were spinned down 10 minutes at $4^{\circ} \mathrm{C}$ by centrifugation at 3000rcf. Pellet was resuspended in $10 \mathrm{ml}$ sterile ice cold $0.1 \mathrm{M} \mathrm{CaCl}_{2}$ and cells were kept on ice for $15 \mathrm{~min}$ to $4 \mathrm{~h}$. Cells were spinned down as before and the pellet was resuspended in $2 \mathrm{ml} \mathrm{CaCl}_{2}$. Sterile glycerol was added to an end concentration of $10 \%$. Cells were shock frozen by plunging 50 $100 \mu$ laliquots in liquid nitrogen. Aliquots were stored at $-80^{\circ} \mathrm{C}$ until use.

Prior to heat shock transformation, aliquots of competent cells were thawed on ice and mixed with a $2 \mu \mathrm{l}$ completed ligation reaction. After incubation on ice for $15 \mathrm{~min}$, heat shock was applied by incubating the cells at $42^{\circ} \mathrm{C}$ water bath for 60 seconds. Cells were immediately placed on ice and spread on an ampicillin containing LB plate. Before spreading to LB-Kanamycin plates, cells were incubated at $37^{\circ} \mathrm{C}$ in LB medium for $1 \mathrm{~h}$ by gentle shaking to enhance recovery. LB plates were incubated in $37^{\circ} \mathrm{C}$ incubator overnight until colonies appeared. 


\subsubsection{Expression and purification of recombinant proteins}

For bacterial expression of recombinant proteins, genes cloned into pET28 or pGEX vectors have been used. These vectors introduce an N-terminal hexa-histidine or GST tag to the cloned DNA, respectively. All proteins were expressed in BL21 strain E.coli cells.

A $50 \mathrm{ml}$ LB medium, containing $100 \mu \mathrm{g} / \mathrm{ml}$ ampicillin (pGEX) or $25 \mu \mathrm{g} / \mathrm{ml}$ kanamycin (pET28), was inoculated with the respective glycerol stock of BL21 cells. Cells were grown 16 hours at $37^{\circ} \mathrm{C}$ with $300 \mathrm{rpm}$ shaking. Next day in the morning, this starter culture was used to inoculate a 1t 2xYT medium with antibiotics. Cells were grown further 3-4 hours until they reached an $\mathrm{OD}_{600}$ value of 0.8 . Cells were induced to express the recombinant protein by addition of $0.5 \mathrm{mM}$ final concentration of IPTG to the culture medium. Protein expression was generally performed at $30^{\circ} \mathrm{C}$ for 4 hours at 200rpm shaking speed. An aliquot of cells before and after the induction were taken for the analysis of protein extracts by SDS-PAGE, in order to check the inducibility of the target protein expression. When induction is over, bacterial cells were pelleted by spinning down at 3000 rcf for 15 minutes at $4^{\circ} \mathrm{C}$. Pellet was resuspended in $40 \mathrm{ml}$ ice cold PBS containing $1 \mathrm{mM}$ PMSF and a few milligrams of lysozyme was added with the tip of a spatula. Bacterial resuspension was mixed and incubated further 15 minutes on ice for the digestion of the cell walls. Cells were lysed by sonicating 3-4 times (each 30 seconds) on ice with a SONOPLUS sonicator from Bandelin using $50 \%$ duty cycle and $60 \%$ power settings. Triton X-100 was added to a final concentration of $1 \%$ and cell extracts were incubated for 15 minutes at $4{ }^{\circ} \mathrm{C}$ on a rotating wheel. Some DNAsel could be added during this step to break the viscosity of the extract, however it is not essential. Finally, cell lysate was cleared from debris by spinning down the resuspension at 25000rcf. An aliquot of the cell lysate and pellet were taken after this step and analyzed by SDS-PAGE in order to check the solubility of the protein.

Cleared cell lysate was transferred to a $50 \mathrm{ml}$ falcon tube and $0.2-1 \mathrm{ml}$ of PBS washed glutathione or Ni-NTA bead slurry was added. For binding of the recombinant proteins to the affinity matrices, falcon tubes with lysates were incubated 2 hours at $4^{\circ} \mathrm{C}$ on the rotating wheel. Beads were washed 3 times 10 minutes with ice cold PBS. In the case of histidine tagged proteins, $10-20 \mathrm{mM}$ of imidazole was included during the final washing step. After the washing steps, concentrations of the recombinant 
proteins were measured by Bradford assay and their purity was analyzed by SDSPAGE. Recombinant proteins bound to beads were used frequently in pull down assays from protein extracts.

When required, GST fusion proteins were eluted immediately after the wash steps by incubating the beads with $25 \mathrm{mM}$ reduced glutathione, 60 minutes at RT on the rotating wheel. A second 30 min elution was usually required to obtain the residual protein. Histidine tagged proteins were eluted by incubating the beads with 250 $500 \mathrm{mM}$ imidazole, $60 \mathrm{~min}$ at $4^{\circ} \mathrm{C}$ on the rotating wheel.

\subsubsection{Generation of antisera against stonin 1 and stonin 2}

The biochemical and cytogenetic characterization of stonin 1 and stonin 2 proteins and their interaction partners requires antibodies that specifically recognize stonins. Therefore, creation of good antisera against stonins was essential for the success of this project.

Two different immunization approaches have been pursued for the production of stonin 2 antisera; i) immunization of rabbits with a purified GST-Stonin 2 amino terminal domain (NT) fusion protein or ii) immunization with chemically synthesized stonin 2 peptides.

GST-stonin2NT fusion protein was generated by cloning the human stonin2 nucleotide sequence coding for the first 555 residues into pGEX4T1 vector. Although expression of GST-stonin $2^{\mathrm{NT}}$ fusion protein was induced quite well in bacterial cells, it was not soluble. In order to extract the protein from the insoluble fraction, cells were lysed by sonicating them in the presence of $3 \mathrm{M}$ urea. The lysate was dialysed against 2I PBS overnight to reduce the urea concentration to less than $100 \mathrm{mM}$. After binding to the beads and washes, the fusion protein was eluted using $25 \mathrm{mM}$ glutathione as described before. The eluate was dialysed against PBS to remove glutathione. 200 $\mathrm{\mu g}$ aliquots were shock-frozen in liquid nitrogen after addition of $10 \%$ glycerol. Frozen antigen solutions were sent to Eurogentec SA (Belgium) for immunization of two rabbits. An immunization scheme suggested by the company was used. Five bleeds were acquired from each rabbit before terminating the program. Sera from both rabbits were quite specific for stonin 2 and had a high titer as shown in Figure 2.1. 


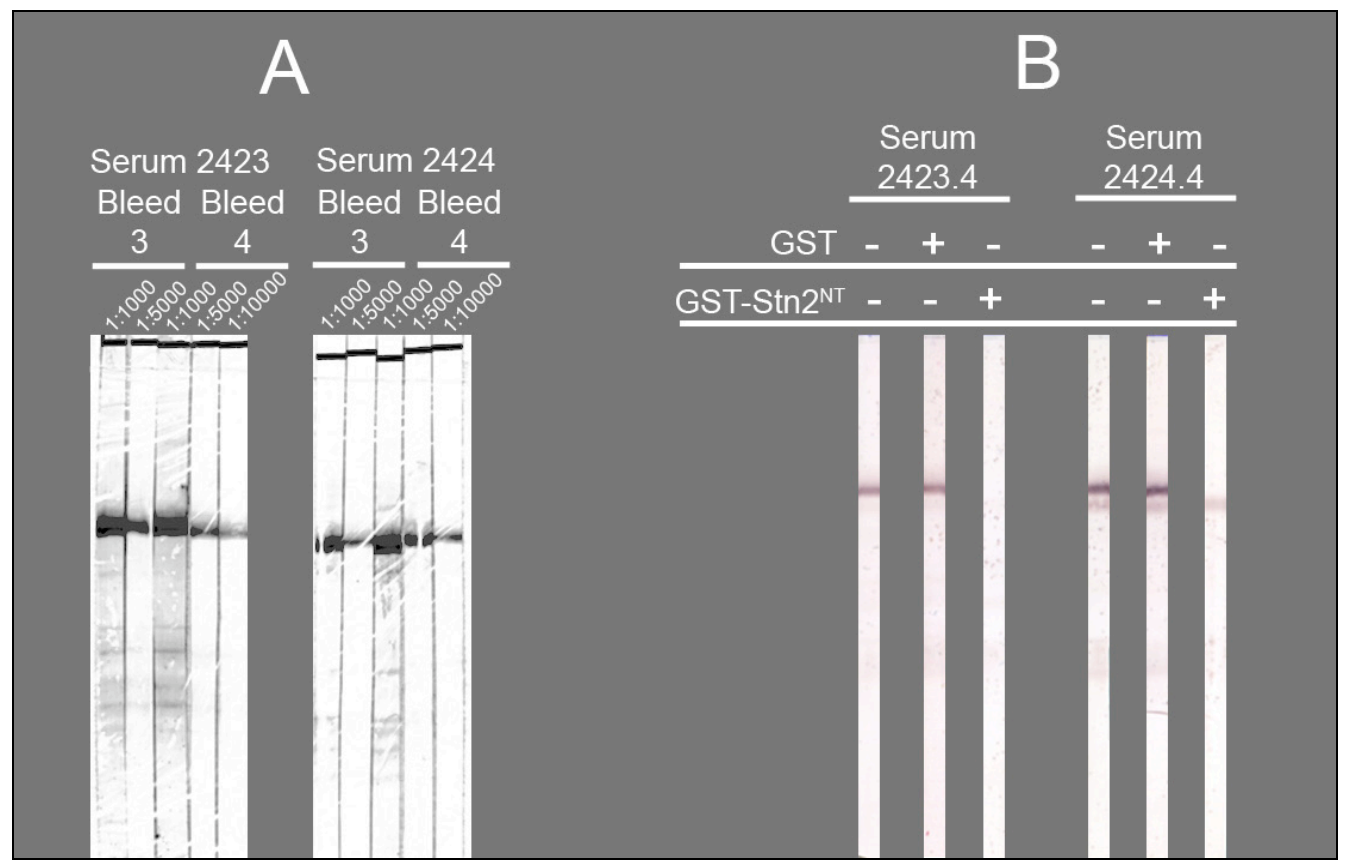

Figure 2.1 Testing of stonin 2 antisera. (A) Stonin 2 antisera were tested using strips of RBE

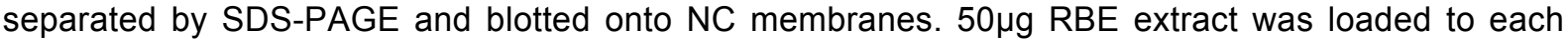
well. Stonin 2 antisera had a high titer and specificity as even 1:10000 dilution was sufficient to detect a single band of about 120-130 kDa molecular weight. (B) Preincubation of stonin 2 antisera with recombinant GST-Stonin2 ${ }^{\mathrm{NT}}$ results in disappearance of the recognized band. In contrast, GST preincubation had no effect.

In the second approach, two rabbits were immunized by a mixture of two synthetic peptides. Both peptides were chosen from a mouse stonin 2 peptide sequence such that the selected sequence was highly conserved between mouse and human stonin 2. The immunization program was administered by Eurogentec SA as before.

\begin{tabular}{lll}
\hline Name & Peptide sequence & Residues in mouse stonin2
\end{tabular}

EP023453 or \#53 $\quad \mathrm{NH}_{2}$-SFDDSGKSQPHPDAIC-CONH ${ }_{2} \quad 365-379$

EP023454 or \#54 $\quad \mathrm{NH}_{2}$-CRVTYKEKKKYQPKPA-CONH $\mathrm{H}_{2} \quad 500-514$

At the end of the immunization program, antibodies were affinity purified from the sera using peptides coupled to an affinity matrix. Only antibodies affinity-purified by peptide \#54 could detect stonin2 in western blots and immunofluorescence experiments, however the titer was at least 10 times lower compared to the stonin 2 antisera (\#2423 and \#2424) described above.

In order to develop antisera against stonin1, we decided to use an N-terminal 6-His tagged full length stonin1 as antigen for two reasons. Antisera developed against 
GST fusion proteins cross react with GST. In addition, histidine-tagged proteins can be eluted from the affinity matrix almost quantitatively after affinity purification.

Full length mouse stonin 1 cDNA sequence was cloned into pET28a vector for expression of a hexa-histidine tagged fusion protein. Unfortunately, induction of stonin 1 expression was very inefficient in E.coli under several different conditions tested. For purification of stonin 1, bacterial cultures (in $4 \mathrm{I}$ of 2xYT medium) were induced to express stonin 1 at $20^{\circ} \mathrm{C}$ overnight. $6 \mathrm{His}$-stonin 1 fusion protein was purified and eluted from Ni-NTA beads as before. $0.75 \mathrm{mg}$ of protein, obtained from 4I culture, was dialysed against PBS and $100 \mu$ laliquots were shock-frozen in liquid nitrogen. Two rabbits were immunized by Eurogentec SA according to the company's schedule and antisera were shipped to us. Stonin 1 antisera did not recognize any specific protein bands of appropriate molecular weight range (80-90 $\mathrm{kDa}$ ) in RBE or other tested tissues (data not shown). However, they were able to recognize exogeneously expressed stonin 1 in transfected cells tested by western blotting (data not shown) and immunofluorescence staining (Figure 2.2).

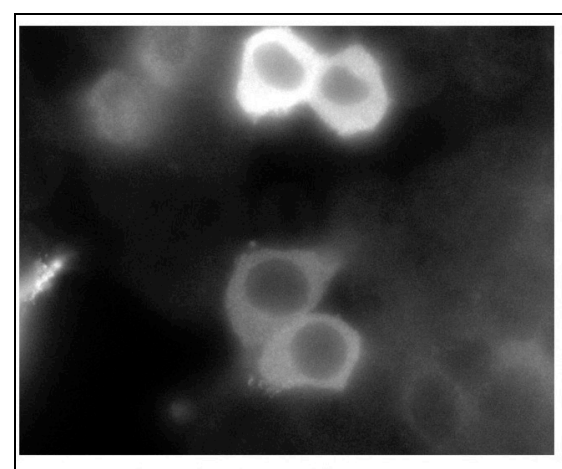

stonin1 antiserum

$85.31: 2000$

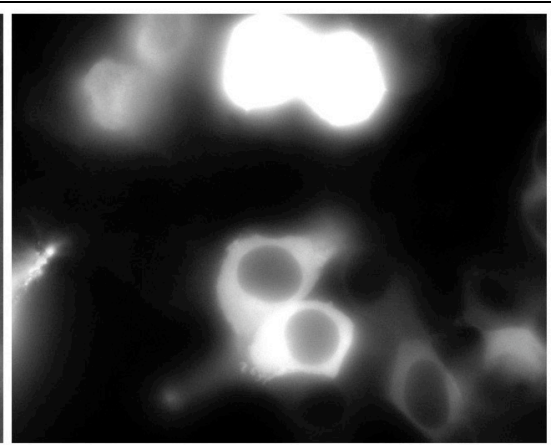

EGFP

Figure 2.2 Testing of stonin 1 antisera by immunofluorescence microscopy. N1E-115 cells were transfected with EGFP-Stonin 1 and immunostained with stonin1 antibodies. 1:2000 dilution of bleed 85.3 is able to detect mild to low level stonin 1 expression.

\subsubsection{Preparation of genomic DNA from cells and animal tissue}

Genomic DNA for screening embryonic stem cell colonies was prepared from ES cells cultured in 24-well plates. For genotyping of transgenic animals, DNA was obtained from tail tips or pieces of ear obtained after ear punching.

As the first step, $500 \mu$ l of SNET buffer was added to the cell pellets or animal tissues in $1.5 \mathrm{ml}$ microfuge tubes and tubes were thoroughly vortexed. After the addition of proteinase $\mathrm{K}\left(200 \mu \mathrm{g} / \mathrm{ml}\right.$ final concentration), tubes were incubated at $56^{\circ} \mathrm{C}$ in a 
rocking incubator or water bath $2 \mathrm{~h}$ to overnight. No clumps of tissue should be visible after this point. Next, $500 \mu \mathrm{l}$ of phenol:chloroform:isoamylalcohol (25:24:1) solution was added to the dissociated tissue solution. Tubes were vortexed for $30 \mathrm{~s}$ and centrifuged 5-10 min at maximum speed in a table-top centrifuge. Hair pieces and small clumps of tissue should partition into the organic phase. Genomic DNA was gently recovered together with the aqueous upper phase. In order to precipitate

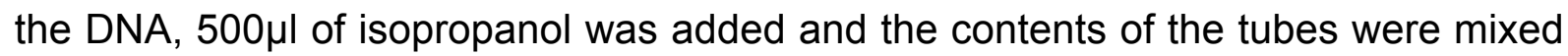
by gently turning upside down several times. After this, DNA is usually visible as a white fluffy precipitate. The DNA precipitate was reisolated by centrifugation at maximum speed, and washed, first with $500 \mu \mathrm{l} 100 \%$ ethanol, then with $70 \%$ ethanol. Following removal of the ethanol, DNA pellets were dried by incubating the tubes at $37^{\circ} \mathrm{C}$ for $15 \mathrm{~min}$ with their lids left open. Finally, the DNA pellet was dissolved in 50$200 \mu$ of TE buffer or $\mathrm{ddH}_{2} \mathrm{O}$ by means of incubating at $37^{\circ} \mathrm{C}$ and vortexing intermittently.

\subsubsection{Southern hybridization}

Restriction digestion of genomic DNA and southern hybridization are the standard methods for screening homologous recombination events in ES cell colonies. Upon insertion of the targeting vector into the genomic locus via homologous recombination, some restriction sites are deleted or new sites are introduced. This additional locus engineering is done to ease colony screening afterwards. For example, in the case of the stonin 2 targeting vector, a novel HinDIII restriction site was introduced.

$4 \mu \mathrm{g}$ of genomic DNA samples isolated from ES cells cultured in 24-well plates were digested with 20 units of HinDIII enzyme overnight in reaction volumes of $20 \mu \mathrm{l}$. At the end of the restriction digests, the DNA solutions should not be viscous anymore. Digested DNA samples were separated by running through a $0.7 \%$ agarose gel (16cm length) for 4-5 $\mathrm{h}$ at low voltage (4-5 Volts per $\mathrm{cm}$ ). Gel was stained in $\mathrm{EtBr}$ bath and photographed for documentation. Next, the gel was briefly rinsed in $\mathrm{ddH}_{2} \mathrm{O}$ and soaked in $10 \mathrm{gel}$ volumes of denaturation solution with constant gentle agitation (on a rotatory platform) for $45 \mathrm{~min}$ at RT. After this, the gel was rinsed with $\mathrm{ddH}_{2} \mathrm{O}$ and soaked in $10 \mathrm{gel}$ volumes of neutralization buffer for $30 \mathrm{~min}$ and again with 15 min as before. Finally, the gel was equilibrated with 20X SSC (transfer solution) for $30 \mathrm{~min}$. 
The Southern transfer apparatus was assembled as shown in Figure 2.3. The agarose gel was layered upside down over 2 prewetted 3MM filter papers whose ends were soaked in 20X SSC buffer. Hybond-N membrane, cut exactly to fit the dimensions of the gel, was wetted first in $\mathrm{ddH}_{2} \mathrm{O}$ then in 20X SSC buffer and layered on top of the gel. Next 3 wet filter papers and a stack of smaller sized dry paper towels were layered over the membrane. Any air bubbles formed during the layering steps should be removed by rolling a glass pipette over. Finally, a 1-1.5 kg weight was applied which was equally distributed throughout the gel by the help of a glass plate. The transfer of DNA onto the nylon membrane proceeds overnight.

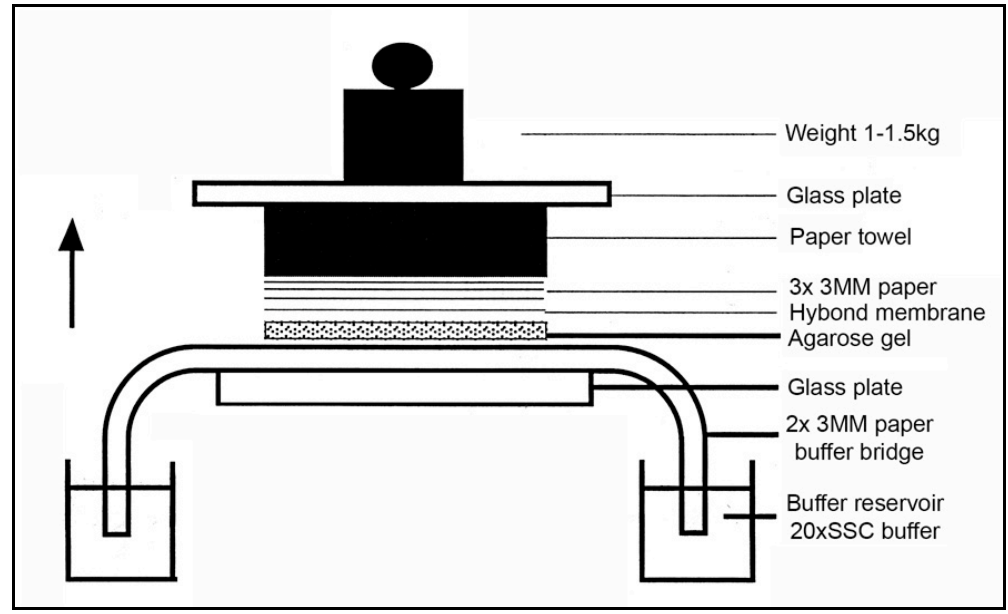

Figure 2.3 Southern transfer setup.

Next morning, DNA transfer setup was disassembled and the lanes of the agarose gel were marked on the membrane using a pencil. The membrane was briefly dried on a filter paper and DNA was cross linked to the membrane in a UV cross-linker with an illumination of $0.12 \mathrm{~J} / \mathrm{cm}^{2}$.

PCR amplified DNA fragments have been used as probes for Southern hybridization. $25 n$ purified DNA probe was labeled with [ $\left.{ }^{32} \mathrm{P}\right] \mathrm{dCTP}$ using Rediprime ${ }^{\mathrm{TM}}$ II random prime labeling kit (Amersham) according to the manufacturers instructions. Prior to hybridization, probes were denatured for 5 mins at $95^{\circ} \mathrm{C}$ and chilled on ice. The amount of probe used in each experiment was determined according to the area of the membrane and the kit instructions (i.e. $2 \mathrm{ng} / \mathrm{ml}$ ).

Rapid-hyb hybridization buffer (Amersham) was used in all southern hybridization experiments with a volume of $0.125 \mathrm{ml}$ per $\mathrm{cm}^{2}$ of membrane. An initial $2 \mathrm{~h}$ prehybridization/blocking step without DNA probe preceded the overnight hybridization step at $65^{\circ} \mathrm{C}$. Membranes were washed once for 20 min in $50 \mathrm{ml} 2 \mathrm{x}$ 
SSC, $0.1 \%(\mathrm{w} / \mathrm{v})$ SDS at RT and twice for $15 \mathrm{~min}$ in $50 \mathrm{ml} 0.2 \times \mathrm{SSC}, 0.1 \%(\mathrm{w} / \mathrm{v})$ SDS at $65^{\circ} \mathrm{C}$. Membrane was dried and exposed to a phosphoimager screen for $2 \mathrm{~h}$. After scanning of the screens, MW of the hybridizing DNA fragments was determined using the photographs of $\mathrm{EtBr}$ stained gels.

\subsubsection{Immunoprecipitation}

A premixed slurry of protein A/protein $G$ agarose beads (Santa Cruz) was used for coupling of both monoclonal and polyclonal antibodies. $2-20 \mu \mathrm{l}$ of antiserum or purified antibody was coupled to $10-20 \mu \mathrm{l}$ of beads for $1 \mathrm{~h}$ in $500 \mu \mathrm{l}$ antibody solution containing $0.1 \%$ Triton $\mathrm{X}-100$ by continuously mixing in a rotating wheel. Coupling step also serves as a blocking step since antibody solution contains $2 \%(\mathrm{w} / \mathrm{v}) \mathrm{BSA}$. Beads were washed 3 times with PBS and once with the IP buffer (the buffer in which the particular protein extracts were prepared, typically 20mM HEPES pH7.4, 150mM $\mathrm{NaCl}, 1 \mathrm{mM}$ PMSF, $2 \mathrm{mM} \mathrm{MgCl} 2,1 \%$ Triton X-100). Antibody coupled beads were added to the precleared protein extracts and incubated at $4^{\circ} \mathrm{C}$ in a rotating wheel for 1-2 h. Beads were washed 3 times for 10 min with IP buffer. During colP experiments, beads were washed very gently just by flipping the tubes a couple of times. All traces of buffer were removed from the beads using a Hamilton syringe and $40-80 \mu \mathrm{l}$ of SDS-PAGE sample buffer was added over the beads to denature and elute the bound proteins and antibodies. Tubes were further incubated at $95^{\circ} \mathrm{C}$ for 5 min and then the samples were analyzed by SDS-PAGE or stored frozen at $-20^{\circ} \mathrm{C}$.

\subsubsection{GST pull-down assays}

Even though, colP experiments yield more dependable data, it is not always possible to perform them for technical reasons, such as absence of good antibodies against one of the proteins. Interactions between proteins can also be tested by means of a, so called, GST pull-down assay. In this assay, one of the putative interacting partners is purified from bacterial cells as a recombinant fusion protein (as explained in section 2.2.2). In the course of this project, several GST pull-down assays have been done.

Recombinant proteins bound to glutathione-agarose beads (Novagen) were used immediately after purification or stored on ice no longer than one day before usage.

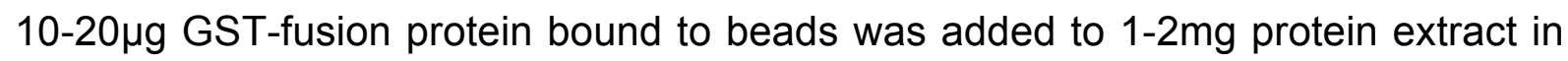
$1 \mathrm{ml}$ buffer(typically $20 \mathrm{mM}$ HEPES pH7.4, $150 \mathrm{mM} \mathrm{NaCl}, 1 \mathrm{mM} \mathrm{PMSF}, 2 \mathrm{mM} \mathrm{MgCl}$, 
$1 \%$ Triton $\mathrm{X}-100)$ and incubated for $1-2 \mathrm{~h}$ at $4^{\circ} \mathrm{C}$ on a rotating wheel. The beads were washed 3 times for 10 min with the same buffer and once with PBS. SDS-PAGE sample buffer was added over the beads to elute the proteins. The samples were separated by an SDS gel and analyzed by Coomassie Blue staining or Western blotting.

\subsubsection{SDS-PAGE}

SDS-PAGE (Polyacrylamide gel electrophoresis) is a widely used biochemical technique for separation and analysis of protein samples. A mini protean II gel casting apparatus (Biorad) has been used for preparation of $8-12 \%$ gels according to the Table 2.8 below.

\begin{tabular}{llllll}
\hline \multicolumn{2}{c}{ Stacking gel } & \multicolumn{5}{c}{ Separating Gel } \\
\hline Percentage & $4.5 \%$ & Percentage & $8 \%$ & $10 \%$ & $12 \%$ \\
Acrylamide/bis-acrylamide & $1.5 \mathrm{ml}$ & Acrylamide/bis-acrylamide & $2.7 \mathrm{ml}$ & $3.3 \mathrm{ml}$ & $4.0 \mathrm{ml}$ \\
& & & & & \\
$4 \mathrm{X}$ stacking gel buffer & $2.5 \mathrm{ml}$ & $4 \mathrm{X}$ separating gel buffer & $2.5 \mathrm{ml}$ & $2.5 \mathrm{ml}$ & $2.5 \mathrm{ml}$ \\
$10 \%(\mathrm{w} / \mathrm{v})$ APS & $50 \mu \mathrm{l}$ & $10 \%(\mathrm{w} / \mathrm{v})$ APS & $50 \mu \mathrm{l}$ & $50 \mu \mathrm{l}$ & $50 \mu \mathrm{l}$ \\
$\mathrm{ddH}_{2} \mathrm{O}$ & $5.9 \mathrm{ml}$ & $\mathrm{ddH}_{2} \mathrm{O}$ & $4.8 \mathrm{ml}$ & $4.2 \mathrm{ml}$ & $3.5 \mathrm{ml}$ \\
TEMED & $15 \mu \mathrm{l}$ & TEMED & $5 \mu \mathrm{l}$ & $5 \mu \mathrm{l}$ & $5 \mu \mathrm{l}$ \\
\hline
\end{tabular}

Table 2.8 Preparation of SDS gels

Proteins samples in $1 \mathrm{X}$ SDS-PAGE sample buffer were denatured by heating at $95^{\circ} \mathrm{C}$ for 5-10 min. 10-40 $\mu$ l of the sample was loaded per well. Proteins were separated by running them at $150 \mathrm{~V}$ until separating gel was reached, and further on at 200-250V for 1-1.5 h. For analysis, gels were stained 20-30 min in coomassie blue staining solution and destained 30 min to overnight in destaining solution. Alternatively, gels were immediately used for immunoblotting.

\subsubsection{Immunoblotting}

Western blotting or immunoblotting is the procedure whereby protein samples separated according to their molecular weights by SDS-PAGE are transferred and cross-linked to a membrane. FastBlot B44 blotting apparatus (Biometra) has been used to apply an electric field to the gels that facilitates transfer of negatively charged proteins.

The separating gel, nitrocellulose membrane and 6 3MM filter papers (cut to exactly fit the dimensions of the gel) were wetted in immunoblot transfer buffer and stacked 
according to the figure 2.4 below. Any remaining air bubbles remaining between the layers were squeezed out by rolling a glass pipette over them. A continuous current of $1 \mathrm{~mA}$ per $\mathrm{cm}^{2}$ of gel (i.e. $40 \mathrm{~mA}$ per gel) was applied for 1.5-2 h. 2-3 kgs of weight was placed over the blotting apparatus during the transfer.

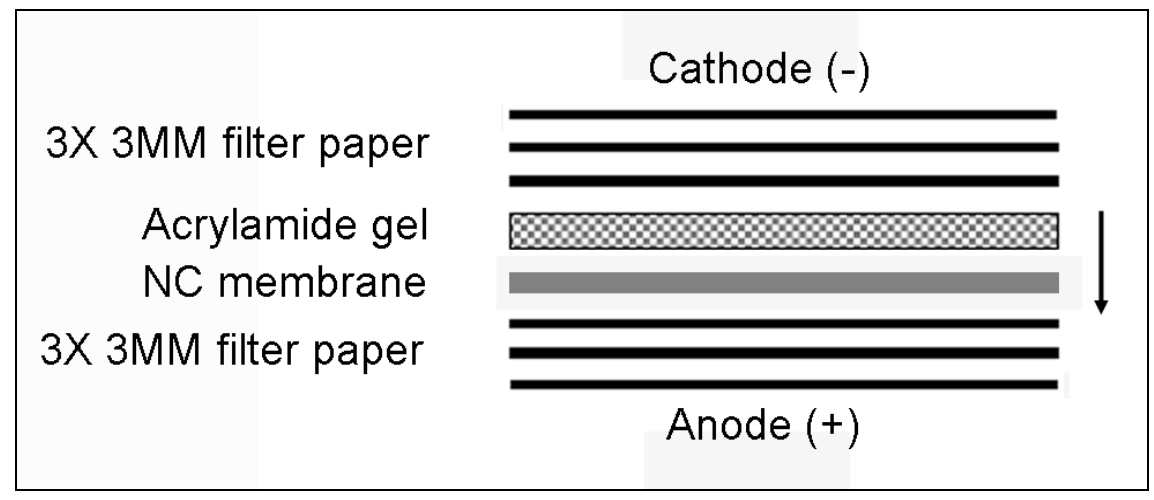

Figure 2.4 Setup for electric field assisted Western blotting.

At the end of the transfer the membranes were separated from the gels and stained for 5 min with Ponceau S solution and destained several times. Protein molecular weight marker lane was marked with a ball point pen and the membranes were scanned for documentation.

Membranes were blocked with $10 \mathrm{ml}$ blotto for $1 \mathrm{~h}$ at room temperature (RT) by gently shaking on a rotatory platform. After rinsing with TBS buffer, membranes were decorated with primary antibodies for $2 \mathrm{~h}$ at RT or overnight at $4^{\circ} \mathrm{C}$. Next, they were washed 3 times 10 min with blotto and decorated with HRP conjugated secondary antibodies in $10 \mathrm{ml}$ blotto for $1 \mathrm{~h}$ at RT. After washing 3 times with blotto and rinsing once in TBS, labeled protein bands were detected by ECL chemiluminescence detection system from Amersham.

For quantification of immunoblot results, ${ }^{125}$ I labeled protein A was used. Protein A has a high affinity for polyclonal rabbit antibodies but does not bind to mouse monoclonal antibodies efficiently. Therefore, after using monoclonal antibodies as primary antibody, membranes were redecorated with rabbitamouse antibodies for one hour to boost the signal. After the washing steps, membranes were exposed to a phosphoimager screen and scanned by a phosphoimager.

\subsubsection{Mouse embryonic stem cell culture and electroporation of the targeting vector}


Mouse embryonic stem cells are cultured in a similar fashion to other common adherent cell lines. However, ES cells are totipotent cells with a tendency to differentiate to other lineages and loose their totipotency if not cultured with care. In this project, the end reason for culturing ES cells was the generation of germ-line mouse chimeras and thus, preservation of the undifferentiated state was vital.

ES cell lines are derived from the inner cell mass of blastocysts from 3.5 day-old mouse embryos. Isolation and characterization of totipotent ES cell lines is a complicated procedure requiring experience. Therefore, a previously characterized and commonly used cell line (ES 14) was obtained from Dr. Peter Schu. These cells were derived from agouti colored SV129/Ola inbred mouse strain.

ES cells were cultured on a feeder layer of primary mouse embryonic fibroblasts (PMEF) in the presence of special ES cell media with supplements in order to suppress differentiation. Murine leukemia inhibitory factor (LIF) is one of the crucial supplements and it inhibits differentiation. In addition, the serum to be used has to be tested for a possible tendency to cause differentiation. The composition of the ES cell media that was used is demonstrated in Table 2.9 below. After preparation, it was filtered and used within 2-3 weeks. ESGRO-LIF, the most expensive component of the medium, was added separately whenever needed.

\begin{tabular}{llll}
\hline Component & Amount & Supplier & Catalogue/Lot No \\
\hline D-MEM 4500mg/L glucose with L-Glutamine & $500 \mathrm{ml}$ & GIBCO & $41965-039$ \\
15\% Foetal Calf Serum & $80 \mathrm{ml}$ & GIBCO & $10270-106$ \\
L-Glutamine, 200mM & $5 \mathrm{ml}$ & GIBCO & $25030-024$ \\
Penicillin(10000U/ml)/Streptomycin(10mg/ml) & $5 \mathrm{ml}$ & GIBCO & $15140-122$ \\
Na-Pyruvate, 100mM & $5 \mathrm{ml}$ & GIBCO & $11360-039$ \\
Non-essential aminoacids 100X & $5 \mathrm{ml}$ & GIBCO & $11140-035$ \\
2-Mercaptoethanol, 50mM & $750 \mu \mathrm{l}$ & GIBCO & $31350-010$ \\
ESGRO(LIF Factor) & $1000 \mathrm{units} / \mathrm{ml}$ & Chemicon & ESG1106 \\
\hline
\end{tabular}

Table 2.9 Composition of the ES culture media

One vial of $\mathrm{E} 14$ cells from $8^{\text {th }}$ passage were thawed in a $37^{\circ} \mathrm{C}$ water bath and the cells were pelleted by centrifugation at $200 \mathrm{~g}$ and resuspended in ES cell medium. Cells were seeded into three $25 \mathrm{~cm}^{2}$ flasks previously coated with mitotically 
inactivated PMEF cell layer. Next day, the medium was changed to clear the dead cells. 2 days after, the first colonies started to appear and medium became yellow (acidic). The medium was changed and cells were cultured another 3 days until they were confluent and the colonies became larger. Undifferentiated ES cell colonies have a smooth surfaced three-dimensional appearance with clear refractal borders separating them from feeder layer. Cells from differentiating colonies usually start to grow in monolayers like fibroblasts. The ES cells were continuously monitored during the culturing period and they were always fed by changing $80 \%$ of the medium whenever it turned yellow (repeated every $24 \mathrm{~h}$ initially, but as frequent as every $6 \mathrm{hs}$ when the cells became confluent). 6 days after they were thawed, the ES cells reached the desired confluency and were ready for electroporation.

One day before the electroporation linearized targeting vectors were prepared.

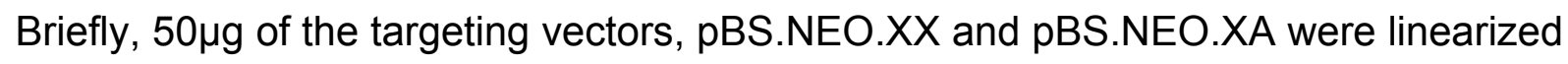
by restriction digestion with $\mathrm{Xhol}$ and Apal enzymes respectively. DNA was extracted first with phenol/chloroform and then with chloroform, and precipitated using absolute $\mathrm{EtOH}$ at $-80^{\circ} \mathrm{C}$. Pellets were washed twice with $70 \% \mathrm{EtOH}$, air dried, redisolved in $200 \mu$ l PBS and stored at $4^{\circ} \mathrm{C}$ until use.

On the day of electroporation, ES cells from three confluent flasks were trypsinized and recovered by centrifugation. Cells were washed once with serum free medium and twice with PBS. Cells were resuspended in $1 \mathrm{ml}$ PBS and counted. Around 15-20 million cells were obtained from each flask. $800 \mu l$ of the cell suspension was mixed with $200 \mu$ l linearized DNA solution or buffer and kept on ice for $5 \mathrm{~min}$. After electroporating the cells once at $250 \mu \mathrm{F}$ (GenePulserll Biorad), cuvettes were kept at RT for 2 min and then the cells were seeded into two $10 \mathrm{~cm}$ dishes coated with inactivated PMEF feeder layer and kept in incubator. Next day, the dishes were confluent as most cells had attached to the dish and the few dead cells were cleared by changing the medium. The ES cells were selected for drug resistance starting 24 $\mathrm{h}$ after electroporation using 208 $\mu \mathrm{g} / \mathrm{ml}$ Geneticin G418 (GIBCO, 10131-019). Medium was changed every $12 \mathrm{~h}$ as it turned yellow quickly. Roughly $48 \mathrm{~h}$ after the selection started, there was an extensive cell death. Dying cells were cleared by changing the medium. Drug resistant ES cell colonies started to become apparent 6-7 days after the electroporation. No colonies from the mock transfected ES cells had survived and these dishes were discarded. There were about 500 surviving ES cell colonies per 
$10 \mathrm{~cm}$ dish from the ES cell batch transfected with pBS.NEO.XX vector but only very few colonies (10-20) from the pBS.NEO.XA batch (also discarded later) survived. Colonies were ready to be picked 9 days after the electroporation.

Geneticin resistant ES cell colonies were picked under an inverted light microscope using a $200 \mu l$ pipette (Gilson) and bore yellow tips. Cells were washed twice with PBS and kept in PBS during the picking procedure. Well separated, undifferentiated and relatively large colonies were picked up carefully and placed in the wells of a 96well plate containing 50 $\mu$ trypsin-EDTA solution (GIBCO, 25200-056). Colonies were kept in trypsin solution at RT for maximum 30 min until 10-12 colonies were collected. The colonies were incubated at $37^{\circ} \mathrm{C}$ for 3-4 min for dissociation. ES cells were further dispersed by trituration and seeded into the wells of PMEF coated 24-well plates. Picking of ES cell colonies was a time consuming task and it was not desirable to keep ES cells in PBS for long periods of time. Therefore, after picking colonies for 30 minutes from the first dish, full medium was replaced and the second dish was used for picking of the next 10-12 colonies. A total of 150 colonies have been picked in two days.

Picked ES cells were further grown in 24 well plates for 5-7 days in selective medium until they reached confluence. Confluent clones were trypsinized, and 2/3 of the cells were frozen in freezing medium ( $80 \%$ ES cell medium+10\%FCS and 10\%DMSO). During freezing, the cells were first incubated at $-20^{\circ} \mathrm{C}$ for $1-2$ hours, then overnight at $-80^{\circ} \mathrm{C}$ and finally stored frozen in liquid $\mathrm{N}_{2}$ tanks. The remaining $1 / 3$ of the cells were cultured for a few days on gelatinized 24 well plates (without feeder layer). These cells were finally harvested by trypsinization and their genomic DNA was isolated.

\subsubsection{Primary mouse embryonic fibroblasts culture and mitotic inactivation}

Before the discovery of LIF as a differentiation suppressing factor, ES cells were cultured over a layer of mitotically inactivated PMEF cells to preserve totipotency. Nowadays, ES lines exist (i.e. R1) that do not require a PMEF layer but can be cultured in gelatinized culture dishes in the presence of LIF. PMEF cells are isolated by dissociating fibroblast rich tissues of 12.5-13.5 days old mouse embryos by trypsinization. They have to be resistant against geneticin and free of mycoplasma contamination to be appropriate as a feeder layer for ES cells. 
We received several frozen vials of PMEF cells with passage number 3 from Dr. Peter Schu. These PMEF cells were cultured in $15 \mathrm{~cm}$ dishes for $2-3$ days until they became confluent and then they were split 1:3 and cultured further for 3-4 days. Cells were grown in ES cell medium without LIF. Culture dishes were coated for 30 minutes at RT by incubating with $0.1 \%(w / v)$ gelatin (porcine skin gelatin type $A$, Sigma) dissolved in sterile $\mathrm{dd}_{2} \mathrm{O}$. Mitotic inactivation was carried out by incubating the cells with media containing $10 \mu \mathrm{g} / \mathrm{ml}$ mitomycin $\mathrm{C}$ for $3 \mathrm{~h}$. Mitotically inactivated cells were seeded at a density of $5-10 \times 10^{4}$ cells per $\mathrm{cm}^{2}$ one day before seeding of the ES cells.

\subsubsection{Construction of stonin 2 locus targeting vector}

The initial step in generation of a stonin 2 targeting construct was cloning of the mouse stonin 2 cDNA sequence. Human stonin 2 cDNA sequence was entered as a query and the mouse EST (expressed sequence tag) database at NCBI web site was scanned for similar sequences using the nucleotide BLAST program. Several EST sequences that matched 5' and 3' ends of human stonin 2 as well as mouse stonin1 full cDNA sequence were identified. Using EST sequences BF149603 (5' end) and BI144276 (3' end), primers were designed for RT-PCR amplification of mouse stonin 2. Cloning and sequencing of a partial mouse stonin 2 partial sequence showed that our existing human stonin 2 sequence lacked 2 nucleotides in the 5' part of the gene which resulted in a frame shift change of about 200 amino acids in the NT sequence. Human stonin 2 sequence was corrected accordingly. For screening of a mouse genomic DNA library, a 1.9 kb cDNA fragment (using primers Pr-4 and Pr-8) was cloned.

For efficient homologous recombination during gene/locus targeting, DNA sequences of the regions of homology between the targeting vector and genomic DNA have to be identical. For this reason, genomic DNA used for the generation of targeting construct has to be obtained from the same strain of mice from which ES cells were derived. We have used RZPD's (Deutsches Ressourcenzentrum für Genomforschung $\mathrm{GmbH}$, Berlin) library screening service to scan a mouse cosmid genomic DNA library (library number 121) using Pr-8/Pr-4 probe. The genomic DNA used in this library was cloned from the 129/ola strain mice that is identical to the strain ES.14 cells were derived from. 
As a result of screening, three positive cosmid clones were identified; MPMGc121J03406Q2, MPMGc121020316Q2 and MPMGc121M09670Q2, henceforth referred to as cosmid $\mathrm{J}, \mathrm{O}$ and $\mathrm{M}$ respectively. Presence of Exon 4 sequence in the cosmids $\mathrm{J}$ and $\mathrm{O}$ was confirmed by PCR amplification, partial sequencing (data not shown) and southern hybridization (Figures 2.5 and 2.6). Cosmid $\mathrm{M}$ was found to be a false positive as it did not contain any sequences related to mouse stonin 2 . Cosmid $\mathrm{O}$ was selected for further cloning procedures.

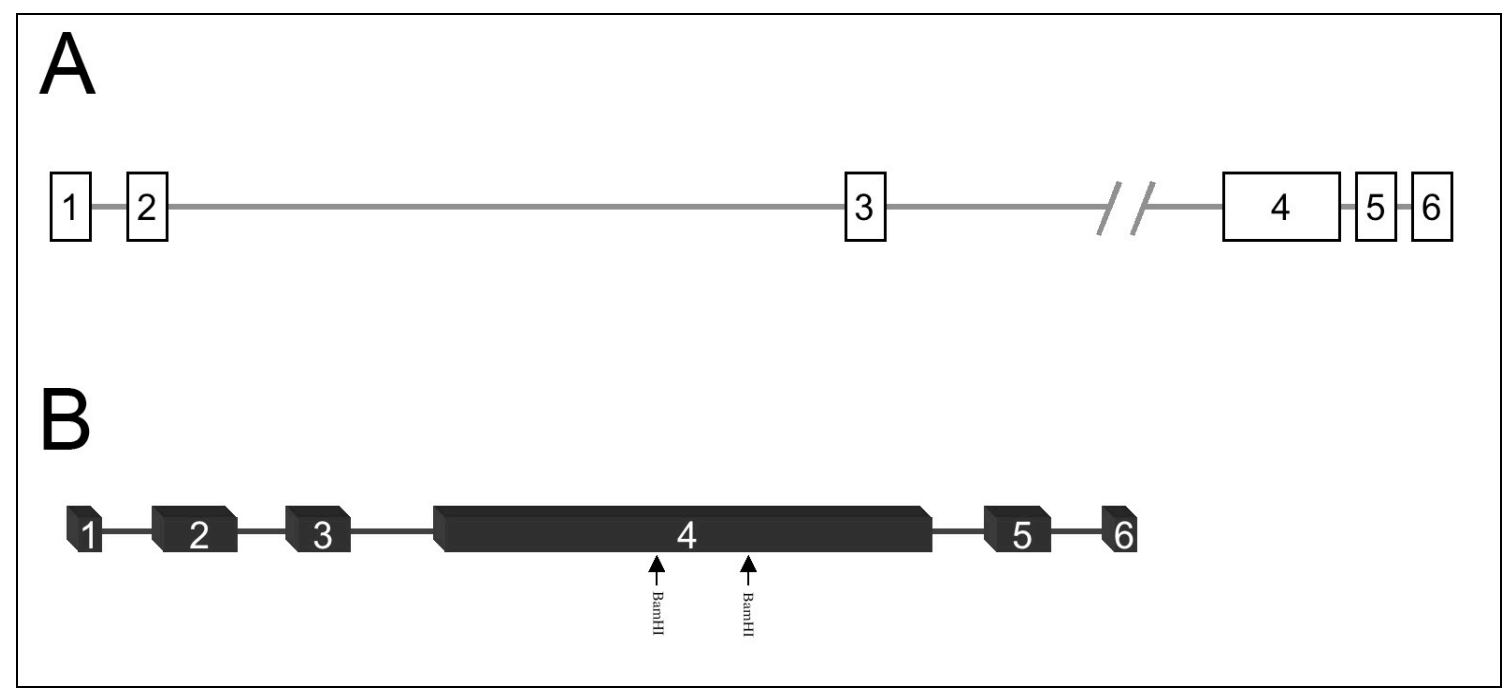

Figure 2.5 Genomic organization of mouse stonin 2 locus. (A) Mouse stonin2 gene (coding exons) is spread over a $105 \mathrm{~kb}$ genomic locus on chromosome 12 (between nucleotides 86245091 and 86350303). It consists of 6 coding exons and the first two and last three exons are clustered close to each other. Distances between depicted exons have been drawn to reflect the original ratios. (B) Schematic diagram indicating the relative length of exons encoding stonin 2. Exon1 (187), Exon2 (88-364), Exon3 (365-562), Exon4 (563-2401), Exon5 (2402-2604), Exon5 (2605-2688). Two BamHI restriction sites within Exon4 have been marked with arrows.

During the analysis of cosmid sequences by restriction digestion with various enzymes, to our best luck, the preliminary sequence of the mouse genome was released at the Ensembl web site (www.ensembl.org). The genomic sequence data included whole stonin2 locus with negligible sequence gaps. So we have made use of it in constructing the stonin2 targeting vector.

The $7.5 \mathrm{~kb}$ and $2.5 \mathrm{~kb}$ genomic DNA fragments that hybridized with probe2 (Figure 2.6) were cloned into BamHI digested pUC18 cloning vector. (Figure 2.7) and their orientation and sequences were proven by DNA sequencing. We used a pBS.SKNEO $2 \mathrm{Kpnl}$ vector as the scaffold for the targeting vector. 


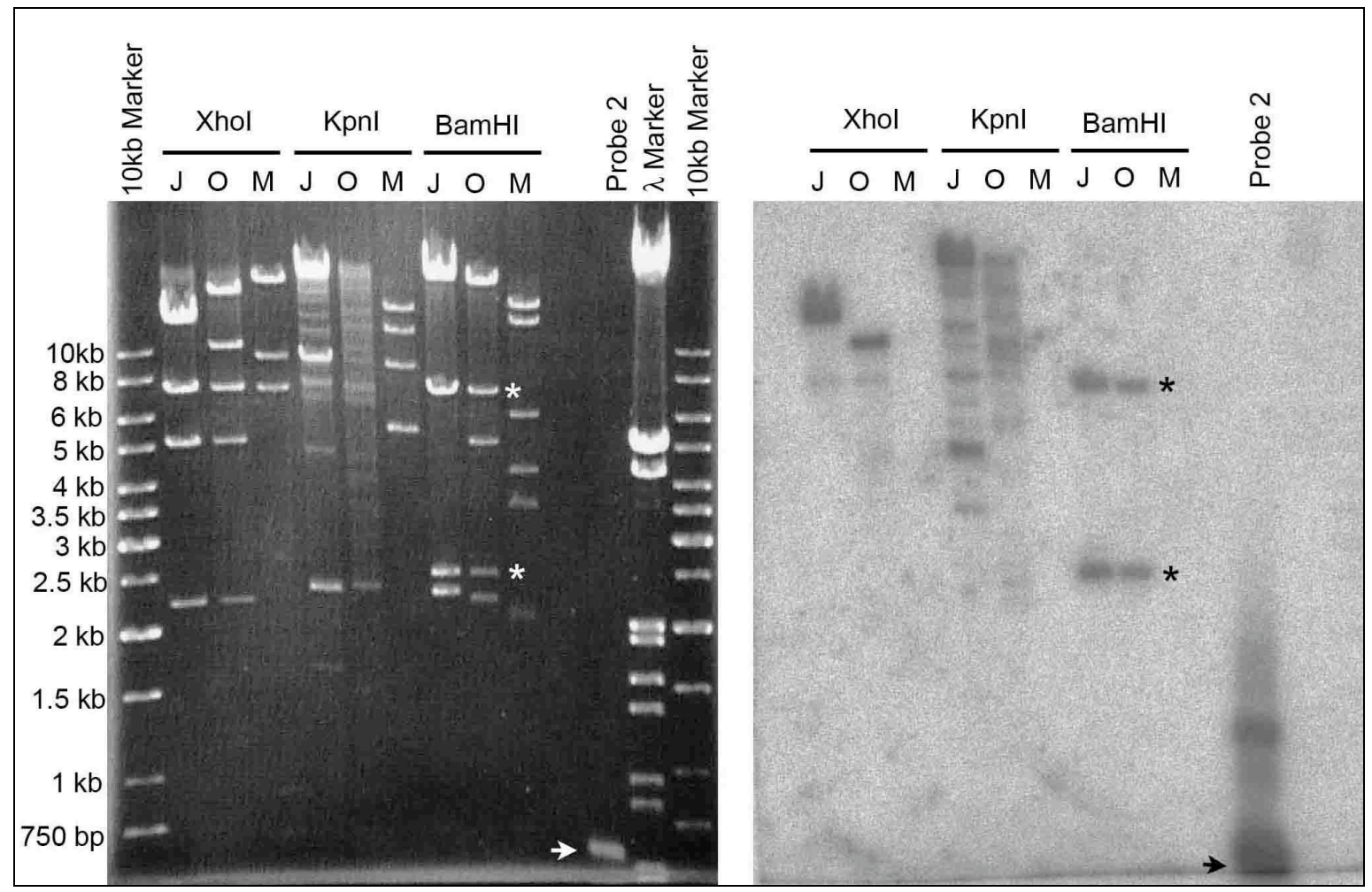

Figure 2.6 Confirmation of cosmid clones by Southern hybridization. $0.5 \mu \mathrm{g}$ of cosmid DNA was restriction digested with the indicated enzymes and separated by a $0.7 \%$ agarose gel. After Southern blotting, probe 2 was used for hybridization. Probe2 was a PCR amplified fragment obtained by primers Pr-2 and Pr-10. It corresponds to the region between BamHI sites (Figure 2.5) and about $100 \mathrm{bp}$ long extra stretch in both directions. Asterices show 2.5 and $7.5 \mathrm{~kb}$ fragments obtained by BamHI digestion that are detected by probe2. Arrows show $50 \mathrm{ng}$ of probe 2 loaded as a positive control.

A $1.6 \mathrm{~kb}$ Xba1 fragment flanking the $4^{\text {th }}$ coding exon of the mouse stonin2 gene at the $5^{\prime}$ end was subcloned into this vector using an Xba1 restriction site upstream of the neomycin cassette. Orientation of the insert was confirmed by restriction digestion and sequencing. Next, a $7.3 \mathrm{~kb}$ fragment from the 3 s side of the $4^{\text {th }}$ coding exon was directionally subcloned cloned to the 3 ' end of the neomycin cassette using HinDIII and Xho1 restriction sites. Cloning was confirmed via restriction digestion and sequencing. This vector called pBS.NEO.XX hereafter, was used for electroporation. In order to generate a shorter alternative construct pBS.NEO.XX was digested with Apa1 and religated. This treatment excises a $3.9 \mathrm{~kb}$ Apa1 fragment from the 3' arm of the vector. This vector is called pBS.NEO.XA and was also used for electroporation. In brief, both vectors contain $1.6 \mathrm{~kb}$ genomic sequence as 5 ' targeting arm. In addition they contain 7.3 or $2.4 \mathrm{~kb}$ sequence as 3 ' targeting arm. Upon homologous recombination, $1.3 \mathrm{~kb}$ of coding sequence from exon 4 is replaced with the $1.2 \mathrm{~kb}$ neomycin cassette (Figure 2.8). 


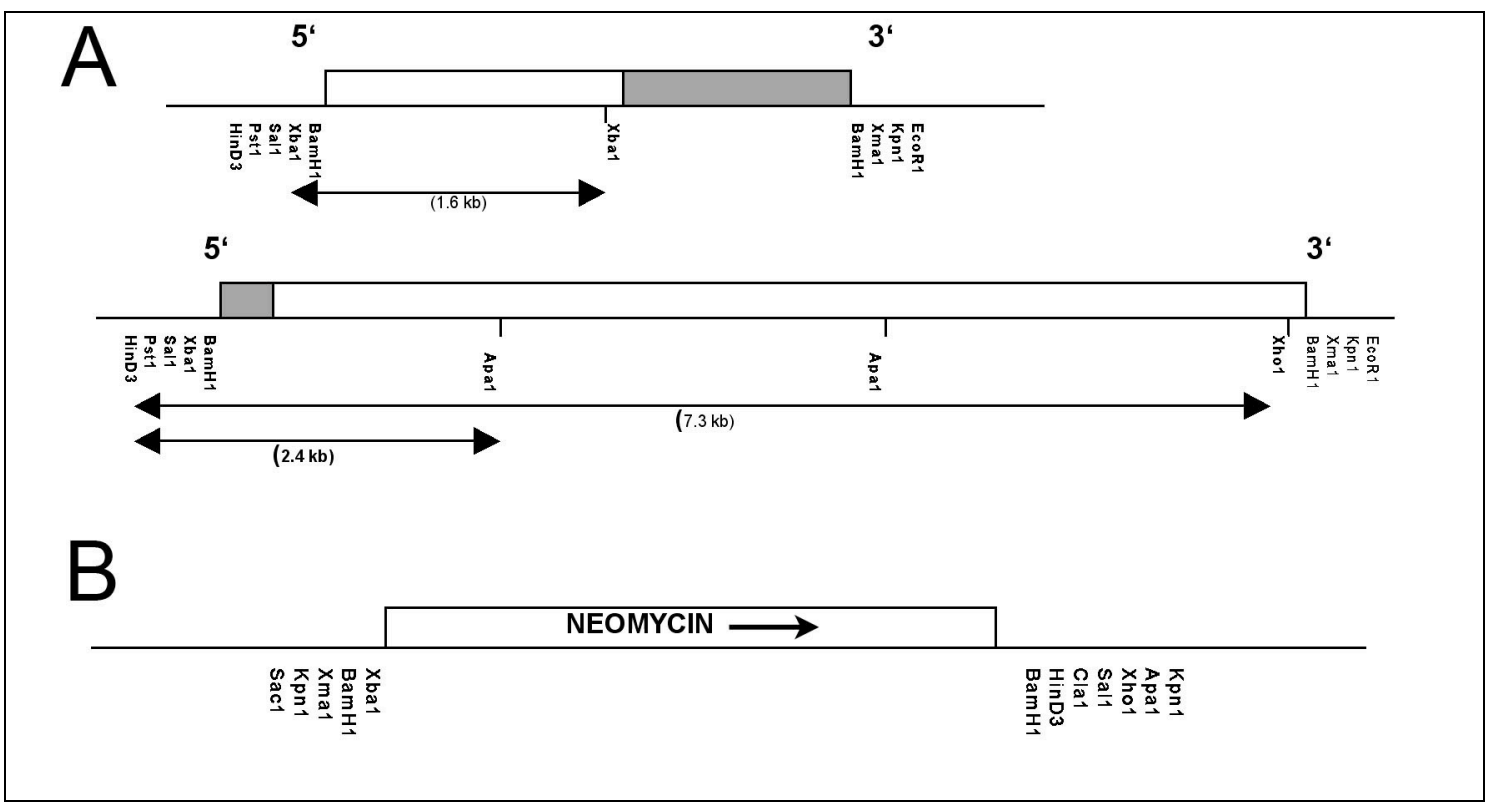

Figure 2.7 Cloning of the targeting construct. (A) Cosmid O DNA was digested with BamH1, Southern blotted and hybridized with a probe within exon 4 sequence. Two fragments of $7.5 \mathrm{~kb}$ and $2.5 \mathrm{~kb}$ hybridized to this probe. These two fragments were cloned into BamHI digested pUC18 vector and the ends were sequenced. Their orientations in the pUC18 vector are shown in the figure. Gray areas correspond to exon DNA. The fragments used for the targeting construct are shown by double sided arrows. 5' and 3' markations indicate orientation of stonin2 gene. (B) . pBS.SK-NEO*2Kpn1 vector and its multiple cloning site. Neomycin cassette is under the control of a pTK promoter and contains a poly adenylation site. Arrow shows direction of transcription.

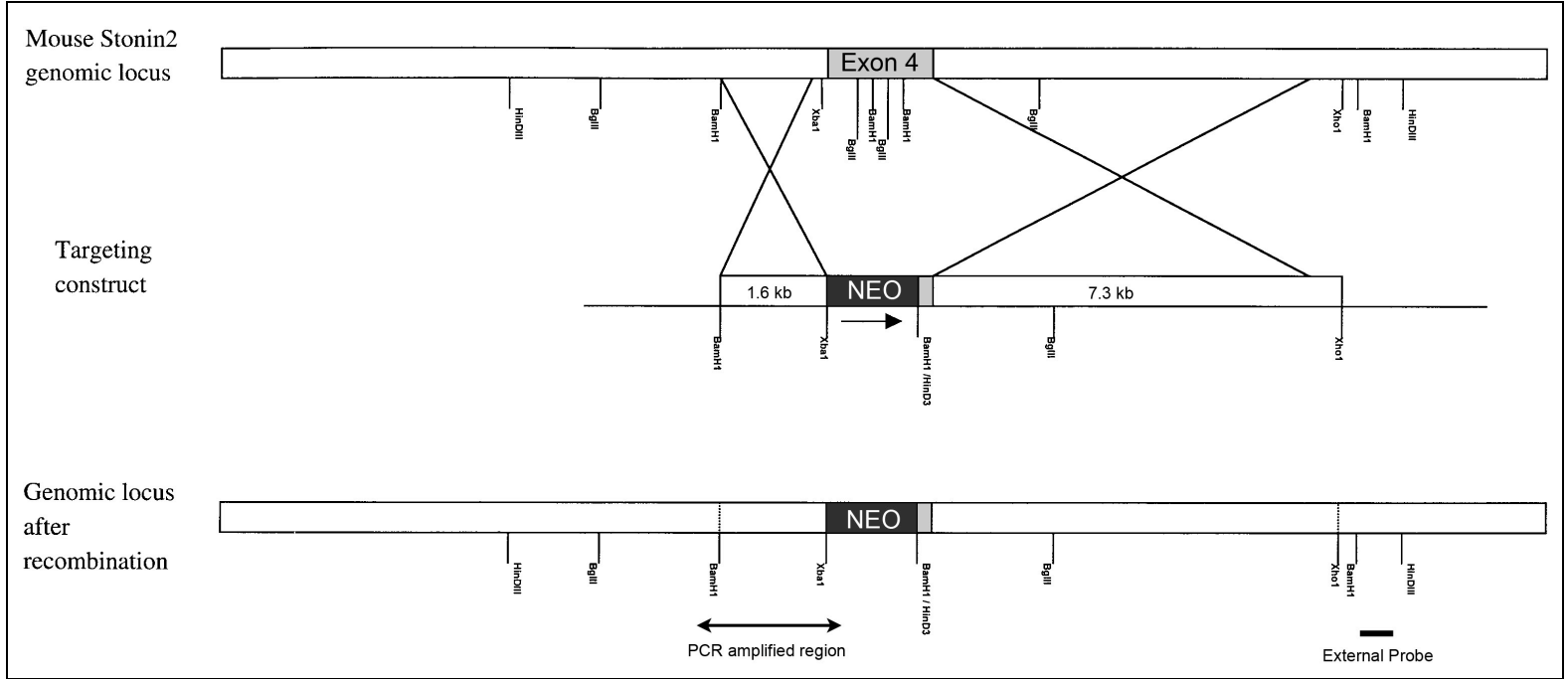

Figure 2.8 Targeting strategy for mouse stonin 2 genomic locus. Stonin 2 exon 4 sequence is partially deleted upon homologous recombination and insertion of neomycin cassette. A HinDIII restriction site is introduced to 3' side of the neomycin cassette which was used for screening later. Locations of the external probe for southern hybridization and PCR amplified region during PCR based screening procedure were also shown in the figure. The targeting construct was linearized by Xhol restriction digestion.

\subsubsection{Screening of embryonic stem cell colonies and microinjection}

After electroporation of the targeting construct, many ES cell colonies gained resistance against geneticin but only few of these colonies had actually undergone 
homologous recombination. Southern hybridization and PCR dependent strategies have been used for screening of homologous recombination events in selected ES cell colonies. Both procedures were essential as southern hybridization could test the correct recombination event only at 3 ' side and PCR screening could test only the 5' side of the locus (see Figure 2.8).

\subsubsection{Southern based screening}

For Southern hybridization based screening, it is essential to select an external probe whose sequence matches with the genomic sequence but not with the targeting vector sequence. Initially we selected an external probe from the 5' side of the targeting vector. However, this probe recognized a DNA smear rather than a single band (data not shown). Closer inspection of the probe sequence by BLAST searches in mouse genome DNA database showed that this probe contained sequence elements that were repeated widely in the genome with sequence conservation approaching $90 \%$. Therefore, we thrusted this probe aside and selected another external probe from the 3 ' side of the targeting vector (Figure 2.8). This probe was amplified using primers $\operatorname{Pr}-18$ and $\operatorname{Pr}-19$ and it recognizes a single DNA band of 15.5 kb size from HinDIII digested WT genomic DNA (Figure 2.9).

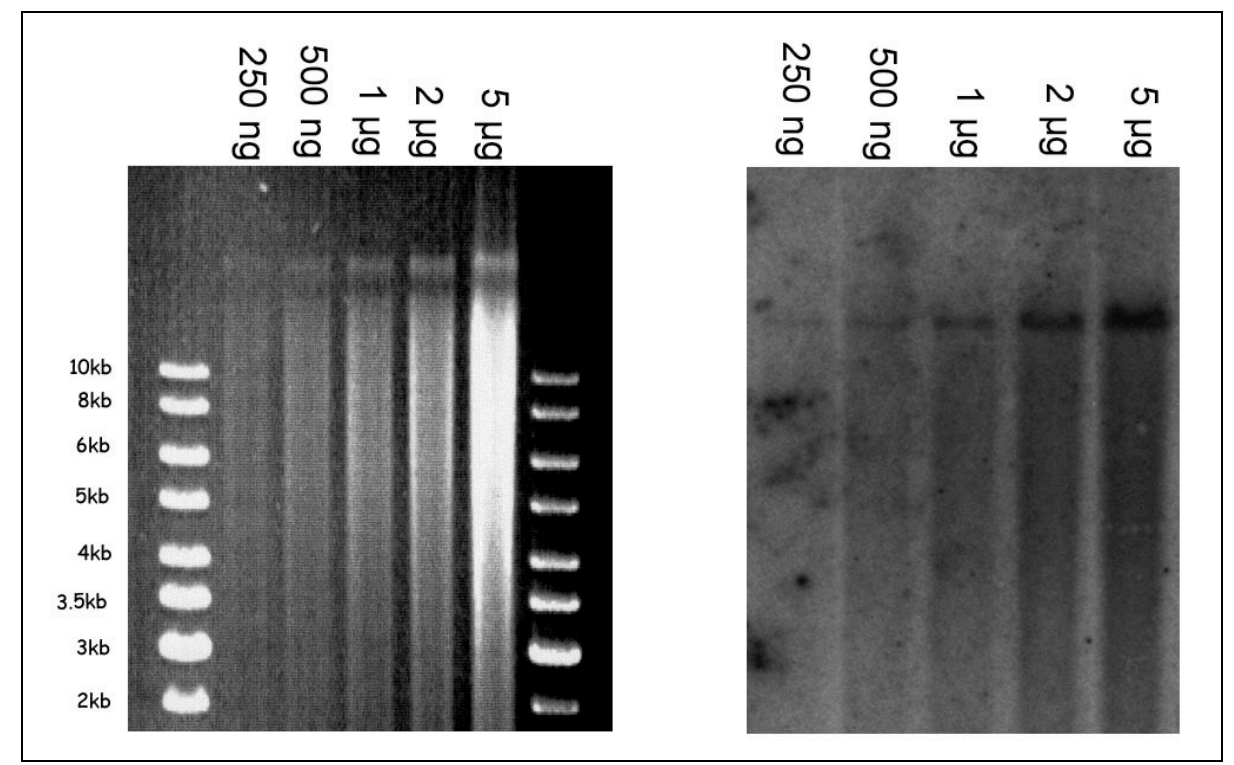

Figure 2.9 Testing of the external probe. Different quantities of genomic DNA from WT embryonic stem cells was restriction digested with HinDIII enzyme, separated by gel electrophoresis and Southern blotted. Upon hybridization with the external probe amplified using P-18/P-19 primers, a single DNA fragment of $15.5 \mathrm{~kb}$ size was detected as expected.

Upon homologous recombination, a HinDIII restriction site is introduced at 3' side of the neomycin cassette. This would result in recognition of a new $9.2 \mathrm{~kb}$ fragment 
after Southern hybridization in addition to the $15.5 \mathrm{~kb}$ fragment coming from the WT locus. Screening of ES cell genomic DNA obtained from the first 23 clones (plate F) using this procedure resulted in identification of 3 positive clones (Figure 2.10). However, one of these clones, F13, was negative according to PCR based screening. Probably, the 3 ' side of the targeting vector was inserted into the genome by homologous recombination of the longer arm and 5' side was randomly inserted. After PCR based screening procedure was established, the rest of the ES cell colonies were initially screened with PCR, the positive ones were selected and retested by southern hybridization.

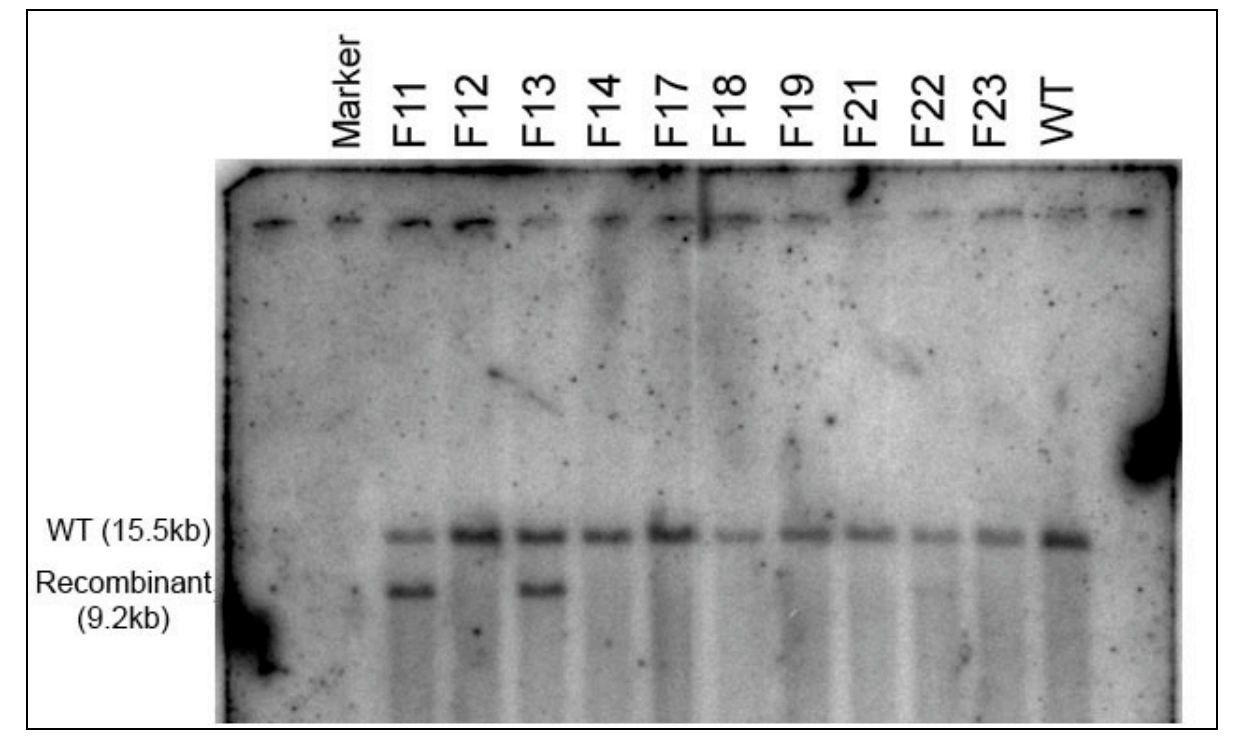

Figure 2.10 Southern screening of ES cell colonies. Genomic DNA samples from the selected colonies were screened by Southern hybridization. F11 and F13 clones have a second band of $9.2 \mathrm{~kb}$ as a result of recombination.

\subsubsection{PCR based screening}

An alternative and easier strategy for screening for positive clones was performed by PCR. In this approach, two primers were selected: one from the neomycin cassette sequence and the other from the genomic sequence that was not included in the targeting vector (Figure 2.8). 5' side of the targeting vector was chosen for PCR screening as it was shorter. Two 5' side primers (up1 and 2) and two 3' side primers ( low1 and 2) have been selected and used as primer pairs (4 pairs). Up1-Low2 primer pair was chosen for further screening as it yielded the best PCR product tested on genomic DNA samples with known genotype according to Southern hybridization. The other primer pairs also yielded the expected size fragments. The following PCR program was used for screening of all ES cell colonies. Taq polymerase was the 
enzyme of choice as it yielded better results. After 25 cycles of PCR, additional Taq polymerase was added and 15 more cycles were performed. A 1910bp DNA fragment was amplified only from colonies that underwent homologous recombination at the $5^{\prime}$ arm (Figure 2.11).

$\left.\begin{array}{cll}\hline \multicolumn{2}{c}{\text { ESC screen PCR cycle }} \\ \hline 95^{\circ} \mathrm{C} & 10 \mathrm{~min} & \text { Initial denaturation } \\ 95^{\circ} \mathrm{C} & 30 \mathrm{sec} & \text { Denaturation } \\ 60^{\circ} \mathrm{C} & 45 \mathrm{sec} & \text { Annealing } \\ 72^{\circ} \mathrm{C} & 3 \mathrm{~min} & \text { Extension } \\ 72^{\circ} \mathrm{C} & 5 \mathrm{~min} & \text { Final extension } \\ 4^{\circ} \mathrm{C} & \infty & \text { Storage }\end{array}\right\} 25 \mathrm{X}+15 \mathrm{X}$

As PCR is a contamination sensitive procedure and the risk of contamination of negative colonies with cells from positive colonies is high during the colony picking procedure, the PCR results were not regarded as entirely trustable and the colonies were further checked by Southern hybridization. As a result of screening by PCR and Southern hybridization 4 positive clones have been identified out of 149 picked up colonies: F9, F11, A15 and C15.

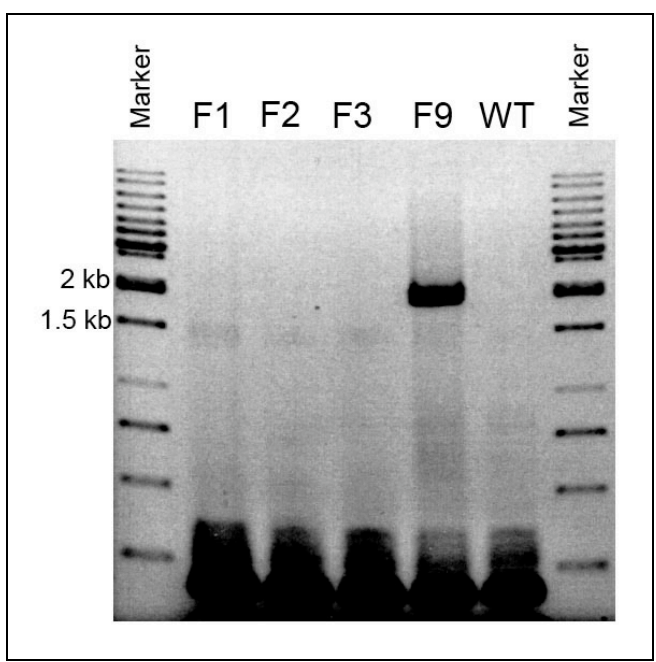

Figure 2.11 PCR based screening of ES cell colonies. 50ng of genomic DNA from the indicated ES cell colonies was used as a template for PCR amplification. A $1.9 \mathrm{~kb}$ fragment is amplified only from clone F9, which was tested to be positive before by Southern hybridization (data not shown).

\subsubsection{Microinjection of positive ES cell colonies and generation of chimera}

Frozen vials of $\mathrm{A} 15$ and $\mathrm{F} 11$ clones were thawed and expanded for 5 days on PMEF covered, recombinant LIF (ESGRO) supplied culture dishes. No signs of differentiation were eminent during the culturing period. The identity of both clones was reconfirmed via southern blotting and PCR before microinjection. Aliquots of ES 
cells were taken and stored in liquid $\mathrm{N}_{2}$ for possible future microinjections. ES cells from clones F11 and A15 were microinjected into blastocyts from C57Black6/N mice by Monika Schindler (Animal Facility of MPI Experimental Medicine, Göttingen) and these chimeric blastocyts were implanted to pseudopregnant mice. The microinjection dates were 20/21 January 2003 respectively. We have obtained 4 male chimeras from clone $A 15$ ( $7^{\text {th }}$ of February 2003) while clone F11 did not yield chimeric animals. The degree of chimerism was estimated to be between $30 \%$ and $90 \%$. One of the four chimeras was eaten by his mother before reaching maturity. The remaining 3 chimeras were transported to animal facility of Biochemistry 2 . They were mated with C57Black6 female mice for the establishment of a mouse colony.

A second round of microinjection was performed on $19^{\text {th }}$ and $20^{\text {th }}$ of February 2003. ES cells from clone F11 (refrozen vial) and C15 were expanded in the same way. Out of these microinjections we obtained 9 chimeras on 9 / 10 March 2003 (8 from F11 and 1 from C15). Some of these animals have also been used in generation of stonin2 colony.

\subsubsection{Immunocytochemistry}

Immunofluorescence detection of exogenously or endogenously expressed proteins was a commonly used procedure in this study. Cells grown on poly-L-lysine (Sigma) or Matrigel (BD Biosciences) coated cover-glasses were briefly washed twice with PBS. Cells were then fixed for $30 \mathrm{~min}$ at RT (15 min for neurons) using 4\% (w/v) PFA in PBS. When required, cells were fixed in $-20^{\circ} \mathrm{C}$ methanol for $6-8$ minutes instead of PFA. After fixation cells were washed 2 times with PBS and sometimes they were stored in PBS at $4^{\circ} \mathrm{C}$ overnight before immunostaining. Cells were permeabilized in GSDB for 15 min and incubated with primary mouse or rabbit antibodies diluted in GSDB (or GSDB-light for some antibodies) for $2 \mathrm{~h}$ at RT. Then they were washed once in high salt PBS and 3 times in PBS for 10 min each. Finally, cells were incubated with goat secondary antibodies diluted in GSDB for $1 \mathrm{~h}$ and washed 3 times in PBS as before. Vectashield (Linaris) or Immumount (Thermo) mounting reagents were used for mounting of the cover-glasses over slides. The slides were stored in a dark slide chamber at $4^{\circ} \mathrm{C}$.

\subsubsection{Preparation of cell and tissue extracts}


For preparation of cellular protein extracts, cells that were grown on plastic culture dishes were washed 2 times with ice cold PBS and scraped using a rubber policeman in PBS. Cells were pelleted by spinning down at $200 \mathrm{~g}$ for $3 \mathrm{~min}$ in a conical tube and washed once with PBS. The cell pellets were resuspended in ice cold IP buffer without triton (20mM HEPES-NaOH pH 7.4, $150 \mathrm{mM} \mathrm{NaCl,} 2 \mathrm{mM} \mathrm{MgCl}_{2}$, $1 \mathrm{mM}$ PMSF, $1 \%$ triton $\mathrm{X}-100)$ by pipetting. Cells were lysed by the addition of triton up to $1 \%$ and pipetting gently $10-20$ times and incubating on ice for $30 \mathrm{~min}$. Cell lysates were cleared by spinning down at maximum speed for $15 \mathrm{~min}$ in a table-top centrifuge. The supernatants were collected and the protein concentrations were measured by Bradford assay.

Rat and mouse brain extracts and other tissue extracts were prepared in a similar fashion. Frozen tissue pieces were plunged into the glass homogenization cylinder filled with 5-10 $\mathrm{ml}$ of homogenization buffer (4mM HEPES-NaOH pH 7.4, 320mM sucrose, 1mM PMSF). Tissue pieces were homogenized using a teflon pestle and applying 10-15 strokes at 900rpm rotation speed. The homogenates were poured into a glass tube and spinned down at $1000 \mathrm{~g}$ for $10 \mathrm{~min}$. The supernatant fractions were promptly collected and up to $1 \%$ triton was added for solubilization and extraction of proteins. Extracts were incubated on ice for $30 \mathrm{~min}$ and occasionally vortexed to enhance solubilization. When performing colP experiments, vortexing was avoided and extracts were mixed by gently swirling the tubes. Insoluble particles were cleared by spinning down the extracts for 30 min using Sorvall SS34 rotor (18000rpm or $25000 \mathrm{~g}$ average) or an equivalent rotor. The extracts were spinned down again for 20 min using a Beckman Optima TLX ultra centrifuge and TLA 100.1 rotor at $70000 \mathrm{rpm}$ speed. After the centrifugations, extracts were assumed to be free of insoluble particles. The protein concentrations were measured and the extracts were kept on ice not longer than a couple of hours before they were used in biochemical experiments.

\subsubsection{Primary culture of neurons and glial cells}

Protocols derived from Banker and Goslin (1998) were applied for culturing of neurons and glial cells. Hippocampal or cortical neurons were prepared from neonatal Day 0,1 or 2 pups of rats or mice. Pups were decapitated and brains were carefully removed and plunged into a petri dish filled with ice cold Hank's salt solution $($ Sigma $)+20 \%$ FCS. Hippocampus or cortical regions were isolated by dissection 
under a Olympus stereo microscope and transferred to a fresh petri dish containing Hank's solution and FCS as before. Hippocampus was chopped into fine pieces and transferred to a $15 \mathrm{ml}$ conical tube. Tissue pieces were washed 2 times with Hank's solution+20\% FCS and 3 times with Hank's solution only. For digestion, tissue pieces were incubated at $37^{\circ} \mathrm{C}$ for $10 \mathrm{~min}$ in $2 \mathrm{ml}$ digestion solution $(137 \mathrm{mM} \mathrm{NaCl}, 5 \mathrm{mM} \mathrm{KCl}$, $7 \mathrm{mM} \mathrm{Na}_{2} \mathrm{PO}_{4}, 25 \mathrm{mM}$ HEPES-NaOH pH 7.2) containing $10 \mathrm{mg}$ Trypsin and $1 \mathrm{mg}$ DNAsel (Sigma). Digested tissue was washed 2 times with $10 \mathrm{ml}$ Hank's solution+20\% FCS and 3 times with Hank's solution as before. For dissociation, tissue pieces were triturated 5-10 times with siliconized pasteur pipettes in $2 \mathrm{ml}$ dissociation solution containing $1 \mathrm{mg}$ DNAsel. 3ml of Hank's solution+20\%FCS was added and dissociated cells were spinned down at $200 \mathrm{~g}$ for $10 \mathrm{~min}$. Supernatant was removed and pellet was resuspended with a siliconized pasteur pipette in $2 \mathrm{ml}$ plating-medium ( MEM(Gibco), $0.5 \mathrm{mg} / \mathrm{ml}$ glucose, $200 \mu \mathrm{g} / \mathrm{ml} \mathrm{NaHCO} 3,50 \mu \mathrm{g} / \mathrm{ml}$ transferrin, $10 \%$ FCS, $2 \mathrm{mM} \mathrm{L-glutamine,} 2.5 \mu \mathrm{g} / \mathrm{ml}$ insulin). Cells were counted by a Neubauer chamber, and 50.000-100.000 cells were seeded on matrigel coated $18 \mathrm{~mm}$ round cover-slips in $1 \mathrm{ml}$ plating medium. $24 \mathrm{~h}$ after plating, $1 \mathrm{ml}$ growth medium was added (MEM, $0.5 \mathrm{mg} / \mathrm{ml}$ glucose, $200 \mu \mathrm{g} / \mathrm{ml} \mathrm{NaHCO} 3,50 \mu \mathrm{g} / \mathrm{ml}$

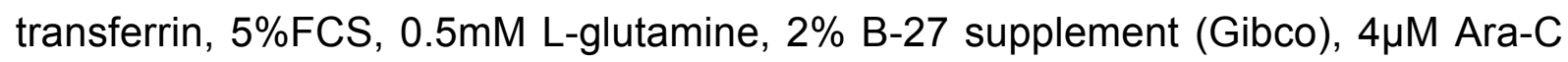
(Sigma)). 4 days after plating another $1 \mathrm{ml}$ of growth medium with $2 \mu \mathrm{M}$ Ara-C was added and cells were kept in the incubator until used.

\subsubsection{Eukaryotic cell culture, transfections and creation of stable cell lines}

\subsubsection{Eukaryotic cell culture}

Eukaryotic cell lines used in this study were explained in section 2.1.10.2. All cell lines were cultured in humidified incubators supplied with $5 \% \mathrm{CO}_{2}$ at $37^{\circ} \mathrm{C}$. Cells were passaged every 3-7 days to a new culture dish and plated 1:5-1:20 dilutions. HEK293, N1E-115, U343 and COS-7 cells were culture in DMEM 4.5g/l glucose supplemented with $10 \%$ heat inactivated FCS and antibiotics (100 units/ml penicillin and $100 \mu \mathrm{g} / \mathrm{ml}$ streptomycin). Same medium with $10 \%$ horse serum and $5 \%$ FCS was used for culturing of PC12 cells

\subsubsection{Transfection of plasmid vectors and siRNAs}

Cells were transfected with plasmid vectors using lipofectamine 2000 reagent when they were $80-90 \%$ confluent and still in growth phase. Cells were transfected with DNA/lipofectamine complexes (prepared according to the manufacturers instructions) 
in serum and antibiotic free medium (generally Optimem was used) for 3-4 h. At the end of this period, cells were either directly seeded on coated cover-glasses or grown overnight in full medium with serum. Sometimes confluent cells growing on coverglasses were transfected. In this case, less transfection reagent was used to decrease toxicity and the cells were transfected overnight in full medium.

siRNA transfections were carried out twice using Oligofectamine or Lipofectamine 2000 reagents (Gibco). Confluent cells growing in 6-well plates were transfected with $3 \mu \mathrm{l}$ of $100 \mu \mathrm{M}$ siRNA duplexes complexed with $3 \mu$ l transfection reagent in serum free medium as before. Cells were replated at 1:5 dilution and grown 48 hours before the next transfection in the same way. $72 \mathrm{~h}$ after the first transfection cells were seeded on cover-glasses and analyzed next day. In case of double transfections with plasmid vectors and siRNAs, always lipofectamine 2000 reagent was used during the first transfection.

\subsubsection{Creation of stable cell lines}

HEK293 cells stably expressing pcFLAG-synaptotagmin1, pcHA-stonin 1, pcHAstonin 2 and pEGFP-stonin 1 were created. All of these vectors contain a neomycin cassette that confers resistance to geneticin, analogous to ES cell selection procedure.

Cells in 6 well plates were transfected with $1 \mu \mathrm{g}$ of the respective constructs. Next day cells were transferred to a $10 \mathrm{~cm}$ dish and cultured in presence of $1000 \mu \mathrm{g} / \mathrm{ml}$ geneticin (Gibco) for 7-8 days until they reached confluence. All of the control cells which were not transfected had died at the end of this period. Cells were seeded again at 1:10 dilution and cultured in selective medium until they reached confluence. For selection of single clones, cells were dissociated by trypsinization and cell density was counted. Cells were diluted and resuspended in such a way that cell density would be 1 cell per $100 \mu l .100 \mu l$ of the resuspension was seeded into the wells of 96-well plate and cells were cultured in selective medium for 10-20 days until they became confluent. 12-24 putative clones were chosen and transferred to 24-well plates for expanding. Expression levels of transgenic constructs were tested by immunofluorescence microscopy. Clones having mild expression levels in all cells were selected and expanded. These clones were stored frozen in liquid $\mathrm{N}_{2}$. 
HEK293 stable cell line expressing lumenal flag tagged synaptotagmin 1 was obtained by expansion of clone 2 and it was called as HEK293-FLAG-Syt1. These cells had roughly equal levels of synaptotagmin 1 stranded on their plasmalemma until passage 10 of the frozen stocks in non-selective medium. After 10 passages some cells ceased to express synaptotagmin1. Whether this can be prevented or delayed by culturing in selective medium has not been tested.

\subsubsection{Antibody internalization assays and quantification}

HEK293-FLAG-syt1 cells were incubated on ice with 1:500 dilution (roughly10 $\mu \mathrm{g} / \mathrm{ml}$ ) of $\alpha-F L A G$ antibody in Optimem for 30 min for surface labeling. Cells were warmed up to $20^{\circ} \mathrm{C}$ or $37^{\circ} \mathrm{C}$ and incubated for the indicated time periods to allow for endocytotic internalization of ligands. Cells were washed twice with PBS and fixed with 4\% PFA in PBS for $30 \mathrm{~min}$ at RT. Primary antibodies bound to non-internalized surface-stranded synaptotagmin 1 were blocked by incubating the cells with goatanti-mouse IgG (1:5 dilution) for $2 \mathrm{~h}$ at RT in GSDB without triton. Cells were washed once with PBS and permeabilized in regular GSDB and stained with primary and secondary antibodies (diluted in GSDB) as explained before. Cell nuclei were stained by DAPI which was included in the mounting reagent.

For quantification of antibody internalization levels, 3 independent experiments were carried out for each experimental condition. Slidebook 4.0.10 software (Intelligent Imaging Innovations Inc.) was utilized for quantifying the results. Using equal exposure times, 3 fluorescence images were acquired from each experiment giving 9 data sets in total. Each data set contained 10-30 cells (transfected or untransfected depending on the experiment). The fluorescence intensity of the internalized antibody was calculated by subtracting the background fluorescence from the total fluorescence intensity. For experiments using different stonin 2 constructs, the fluorescence intensity was measured only from the transfected cells. The fluorescence intensity of any particular data set represents the average intensity of the cells in that data set. Error bars in the figures depict the standard deviation of values obtained from 9 data sets.

\subsubsection{Mouse colony management}

Stonin 2 mouse colony was maintained in conventional accomodations. They were kept in rooms that were continuously air conditioned and monitored for temperature and humidity. $12 \mathrm{~h}$ light/dark cycles were applied to imitate day and night. 
For the initial establishment of the colony, chimeric male mice obtained from microinjection of F11 and A15 ES cell clones were crossed with WT BL6 females. The agouti colored offsprings were genotyped when they reached 3 weeks age. Heterozygous animals were crossed between each other to generate the stonin 2 knock-out animals. Knock-out and WT animals were crossed with each other (only identical genotypes were crossed) and animals were maintained in a mixed background (50\% 129/ola and 50\% BL6). Whenever necessary, genotyping of animals was performed after they reached 3 weeks age (Figure 2.12). For neuronal preparations, neonatal pups were genotyped on the day of birth. Offsprings were separated from their mothers 3-4 weeks after birth.

Genotyping was carried out by PCR using two primer pairs testing for the presence of either neomycin gene(Neo_Fwd1 and Neo_Rev1) or a WT exonic fragment deleted from the stonin2 knock-out loci (Pr-22 and Pr-23). 10-50ng of genomic DNA was amplified in the following PCR reaction using Taq DNA polymerase:

\begin{tabular}{cll}
\hline \multicolumn{2}{c}{ Genotyping PCR } \\
\hline $95^{\circ} \mathrm{C}$ & $10 \mathrm{~min}$ & Initial denaturation \\
$95^{\circ} \mathrm{C}$ & $30 \mathrm{sec}$ & Denaturation \\
$60^{\circ} \mathrm{C}$ & $30 \mathrm{sec}$ & Annealing \\
$72^{\circ} \mathrm{C}$ & $30 \mathrm{sec}$ & Extension \\
$72^{\circ} \mathrm{C}$ & $2 \mathrm{~min}$ & Final extension \\
$4^{\circ} \mathrm{C}$ & $\infty$ & Storage \\
\hline
\end{tabular}

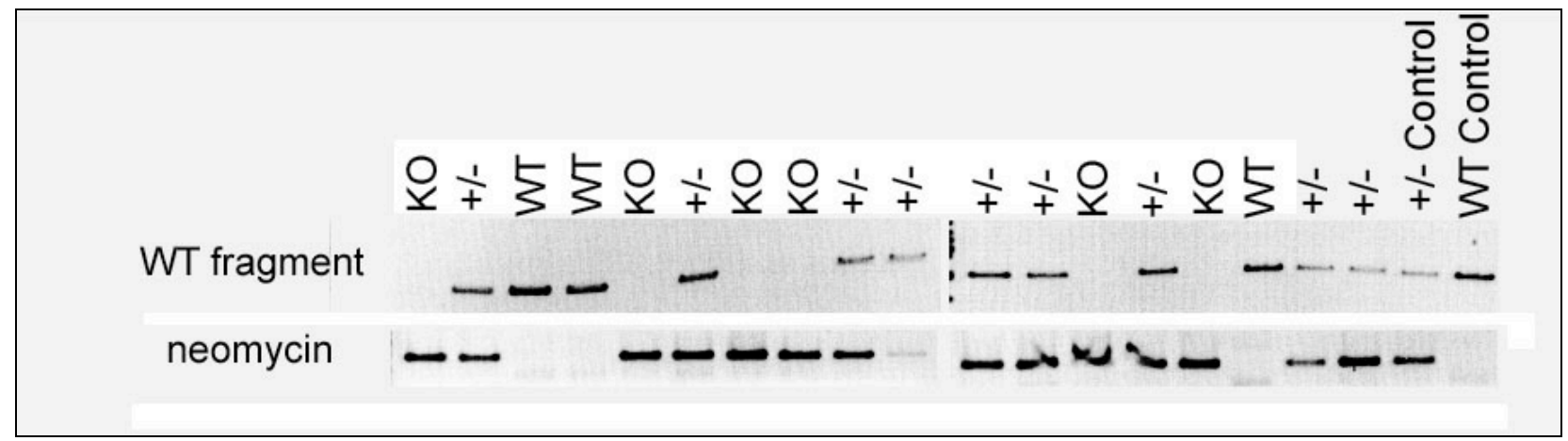

Figure 2.12 Genotyping of 3 week-old pups by PCR. Tail DNA from 3 weeks old pups of heterozygous male and female parents was used in genotyping. KO pups are positive only for the neomycin fragment amplified by neomycin primers whereas the WT pups only for WT fragment amplified by P-22/P-23 primers. Control DNA samples were also amplified during genotyping. 


\section{RESULTS}

\subsection{Tissue distribution and intracellular localization of mammalian stonin 2}

As a first attempt to understand the function of the stonin 2 protein, we examined its distribution of expression pattern in various tissues. To this aim total protein extracts obtained from adult rats were analyzed. Stonin 2 appeared to be exclusively enriched in the brain, but was present at lower levels in all other tissues analyzed (Figure 3.1). In contrast, Hsc-70, a ubiquitous ATPase with chaperone function, was present at roughly equal amounts in all tissues tested. Interestingly, a significantly higher expression level for stonin 2 was seen within the lungs. Whether this is due to expression within endohelial cells or dorsal root ganglia that innervates these remains to be determined.

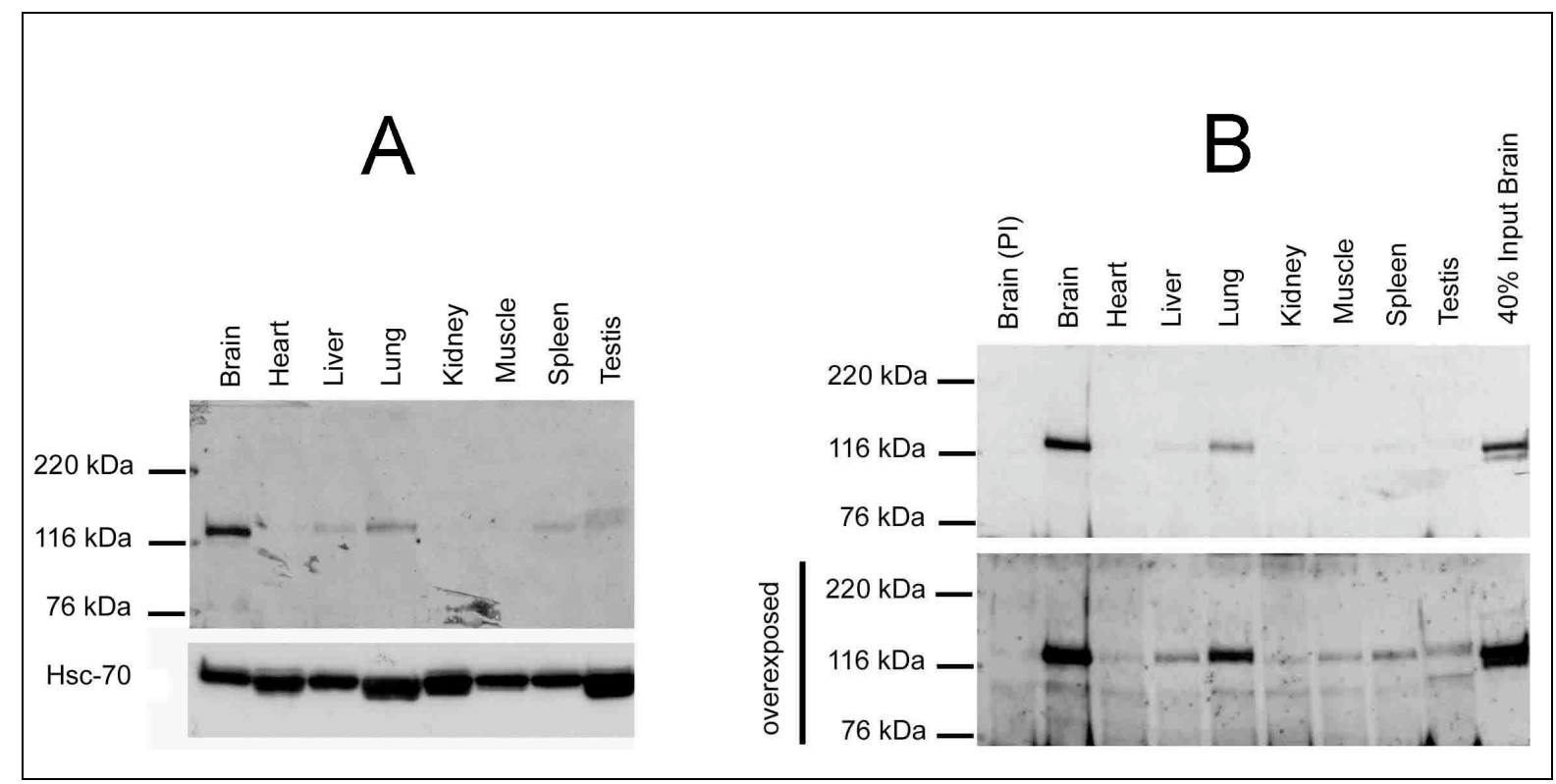

Figure 3.1 Tissue distribution of stonin 2 protein. (A) Western blot analysis of equal amounts of $(40 \mu \mathrm{g})$ protein extracts from the indicated tissues. Stonin 2 runs as a $120-130$ kDa protein in SDSPAGE. (B) Protein extracts from indicated tissues were obtained by boiling tissue homogenates in $2 \%$ SDS containing solution. Prior to IPs using stonin 2 antisera (or preimmune sera, lane1) coupled beads, extracts were $1: 10$ diluted so that SDS concentration drops down to $0.2 \%$ and $1 \%$ final Triton $\mathrm{X}-100$ was added. Each lane contains immunoprecipitated samples from $100 \mu \mathrm{g}$ extract. $40 \mu \mathrm{g}$ brain extract was loaded to the last lane as a standard. Note that stonin 2 antiserum quantitatively immunoprecipitates stonin 2.

In order to more clearly define the regions of the brain in which stonin 2 is expressed, we performed WB analysis from total protein extracts derived from different parts of the brain. According to quantitative WB analysis, stonin 2 seemed to be enriched in the hippocampus at least 3-4 fold compared to other brain regions (Figure 3.2B). In contrast, synaptophysin, a synaptic vesicle protein was present at roughly equal 
amounts in all brain areas. In addition to the indicated regions, stonin 2 was also present at similar levels in spinal cord protein extracts (data not shown). In situ hybridization experiments, in which an RNA probe specific for mouse stonin 2 was used, showed that stonin 2 mRNA is expressed in all parts of the brain with particularly high levels in the hippocampus (Figure 3.2A). As a positive control, we used a probe for GRF, a guanine nucleotide exchange factor, known to be expressed primarily in hippocampus and cortex.

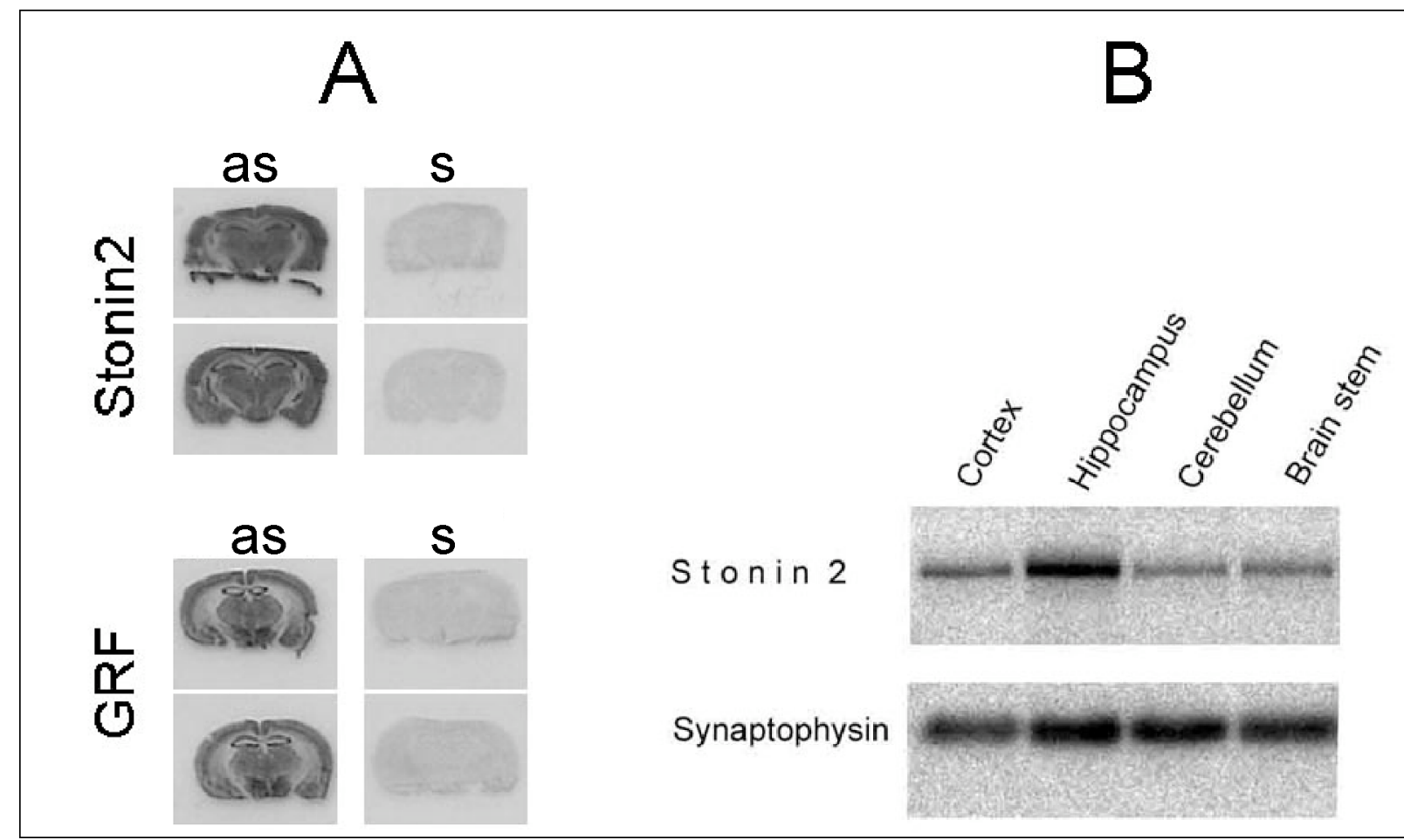

Figure 3.2 Expression of stonin 2 in different brain regions. (A) Coronal brain sections from 6-8 week old mice were in situ hybridized with radioactively labeled antisense (as) or sense (s) RNA probes against stonin 2 or GRF mRNA sequences. Antisense probes against stonin 2 detect an intense stonin 2 expression in the hippocampus. GRF was used as a positive control for hippocampus. (B) The indicated brain regions were obtained by dissection of adult rat brains under a stereo microscope. Equal amounts $(20 \mu \mathrm{g})$ of protein extracts were separated by SDS-PAGE. Protein bands were detected and quantified by the help of $\left[{ }^{125} \mathrm{I}\right]$ labeled protein $\mathrm{A}$.

In addition to tissue extracts, several cell lines or primary cells were tested for stonin 2 expression. We found low levels of stonin 2 expression in N1E neuroblastoma, PC12 neuro-endocrine and INS1E pancreatic islet cell lines. Primary cells from secretory glands such as chromaffin cells and pancreatic islet cells displayed significant levels of stonin 2 expression that were not seen in tumor cell lines derived from those tissues. Fibroblasts such as COS-7, NIH3T3 did not express stonin 2. Interestingly, we found considerable levels of stonin 2 expression in HEK293 (Human 
embryonic kidney) and U343 (human) glioma cells. R1 embryonic stem cells also expressed stonin 2, albeit at somewhat lower levels (Figure 3.3).

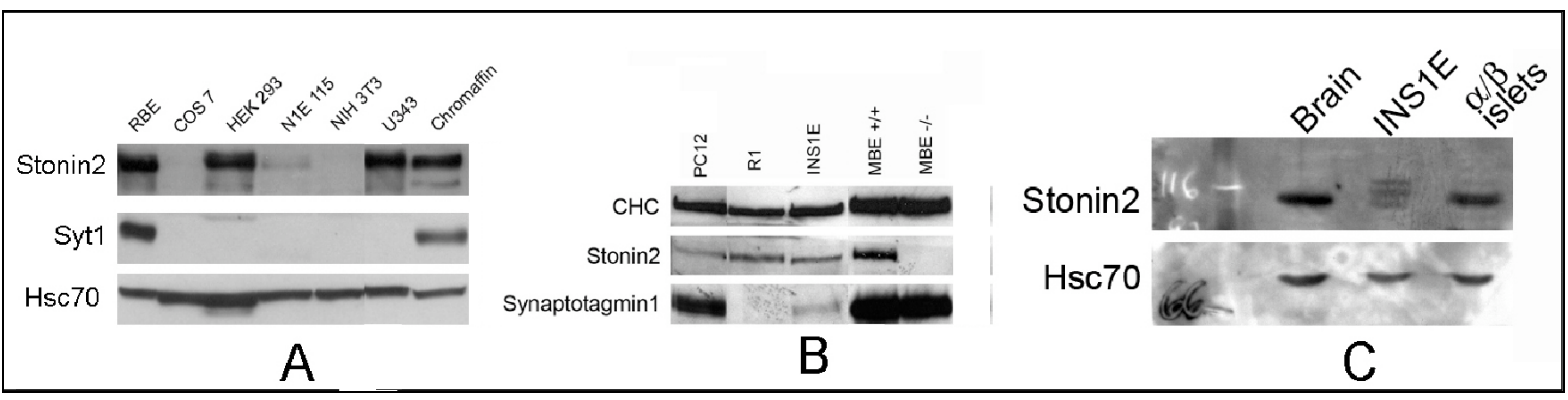

Figure 3.3 Stonin 2 expression in different cell lines and primary cells. Equal amounts of protein extracts obtained from the indicated cell lines or primary cells were separated by SDS-PAGE and probed with stonin 2 and other control antibodies. Experiments were performed at different time points. Brain extracts were loaded as positive controls: RBE (rat brain extract), MBE (mouse brain extract; WT or KO), CHC (Clathrin heavy chain)

Our data somewhat disagree with published findings based on Northern blot analysis, where stonin 2 mRNA has been detected ubiquitously (Martina et al., 2001).

Finally, we analyzed the subcellular distribution of stonin 2 by immunofluorescence microscopy. Double immunostaining of rat brain cortical neurons with antibodies against stonin 2 and synaptotagmin 1 revealed colocalization of both proteins to the synapses (Figure 3.4), consistent with our previous reports (Walther et al., 2001).
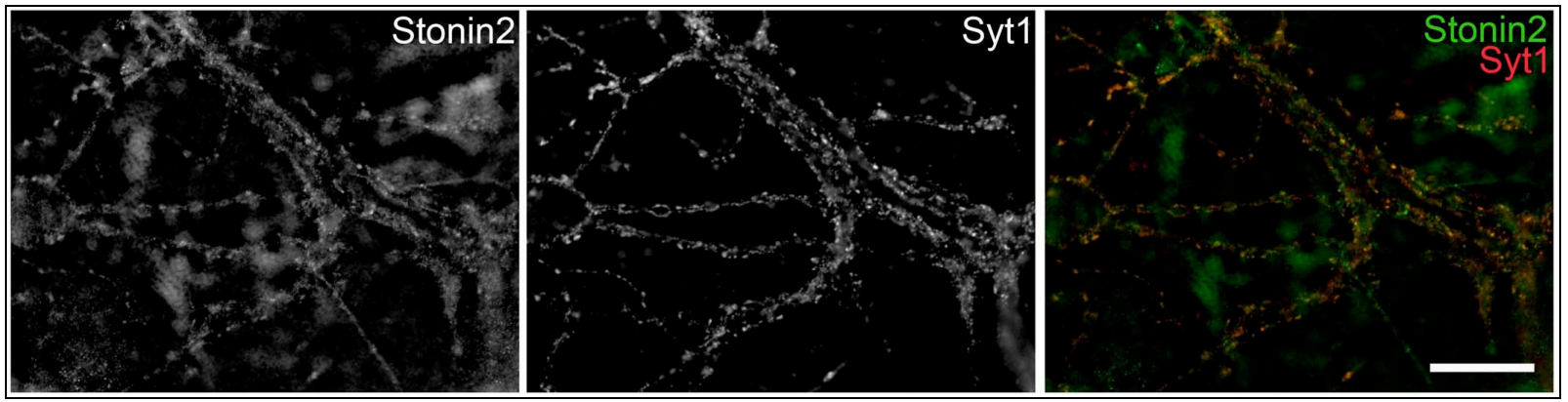

Figure 3.4 Endogenous stonin 2 expression in neurons. Rat brain cortical neurons cultured for three weeks in vitro were fixed and immunostained with antibodies against stonin 2 (green) and synaptotagmin 1 (red) followed by decoration with fluorescently labeled secondary IgGs. Serial images along the z-axis were deconvolved to obtain the images. Scale bar, $20 \mu \mathrm{m}$.

\subsection{Stonin 2-EH domain interactions}

We used a GST-Stonin 2NT(1-555 amino acids) construct to affinity-purify its interaction partners from detergent extracted rat brain lysates (RBE). Unfortunately, extensive degradation of this fusion protein masked any positive interaction partners smaller than $120 \mathrm{kDa}$ from being detected by coomassie blue staining of the SDS gels. Nevertheless, a protein band with an approximate molecular weight of $200 \mathrm{kDa}$ was detected, excised from the gel, and digested with trypsin. Mass spectrometric 
analysis of tryptic fragments identified this protein as the long isofrom of intersectin1 (Figure 3.5A).

In order to asses the role of $\mathrm{Ca}^{2+}$ in Eps15-EH domain and stonin 2-NPF interactions, we performed affinity purification experiments using the amino-terminal domain of stonin 2 fused to GST (Figure 3.5B). Detergent extracted rat brain lysates were incubated with GST-stn2NT or GST in the presence or absence of $\mathrm{Ca}^{2+}$ or a calcium chelator. After washing, bound proteins were analyzed by SDS-PAGE and immunoblotting. Addition of $200 \mu \mathrm{M} \mathrm{Ca}^{2+}$ or chelation of all free $\mathrm{Ca}^{2+}$ in the solution using 2mM EGTA had no apparent effect on the amount of Eps15 or AP2 associated with the GST-stn2NT fusion protein.

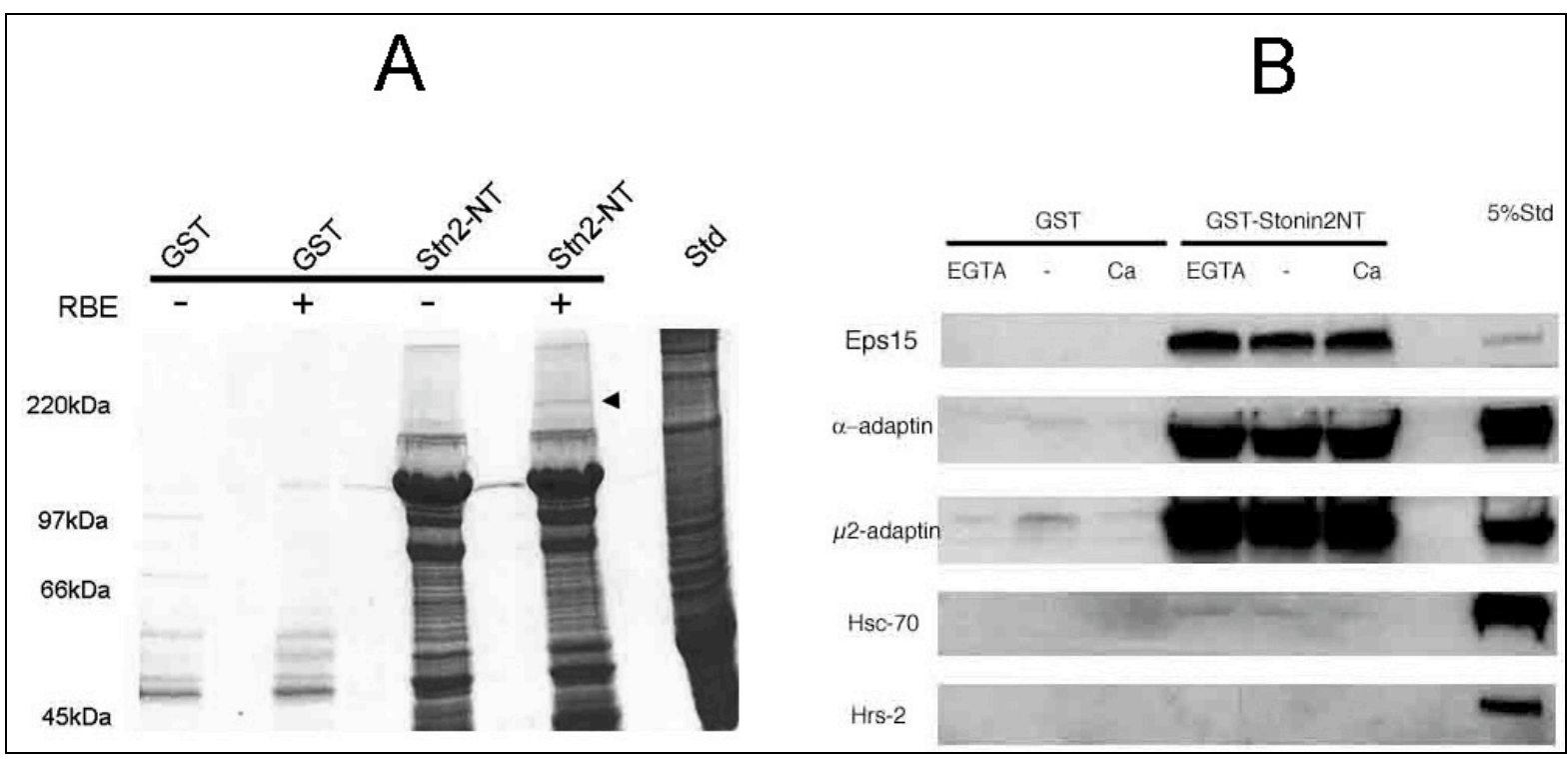

Figure 3.5 Affinity purification of Eps15 and Intersectin1 using GST-Stonin 2NT fusion protein. (A) $3.2 \mu \mathrm{g}$ of RBE were incubated with $80 \mu \mathrm{g}$ of GST or GST-Stonin 2NT coupled glutathione-agarose beads in order to affinity-purify stonin 2 interacting partners. Extensive degradation of purified stonin 2NT masked any possible interacting proteins smalled than $120 \mathrm{kDa}$. The gel was stained with Coomassie brilliant blue solution in order to detect protein bands. The $200 \mathrm{kDa}$ protein shown with an arrowhead was identified as intersectin 1 after mass spectrometry analysis. Standard $100 \mu \mathrm{g}$ (B) $1 \mathrm{mg}$ of RBE was incubated with $30 \mu \mathrm{g}$ of GST or GST-Stonin 2NT bound agarose beads in binding buffer (20mM HEPES pH 7.4, 100mM NaCl, $1 \mathrm{mM}$ PMSF, $1 \mathrm{mM}$ DTT, $2 \mathrm{mM} \mathrm{MgCl}_{2}, 1 \%$ Triton X-100) with $2 \mathrm{mM}$ EGTA or $200 \mu \mathrm{M} \mathrm{Ca}^{2+}$. Bound proteins were eluted by boiling in sample buffer and separated by SDS-PAGE for WB analysis. Note that GST-Stonin 2NT fusion protein quantitatively purifies eps15 from RBE (Compare $5 \%$ std with pull-down lanes).

In order to extend this analysis of stonin 2-EH domain interactions, we cloned all EH domains from mouse eps15 and intersectin1. Affinity-purification experiments were carried out using 6His-tagged $\mathrm{EH}$ domains (thereby avoiding the detection of GSTreactive bands with our antisera) confirmed that EH domain- NPF motif interactions are stronger when higher avidity is achieved by multiple copies of the interaction partners. Highest binding to stonin 2 and epsin 1 was achieved when constructs 
bearing multiple EH domains were used (Figure 3.6). In agreement with published data, we found that multiple EH domains were required for efficient binding to Stonin 2NT fusion proteins.

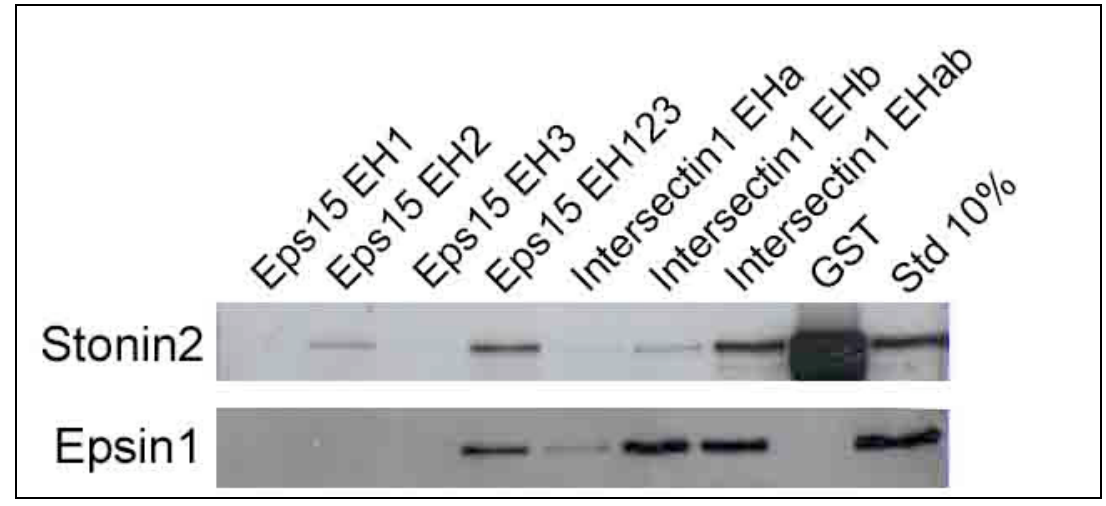

Figure 3.6 Binding of individual EH domains to stonin 2 and epsin 1. $1 \mathrm{mg}$ RBE was incubated with $10 \mu \mathrm{gs}$ of $6 \mathrm{His}$ tagged EH domains coupled to Ni-NTA agarose beads. Highest quantities of epsin 1 and stonin 2 were purified when all EH domains were present. Note that stonin 2 antiserum crossreacts with GST in lane 8.

\subsection{Stonin 2 binds directly to AP2 via a novel type of $\alpha$-ear binding motif}

In our GST pull-down assays using GST-Stonin 2NT as a bait, we were able to purify AP2 subunits from RBE efficiently. However, whether stonin 2-AP2 interaction was direct or indirectly mediated by other proteins such as eps 15 remained unclear. Eps15 contains 15 DPF motifs in its carboxy-terminal part and therefore it may link stonin 2 to the AP2 complex. In order to investigate this possibility, we first made use of the fact that eps 15 can be quantitatively purified by immobilized GST-Stonin 2NT fusion protein and thereby became depleted from the extract. Surprisingly, we could detect similar amounts of AP2 in the eluate if affinity purification was done from eps15 depleted rat brain extracts (Figure 3.7). Although, the presence of unidentified bridging proteins could not be excluded, these data prompted us to investigate whether stonin 2 is able to directly bind to AP2. 


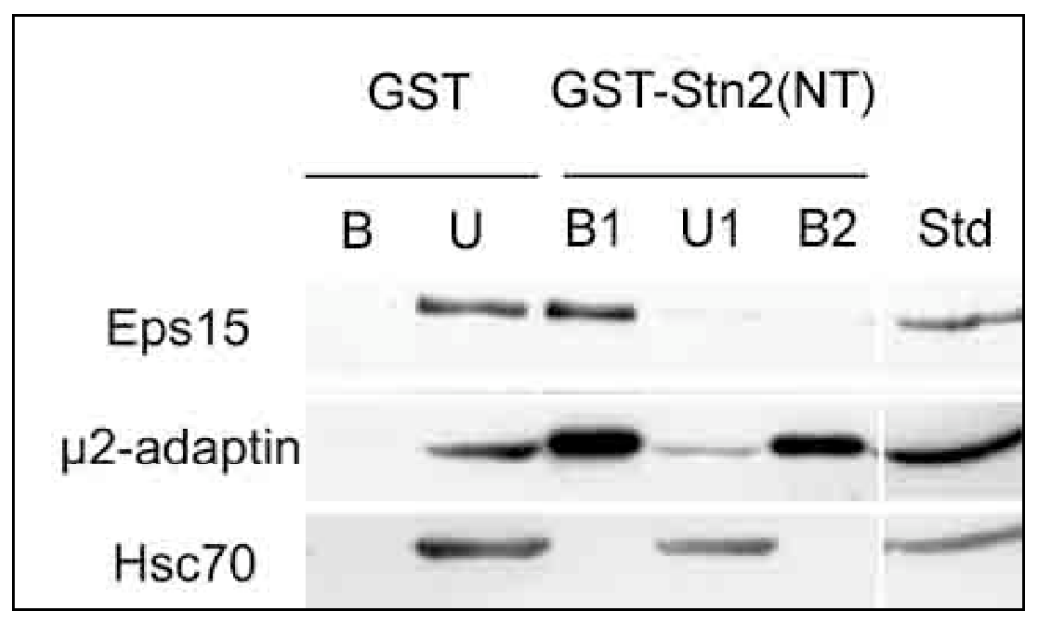

Figure 3.7 Binding of stonin 2 to AP2 in eps15 depleted RBE. RBE was incubated with GST or GST-Stonin 2NT fusion protein bound agarose beads. After the incubation beads were recovered (B1) and extract (U1) was re-incubated with a fresh batch of protein bound beads (B2). B: Bound, U: Unbound.

Many endocytic accessory proteins harbor structurally unfolded linear peptide sequences that are recognized by AP2 subunits or clathrin. DPF motifs within eps15, DPW within epsin 1 and FXDXF type motifs within amphiphysins provide examples for peptide sequences capable of association with the AP2- $\alpha$-appendage domain. The strong interaction between stonin 2 and AP2 suggested the presence of one or likely multiple AP2 interaction motifs within stonin 2. However, analysis of the stonin 2 amino acid sequence did not reveal the presence of any canonical FXDXF or DXF/W boxes .

In order to test if stonin 1 is also able to interact with AP2, we generated GST fusion constructs of full length mouse stonin 1 and its various truncations (serine/proline rich domain: 1-200aa, stonin HD+ $\mu \mathrm{HD}$ : 201-730aa and $\mu \mathrm{HD}$ alone: 403-730aa). Affinity purification experiments from RBE showed that stonin 1 is capable of binding to AP2 via a putative interaction motif present within its amino-terminal first 200 amino acids encompassing the serine/proline rich domain (Figure 3.8). In contrast to stonin 2, we could not detect any significant interaction between stonin 1 and EH-domain containing proteins such as Eps15 or intersectin 1. This is likely due to the fact that stonin 1 contains only a single NPF motif insufficient for high-affinity EH-domain binding. 


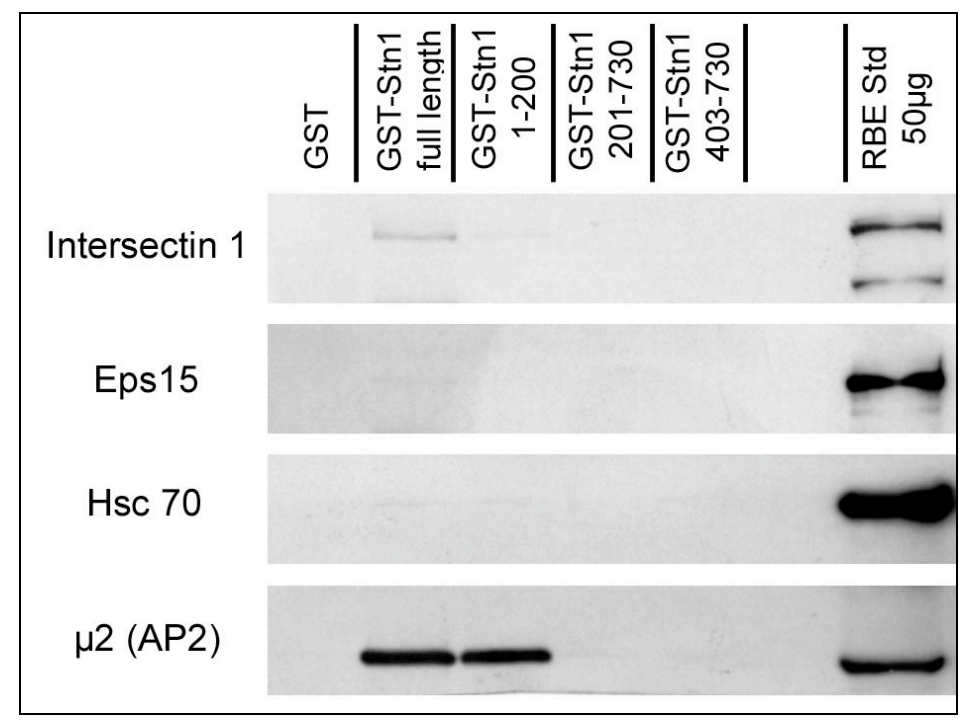

Figure 3.8 Interaction of stonin 1 with AP2, eps15 and intersectin 1. GST or GST-Stonin1 fusion protein bound beads were incubated with $1 \mathrm{mg}$ RBE by gentle mixing on a rotating wheel in order to affinity purify those proteins from RBE that interact with stonin 1 . After three washes, bound proteins were eluted from the beads by boiling in sample buffer. After separation by SDS-PAGE, proteins were blotted to nitrocellulose membrane and decorated with antibodies against indicated proteins.

The amino-terminal serine/proline rich domains of stonin 1 and stonin 2 display little sequence conservation using different peptide sequence alignment algorithms. To reveal putative AP2 interaction motifs, we performed a dot plot alignment of the amino-terminal domains of stonins 1 and 2, searching for short (4-6 amino acids), nearly exact matches ( $>75 \%$ identity). Using these parameters we discovered a putative motif with the consensus sequence WVXFaaa with any of the amino acids being an acidic residue. (Figure 3.9). This motif is present once in stonin 1 and three times in stonin 2. Database searches for proteins harboring similar peptide sequences yielded several other endocytic accessory proteins including synaptojanin, pacsin/syndapin, and intersectin 2 . In some of these cases, AP2 interaction was known but no interaction motif had been identified (Conner et al., 2002; Haffner et al., 2000). 


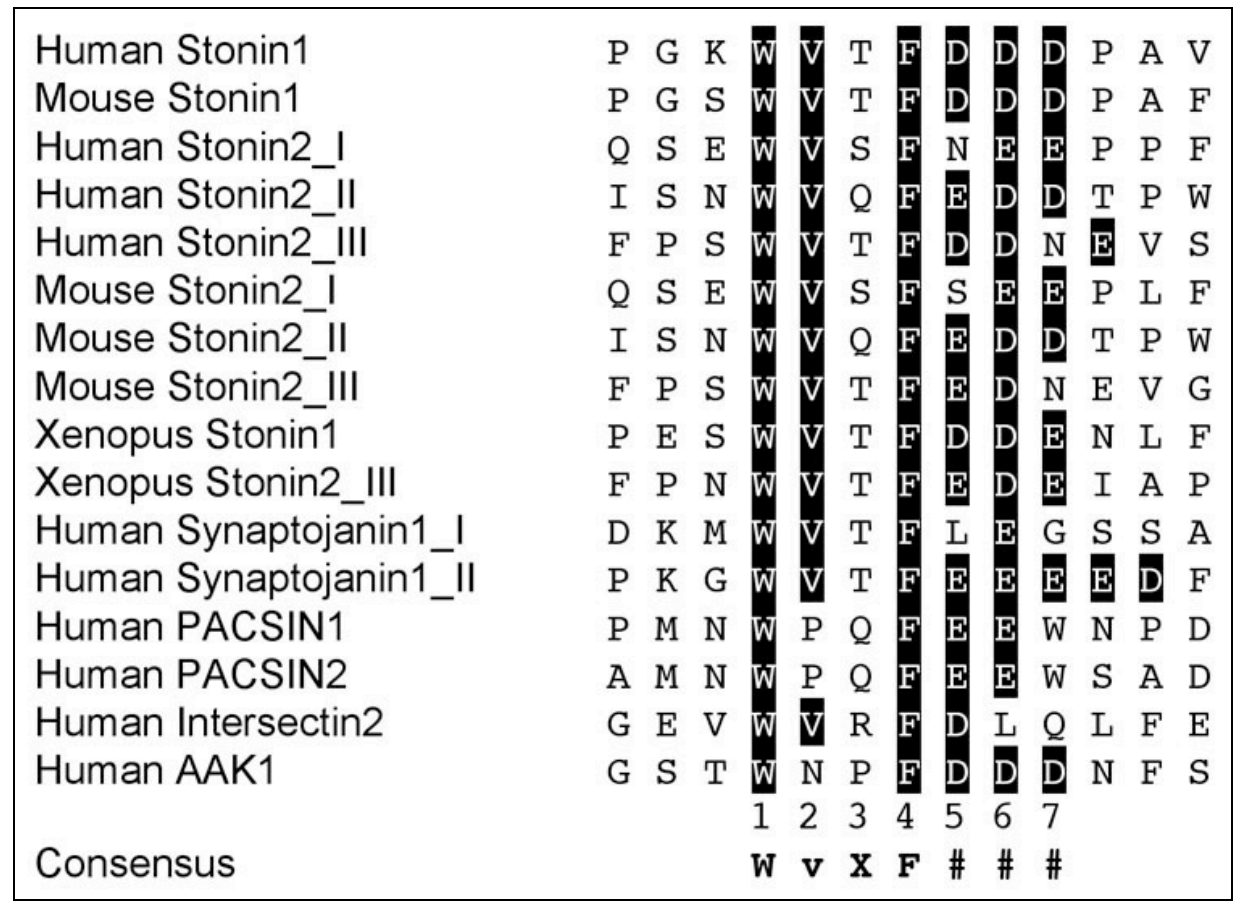

Figure 3.9 Novel AP2 interaction motif initially discovered in stonins. The novel AP2 interaction motif consists of three sequence components that are essential for binding to AP2; (i) a tryptophan at the first position (ii) a phenylalanine at the $4^{\text {th }}$ position (iii) one or more negatively charged residues at $5^{\text {th }}, 6^{\text {th }}$ and $7^{\text {th }}$ positions. Although the mutation of valine at the second position to alanine significantly inhibits AP2 binding, it is not essential (Walther et al., 2004).

In order to test for the requirement of the WVXF type motif in binding of stonin 1 to AP2 complex, we generated stonin 1 constructs with truncations lacking the single WVXF motif (residues 16-200) or containing the amino-terminal 33 residues including the WVXF sequence. A GST-Stonin $1_{(1-33)}$ fusion protein but not GST-Stonin $1_{(16-200)}$ was able to affinity purify AP2 from RBE (Figure 3.10). No interaction with AP1 could be detected by Western blot analysis. Using GST-Stonin $1_{(1-33)}$, we could purify chemical amounts of AP2 from RBE, that can be seen by Coomassie blue staining of SDS gels (Figure 3.10B).

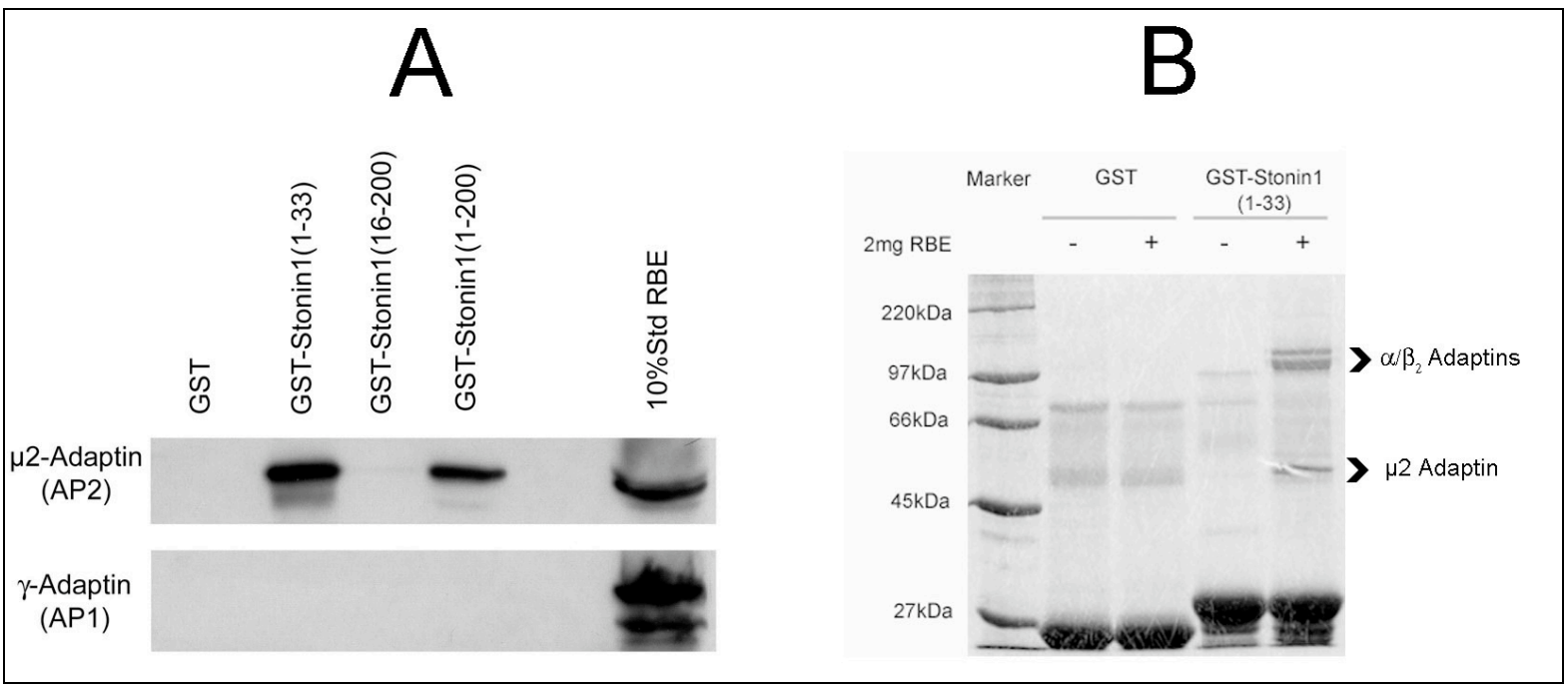


Figure 3.10 Delineation of WvXF type motifs in stonin1 sequence. (A) Mouse stonin 1 contains the WVTFDDD peptide sequence between residues 9-15. GST fusion proteins that include this region can affinity purify AP2 from the RBE. GST-Stonin $1_{(16-200)}$ has lost the binding capability to AP2. (B) Quantitative levels of AP2 can be affinity purified from the RBE using GST-Stonin $1_{(1-33)}$ construct. AP2 subunits $\alpha, \beta$ and $\mu 2$ are indicated with arrows on the figure.

Concurrent with our findings, other groups have identified the same AP2 interaction motif in NECAPs and the long-splice isoform of synaptojanin1 (Ritter et al., 2003; Jha et al., 2003). Interestingly, NECAPs also harbor a WGDF motif just 20 amino acids upstream of the AP2 binding WVQF motif, which has been shown to bind to the TGN localized clathrin adaptor AP1 (Ritter et al., 2004). The position of the negatively charged residues determines the binding specificity of these two motifs to AP1 or AP2 such that AP1 binding requires presence of negative charges upstream of the WXXF motif and AP2 binding in the downstream (Ritter et al., 2004).

\subsection{Overexpression of stonin 2 but not stonin 1 blocks CME in COS7 cells}

Overexpression of endocytic accessory proteins in eukaryotic cell lines has an inhibitory effect on clathrin-mediated endocytosis by sequesteration of other components of the endocytic machinery. For example, high level expression of a GFP-epsin2 construct in $\mathrm{CHO}$ cells causes the redistribution of its interaction partners AP2, clathrin and Eps15. This in turns leads to an inhibition of clathrinmediated endocytosis assayed by internalization of fluorescently labeled transferrin (Rosenthal et al., 1999).

The presence of three high affinity AP2 interaction motifs within the stonin 2 aminoterminal domain would assure the strong interaction of the two proteins in vivo. In order to study the functional consequences of AP2 and stonin interactions on clathrin-dependent endocytosis, we overexpressed EGFP-stonin 1 and EGFP-stonin 2 constructs in COS-7 fibroblasts. These cells do not express endogenous stonin 2 (Figure 3.3A).

As expected, overexpression of high levels of stonin 2 in COS-7 cells caused a depletion of AP2 from coated pits of the plasma membrane. In contrast, overexpression of EGFP alone (data not shown) or the EGFP-stonin 1 fusion protein had no effect on the distribution of AP2 (Figure 3.11). Thus, the presence of a single WVXF type peptide motif is insufficient for sequestration of AP2. 

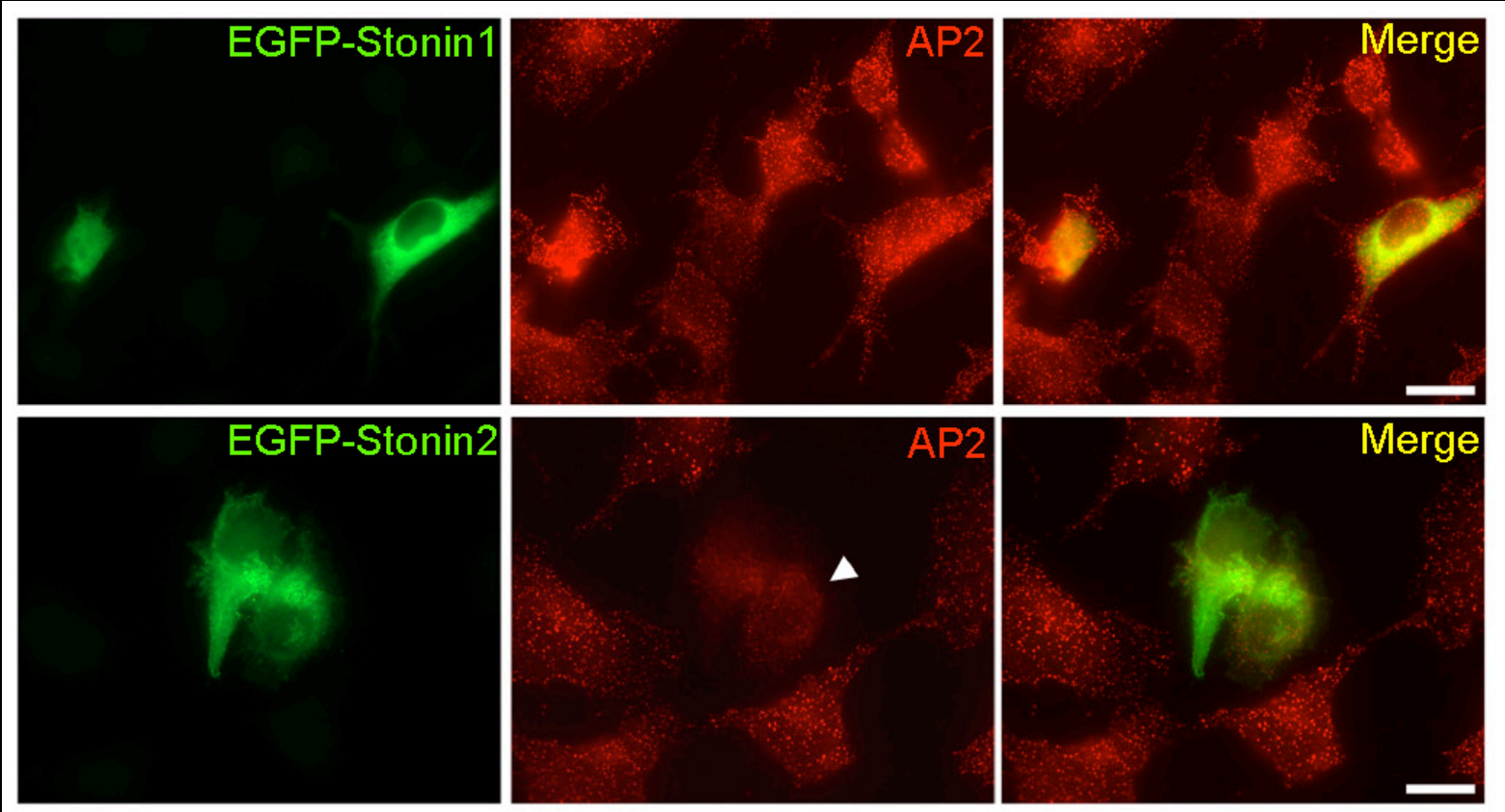

Figure 3.11 Overexpression of EGFP-stonin 2 but not EGFP-stonin 1 causes depletion of AP2 coated pits from the plasmalemma. COS-7 cells were transfected with constructs encoding EGFP, EGFP-stonin 1 and EGFP-stonin 2. $24 \mathrm{~h}$ post transfection cells were fixed and immunostained with AP2 antibodies. Overexpression of EGFP (data not shown) or EGFP-stonin 1 has no effect on plasma membrane AP2 coated pits. However, EGFP-stonin 2 causes a disruption of the punctate AP2 staining (arrow head). Scale bar $20 \mu \mathrm{m}$

Consistent with these data, clathrin-mediated endocytosis of transferrin receptor is also inhibited in cells overexpressing EGFP-stonin 2 but not EGFP or EGFP-stonin 1 (Figure 3.12).
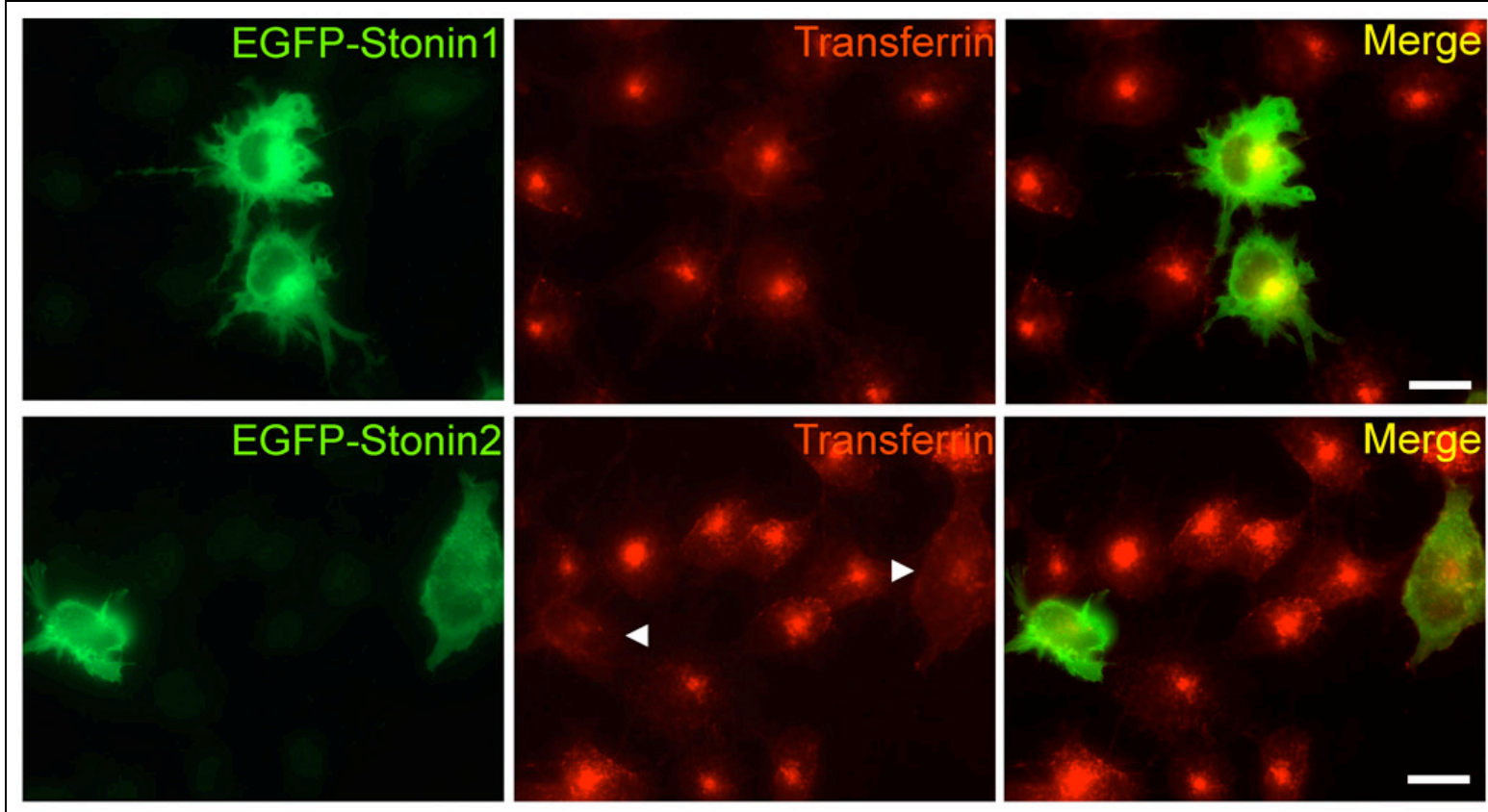
Figure 3.12 Overexpression of EGFP-stonin 2 but not EGFP-stonin 1 inhibits endocytic uptake of Texas-Red labeled transferrin. Cos-7 cells were transfected with EGFP constructs as before. Cells were starved for $3 \mathrm{~h}$ in serum-free medium (OPTIMEM) prior to extracellular application of 5 $\mu \mathrm{g} / \mathrm{ml}$ Texas-red labeled transferrin. They were allowed to internalize ligands for $15 \mathrm{~min}$ at $37^{\circ} \mathrm{C}$. Unbound transferrin was washed and cells were fixed for fluorescence microscopy analysis. EGFPStonin 1 has no negative effect on endocytosis of transferrin. However cells overexpressing EGFPStonin 2 (arrow heads) display a decreased uptake of transferrin. Scale bar, $20 \mu \mathrm{m}$.

Sequestration of AP2 and inhibition of endocytosis could be indirect effects caused by binding of stonin 2 to eps 15 through amino-terminal NPF motifs. However, mutagenesis of NPF motifs to NAV, although prevents binding of stonin 2 to eps15, does not relieve the endocytosis block or depletion of AP2 from the plasmalemmal coated pits (Walther et al., 2004). Accordingly, overexpression of a stonin 2NT truncation mutant (lacking the $\mathrm{C}$-terminal $\mu \mathrm{HD}$ ) retains its dominant negative effects with regard to endocytosis and AP2 distribution. In contrast, overexpression of a stonin 2- $\mu \mathrm{HD}$ construct lacking the entire amino-terminal domain or a full length stonin 2 construct which bears tryptophan to alanine mutations within its three WVXF motifs (Stonin $2^{\partial W W W}$ ) has no noticeable effect on AP2 distribution or transferrin endocytosis (Walther et al., 2004). These data indicate that the phenotype caused by the presence of high levels of stonin 2 in COS-7 cells is a result of its direct interaction with the AP2 complex via WVXF type motifs.

\subsection{Analysis of the stonin-synaptotagmin interactions}

The interaction of stoned/stonin proteins with synaptotagmin 1 was initially described thru the investigations of Drosophila stoned mutants. Drosophila stoned mutants demonstrate a specific synaptic mislocalization and degradation of synaptotagmin 1. The embryonic lethal phenotype seen in some stoned mutant flies can be rescued by over-expression of synaptotagmin 1 , which enhances dye uptake of synaptic vesicles in wild-type background. Moreover, in vitro interaction assays using Drosophila brain extracts indicate a physical interaction between $\mu \mathrm{HD}$ of Drosophila stoned $\mathrm{B}$ and synptotagmin1 C2B domain. (Fergestad et al., 1999 and 2001; Stimson et al., 2001; Phillips et al., 2000).

In vitro and in vivo experiments involving mammalian stonins show that stonin 2 , but not stonin 1, interacts with the C2B domains of synaptotagmin 1 and 2 via its carboxy-terminal HHD (Martina et al., 2001; Walther et al., 2001).

One approach to test whether proteins exist in a complex in vivo, is to immunoprecipitate one of the interaction partners and assay for the presence of the 
other partner(s) by immunoblotting. Stonin 2 antibodies coupled to protein $G$ sepharose beads were able to coimmunoprecipitate both AP2 and synaptotagmin 1 together with stonin 2 from RBE. By contrast, clathrin was absent from the immunoprecipitates (Figure 3.13A).

Using brain extracts obtained from stonin 2 deficient mice as a negative control, we were able to confirm these findings (Figure 3.13B).

In addition to coimmunoprecipitation experiments, we performed affinity chromatography from RBE using GST or GST- $\alpha$-adaptin appendage domain fusion protein immobilized on beads. In our previous studies, we have demonstrated that $\alpha-$ adaptin appendage domain can affinity purify stonin 2 from RBE by its interactions with the WVXF type motifs (Walther et al., 2004). In agreement with the coimmunoprecipitation data we found that both synaptotagmin 1 and AP2 are isolated together with stonin 2 on the affinity matrix (Figure 3.13C).

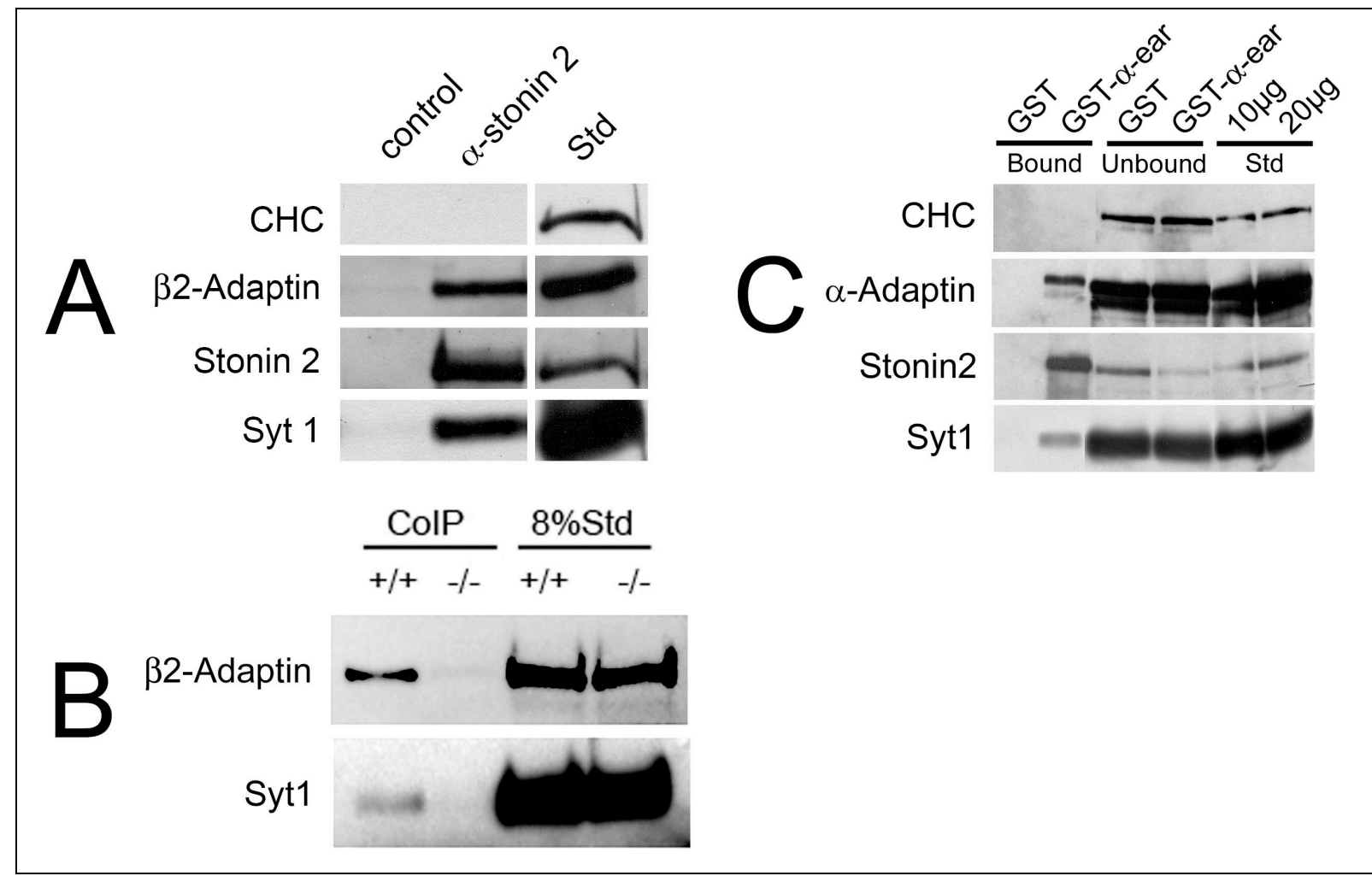

Figure 3.13 Binding of stonin 2 to synaptotagmin 1 and AP2. (A) Rat brain triton extracts were incubated with stonin 2 antibody coupled or control beads to immunoprecipitate stonin 2. Bound proteins were eluted by boiling in sample buffer and analyzed by western blotting. Standard $5 \%$. (B) ColP was performed from brain extracts of stonin 2 WT $(+/+)$ or KO (-/-) mice. (C) GST- $\alpha$-ear (appendage) domain of $\alpha$-adaptin bound beads were used in affinity pulldowns of stonin 2 from $2 \mathrm{mg}$ RBE. After incubation, beads were washed 3 times and bound proteins were eluted by boiling in

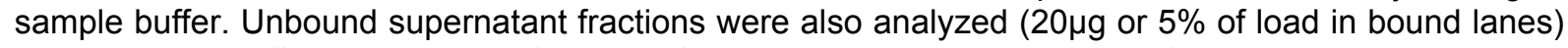
to demonstrate efficient recovery of stonin 2 from the brain extracts using the fusion proteins. 
In order to analyze the functional partnership between stonin 2 and stonin 1 in living cells, we transfected rat hippocampal neurons with constructs encoding EGFP-Stonin 1 and EGFP-Stonin 2. Stonin 1 mainly localized to the cell body and displayed a soluble distribution pattern in neurons and did not colocalize with synaptotagmin 1. However, transfected stonin 2 assumed a punctate distribution and completely colocalized with the endogenous synaptotagmin 1 at presynaptic vesicle clusters (Figure 3.14). Deletion of its carboxy-terminal $\mu \mathrm{HD}$ from stonin 2 abolishes the punctate distribution pattern and renders stonin 2 soluble (data not shown and Walther et al., 2004). This suggests that interactions of stonin 2- $\mu \mathrm{HD}$ with synaptic proteins are an important determinant for targeting the protein to synaptic sites.
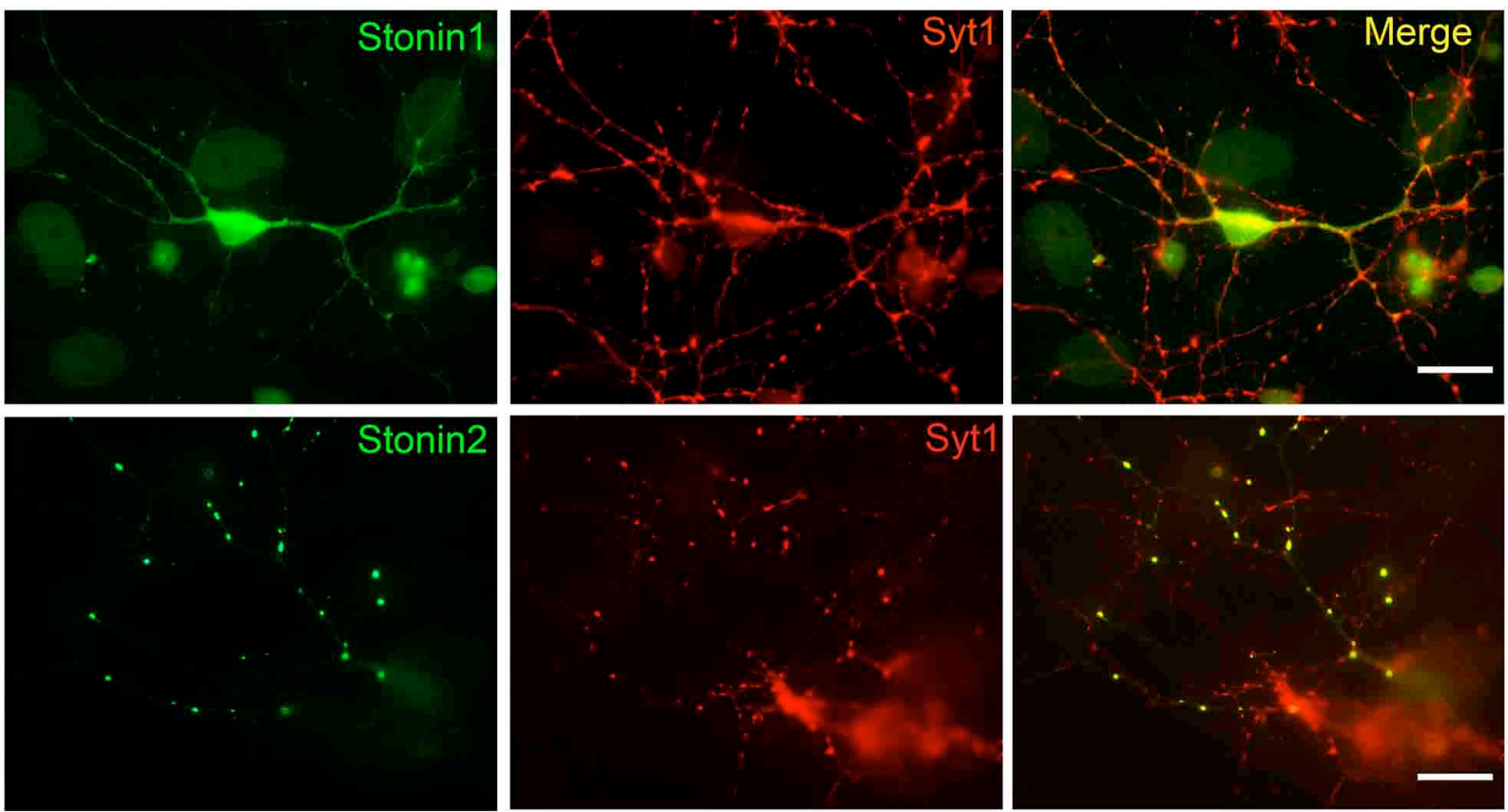

Figure 3.14 Distribution of stonin 1 and stonin 2 in neurons. Rat brain hippocampal neurons were transfected with EGFP-stonin 1 or EGFP-stonin 2 constructs by calcium phosphate-mediated DNA precipitation method. Cells were fixed and immunostained for synaptotagmin 1 one week after transfection. EGFP-stonin 1 shows a typical cytosolic distribution. EGFP-stonin 2 displays a punctate distribution and colocalizes with synaptotagmin 1. (Note that the nuclei of underlying astrocytes are also visible due to auto-fluorescence and higher exposure times required to detect the less efficiently expressed EGFP-stonin 1). Scale bar, $20 \mu \mathrm{m}$.

The differential localization of stonin 2 and a truncation mutant lacking the $\mu \mathrm{HD}$ (Stonin 2-NT) in transfected neurons implies that synaptotagmin 1 may be directly influencing the targeting of stonin 2 to membranes. In order to test this likelihood, we overexpressed full length stonin 2 or a truncation mutant lacking the $\mu \mathrm{HD}$ (Stonin 2NT), either alone or together with lumenally FLAG epitope-tagged synaptotagmin 1 , in N1E-115 neuroblastoma cells and examined the distribution of both proteins by 
confocal fluorescence microscopy. Since undifferentiated N1E-115 neuroblastoma cells do not contain any compartment(s) analogous to synaptic vesicles in neurons, overexpressed synaptotagmin 1 gets stranded on the plasma membrane.

When transfected alone, stonin 2 and stonin $2^{\mathrm{NT}}$ were both predominantly cytosolic and homogenously distributed throughout the cell. However, upon cotransfection of FLAG-tagged synaptotagmin 1, full length stonin 2 efficiently translocated to the plasma membrane where it colocalized with synaptotagmin 1. It was also possible to observe minor amounts of both stonin 2 and synaptotagmin 1 colocalizing within internal vesicular organelles. In contrast, the presence or absence of synaptotagmin1 did not have any effect on the cytosolic distribution pattern of the stonin $2^{\mathrm{NT}}$ truncation mutant, suggesting that the $\mu \mathrm{HD}$-synaptotagmin1 interaction causes the redistribution of stonin 2 in these cells (Figure 3.15).

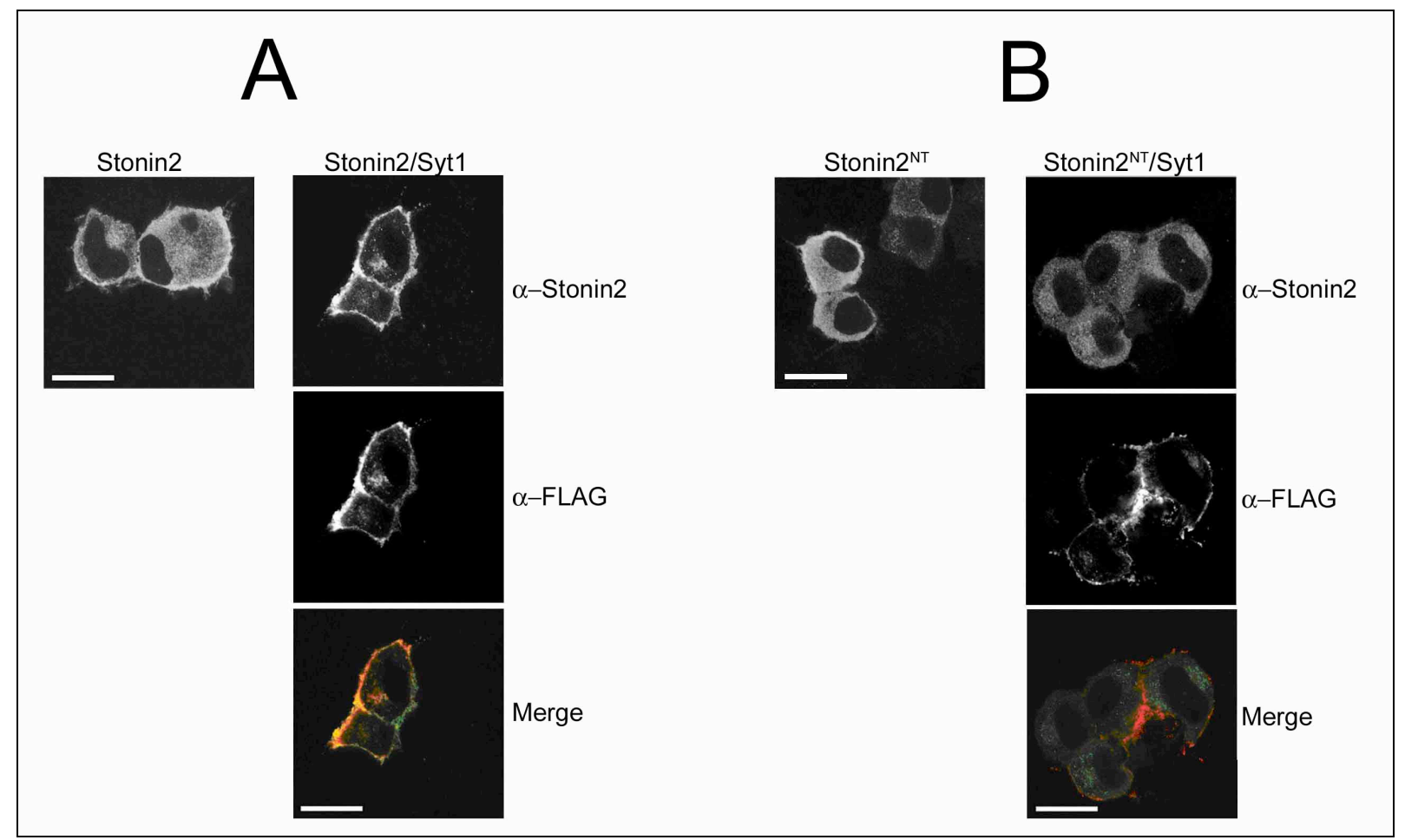

Figure 3.15 Translocation of stonin 2 to the plasmalemma in N1E cells. Undifferentiated N1E-115 cells were transfected with plasmids encoding full length stonin 2 or stonin $2^{\mathrm{NT}}$ truncation lacking the $\mu \mathrm{HD}$, either alone or together with a plasmid encoding lumenally FLAG-tagged synaptotagmin $1.24 \mathrm{~h}$ post-transfection cells were fixed and immunostained using polyclonal stonin 2 and monoclonal FLAG primary antibodies. Cells were analyzed by confocal immunofluorescence microscopy for the distribution of stonin 2, stonin $2^{\mathrm{NT}}$ (Alexa ${ }^{488}$, green) and FLAG-synaptotagmin1 (Alexa ${ }^{594}$, red). (A) Full length stonin 2 redistributes to the plasmalemma upon coexpression with synaptotagmin 1 . (B) No change in the cytosolic distribution of stonin $2^{\mathrm{NT}}$ is observable in the presence of synaptotagmin 1. Scale bar, $16 \mu \mathrm{m}$. 
Recruitment of stonin 2 to the plasmalemma upon coexpression with synaptotagmin 1 was not unique to N1E cells. We repeated the same experiment also in PC12, COS-7 and HEK293 cells and obtained very similar results (data not shown).

In order to test the putative physical and functional interactions between stonins and different synaptotagmin family members, we made use of the above described in vivo interaction assay in N1E-115 neuroblastoma cells. Both EGFP tagged stonin 1 and stonin 2 display a predominantly cytosolic distribution when overexpressed in these cells. Cotransfection of synaptotagmin 1 targeted to the plasma membrane, was sufficient to recruit EGFP-stonin 2 but not EGFP-stonin 1 to the plasmalemma. In a similar manner, overexpression of synaptotagmins 2 and 9 could also recruit stonin 2 to the plasmalemma. In the case of synaptotagmin 9 , it was possible to colocalize both proteins also on internal vesicular organelles, presumably endosomes. A weaker membrane recruitment phenotype was observed upon cotransfection of EGFP-stonin 2 with synaptotagmin 8. Synaptotagmins 3, 4, 5, 6, 7, 10, and 11 were unable to recruit stonin 2 to the plasmalemma or other internal membranes they localized to (Figure 3.16). On the contrary, none of the tested synaptotagmins was able to change the soluble, cytosolic distribution of EGFP-stonin 1 (Figure 3.17). These observations suggest that stonin 2 , but not stonin 1 , can physically interact with synaptotagmins 1, 2, 8, and 9 in living cells.

Consistent with this it has been shown that synaptotagmins 1,2 and 9 are expressed predominantly in brain and secretory, neuroendocrine cells following a parallel expression pattern to stonin 2. 15 different synaptotagmin isoforms have been identified in mammals (Südhof, 2002; Fukuda, 2003). Out of these, synaptotagmins 1, 2 and 9 show very high sequence similarity and define a separate branch of the synaptotagmin phylogenetic tree (Figure 3.18). Their genomic organizations (intronexon boundaries) are almost identical (Südhof, 2002), and they exhibit similar biochemical characteristics (i.e. $\mathrm{Ca}^{2+}$ dependent phospholipid binding activity). In invertebrates, there is only one synaptotagmin corresponding to these three synaptotagmins in mammals. Thus, the interaction between stonedB/stonin and synaptotagmin 1 in lower animals is evolutionarily conserved in higher animals although these contain 3 isoproteins of the same synaptotagmin orthologue.

Little data is available about synaptotagmin 8. Its genomic structure (exon/intron organization) resembles to synaptotagmin 1,2 and 9 but it lacks the conserved residues that make up the $\mathrm{Ca}^{2+}$ binding site (Südhof, 2002). 


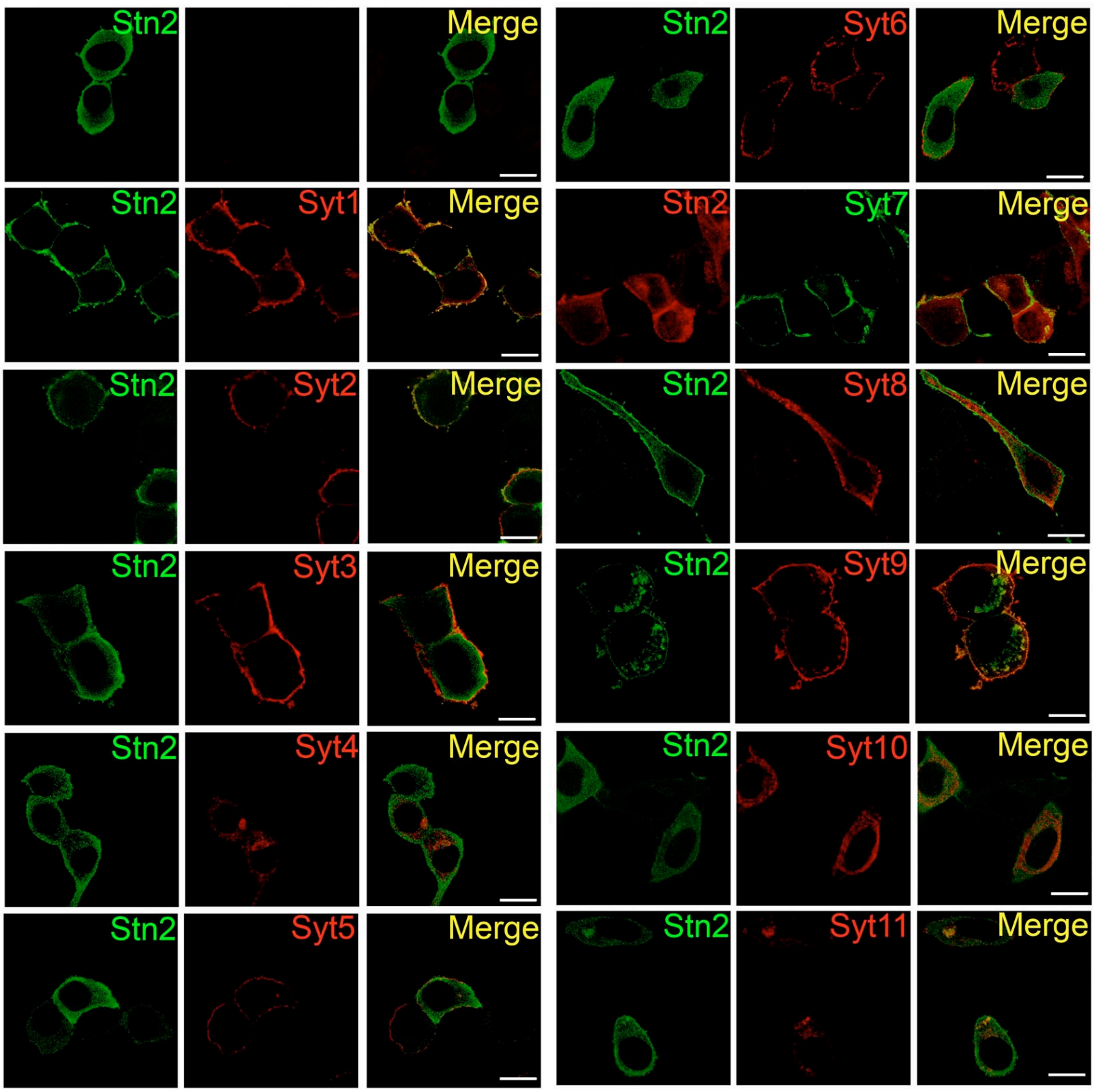

Figure 3.16 In vivo interaction assays for stonin 2 and synaptotagmins 1-11. N1E-115 cells were cotransfected with EGFP-stonin 2 (HA-stonin 2 for synaptotagmin7) and synaptotagmins 1-11 (synaptotagmins 1, 2, 4, 5, 6, 8, 10, 11 were lumenally FLAG tagged, synaptotagmins 3 and 9 were T7 tagged and synaptotagmin 7 had a GFP tag at the C-terminal domain). $24 \mathrm{~h}$ after transfection cells were fixed and analyzed by confocal fluorescence microscopy as described before. Cytosolic stonin 2 translocates to the membranes upon coexpression of synaptotagmins $1,2,8$ and 9 . Scale bar, $16 \mu \mathrm{m}$ 

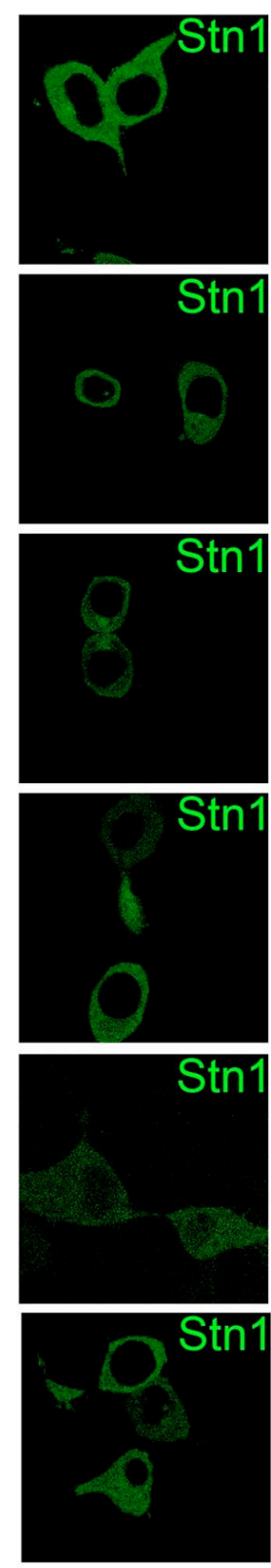
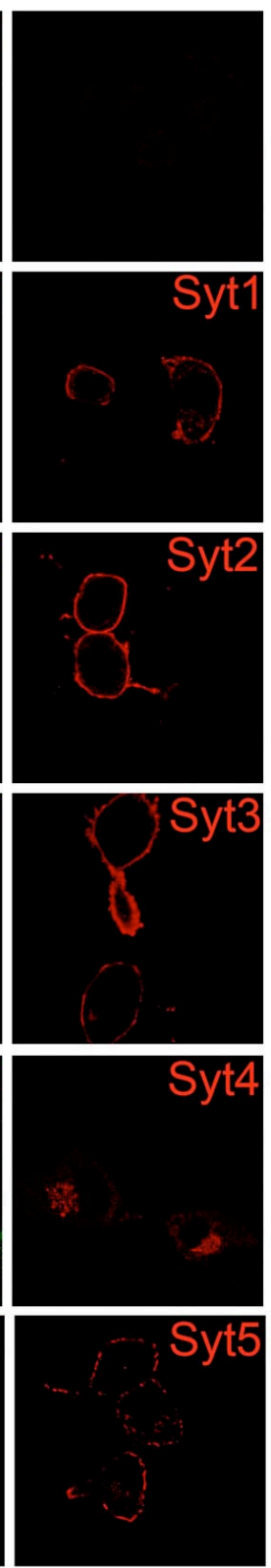
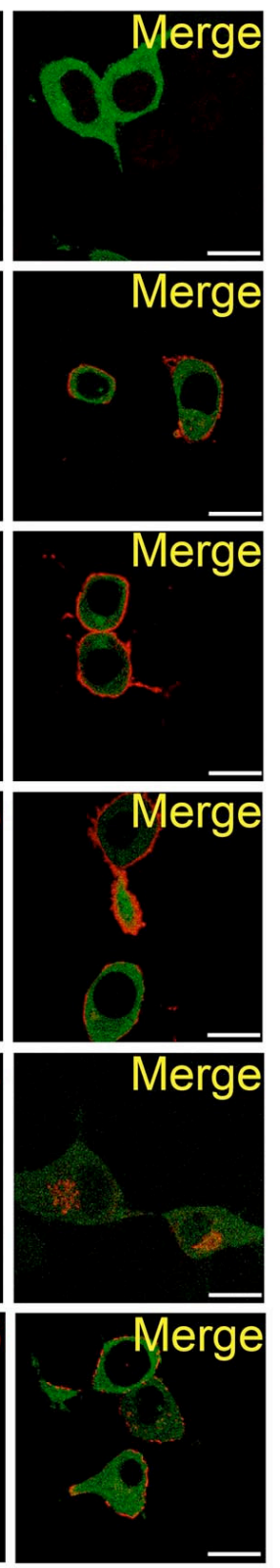
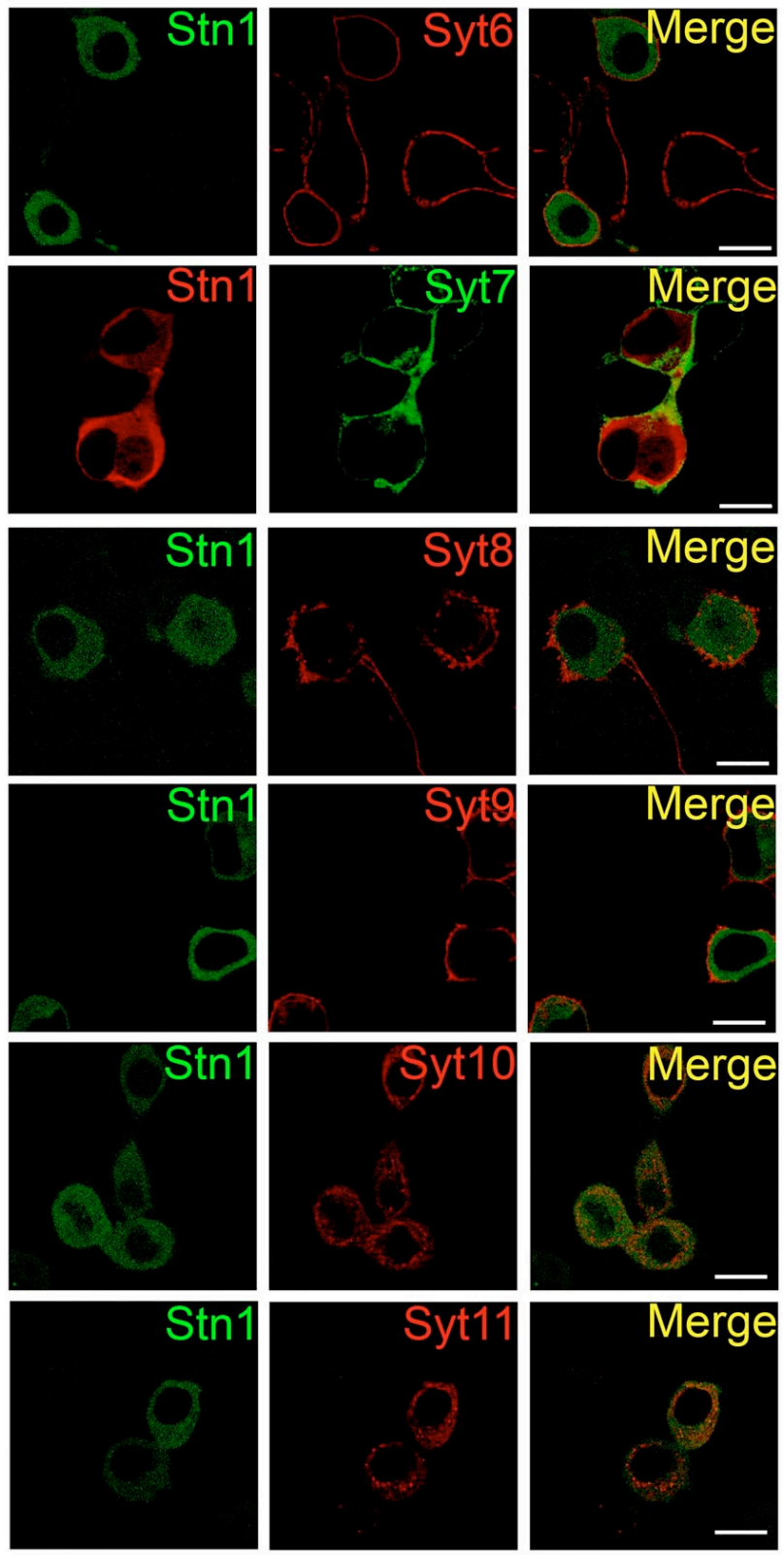

Figure 3.17 In vivo interaction assays for stonin 1 and synaptotagmins 1-11. N1E-115 cells were cotransfected with EGFP-stonin 1 (HA-stonin 1 for synaptotagmin 7) and synaptotagmins 1-11 (synaptotagmins 1, 2, 4, 5, 6, 8, 10, 11 were lumenally FLAG tagged, synaptotagmins 3 and 9 were T7 tagged and synaptotagmin 7 had a GFP tag at the C-terminal domain). $24 \mathrm{~h}$ after transfection cells were fixed and analyzed by confocal fluorescence microscopy as described before. The distribution of stonin 1 does not change and it remains cytosolic when coexpressed with the indicated synaptotagmins. Scale bar, $16 \mu \mathrm{m}$

\subsection{Stonin 2 facilitates AP2 dependent endocytosis of synaptotagmin 1}

In the previous section, we have shown that stonin 2 and synaptotagmin 1 can interact in vivo. This interaction is dependent on the presence of the $\mu \mathrm{HD}$ in stonin 2. 


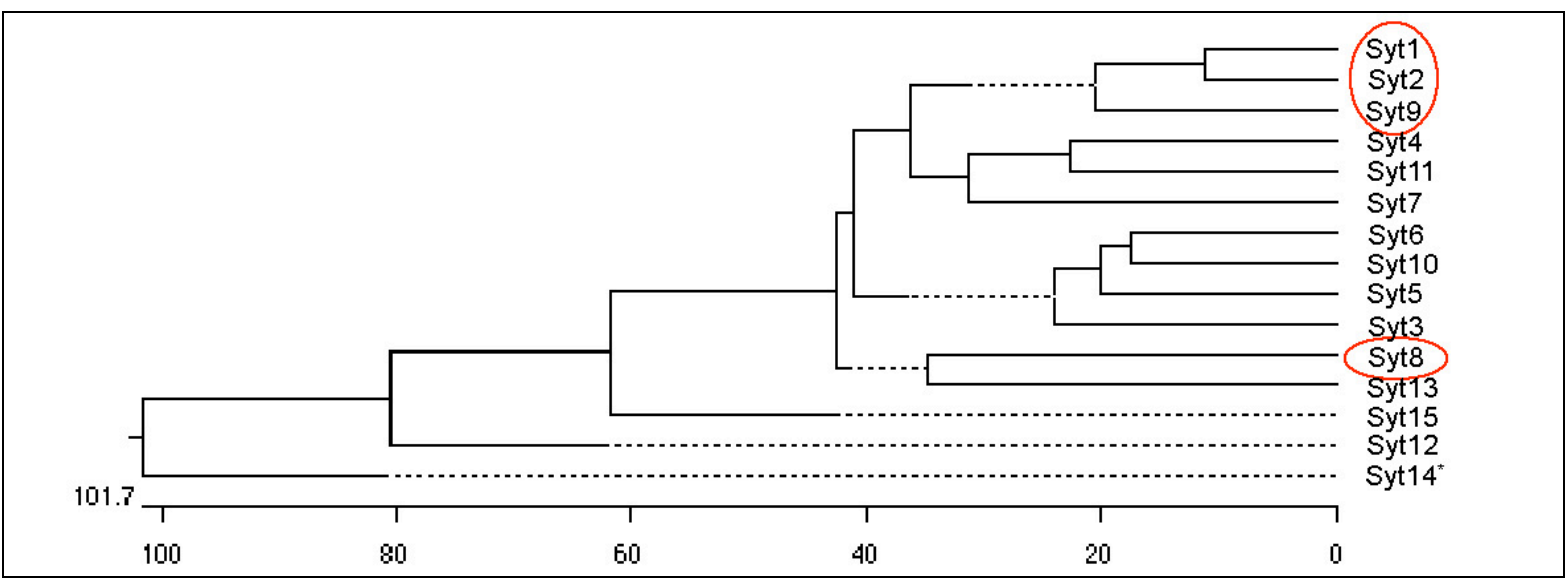

Figure 3.18 Phylogenetic tree of synaptotagmins. Amino acid sequences of rat synaptotagmins (mouse sequence for synaptotagmin 14) were aligned according to the clustal algorithm of Megalign program. Synaptotagmins interacting with stonin 2 in vivo are circled.

Upon careful analysis of stonin 2/synaptotagmin 1 cotransfected N1E-115 cells by epifluorescence microscopy, we noticed that the distribution of stonin 2 at the plasma membrane is non-homogenous and represented puncta reminiscent of clathrin/AP2coated pits (Figure 3.19A). Double immunostaining of stonin 2/synaptotagmin 1 cotransfected cells using antibodies against stonin 2 and AP2 revealed a near perfect colocalization of the two proteins within the plasma membrane coated pits (Figure 3.19B). In contrast to COS-7 cells, overexpression of stonin 2 in N1E cells did not cause a significant depletion of AP2 coated pits from the plasmalemma, except those cells displaying very high expression levels of stonin 2 (data not shown). In contrast to stonin 2, synaptotagmin 1 showed a uniform, non-punctate distribution on the plasma membrane. In some N1E cells which expressed very low levels of stonin 2, we noticed a punctate distribution of stonin 2 event in the absence of transfected synaptotagmin, suggesting that other factors or levels of endogenous synaptotagmin can facilitate membrane recruitment. How exactly synaptotagmin 1 causes stonin 2 to translocate to AP2-coated pits remains to be analyzed. 

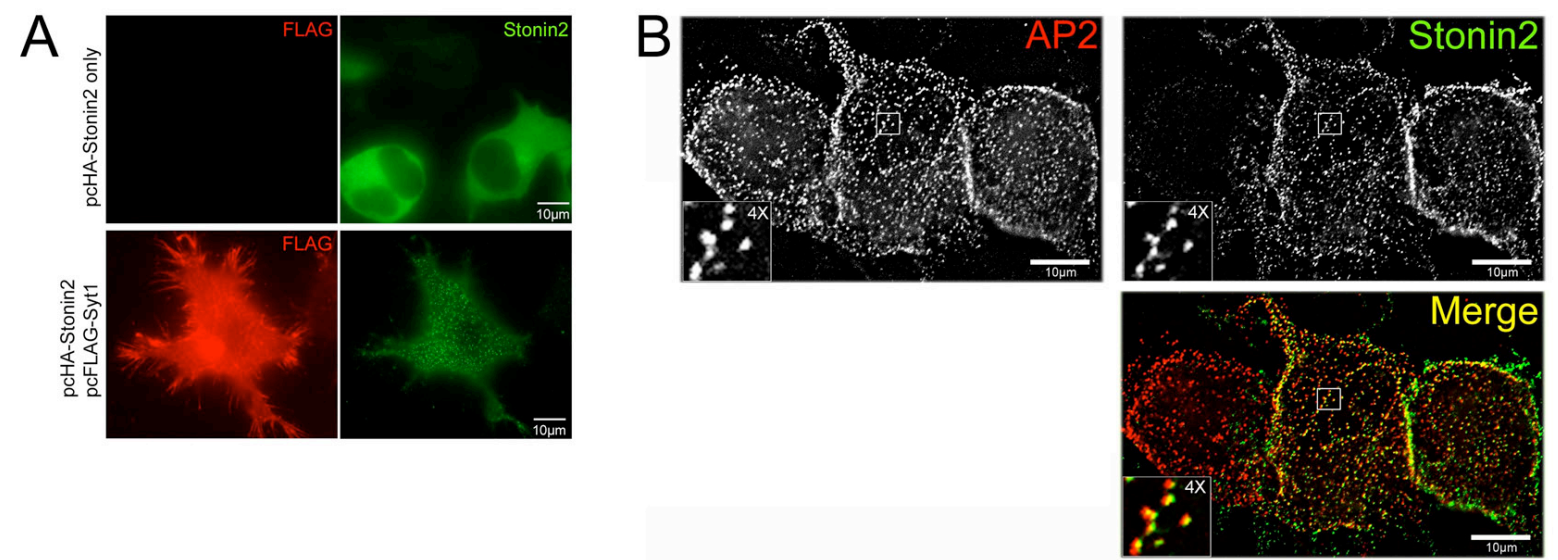

Figure 3.19 Translocation of stonin 2 to AP2 coated pits in N1E cells. (A) N1E cells were transfected with pcHA-stonin 2 either alone or in presence of pcFLAG-synaptotagmin1 construct. Cells were fixed and immunostained with antibodies against stonin 2 (green) and FLAG epitope (red). Otherwise soluble stonin 2 assumes a punctate distribution on the plasma membrane when coexpressed together with synaptotagmin1. (B) N1E cells cotransfected with stonin 2 and synaptotagmin 1 as in A were immunostained with antibodies against stonin 2 (green) and AP2 (red). Serial sections of the images were taken along the z-axis and image files were deconvolved to produce the higher quality images. Stonin 2 colocalizes with AP2 on the plasma membrane of costransfected cells. Inset shows $4 \mathrm{X}$ magnification of the selected area.

Synaptotagmin 1 has been proposed to be a high affinity receptor on synaptic vesicle membranes for AP2 (Zhang et al., 1994). The AP2-synaptotagmin 1 interaction is mediated by the C2B domain of synaptotagmin 1 and is independent of $\mathrm{Ca}^{2+}$ concentration. All tested synaptotagmins were shown to be high affinity AP2 interactors with $\mathrm{K}_{\mathrm{D}}$ values around 0.1-1 nM (Li et al., 1995). However, overexpression of synaptotagmin 1 in fibroblast cell lines results in accumulation of synaptotagmin 1 on the plasma membrane although coexpressed synaptic vesicle proteins synaptophysin and SV2 localize to distinct vesicular compartments (Feany et al., 1993a). Synaptotagmin 1 targeting to the plasma membrane and its inability to undergo clathrin/AP2-dependent endocytosis in fibroblasts suggest that other additional factors, including stonin 2 may regulate internalization in living cells.

Thus, we hypothesized that stonin 2 might facilitate endocytosis of synaptotagmin 1 in fibroblasts.

To test this hypothesis, we transfected HEK-293 fibroblasts with a construct encoding synaptotagmin 1 containing a lumenal domain FLAG epitope-tag. As reported before (Feany et al., 1993a and b), synaptotagmin 1 localized to the plasma membrane and was targeted to filopodia-like protrusions at the cell surface (data not shown). To follow its endocytic trafficking, we labeled surface-exposed synaptotagmins from the extracellular side using antibodies against FLAG epitope-tag, and incubated the cells for $2 \mathrm{~h}$ at $37^{\circ} \mathrm{C}$ for internalization. At the end of this period, all of the anti-FLAG 
antibody remained bound to the cell surface and we could not detect any intracellular compartments containing the antibody (Figure 3.20 first row). However, when the same assay was repeated with cells coexpressing stonin 2 together with synaptotagmin 1, we observed significant internalization of FLAG antibodies. At the end of the incubation period, cells had internalized most if not all of the extracellularly applied anti-FLAG antibody into a perinuclear vesicular compartment (Figure 3.20). Stonin 2 colocalized with both plasmalemmal as well as internalized synaptotagmin 1.

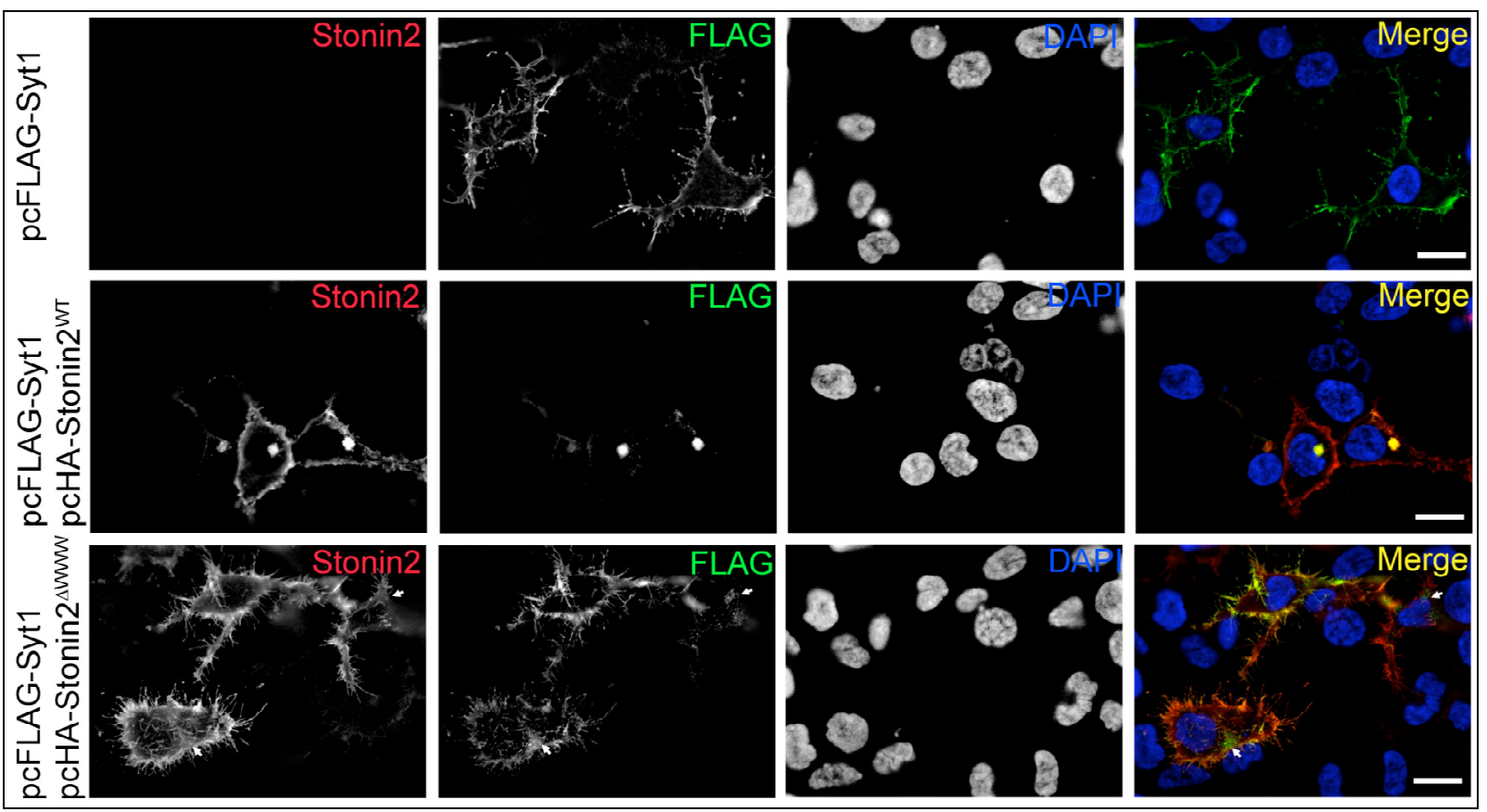

Figure 3.20 Stonin 2 facilitates endocytosis of plasmalemma stranded synaptotagmin 1 in an AP2 dependent manner. HEK293 cells expressing lumenally FLAG-tagged synaptotagmin 1 were labeled with anti-FLAG antibodies for $30 \mathrm{~min}$ on ice followed by an incubation for $2 \mathrm{~h}$ at $37^{\circ} \mathrm{C}$. Coexpression of stonin $2^{\text {WT }}$ but not a mutant in which its three WVXF motifs had been inactivated by W-to-A triple point mutations (stonin2 ${ }^{\partial W W W}$ ) facilitates endocytosis of synaptotagmin 1 and sorting to perinuclear endosomes. Stonin $2^{\partial W w W}$ however does colocalize with synaptotagmin 1 at the plasma membrane. White arrows in the bottom panel indicate cells that internalize small amounts of FLAGsynaptotagmin 1 due to stonin $2^{\partial W w W}$ coexpression. Images were obtained by nearest neighbour deconvolution of three planes separated by $0.5 \mu \mathrm{m}$ along the $\mathrm{z}$-axis. Scale bar, $16 \mu \mathrm{m}$.

The capability of stonin 2 to facilitate synaptotagmin 1 endocytosis in HEK293 cells was dependent on its interaction with AP2. A stonin 2 mutant, stonin $2^{\partial W w w}$, whose all $\alpha$-adaptin appendage domain binding motifs had been mutationally inactivated by, was unable to facilitate synaptotagmin 1 endocytosis. Nevertheless, it retained the ability to bind synaptotagmin 1 and localize to the plasma membrane (Figure 3.20, third row). In agreemant with this, we were able to colPboth proteins from extracts of transfected cells (data not shown). 


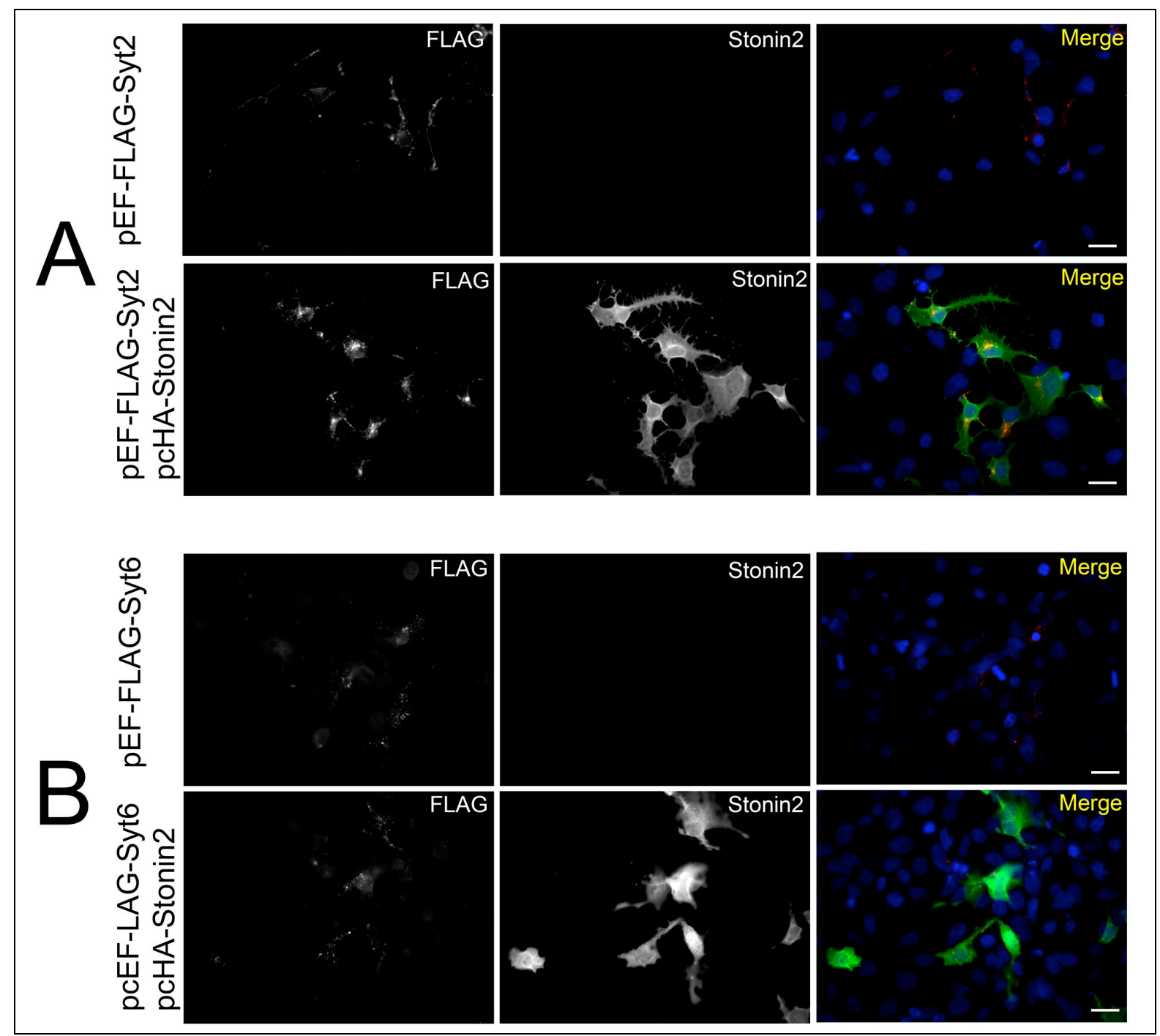

Figure 3.21 Stonin 2 facilitates endocytosis of synaptotagmin 2 but not synaptotagmin 6 . HEK293 cells were transfected with FLAG-tagged synaptotagmin 2 (A) or synaptotagmin 6 (B) in the presence or absence of stonin 2. FLAG antibody internalization assays were performed as described in legend to figure 3.28, however cell surface bound primary antibodies were blocked by goatamouse unlabeled secondary antibodies prior to further immunostaining. FLAG (red) and stonin 2 (green). Scale bar, $20 \mu \mathrm{m}$.

As in N1E cells, overexpression of synaptotagmins 2 and 6 in HEK293 cells resulted in targeting of both proteins both proteins to the plasmalemma (data not shown). We tested whether stonin 2 would facilitate internalization of these two proteins using our antibody uptake assay. Coexpression of stonin 2 in HEK cells assisted internalization of synaptotagmin 2 but not synaptotagmin 6 confirming the results of our in vivo colocalization assays in N1E cells (Figure 3.21).

In order to quantify synaptotagmin 1 endocytosis mediated by stonin 2 or mutants thereof, we made use of a HEK293-FLAG-Syt1 stable cell line (clone \#2). These cells were used at early passages (up to \#8) to assure a relatively uniform synaptotagmin 1 expression level. HEK293-FLAG-Syt1 cells were transfected with stonin 2 
constructs and anti-FLAG antibody uptake assays were performed as explained before. However, after the cells were fixed, surface bound-antibodies were blocked using a goat serum raised against mouse IgGs ( $\mathrm{H}+\mathrm{L}$ chain). Efficient blocking of the surface-bound antibodies thus allowed us to quantify the fluorescence signal derived from only internalized antibodies after permeabilization.

HEK293-FLAG-Syt1 cells internalize minimal amounts of synaptotagmin 1 in the absence of exogeneously expressed stonin 2 . Internalization is stimulated more than 10 times by expressing stonin $2^{W T}$. Expression of a stonin $2^{\partial W W W}$ AP2 binding mutant is considerably less efficient. A stonin $2^{\mathrm{NT}}-\mu 2$ chimeric construct, comprised of aminoterminal 1-562 residues of stonin 2 and carboxy-terminal domain of $\mu 2, C-\mu 2$ (residues165-434) surprisingly did not facilitate synaptotagmin 1 endocytosis (Figure 3.22). In contrast to stonin 2, overexpression of the EGFP-stonin 1 construct had no effect on endocytosis of synaptotagmin 1 (data not shown).

The results of the experiments described above suggest that stonin 2 might be facilitating synaptotagmin 1 internalization by physically linking it to AP2 and therefore to the endocytic machinery. To directly test this, we performed immunoprecipitation experiments of HEK293-FLAG-Syt1 cells transfected with stonin $2^{W T}$ or stonin the $2^{\partial W W W}$ mutant. Antibodies against AP2 were able to immunoprecipitate synaptotagmin 1 from stonin $2^{\mathrm{WT}}$ transfected cells. By contrast, no synaptotagmin 1 could be detected in AP2-precipitates derived from untransfected or stonin $2^{\partial W W W}$ mutant expressing cells (Figure 3.23). These results suggest that, stonin 2 is indeed a physical linker between synaptotagmin 1 and AP2, at least in our reconstituted cellular system.

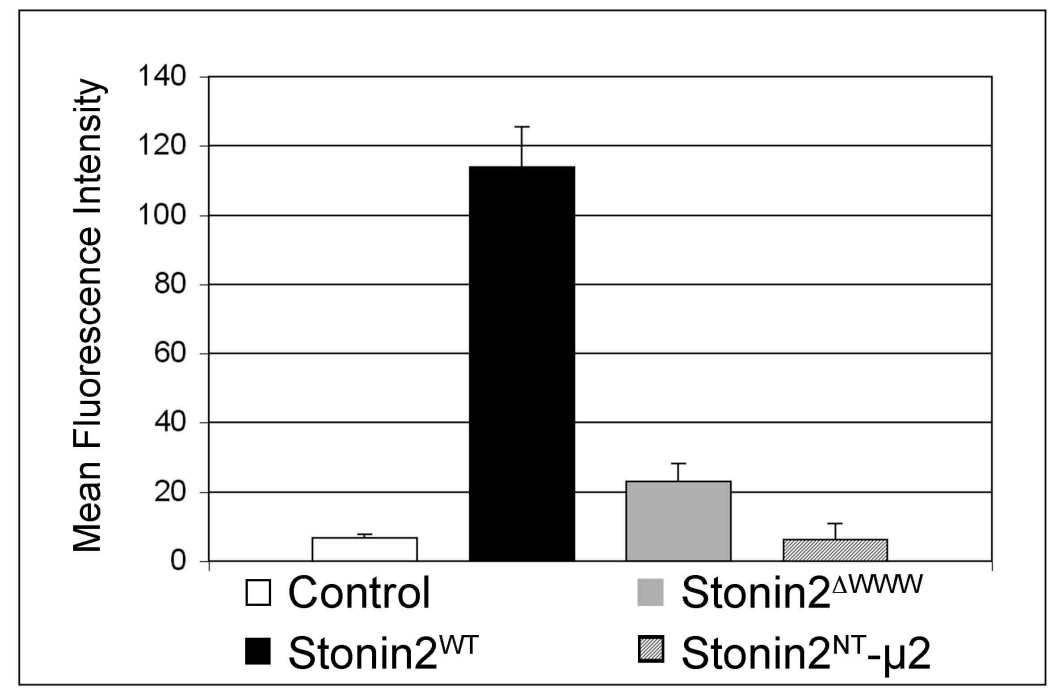


Figure 3.22 Quantification of synaptotagmin 1 internalization by various stonin 2 constructs. HEK293 -FLAG-Syt1 cells were transfected with i) mock, ii) stonin $2^{W T}$, iii) stonin $2^{\partial W w w}$, or iv) stonin $2^{\mathrm{NT}}-\mu 2$ ( a chimera in which the $\mu \mathrm{HD}$ of stonin 2 had been replaced with that of $\mu 2$-adaptin) expressing plasmids. Antibody uptake assays were performed as described before. Cell surface bound antibodies were blocked with goat-anti-mouse IgGs after fixation and cells were stained with stonin 2 antibodies followed by fluorescently labeled secondary antibodies. Internalized FLAG antibody was quantified from fluorescence microscopy images using Slidebook software. Mean fluorescence intensities of nine data sets from three independent experiments were used for calculations. Error bars represent standard deviations of these data sets. (Control: $6.69 \pm 1.05$, Stonin $2^{\mathrm{WT}}: 113.82 \pm 11.59$, Stonin $2^{\partial W W W}$ : $22.90 \pm 5.44$, Stonin $\left.2^{\mathrm{NT}}-\mu 2: 6.27 \pm 4.46\right)$

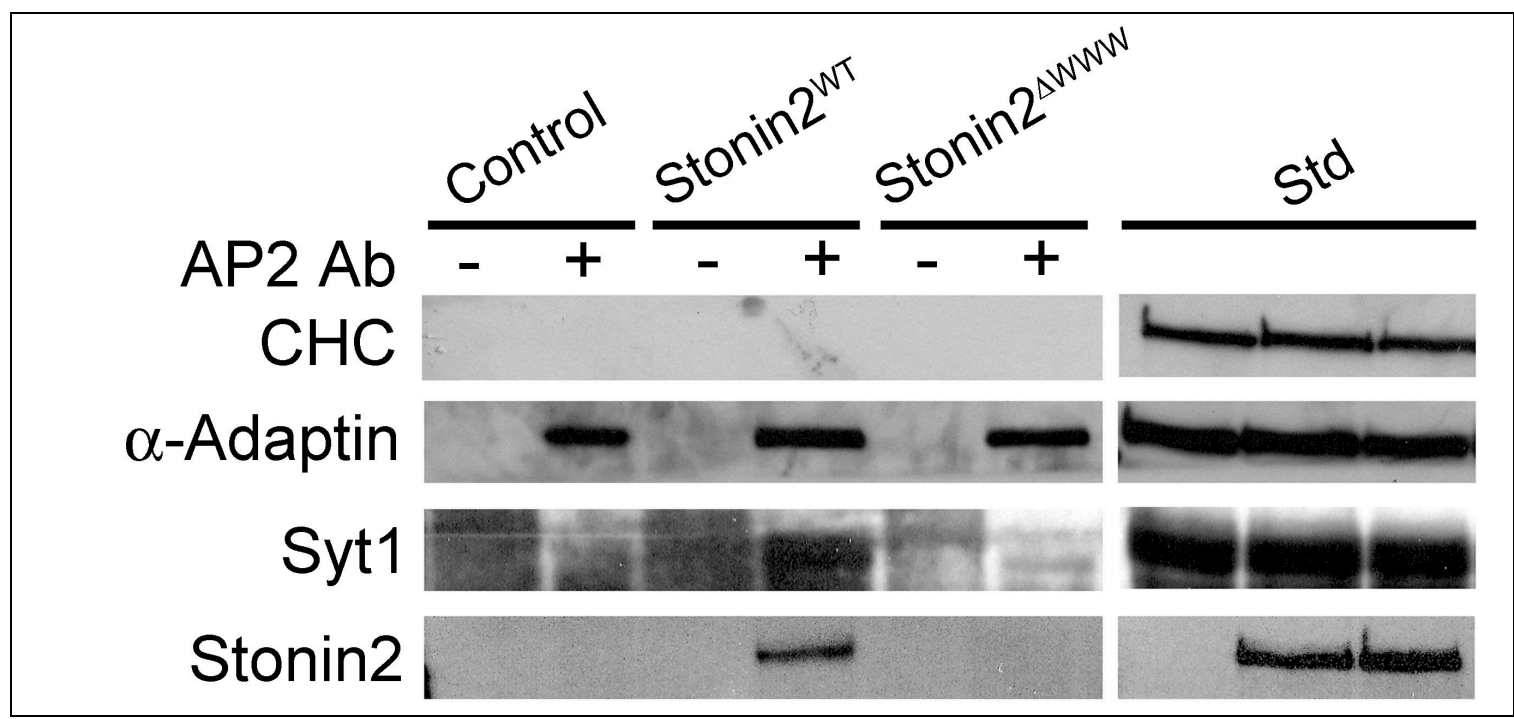

Figure 3.23 Co-Immunoprecipitation of synaptotagmin 1 only in presence of stonin 2. HEK293FLAG-Syt 1 cells were transfected with i) mock; ii) stonin $2^{\mathrm{WT}}$; iii) stonin $2^{\partial W W W}$ constructs. Cellular protein extracts were incubated with control beads or AP2 antibody-coupled beads. Synaptotagmin 1 was coprecipitated with AP2 only when the cells had been transfected with stonin ${ }^{\mathrm{WT}}$ but not with stonin $2^{\partial W W W}$. Standard, $4 \%$ of total material used for immunoprecipitation.

Stonin 2 binds to synaptotagmin 1 via its $\mu \mathrm{HD}$ and this interaction seems to be stronger than that of AP2- $\mu 2$ at least under our experimental conditions. Therefore, we decided to engineer chimeras between the AP2 complex and stonin 2. We created a chimeric construct, $\mathrm{BBD}_{\mu 2}-\mu \mathrm{HD}_{\mathrm{Stn} 2}$, that harbors the amino-terminal 164 residues of $\mu 2$ that are required for binding to AP2- $\beta_{2}$ (Beta binding domain), and carboxy-terminal $\mu \mathrm{HD}$ (563-898 residues) of stonin 2 (See Figure 3.24 A and B). A hemagglutinin (HA) epitope tag was introduced into the stonin 2- $\mu \mathrm{HD}$, between

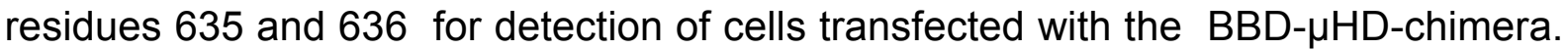
A similar site in the $\mu 2$ sequence (between residues 236 and 237) had been used for detection of transfected $\mu 2$ in previous studies (Nesterov et al., 1999; Rohde et al., 2002). Transfection of HEK293-FLAG-Syt1 cells with this chimeric contruct was sufficient to allow internalization of synaptotagmin 1, however to a slightly lesser extent compared to stonin $2^{\mathrm{WT}}$ construct transfections (Figure $3.24 \mathrm{C}$ ). Unfortunately, the transfection and expression efficiencies of our BBD- $\mu \mathrm{HD}$ construct were quite low 
compared to stonin $2^{\mathrm{WT}}$, which prevented us from directly demonstrating its incorporation into the AP2 complexes (i.e. by ColP experiments).

RNA interference is a recently developed technique for targeted degradation of specific mRNAs and knock-down of their translation products in eukaryotic cells (Elbashir et al., 2001). Expression of AP2 and clathrin subunits were successfully knocked down in previous studies (Motley et al., 2003; Hinrichsen et al., 2003). In order to further analyze the AP2 dependence of synaptotagmin 1 endocytosis facilitated by stonin 2, we decided to knock-down expression of AP2 by siRNAs against its $\mu 2$ subunit (Motley et al., 2003).

Treatment of the HEK293-FLAG-Syt1 cells with siRNAs against AP2- $\mu 2$ caused a dramatic decrease in the expression levels of $\mu 2$ and $\alpha$-adaptins. In contrast, the level of stably expressed FLAG-synaptotagmin1 or clathrin heavy chain remained unaltered (Figure 3.25A). Furthermore, in more than $80 \%$ of the cells transfected with $\mu 2$ siRNAs we were unable to detect AP2 in coated pits. These cells were also unable to internalize externally added transferrin (Figure 3.25B).

We then tested whether stonin 2 would facilitate the internalization of synaptotagmin 1 in cells where AP2- $\mu 2$ expression was knocked down by RNAi. For this purpose, we transfected HEK293-FLAG-Syt1 cells with the pcHA-stonin $2^{\mathrm{WT}}$ construct in the presence or absence of $\mu 2$ siRNAs. Stonin 2 expression in a AP2 knock-down background was inefficient to facilitate synaptotagmin 1 internalization. By contrast, mock transfected control cells displaying normal levels of AP2 could internalize synaptotagmin 1 into endosomal compartments (Figure 3.26). 


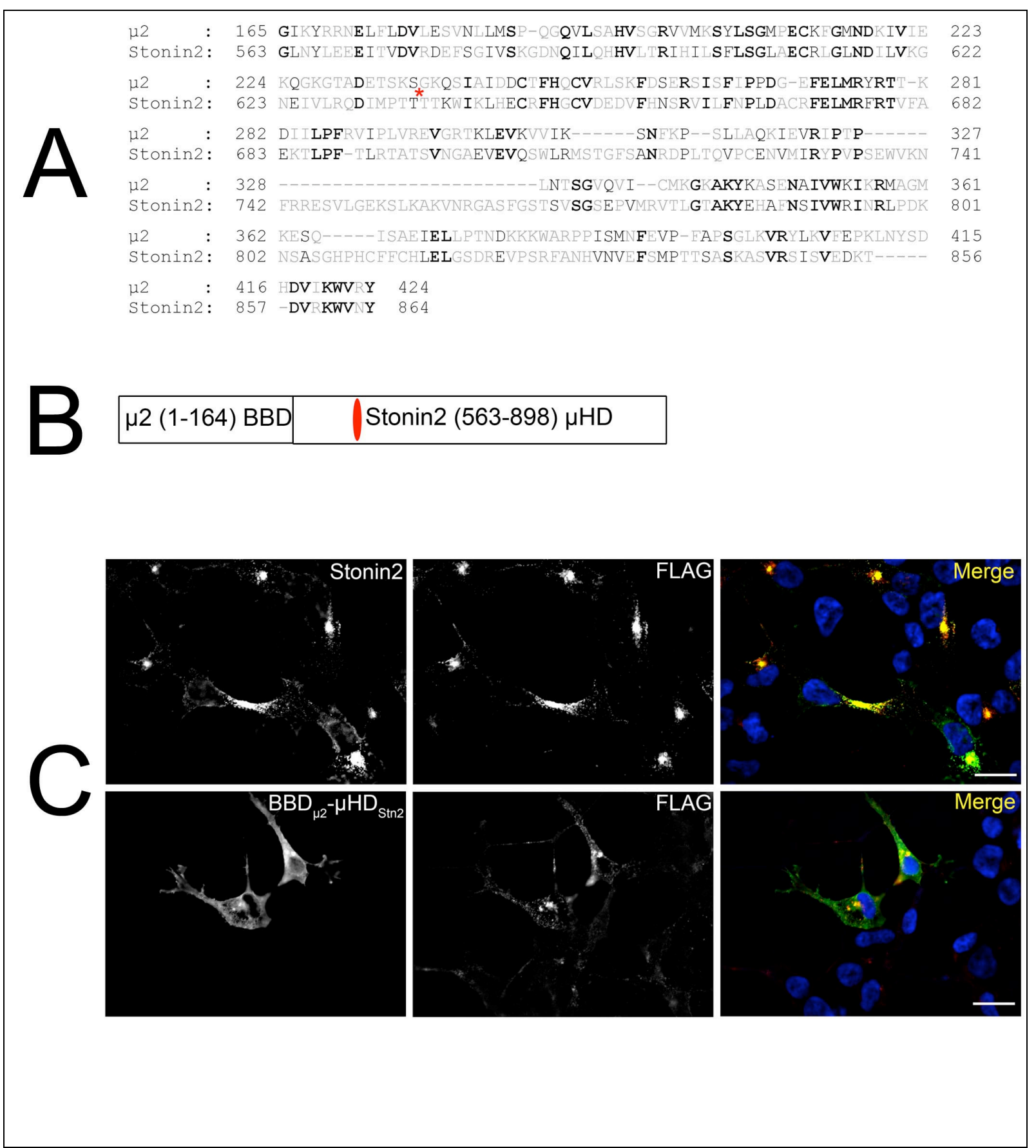

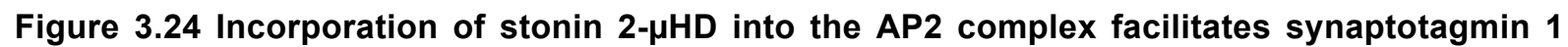
uptake. (A) Alignment of human stonin 2 and rat $\mu 2$-adaptin amino acid sequences. (B) For incorporation of stonin 2- $\mu \mathrm{HD}$ into the AP2 complex, a chimeric construct comprising the $\mu 2$-beta binding domain (BBD, residues 1-164) fused to the stonin $2 \mu$-homology domain ( $\mu \mathrm{HD}$, residues $563-$ 898) was generated. Red ellipse shows the location of the HA tag. (C) HEK293-FLAG-Syt 1 cells were transfected with stonin $2^{\mathrm{WT}}$ or BBD- $\mu \mathrm{HD}$ chimeric constructs. Antibody uptake assays and blocking of surface staining were performed as described before. Overexpression of the BBD- $\mu \mathrm{HD}$ construct stimulates synaptotagmin 1 endocytosis, albeit slightly less efficiently than stonin2 ${ }^{\text {WT }}$. Scale bar, $20 \mu \mathrm{m}$. 


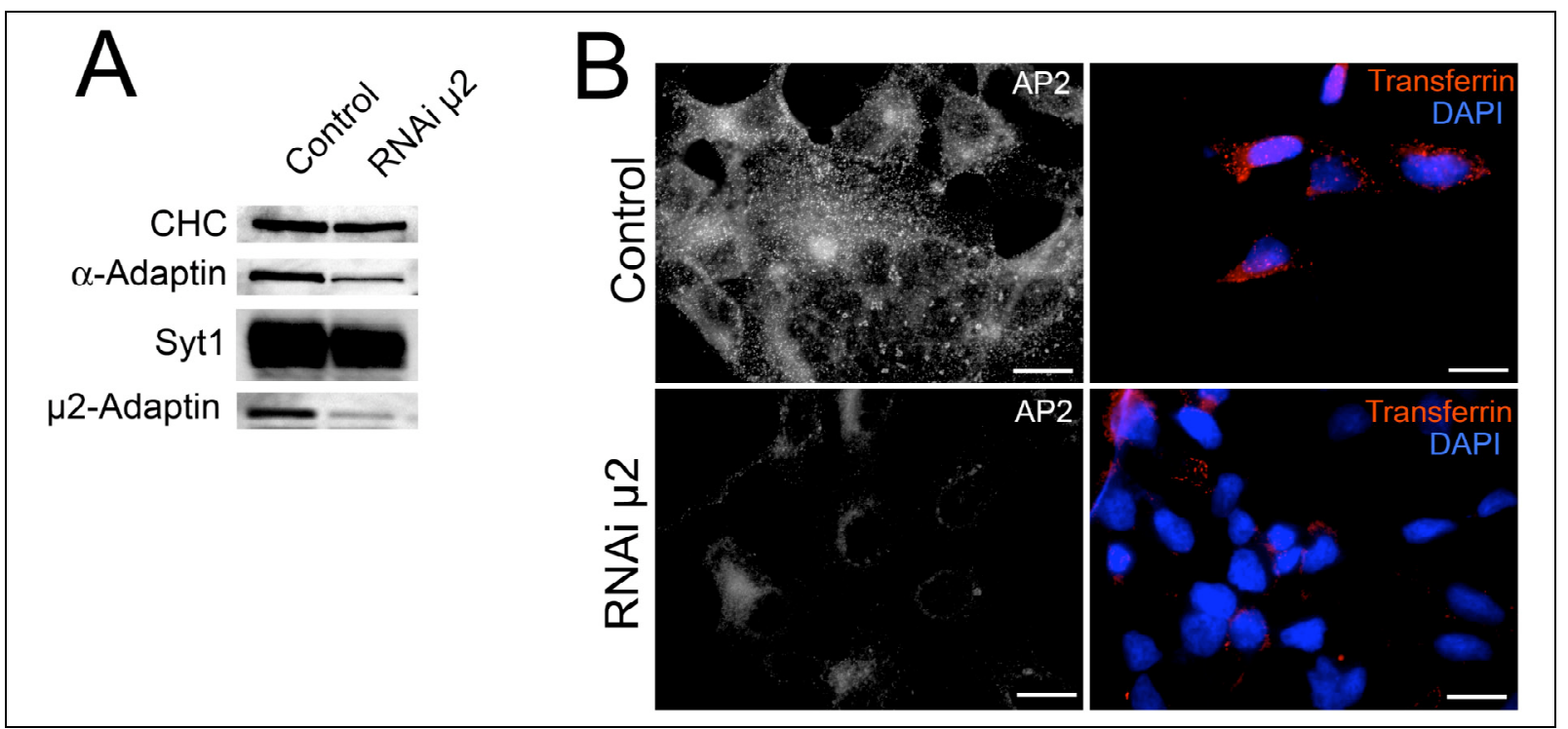

Figure 3.25 RNAi mediated knock-down AP2- $\mu 2$ in HEK cells causes depletion of AP2-coated pits and an inhibition of transferrin uptake. (A) HEK293-FLAG-Syt 1 cells were transfected twice during 96 hours with siRNAs against human $\mu 2$-adaptin. Consistent with previous results (Motley et al., 2003), $\mu 2$ knock-down causes degradation of other AP2 subunits including $\alpha$-adaptin but has no effect on clathrin or synaptotagmin 1 levels. (B) RNAi mediated knock-down causes a loss of AP2 coated pits from the plasma membrane and an inhibition of transferrin uptake. HEK293-FLAG-Syt1 cells were transfected with siRNAs against $\mu 2$ twice and cultured for 96 hours after the first transfection. For transferrin uptake assays, cells were incubated in OPTIMEM containing $20 \mu \mathrm{g} / \mathrm{ml}$ Alexa594 labeled human serum transferrin on ice for 30 minutes and warmed up to $37^{\circ} \mathrm{C}$ for 5 minutes. Note that a few cells (5-10\% of total) were able to internalize transferrin due to incomplete AP2 knock-down. Scale bar, $20 \mu \mathrm{m}$.

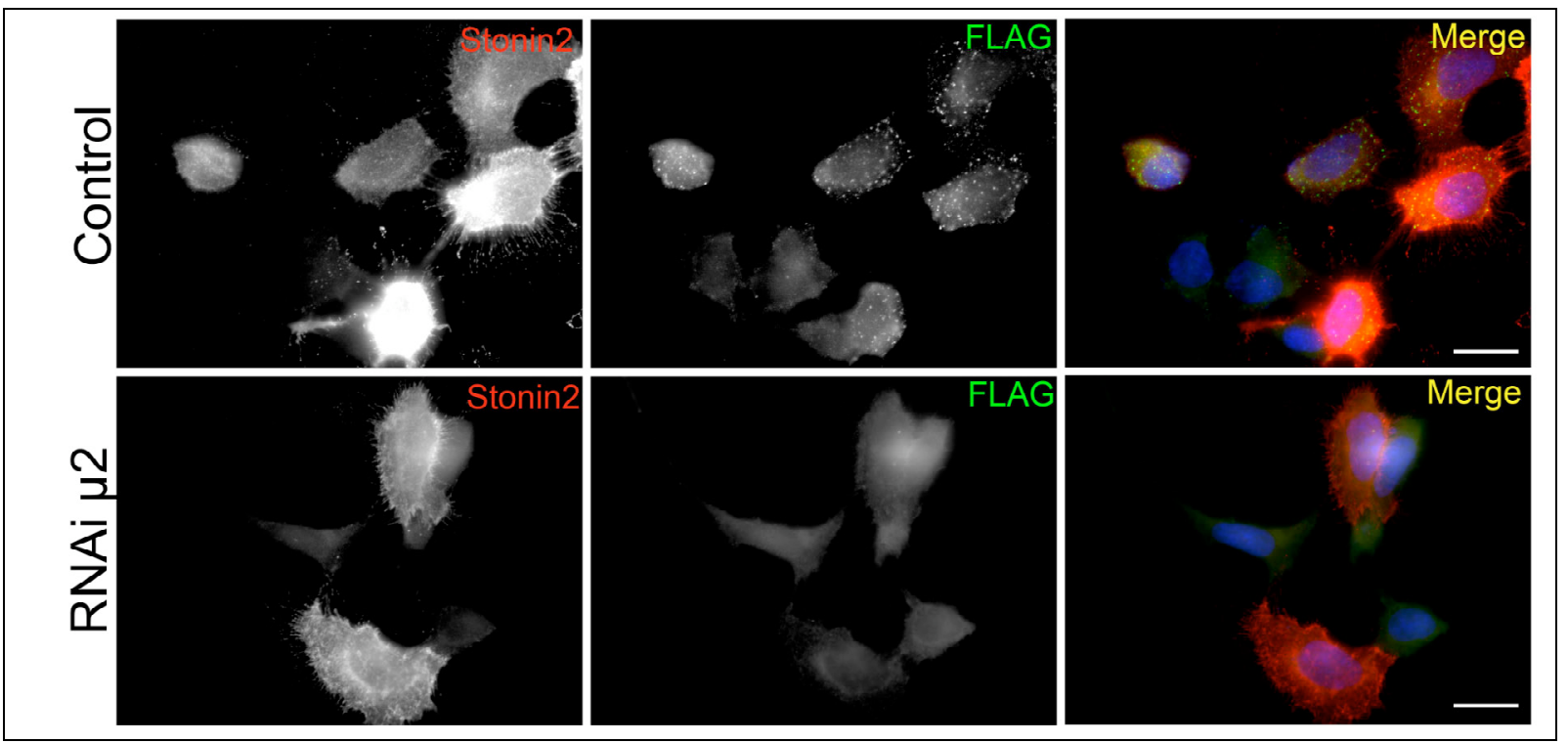

Figure 3.26 Stonin 2 cannot facilitate internalization of synaptotagmin 1 in the absence of AP2. HEK293-FLAG-Syt1 cells were transfected with stonin2 ${ }^{\text {WT }}$ in the presence or absence of $\mu 2$ siRNAs. An antibody uptake assay was performed essentially as described before however the cells were warmed up to room temperature for only 5 minutes for endocytosis to take place. The figure depicts cells expressing similar levels of stonin2. Cells that were not treated with $\mu 2$ siRNAs were able to internalize FLAG-synaptotagmin 1 into vesicular endosomal compartments. AP2 knock-down

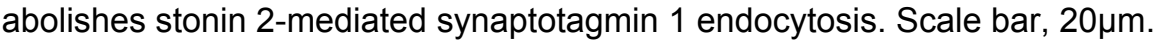


Even in the absence of exogenously expressed stonin 2, HEK293-FLAG-Syt1 cells were able to internalize minute amounts of synaptotagmin 1 when uptake assays were performed at $37^{\circ} \mathrm{C}$ for more than $1 \mathrm{~h}$ (Figure 3.22). Whether or not this is the result of expression of low levels of endonenous stonin 2 remains unclear. In agreement with the data shown above, we found that RNAi mediated knock-down of AP2 expression inhibits synaptotagmin 1 endocytosis in the absence of exogenously supplied stonin 2 (Figure 3.27). Unfortunately, siRNAs against stonin 2 were ineffective in silencing endogenous stonin 2. Therefore, we could not test whether the minimal synaptotagmin 1 endocytosis seen in HEK cells was dependent on endogenous stonin 2 .

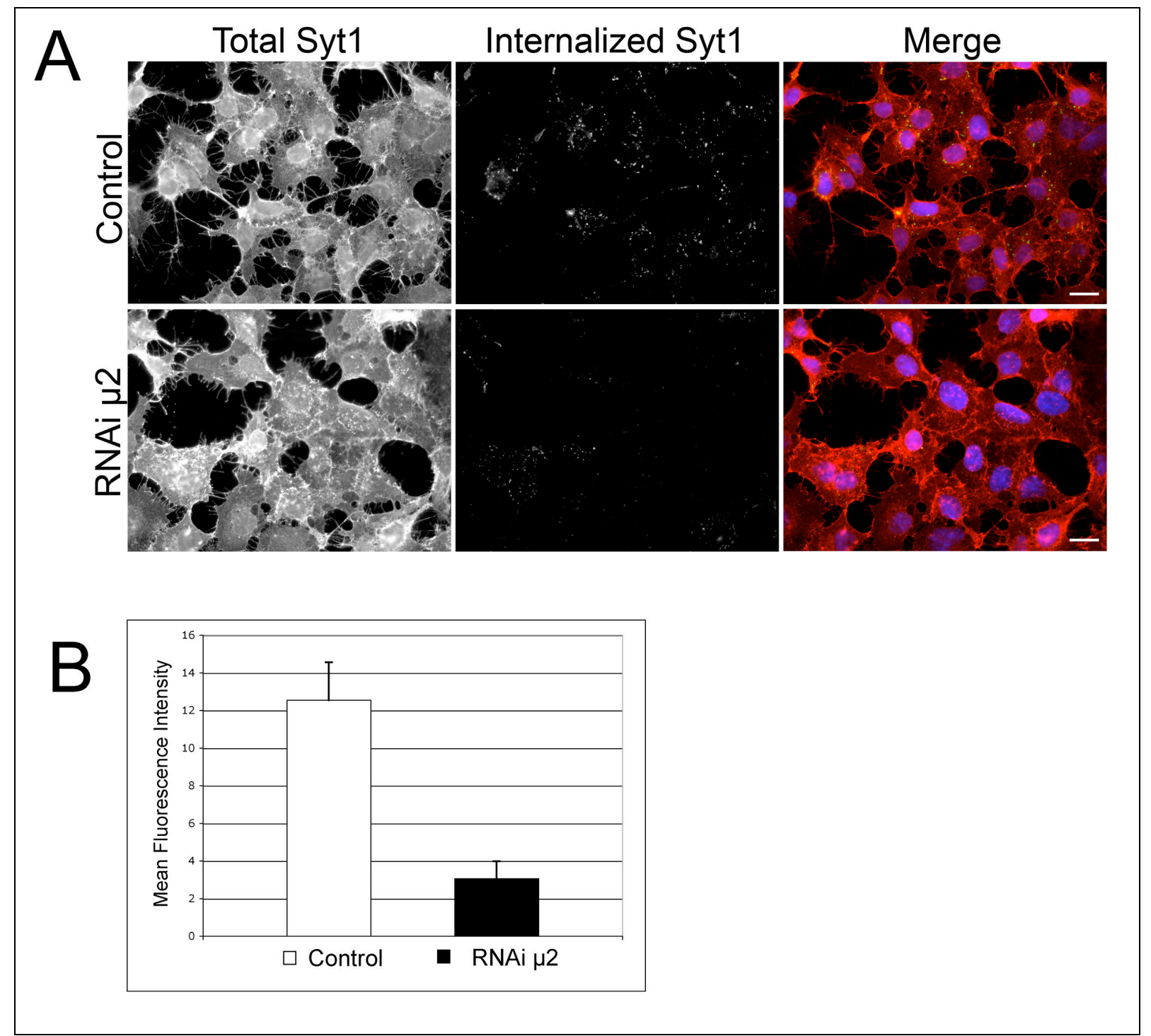

Figure 3.27 AP2 knock-down also inhibits endocytosis of synaptotagmin 1 in the absence of exogenously expressed stonin 2. (A) HEK293-FLAG-Syt1 cells display a limited ability to endocytose synaptotagmin 1 without stonin 2 transfection. However, siRNA-mediated knock down of AP2- $\mu 2$ inhibits also this minimal synaptotagmin 1 endocytosis. Scale bar, $20 \mu \mathrm{m}$. (B) Quantification of the data shown in (A). HEK293 cells stably expressing FLAG-synaptotagmin 1 were either mock- 
transfected or transfected with $\mu 2$ siRNAs $(2 \mathrm{x})$ and cultured for $96 \mathrm{~h}$ after the first transfection. Antibody uptake assays were performed for $2 \mathrm{~h}$ at $37^{\circ} \mathrm{C}$. Quantification of FLAG-synaptotagmin 1 internalization was done as described in the legend to figure 3.28 using nine data sets from three independent experiments. One of these data sets is shown in (A). Error bars represent standard deviations of these nine data sets.

Next, we decided to further analyze the structural requirements for stonin 2- $\mu \mathrm{HD}$ binding to synaptotagmin 1 and its role in endocytosis. The presented data up to this point indicate that stonin 2 might interact with synaptotagmin 1 with a higher affinity compared to $\mu 2$, although much of the evidence has been obtained by indirect experiments (i.e. endocytosis, colP assays). The $\mu \mathrm{HD}$ of stonin 2 and the cargo binding domain of AP2- $\mu 2$ share only a distant homology to each other (Figure 1.16). Especially noteworthy is the presence of two peptide stretches within the stonin 2$\mu \mathrm{HD}$ that are absent from $\mu 2$; residues 736-765 and residues 865-897. In order to gain initial insights into the synaptotagmin 1 interaction site within the stonin $2-\mu H D$, we created a set of deletion mutants.

We generated 4 truncation constructs of the carboxy-terminal domain of stonin 2 and a mutant of stonin 2 carrying an internal deletion (see Table 3.1). HEK293-FLAGSyt1 cells were transfected with these constructs and subjected to anti-FLAG antibody uptake as described before. Truncations 1 and 2 as well as the internal deletion mutant were all able to facilitate the internalization of synaptotagmin 1 (Figure 3.28). By contrast, truncations 3 and 4 were unable to facilitate synaptotagmin 1 uptake.

We thus conclude that, an intact stonin $2-\mu \mathrm{HD}$ is required for its ability to facilitate synaptotagmin 1 internalization but the exact interaction site remains unknown. 

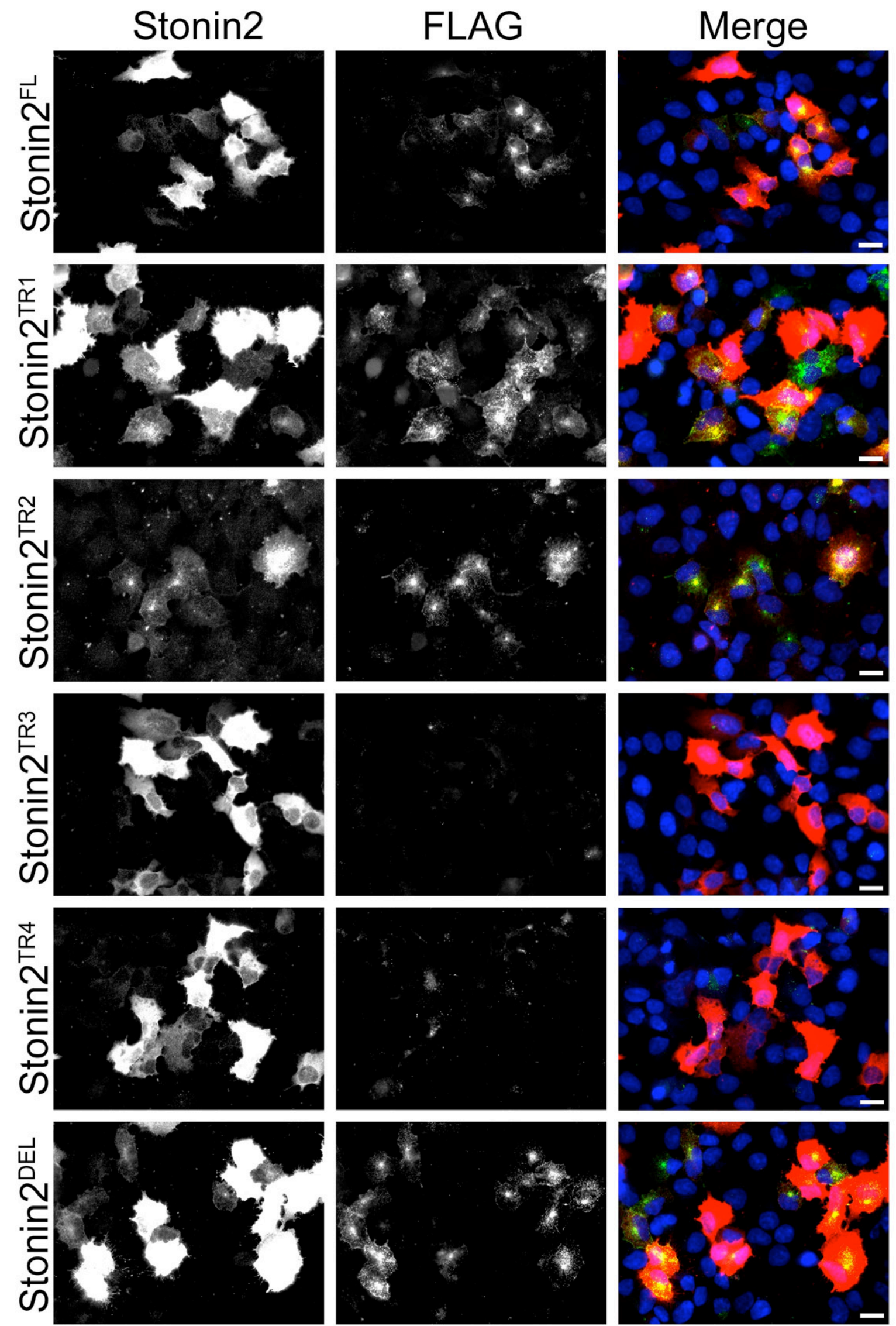

Figure 3.28 Stonin 2 carboxy-terminal truncations and synaptotagmin 1 endocytosis. HEK293FLAG-Syt 1 cells were transfected with the indicated stonin 2 constructs. $24 \mathrm{~h}$ post-transfection, antibody internalization assays were performed for $2 \mathrm{~h}$ at $37^{\circ} \mathrm{C}$. After fixation and surface antibody 
blocking, cells were immunostained for stonin 2 (red) and FLAG (green). Images were captured from samples exposed to the excitatory light for equal periods of time. Note that some cells expressing very low level of stonin 2 are equally competitive in internalizing synaptotagmin 1 as higher level expressing cells.

Almost all synaptagmin family proteins contain a conserved WHXL sequence motif in their carboxy-terminal domain which is a part of the C2B domain $\beta$-strands according to the crystal structure (Fernandez et al., 2001). Previously, this motif has been implicated in docking of synaptic vesicles to the presynaptic plasmalemma (Fukuda et al., 2000), and AP2-mediated endocytosis of synaptotagmin 1 from overexpressing PC12 cells (Jarousse et al., 2003).

Tyrosine based sorting signals are recognized by a mainly hydrophobic pocket within the $\mu 2$ cargo binding domain. Several amino acid residues that were shown to be

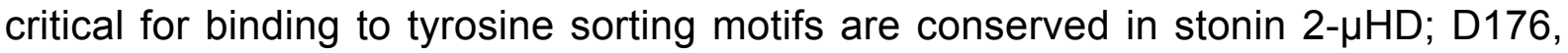
K420 and W421. Since WHXL motifs would be structurally similar to YXXØ motifs, we hypothesized that stonin 2 might be recognizing synaptotagmin 1 by a mechamism analogous to tyrosine motif binding to AP2- $\mu$. However, triple mutation of these residues to alanines did not have any negative effect on synaptotagmin 1 internalization, even when the internalization assays were carried out for 5 min at RT (data not shown, see Table 3.1).

We conclude that the determination of the exact synaptotagmin 1 interaction site within stonin $2-\mu \mathrm{HD}$ may require a structural analysis involving purified protein domains.

\begin{tabular}{|c|c|c|}
\hline Construct & $\begin{array}{c}\text { Syt1 } \\
\text { uptake }\end{array}$ & Comments \\
\hline Stonin2 ${ }^{\mathrm{WT}}$ & +++ & Wild type, full length \\
\hline Stonin $2^{\partial W W W}$ & + & All $W V X F \Rightarrow A \vee X F$ \\
\hline Stonin2 $2^{\partial W F}$ & + & All $W V X F \Rightarrow A V X A$ \\
\hline Stonin $2^{\partial W F \partial N P F}$ & - & All $W V X F \Rightarrow A V X A$ and 2 NPF $\Rightarrow N A V$ \\
\hline Stonin2 $2^{2335}$ & - & N-terminal 335 a.a. deleted, lacks WVXF and NPF motifs \\
\hline Stonin $2^{\partial 420}$ & - & N-terminal 420 a.a. deleted, lacks the whole Ser/Pro rich domain \\
\hline Stonin $2^{\mathrm{NT}}$ & - & $\mathrm{N}$-terminal 1-555 a.a. , $\mu \mathrm{HD}$ deleted \\
\hline Stonin $2^{\mathrm{NT}}-\mu 2$ & - & Chimera between stonin2-NT and $\mu 2-\mathrm{CT}$ cargo binding domain \\
\hline Stonin2 ${ }^{\mathrm{TR} 1}$ & +++ & C-terminal truncation, 887 a.a. long \\
\hline Stonin $2^{T R 2}$ & +++ & C-terminal truncation, 875 a.a. long \\
\hline Stonin $2^{T R 3}$ & - & C-terminal truncation, 864 a.a. long \\
\hline Stonin $2^{T R 4}$ & - & C-terminal truncation, 840 a.a. long \\
\hline Stonin $2^{\mathrm{DEL}}$ & +++ & C-terminal deletion, $\partial 736-765,30$ residue stretch \\
\hline Stonin $2^{\star T y r}$ & +++ & Point mutation of putative Tyr binding pocket D574A, KW860AA \\
\hline Stonin1 & - & Full length, wild type, pcHA or EGFP tagged stonin1 \\
\hline
\end{tabular}

Table 3.1 Effect of different stonin constructs on synaptotagmin1 endocytosis. 


\subsection{Sorting of synaptotagmin 1 following endocytosis}

Following the synaptic vesicle fusion-event in presynaptic nerve terminals, synaptotagmin 1 is endocytosed and incorporated into synaptic vesicles with or without traversing through an endosomal compartment. Apart from neuronal synaptic vesicles, synaptotagmin 1 is also found on synaptic-like microvesicles (SLMV) and dense core vesicles in secretory endocrine cells such as pituitary cells, adrenal chromaffin cells (or PC12 cells), and pancreatic $\beta$-cell lines (Fukuda, 2003).

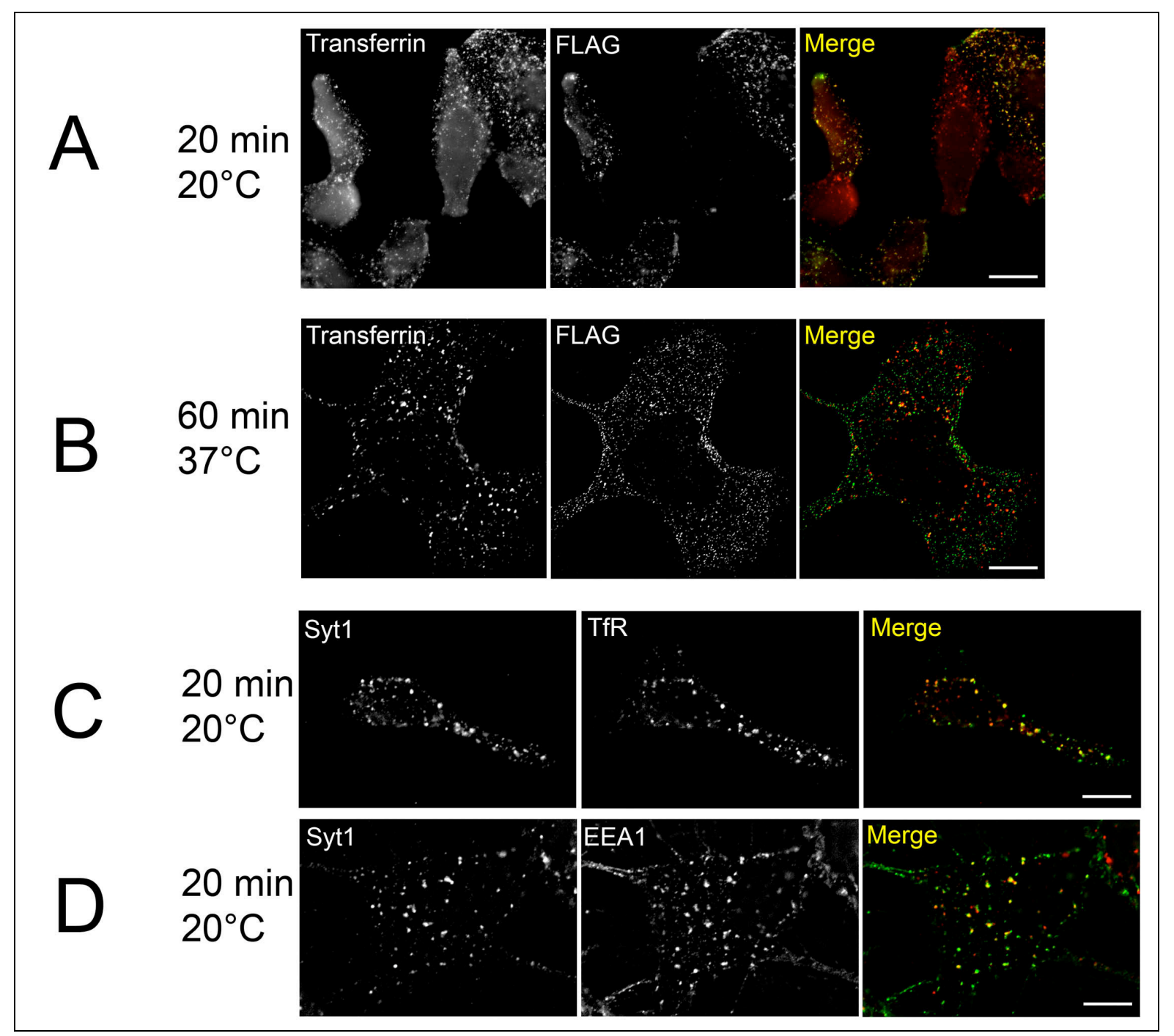

Figure 3.29 Internalized synaptotagmin 1 is sorted to early endosomes, from where it is trafficked to distinct perinuclear vesicles. (A) HEK293 cells stably expressing FLAG-synaptotagmin 1 and transiently transfected with stonin $2^{\text {WT }}$ were serum-starved for $2 \mathrm{~h}$ in OPTIMEM. Cells were incubated with $20 \mu \mathrm{g} / \mathrm{ml}$ Alexa ${ }^{594}$ labeled transferrin together with $\alpha-F L A G$ antibodies for 30 min on ice. Samples were warmed to $20^{\circ} \mathrm{C}$ for $20 \mathrm{~min}$ to allow for internalization. Endocytosed synaptotagmin 1

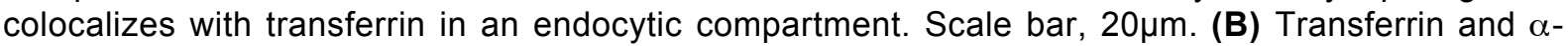
FLAG antibody co-internalization assays were performed as in $\mathbf{A}$, except that cells were incubated for $1 \mathrm{~h}$ at $37^{\circ} \mathrm{C}$. Transferrin and endocytosed synaptotagmin 1 localize to distinct vesicular compartments. Images were obtained by constrained iterative deconvolution of serial planes along the z-axis. Scale bar, $10 \mu \mathrm{m}$. (C) As described in the legend to $\mathbf{A}$ and $\mathbf{B}$, antibody uptake experiments were performed with HEK293 cells stably expressing FLAG-synaptotagmin 1 together with transiently transfected 
stonin 2. Instead of FLAG antibodies, a polyclonal rabbit antibody against the lumenal domain of synaptotagmin 1 was used (89.07). After 20 min internalization at $20^{\circ} \mathrm{C}$, cells were fixed, surface staining was blocked with goat-anti-rabbit IgG, and permeabilized cells were immunostained with $\alpha$ TfR antibodies. Endocytosed synaptotagmin 1 colocalizes with the TfR in early endocytic compartments. Scale bar, $20 \mu \mathrm{m}$. (D) Antibody uptake assays were perfomed as described in C. Following fixation and permeabilization, cells were stained with EEA1 antibodies. EEA1 and endocytosed synaptotagmin 1 colocalize in early endosomes. Scale bar, $20 \mu \mathrm{m}$.

HEK293 cells are fibroblast-like cells that do not express synaptotagmin 1 endogenously. There is also no compartment analogous to synaptic vesicles or other synaptotagmin 1 containing vesicular compartments. Therefore we were interested in analyzing the sorting pathway traversed by endocytosed synaptotagmin 1 and the nature of the perinuclear scattered vesicular compartments where it eventually accumulates. Plasmalemma-stranded synaptotagmin 1 in HEK293-FLAG-Syt1 cells, localized almost entirely to perinuclear vesicles upon stonin 2 overexpression. It is also possible to see stonin 2 colocalizing with synaptotagmin 1 on the same vesicles in cells expressing low levels of stonin 2 (data not shown).

First, we compared synaptotagmin 1 endocytosis to that of ligand-bound transferrin receptor which harbors a tyrosine based sorting signal in its carboxy-terminal cytosolic tail and can interact directly with AP2. Serum-starved HEK293-FLAG-Syt1 cells were labeled by incubating with anti-FLAG antibodies and Alexa ${ }^{594}$ labeled human serum transferrin on ice. Cells were brough up to RT and incubated for an additional $20 \mathrm{~min}$ for endocytosis to take place. Internalized synaptotagmin 1 colocalized with transferrin within enlarged endosomes scattered throughout the cell (Figure 3.29A). Double immunostaining of cells for synaptotagmin 1 and transferrin receptor (TfR) or EEA1, after 20 min of internalization at $\mathrm{RT}$ revealed a large degree of colocalization with both proteins (Figure 3.29C and D). Synaptotagmin 1 colocalized with TfR and EEA1 already after 5 minutes of internalization and segregated to different compartments after $1 \mathrm{~h}$ incubation at $37^{\circ} \mathrm{C}$ (data not presented). However, following incubation of cells at $37^{\circ} \mathrm{C}$ for $1 \mathrm{~h}$, resulted in a distinct intracellular distribution of synaptotagmin 1 different from transferrin positive early recycling endosomes. Instead, synaptotagmin 1 localized to small vesicles concentrated around a spot near the cell nucleus, which were distinct from transferrin-positive compartments (Figure 3.29B).

After $2 \mathrm{~h}$ of internalization at $37^{\circ} \mathrm{C}$, a large portion of synaptotagmin 1-positive perinuclear vesicles also contained AP2 but not clathrin (Figure 3.30). Considering the robust interaction between stonin 2 and AP2 via WVXF motifs, this finding is not 
surprising. Colocalization of AP2 with internalized cargo proteins has also been demonstrated for internalized EGF receptor (Sorkina et al., 1999).

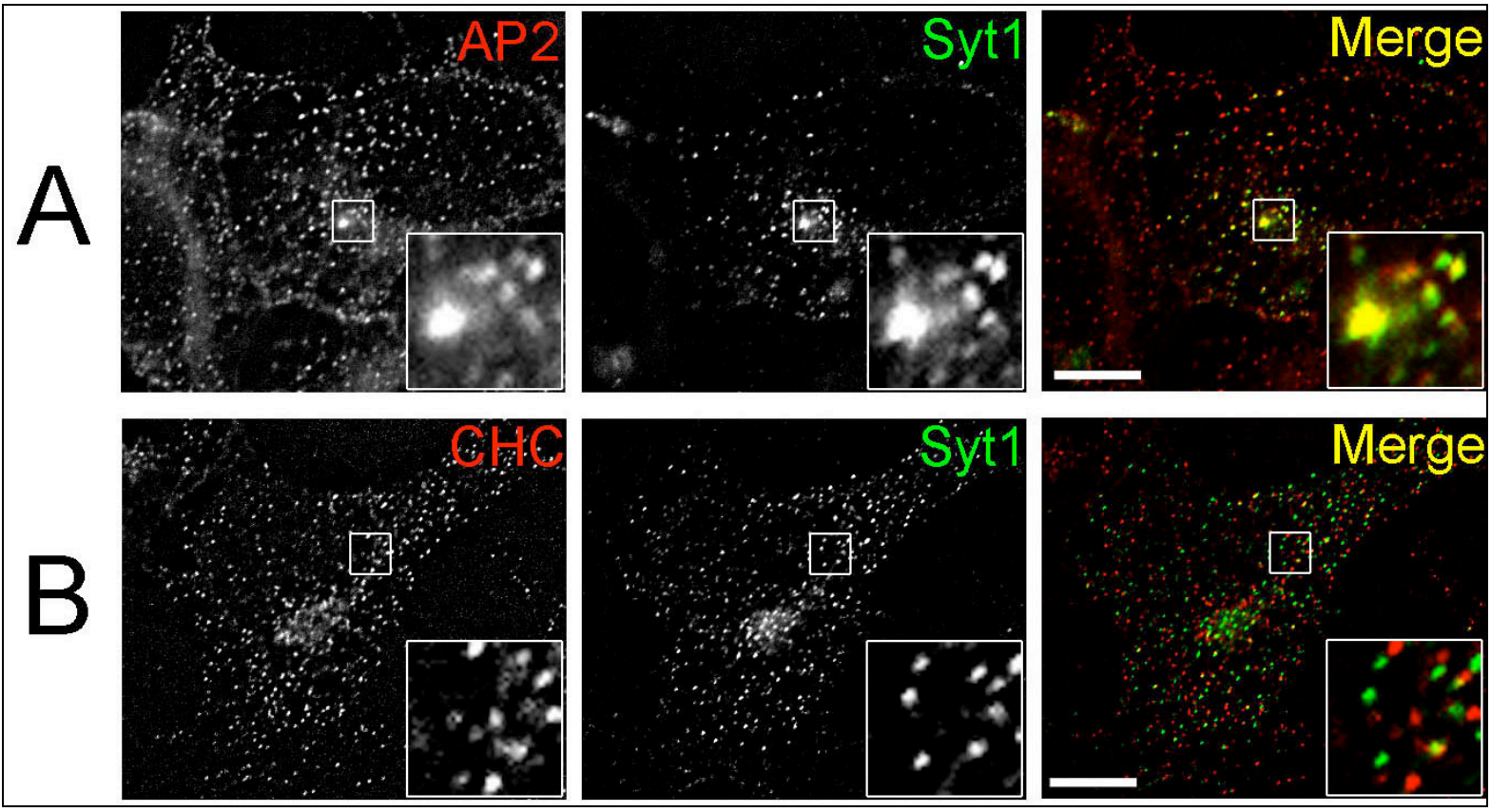

Figure 3.30 Colocalization of internalized synaptotagmin 1 with AP2. HEK293-FLAG-Syt1 cells were incubated with polyclonal antibodies (batch 89.07) against synaptotagmin 1 lumenal domain and let internalize for $2 \mathrm{~h}$. After blocking of the surface antibody, cells were immunostained for synaptotagmin1 (green) and (A) AP2 or (B) clathrin (red). Internalized synaptotagmin 1 partially colocalizes with AP2 but not clathrin on perinuclear endosomes. Images were generated by deconvolution microscopy. Insets in the bottom right corner show 4 times magnification of the depicted areas. Scale bar, $5 \mu \mathrm{m}$.

Finally, we have analyzed stonin 2-mediated endocytosis and sorting of synaptotagmin 1 in PC12, a cell line derived from neuroendocrine chromaffin cells. Chromaffin cells express both stonin 2 and synaptotagmin 1 endogenously. Synaptotagmin 1 is present within both large dense core vesicles (LDCV) and SLMVs. PC12 cells also express stonin 2 and synaptotagmin 1 according to WB analysis albeit lower levels (Figure 3.3).

The majority of lumenally FLAG-tagged synaptotagmin 1 became stranded at the plasma membrane of PC12 cells upon overexpression. As in HEK293 cells, coexpression of stonin 2 facilitated endocytosis of synaptotagmin 1 more than 10 fold (data not shown). In order to determine the identity of the vesicles where synaptotagmin 1 was targeted to following stonin 2 facilitated endocytosis and sorting, we cotransfected the cells with either synaptopHlourin (a pH-sensitive variant of EGFP fused to the lumenal domain of synaptobrevin 2; Miesenbock et al., 1998) or mRFP tagged synaptophysin. Both synaptophysin and synaptobrevin 2 are contained within SLMVs in PC12 cells, but the latter is also present on LDCVs. 


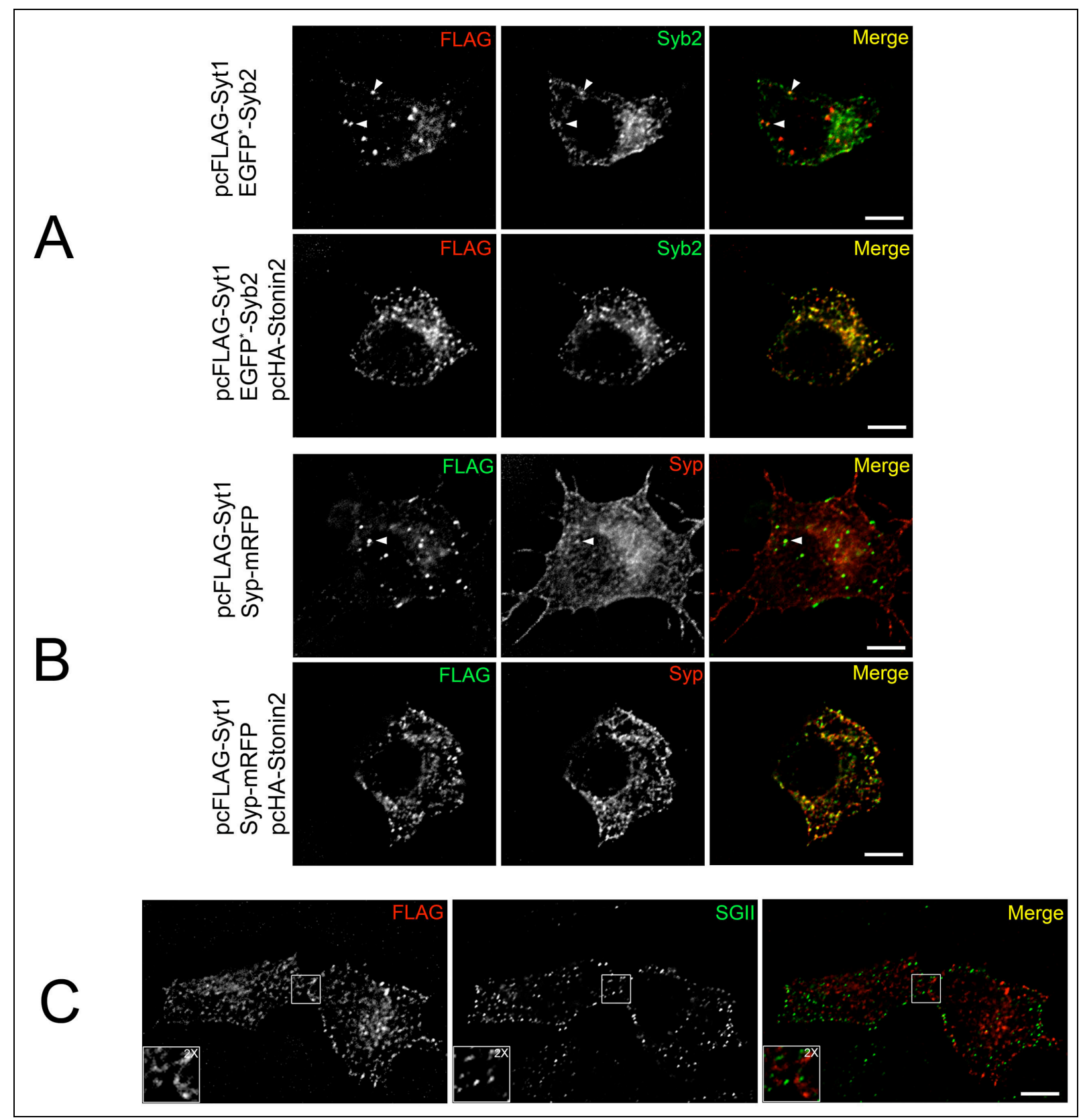

Figure 3.31 Stonin 2 facilitates endocytic internalization of synaptotagmin 1 and its sorting to SLMVs in neuroendocrine cells. (A) PC12 cells were cotransfected with synaptotagmin 1 and EGFP*-synaptobrevin 2 constructs (synaptopHlourin) in the presence or absence of stonin 2. Antibody uptake assays were performed for $2 \mathrm{~h}$ at $37^{\circ} \mathrm{C}$ essentially as described before. Stonin 2 expression facilitates synaptotagmin 1endocytosis and sorting to synaptopHluorin (EGFP*-synaptobrevin 2)containing SLMVs. In the absence of transfected stonin 2, endocytosed synaptotagmin 1 displays only limited colocalization with synaptopHluorin (white arrowheads). Scale bar, $5 \mu \mathrm{m}$. (B) As in A, except that PC12 cells were transfected with a synaptophysin-mRFP construct which partially localizes to SLMVs and to the plasma membrane in the absence of transfected stonin 2. Endocytosed synaptotagmin 1 colocalizes with synaptophysin to SLMVs only in the presence of stonin 2 . Scale bar, $5 \mu \mathrm{m}$. (C) Internalized synaptotagmin1 does not colocalize with the DCV marker secretograninll in neuroendocrine cells. PC12 cells were cotransfected with synaptotagmin 1 and stonin 2 constructs. Antibody uptake assays were performed as before. Cells were stained post-fixation with polyclonal antisera against the DCV marker secretogranin II. Scale bar, $5 \mu \mathrm{m}$. All images were generated by constrained iterative deconvolution fluorescence microscopy. 
After two hours of incubation at $37^{\circ} \mathrm{C}$, internalized synaptotagmin 1 largely colocalized with both synaptobrevin 2 and synaptophysin in SLMVs (Figure 3.31). In the absence of stonin 2, synaptotagmin 1 internalization was inefficient (note that images showing synaptotagmin 1 internalization in the absence of stonin 2 were obtained by five times longer exposure) and internalized synaptotagmin 1 was targeted to large vesicles which only partially colocalized with synaptobrevin 2 or synaptophysin (arrowheads). Overexpression of stonin 2 also resulted in recovery of otherwise partially membrane stranded synaptophysin-mRFP (Figure 3.31B).

Double immunostaining of stonin 2/synaptotagmin 1 cotransfected PC12 cells after 2 $\mathrm{h}$ of antibody uptake showed that synaptotagmin 1 and a LDCV marker secretograninll did not colocalize (Figure 3.31C). Therefore, we conclude that stonin 2 facilitates sorting of endocytosed synaptotagmin 1 selectively to SLMVs in PC12 cells.

In summary, we hypothesize that stonin 2 is an AP2-dependent sorting adaptor for synaptotagmin 1 internalization and endocytic sorting to neurosecretory vesicles.

\subsection{Analysis of stonin 2 deficient mice}

Analysis of protein function by targeted deletion or mutagenesis of the encoding genes in mice has become a common experimental procedure in the last two decades. Sequencing of the mouse genome and availability of mouse embryonic stem cell lines have made gene manipulations easy (Bockamp et al., 2002; van der Weyden et al., 2002). Generation of knock-out and knock-in mice strains has also become a standard approach for scientists studying synaptic vesicles, their biogenesis, exocytosis, endocytosis and recycling. Analysis of transgenic mice models lacking synaptojanin 1 (Cremona et al., 1999), PIPKI $\gamma$, (Di Paolo et al., 2004), amphiphysin (Di Paolo et al., 2002), synaptotagmin 1 (Geppert et al., 1994) and synaptobrevin 2 (Schoch et al., 2001; Deak et al., 2004) have been valuable in providing insight into these proteins' functions.

In order to achieve a better understanding of stonin 2 functions in synaptic vesicle endocytosis and recycling, we have decided to produce a stonin 2 deficient mouse strain by conventional gene knock-out procedures. The generation of stonin 2 deficient mice is explained in detail in sections 2.2.10 to 2.2.13. 


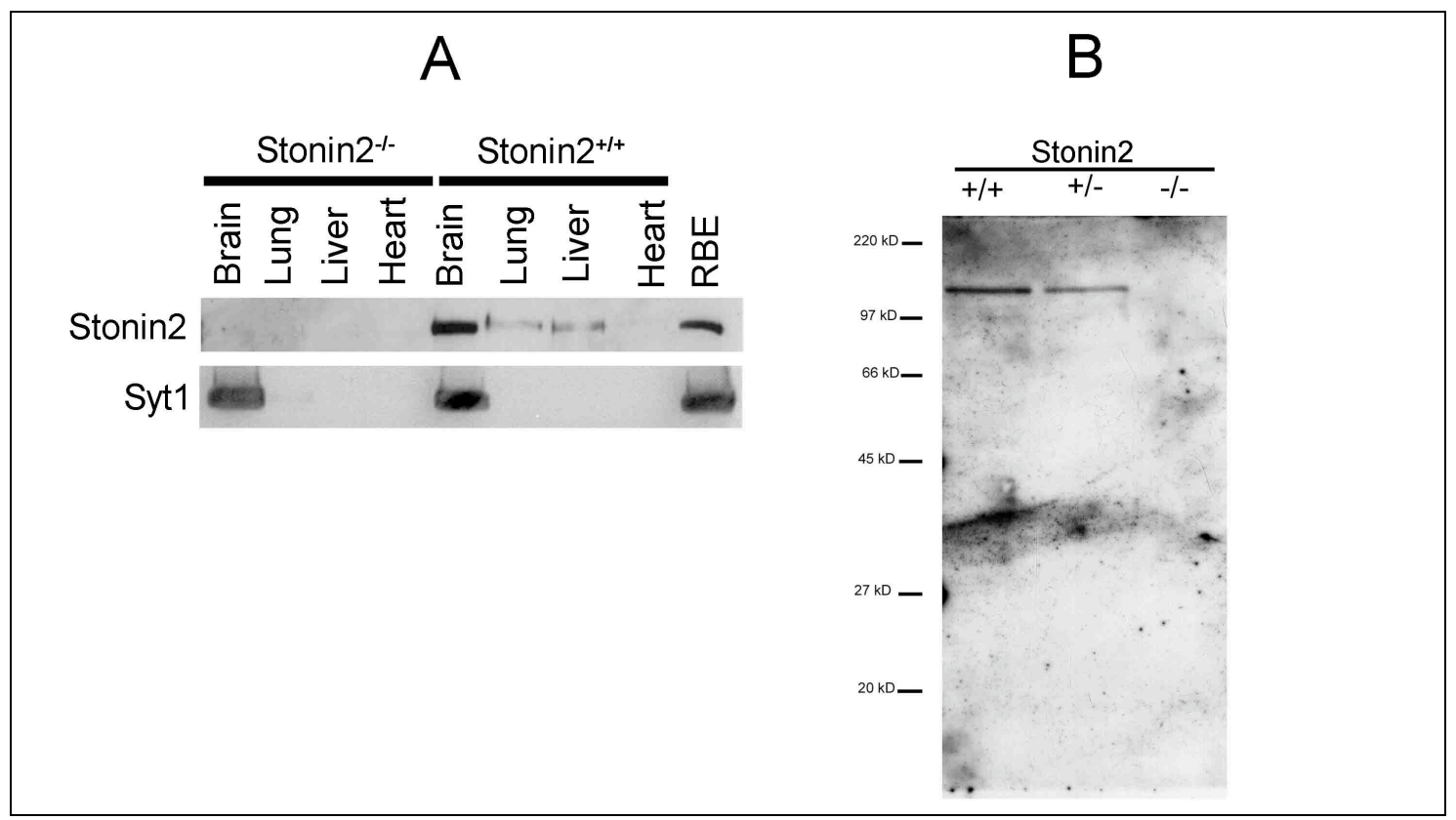

Figure 3.32 Western blot analysis of stonin 2 knock-out. (A) Equal amounts of protein extracts from indicated tissues of wild type and knock-out animals were separated by $8 \%$ SDS-PAGE and analyzed by WB using stonin 2 and synaptotagmin 1 antibodies. Rat brain extract (last lane) has been loaded as a control. (B) Equal amounts of brain extracts from stonin 2 wild type, heterozygous and knock-out animals were separated by a $12 \%$ SDS gel and analyzed by immunoblotting with stonin 2 antibodies. Targeting strategy does not cause expression of a shorter stonin 2 harboring aminoterminal 200 amino acids as this would be detected by stonin 2 antibodies raised against a stonin 2NT(1-555) construct.

Targeted disruption of exon 4 of the stonin 2 gene by replacement with a neomycin resistance cassette resulted in disappearance of the 120-130kDa protein band recognized by anti-stonin 2 antibodies in WBs from all tissues tested (Figure 3.32A). Our targeting strategy did not involve the deletion of the first three coding exons or any possible unidentified exons in the 5' side of the gene. Usually deletion of one internal exon results in total loss of protein expression as the mRNA or the truncated protein become unstable. However, there is a low risk of expression of a truncated mRNA that could be translated into a truncated stonin 2 protein containing the first 200 residues. Such a protein could have unpredicted physiological effects as it would harbor two WVXF motifs. During the construction of the stonin 2 targeting vector, these motifs were not yet identified. In order to check for the expression of truncated stonin 2 variants in these mice, we separated brain extracts from these animals using a higher percentage gel and probed the blots with polyclonal stonin 2 antibodies raised against the amino-terminal 555 amino acids. We were unable to detect any protein bands indicative of the expression of a truncated stonin 2 (Figure 3.32B). Therefore, we conclude that we have created a bona fide stonin 2 knock-out mouse line. 
Preliminary analysis of the animals showed that stonin 2 knock-out mice were viable and fertile. They showed no noticeable physical or behavioral abnormalities. Stonin 2 deficient females were indistinguishable from their wild-type litter mates with regard to mothering behaviour.

In order to further characterize the viability of stonin 2 knock-out mice, 9 pairs of heterozygous males and females were crossed with each other and their progeny were closely observed for 3 weeks. Genotyping of the surviving offsprings at the end of three weeks showed a roughly Mendelian distribution of the progeny. The ratio of stonin $2^{+/+}$: stonin $2^{+/-}:$stonin $2^{-/-}$animals was $0.29: 0.49: 0.22$ (Table 3.2). Although the number of stonin $2^{-/-}$offspring seemed to be less than that anticipated from a perfect Mendelian segregation of chromosomes, this deviation was not statistically significant. According to the Chi-Square test, such a distribution has a probability of occurrence between $50 \%$ to $70 \%$.

It is a well established fact that the genetic background of mice can influence the resulting phenotype. A particular mutation can cause a severe phenotype in one background and be less severe in another. It is usually advisable to test the effects of the mutation on phenotype in a pure genetic background. In the crossing experiment explained above and all other experiments described below, the animals used had a mixed background of 129/ola and C57/BL6 with roughly equal contributions. This will result in progeny with different genetic backgrounds. To overcome this problem, we generated a congenic mouse strain, by outcrossing stonin 2 animals to nmri mice 5 times. These viable and fertile animals, (more than 95\% nmri background), will be used for further analysis of stonin 2 deficiency.

\begin{tabular}{|c|c|c|c|c|c|c|c|c|c|c|c|}
\hline CROSS & $\mathbf{1}$ & 2 & 3 & 4 & 5 & $\mathbf{6}$ & $\mathbf{7}$ & $\mathbf{8}$ & 9 & TOTAL & $\%$ \\
\hline$+/+$ & 7 & 10 & 14 & 7 & 7 & 3 & 1 & 1 & 2 & 52 & 29 \\
\hline$+/-$ & 14 & 13 & 19 & 11 & 8 & 7 & 8 & 8 & 2 & 90 & 49 \\
\hline$-/-$ & 11 & 7 & 9 & 5 & 3 & 3 & 1 & 1 & 0 & 40 & 22 \\
\hline Total & 32 & 30 & 42 & 23 & 18 & 13 & 10 & 10 & 4 & 182 & 100 \\
\hline
\end{tabular}

Table 3.2 Genotypes of the offspring as a result of stonin 2 heterozygote crosses.

The Drosophila stoned mutation causes mislocalization and degradation of synaptotagmin 1 . There is a reduction of synaptotagmin 1 expression level in the 
larvae due to degradation and this could be detected by western blotting (Fergestad et al., 1999). Therefore, we compared the expression levels of synaptotagmin 1 as well as that of several other endocytic proteins in total protein extracts of brains derived from stonin 2 knock-out and wild-type animals. However, stonin 2 deficiency did not cause any gross changes in the levels of the proteins tested assayed by quantitative western blotting (Figure 3.33).

In addition to degradation, lethal stoned mutations cause a selective mislocalization of synaptotagmin 1 from synaptic vesicle clusters to the presynaptic plasma membrane in neuromuscular junctions of the Drosophila embryo (Fergestad et al., 1999; Stimson et al., 2001). This could result from inefficient endocytosis of synaptotagmin 1 in the absence of stoned proteins, especially stoned B (Estes et al., 2003). One possible effect of stonin 2 deficiency in mice could be the presence of increased quantities of synaptotagmin 1 stranded on the presynaptic plasma membrane. Hence, we analyzed the distributions of synaptotagmin 1 together with other endocytic and presynaptic proteins in different membrane fractions obtained from the brains of wild-type and knock-out animals.

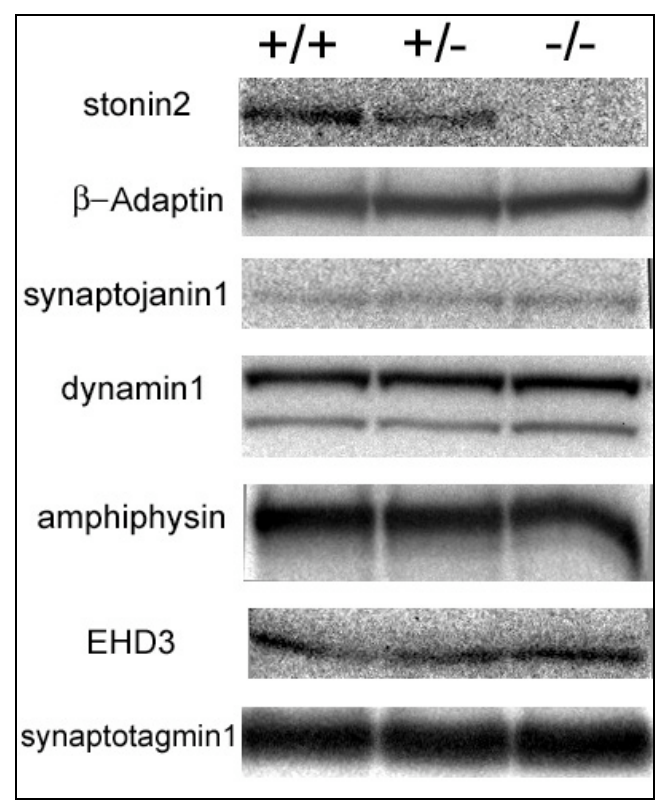

Figure 3.33 Quantitative western blot analysis of protein expression levels in stonin 2 animals. Equal amounts of brain protein extracts from different stonin 2 genotype animals were separated by SDS-PAGE and western blotted. Detection and quantification was performed by $\left[{ }^{125}\right.$ I]Protein A labeling values not shown). No significant difference in the intensities of the tested proteins was found, excepting stonin 2 .

As reported before, stonin 2 was enriched in the LP2 fraction which contains small membrane bound vesicles including synaptic vesicles and clathrin-coated vesicles, 
compared to the cytosolic pool (Walther et al., 2001 and 2004). Synaptotagmin 1 and synaptophysin, synaptic vesicle proteins, were enriched in the LP2 fraction, whereas synaptotagmin 7, a presynaptic plasma membrane protein (Sugita et al., 2001) was slightly enriched in the LP1 fraction.

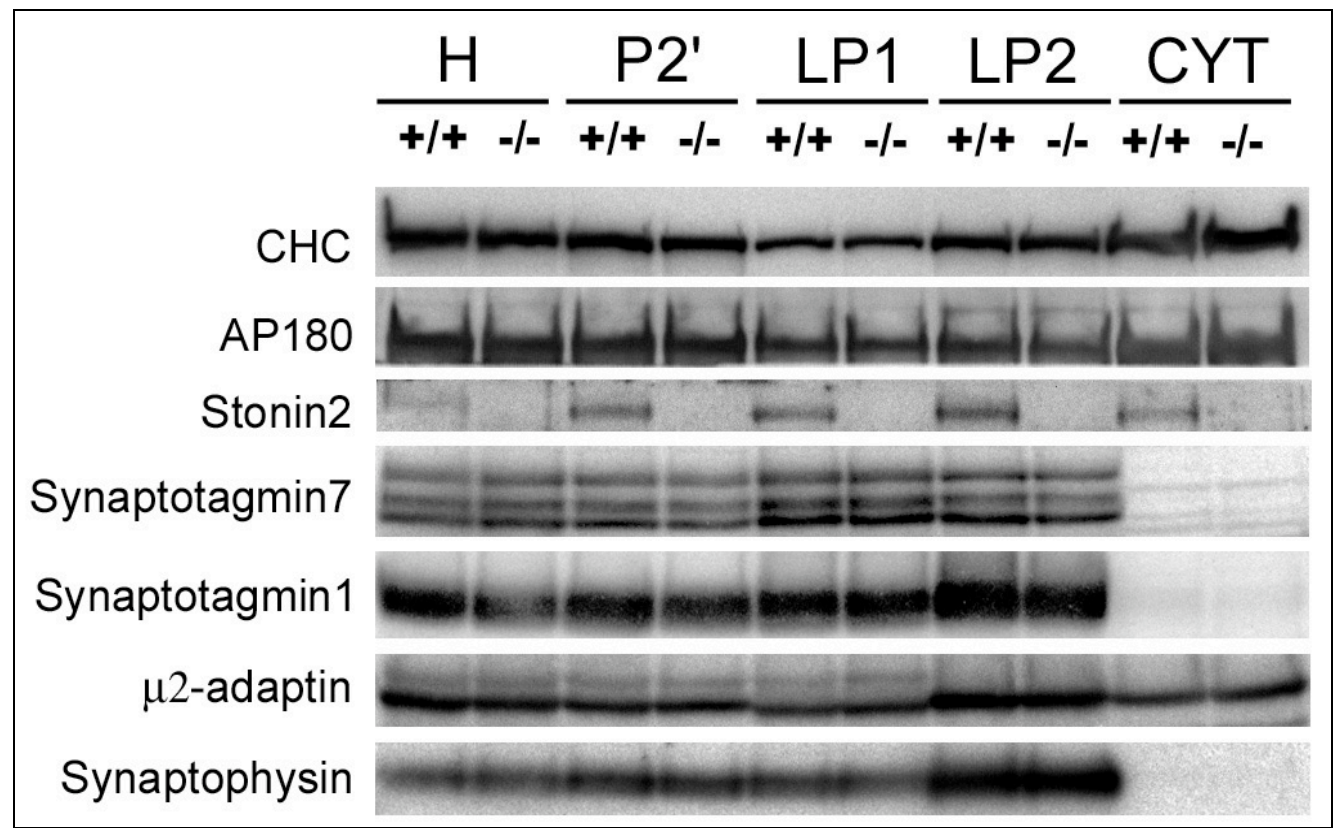

Figure 3.34 Subcellular distribution of synaptotagmin 1 and other proteins in wild-type and knock-out mice brain extracts. Membrane fractions H (homogenate), P2' (washed synaptosomes), LP1 (Lysed pellet 1, synaptosomal plasma membranes), LP2 (Lysed pellet 2, clathrin coated vesicles and synaptic vesicles) and CYT (synaptosomal cytosol) were prepared according to a modification of the protocol by Maycox et al., 1992. 5 brains from 6-8 weeks old adult mice from each genotype were combined to prepare the fractions. Equal amounts of protein were separated by $8 \%$ SDS-PAGE and immunoblotted using quantitative $\left[{ }^{125} \mathrm{I}\right]$ protein $\mathrm{A}$ detection system.

In contrast to the phenotype seen in Drosophila, we could not detect any significantly different subcellular distribution of synaptotagmin 1 in the stonin 2 knock-out animals. The distribution of synaptotagmin 1 between LP1 and LP2 fractions in knock-out animal brains, was similar to that of wild-type animals (Figure 3.34). There was also no difference with respect to AP2 distribution (detected by immunobloting against AP2- $\mu 2$ ) or other synaptic proteins such as $\mathrm{CHC}, \mathrm{AP} 180$, synaptotagmin 7 or synaptophysin.

Biochemical experiments, as described above, may not be sensitive enough to detect minor alterations in synapse morphology or synaptotagmin 1 localization. Therefore, we cultured hippocampal neurons obtained from wild-type or knock-out neonatal mice and analyzed them by immunofluorescence microscopy. Density or morphology of the synapses (visualized by synaptophysin or synaptotagmin 1 immunostaining) 
did not show any noticable difference. Synaptotagmin 1 immunostaining in the knockout neurons was indistinguishable from that seen in the wild-type (Figure 3.35).

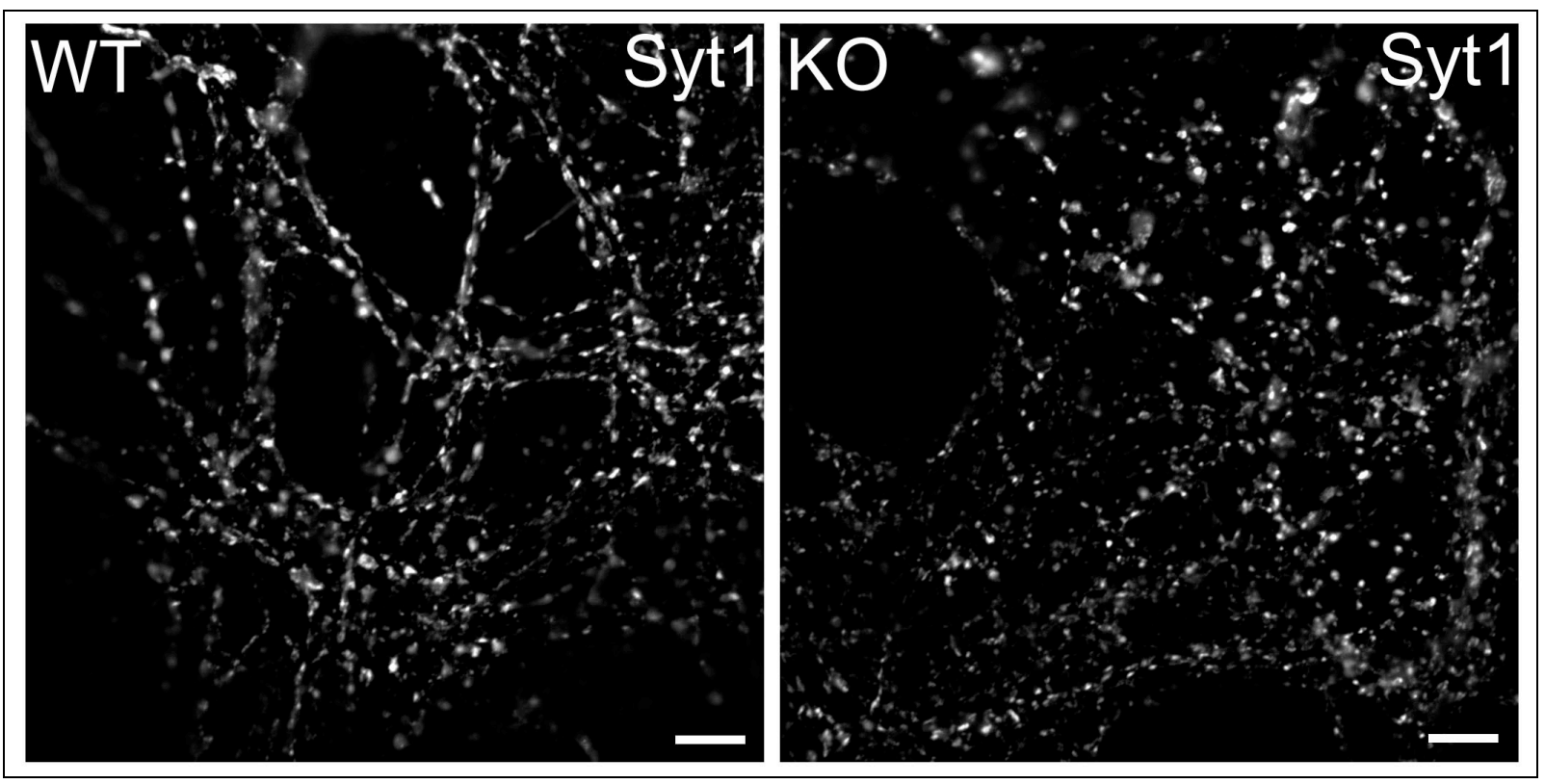

Figure 3.35 Synaptotagmin 1 immunostaining and localization in stonin 2 wild-type and knockout neurons. Hippocampal neurons from newly born pups were isolated and cultured together with glial cells for 2 weeks. Cells were fixed with 4\% PFA and immunostained for stonin 2 (not shown) and synaptotagmin 1 antibodies. In stonin 2 knock-out neurons no defect in synaptogenesis, synapse density or synaptotagmin 1 localization could be observed compared to wild-type. Scale bar, $10 \mu \mathrm{m}$.

Preliminary electron microscopic analysis of brain sections (performed in collaboration with Dr. G. Ahnert-Hilger) did not reveal any major changes in the number of vesicles per bouton, the membrane of docked vesicles or any obvious morphological alterations within the presynaptic compartment (data not shown).

As described before (section 3.2), stonin 2 partially colocalized with synaptotagmin 1 at presynaptic vesicle clusters in a subpopulation of mature cortical neurons cultured in vitro. Somewhat surprisingly stonin 2 expression appeared to be very low in immature neurons or neurons derived from differentiated embryonic stem cells (Figure 3.36).

In addition, we noticed that in hippocampal neuron/glial mixed cultures the underlying glial cells displayed a punctate pattern of stonin 2 immunoreactivity that in many cases appeared to be much stronger than the staining seen in neurons attached to top of the glial cell lawn. This glial cell staning was specific for stonin 2 as it was absent in neuron/glial cultures derived from stonin 2 knock-out animals (Figure 3.37). 


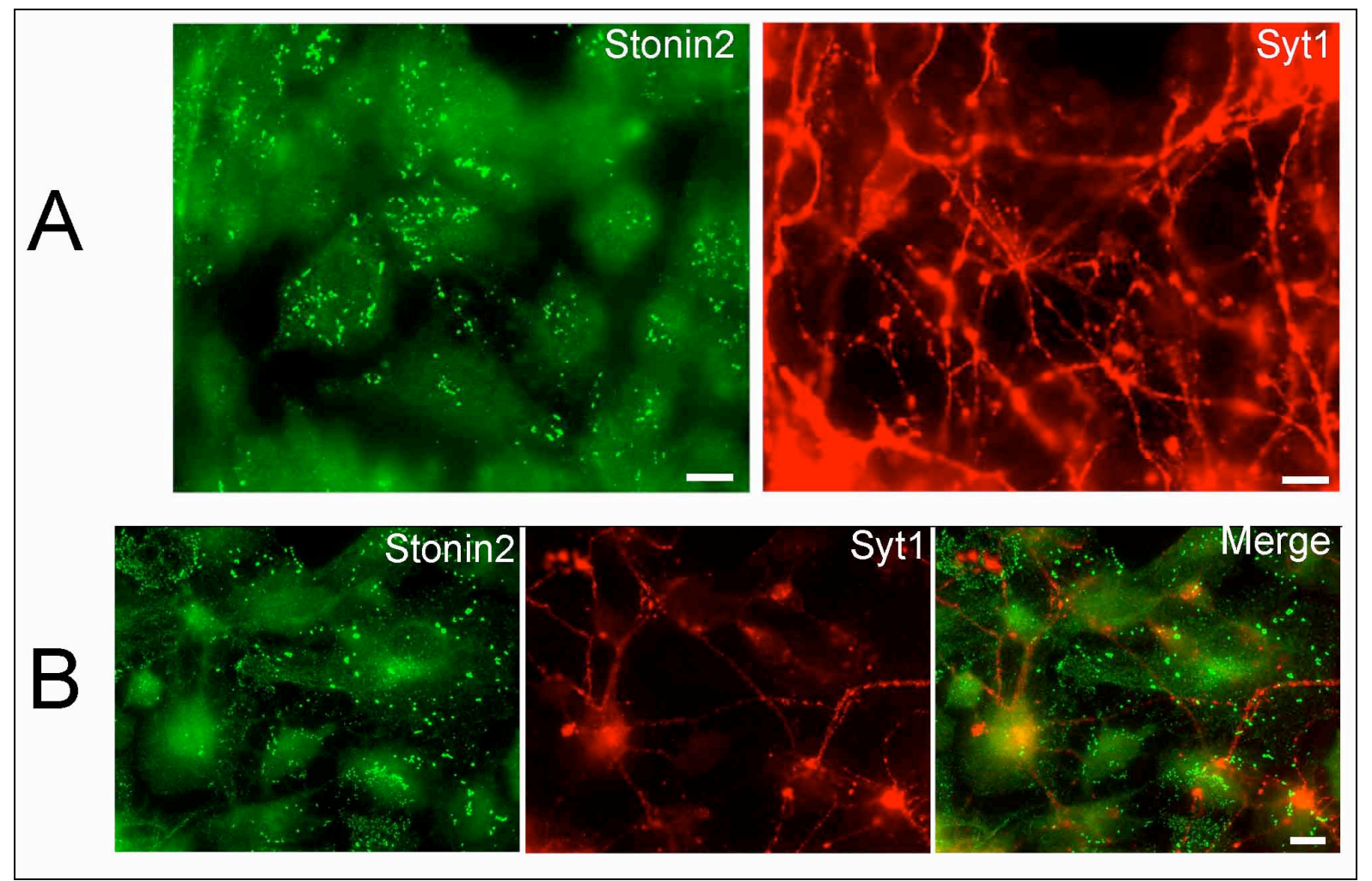

Figure 3.36 Stonin 2 immunostaining in premature neurons. (A) Embryonic stem cells were treated with retinoic acid for 10 days to induce differentiation to neuronal lineage. Expression of neuronal processes is induced in these cells together with an intense but none-punctate synaptotagmin 1 immunostaining. However, stonin 2 is expressed almost exclusively in glial cells. Scale bar, $10 \mu \mathrm{m}$. (B) Neurons obtained from day 18 mouse embryos were cultured for 4 days on coverslips to allow growth of the neuronal processes. Stonin 2 and synaptotagmin 1 do not colocalize. Scale bar, $10 \mu \mathrm{m}$.
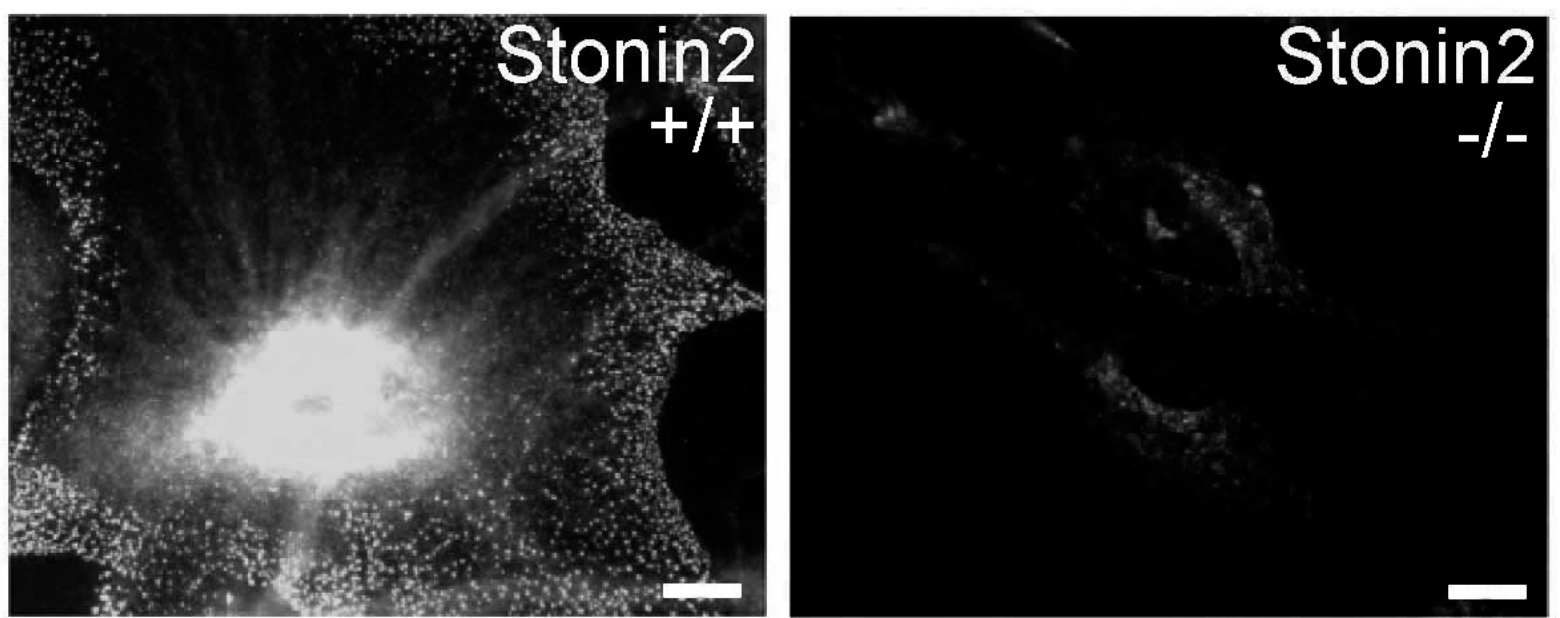

Figure 3.37 Glial immunostaining of stonin 2. Hippocampal neuron/glia mixed cultures were grown for 2 weeks in vitro and fixed and immunostained with antibodies against stonin 2. Stonin 2 has a punctate staining pattern in the underlying glial layer, which is undetectable in knock-out derived cells.

Based on the expression of glial fibrillary acidic protein (GFAP), the stonin 2immunopositive glial cells were identified as astrocytes (Figure 3.38). We also encounterd a few cells which expressed stonin 2 although no GFAP expression could 
be detected (data not shown). These could be other types of glial cells or astrocytes that do not express GFAP.
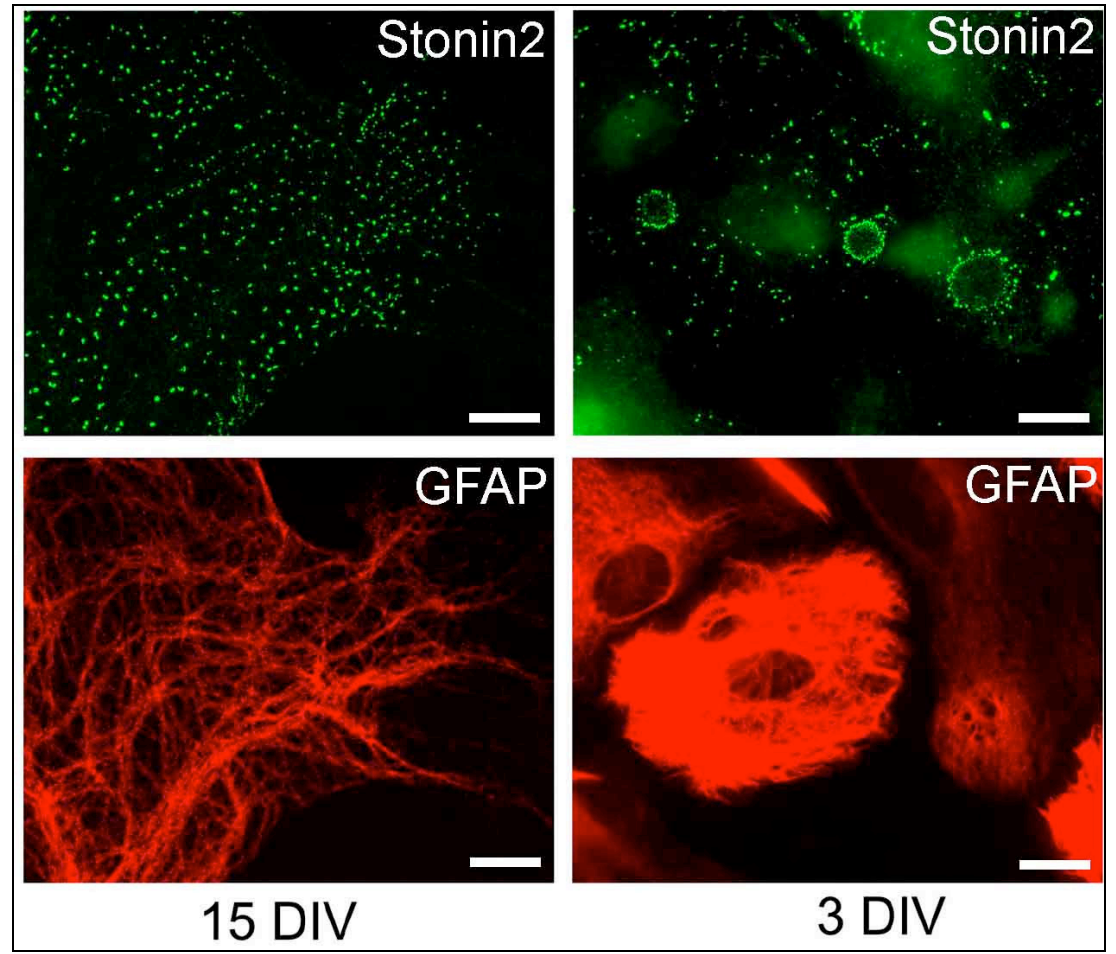

Figure 3.38 Stonin 2/GFAP double immunostaining. Glial cells grown 15 or 3 days in vitro (DIV) were double immunostained using antibodies against stonin 2 and GFAP. Stonin 2 shows a scarce punctate distribution during the early days of culturing, and stonin 2 puncta are sometimes organized into disc like structures. After culturing longer periods of time stonin 2 staining is widespread throughout the cell membrane. Scale bar, $10 \mu \mathrm{m}$.

Within these astrocytes, stonin 2 had a punctate, membrane-localized distribution reminiscent of clathrin/AP2-coated pits. Indeed, double immunostaining of astrocytes with antibodies against stonin 2 and AP2 showed a precise colocalization of the two proteins in coated-pits (Figure 3.39). It was also possible to colocalize stonin 2 with clathrin using immunofluorescence experiments, however, clathrin staining was less intense compared to that seen for AP2 staining in stonin 2 positive clusters (data not shown).

During the initial phase of culturing, stonin 2-positive clusters were few and sometimes organized into disc-like structures. Upon prolonged growth of the cells on coverslips, stonin 2 positive clusters spread throughout the cells. Close inspection of the stonin 2 staining by deconvolution microscopy revealed that, stonin 2 positive clusters were only present on the matrix-attaching side of the plasma membrane (data not shown). This suggests the possibility that stonin 2 may directly or indirectly interact extracellular matrix-associated plasma membrane factors (data not shown). 


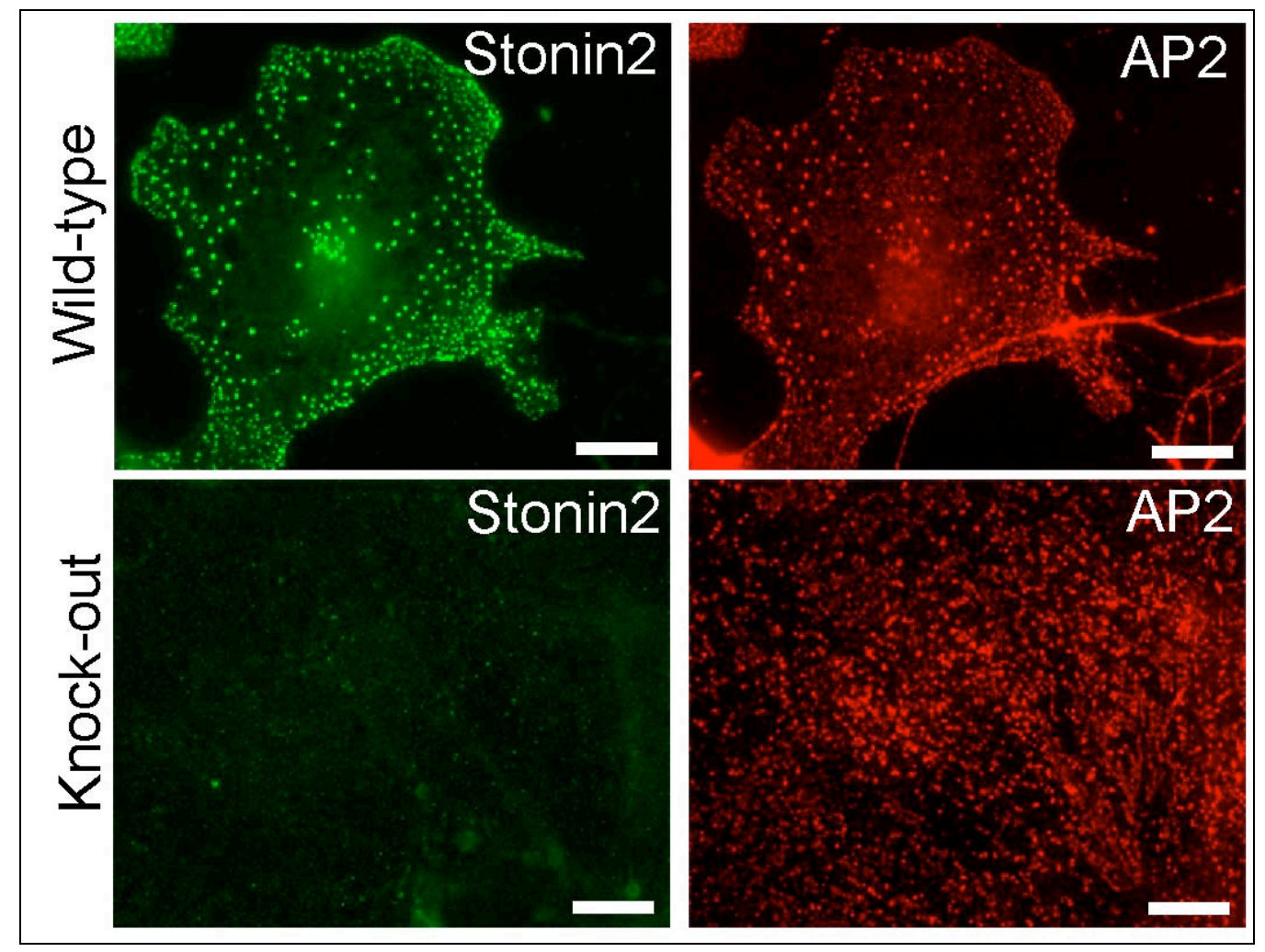

Figure 3.39 Colocalization of stonin 2 and AP2 in glial cells. Stonin 2 and AP2 colocalize at the plasma membrane large coated-pit clusters in glial cells. In the absence of stonin 2, coated pit size is smaller and there is no clustering. Scale bar, $10 \mu \mathrm{m}$.

In order to identify cell lines that endogenously expressed stonin 2, we checked glial cell lines for stonin 2 expression. U343 cell line is a human gliablastoma cell line that expresses low levels of GFAP. Western blot analysis (Figure 3.3) and immunofluorescence staining showed the presence of endogenous stonin 2 in these cells. As in astrocytes, stonin 2 was present in large clusters on the plasma membrane where it colocalized with AP2 (Figure 3.40).
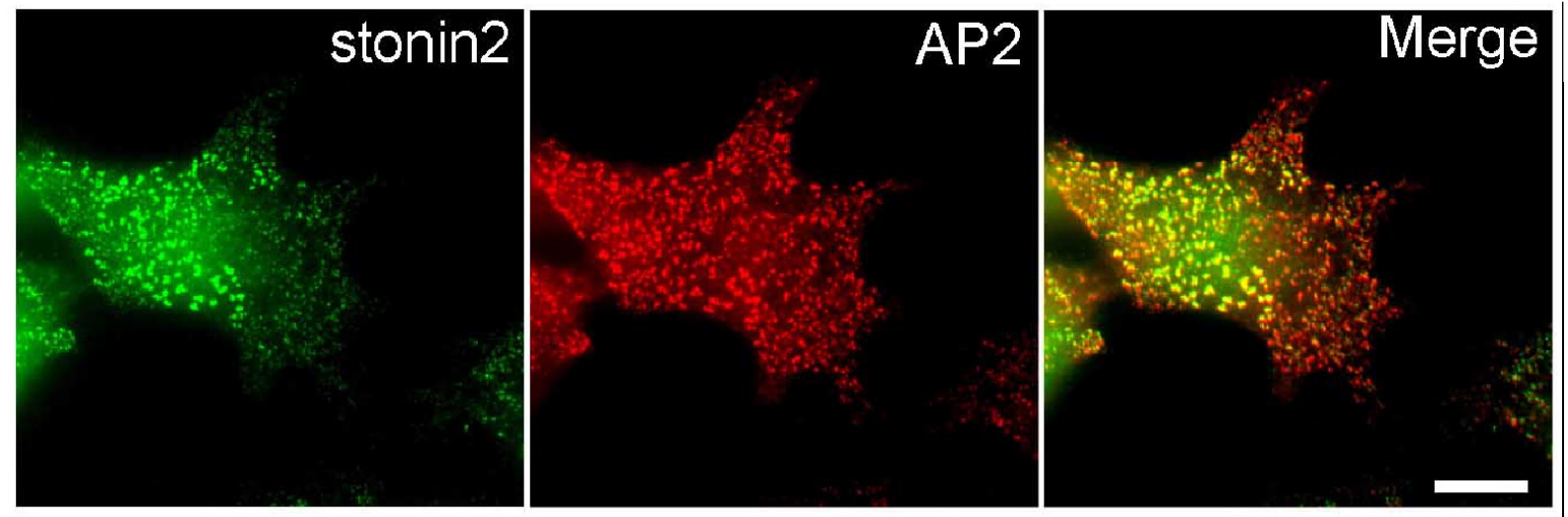

Figure 3.40 Expression and distribution pattern of stonin 2 in U343 cells. U343 cells grown on coverslips were fixed and immunostained with antibodies against stonin 2 and AP2. Both proteins colocalize at plasma membrane clusters as in astrocytes. Scale bar, $10 \mu \mathrm{m}$. 
Next, we analyzed the localization of exogenously expressed stonin 2 and stonin 1 in astrocytes. Low level transfection of astrocytes was possible using standard calciumphosphate-based transfection protocols. As seen for endogenous stonin 2, transfected EGFP-stonin 2 localized to AP2-immunopositive clusters at the plasmalemma. Surprisingly, exogenously expressed EGFP-stonin 1 showed a staining pattern identical to that seen for native stonin 2 in astrocytes (Figure 3.41). Deletion of its WVXF-type AP2 interaction motifs (data not shown), or the complete amino-terminal serine/proline-rich domain of stonin 2 (stonin $2^{\partial 420}$ ) had no effect on its localization (Figure 3.42). Therefore, localization of stonin 2 in astrocytes is independent of its $\mathrm{N}$-terminal domain and the motifs contained therein. These observations suggest that stonin 1 and stonin 2 recognize common plasma membrane factors in astrocytes.
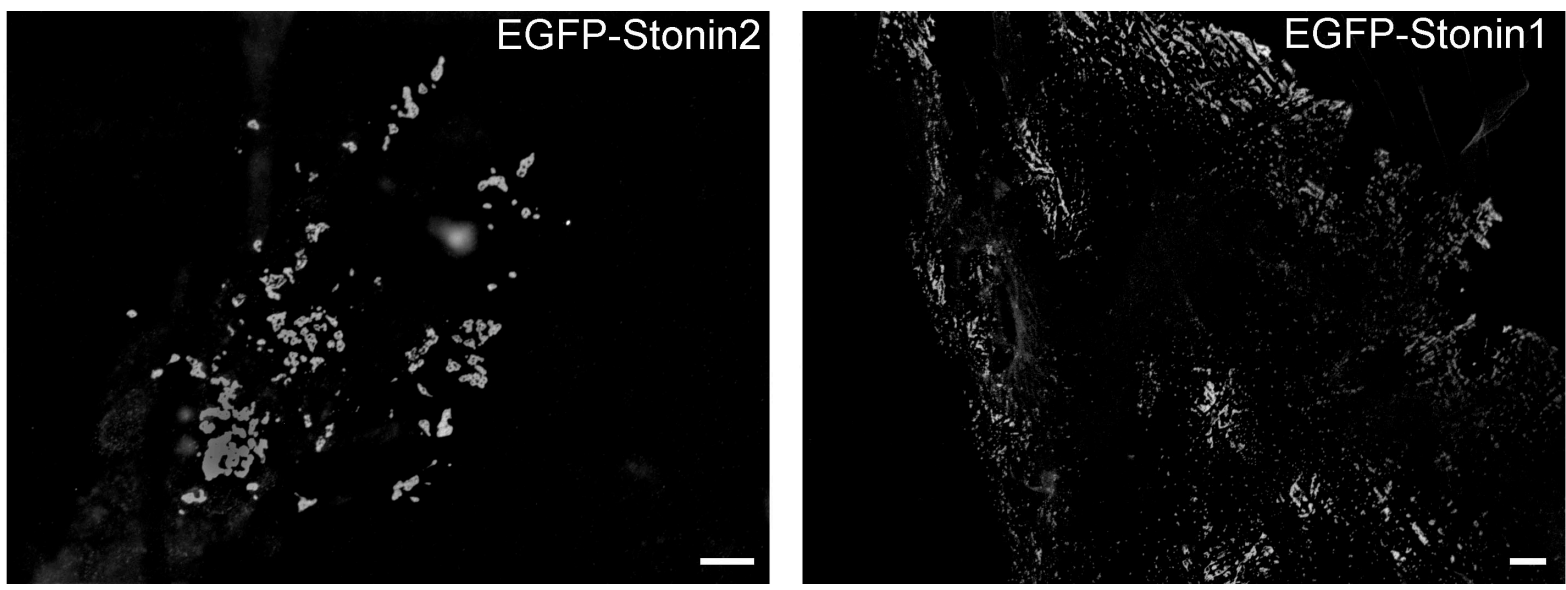

Figure 3.41 Localization of exogenously expressed EGFP-stonin 1 and stonin 2 in astrocytes. Hippocampal neuronal/glial mixed cultures were transfected with the indicated constructs using calcium-phosphate mediated DNA precipitation method. 1 week after transfection cells were fixed and EGFP fluorescence was analyzed by microscopy. Both stonin 2 and stonin 1 are present in plasma membrane cluters in astrocytes. Scale bar, $10 \mu \mathrm{m}$.
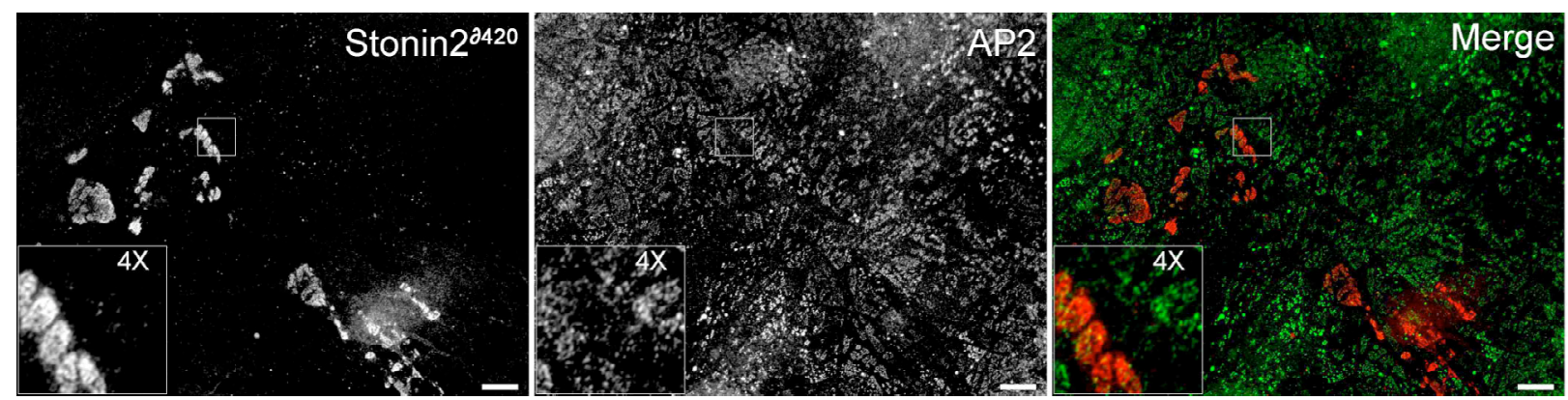

Figure 3.42 Colocalization of transfected pcHA-Stonin $2^{2420}$ with AP2 in astrocytes. Cells were transfected with pcHA-stonin $2^{\partial 420}$ construct as in figure 3.50. Double immunostaining of fixed cells with anti HA-tag and AP2 antibodies show a colocalization of both proteins in clusters. Inset shows 4 times magnification of the selected area. Scale bar, $10 \mu \mathrm{m}$. 
The presence of transfected stonin 1 in clustered coated membranes in astrocytes suggests that these cells might be expressing stonin 1 endogenously. We, therefore, decided to test whether cultured astrocytes endogenously express stonin 1 by immunofluorescence. Double immunolabeling experiments showed that stonin 1 was present in clusters of coated pits in some astrocytes although the intensity of staining was much lower than that seen for stonin 2 (Figure 3.43). This could be due to lower levels of stonin 1 in astrocytes or a lower affinity of the antibodies used for detection.

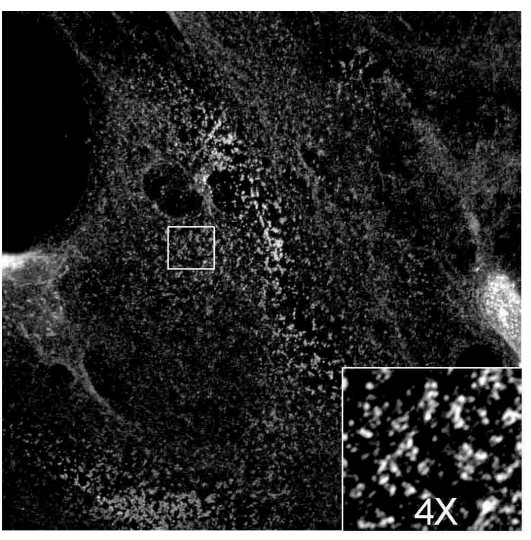

Stonin1 86.5(1:50)

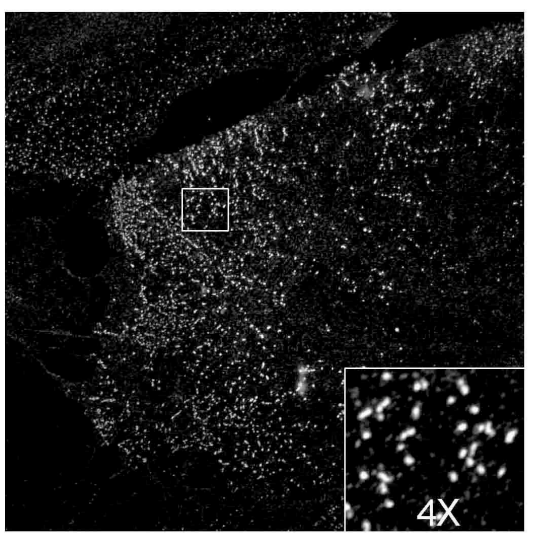

Stonin2 (1:100)

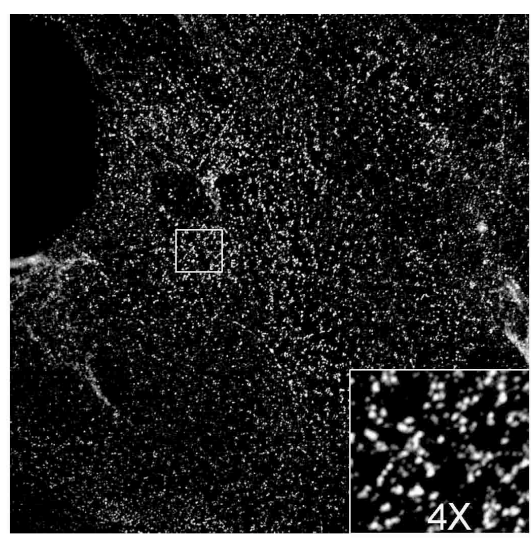

AP2

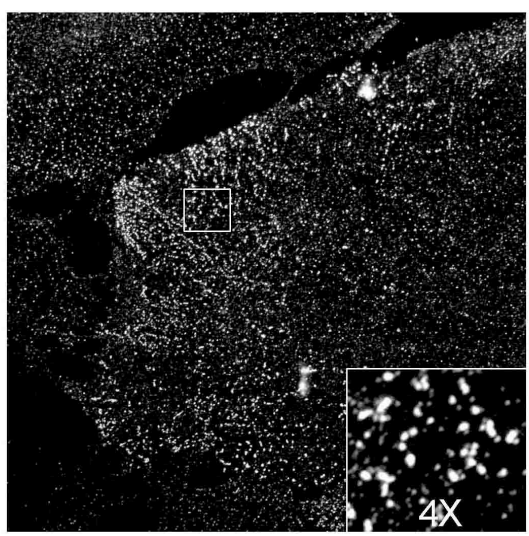

AP2

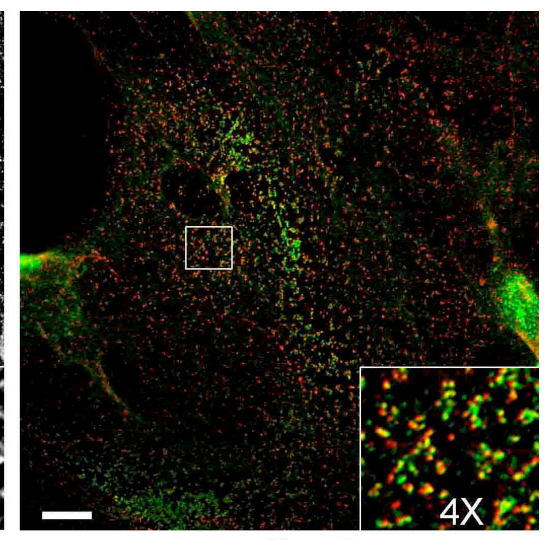

Overlay

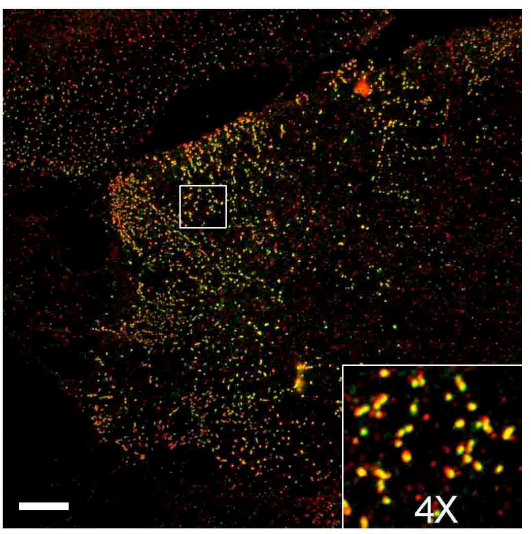

Overlay

Figure 3.43 Immunolocalization of endogenous stonin 1 and stonin 2 in astrocytes. Astrocytes cultured 2 weeks in vitro were fixed with methanol and double immunostained with the indicated antibodies. Both stonin 1 and stonin 2 colocalize with AP2 in plasma membrane coated pit clusters. Inset shows 4 times magnification of the selected area. Scale bar, $10 \mu \mathrm{m}$.

According to biochemical interaction assays, stonin 2 is able to bind to the $\mathrm{EH}$ domain containing proteins eps15 and intersectin 1 (Figure 3.5 and 3.6). If these proteins were present in astrocytes, they might interact and colocalize with stonin 2. In order to test this possibility, astrocytes were immunostained with antibodies against eps15 and intersectin 1. Indeed, both proteins were present in clusters and colocalized with both stonin 2 and AP2, respectively (Figure 3.44). Since, a stonin 2 truncation mutant that is unable to interact with these proteins still localizes to the 
same plasmalemmal structures in astrocytes, we hypothesize that stonin 2 may act by recruiting several members of the endocytic machinery to plasmalemmal enlarged coated pits.
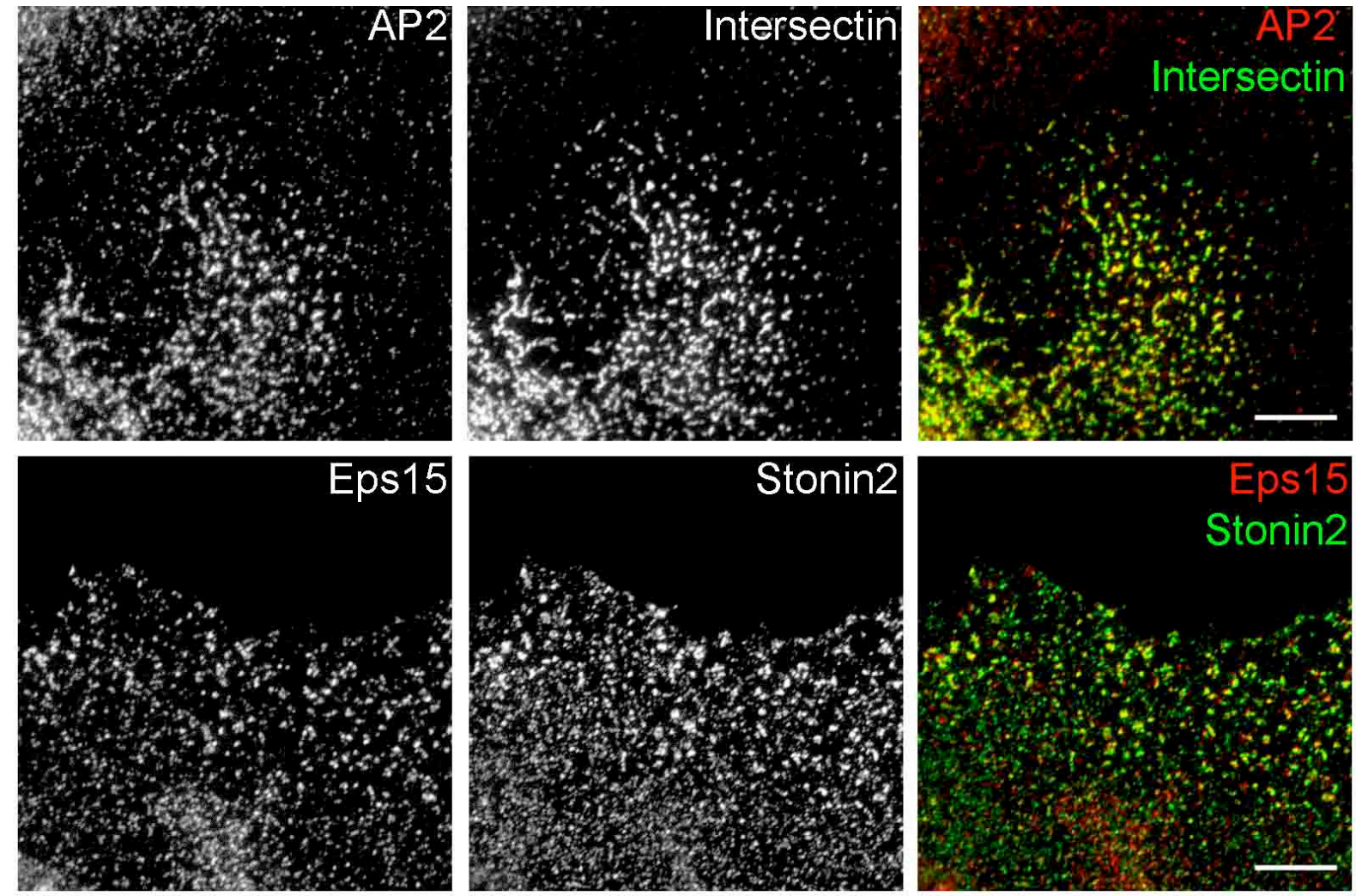

Figure 3.44 Colocalization of Eps15 and intersectin1 to plasma membrane coated pit clusters. Astrocytes were double immunostained with the indicated antibodies. Intersectin 1 colocalizes with AP2 and eps 15 with stonin 2 in the same coated pit clusters. Colocalization of intersectin 1 with stonin 2 could not be shown as both antibodies were polyclonal. Scale bar, $10 \mu \mathrm{m}$. 


\section{DISCUSSION AND CONCLUSIONS}

\subsection{Stonin 2: an alternate sorting adaptor in clathrin-mediated endocytosis}

During CME, clathrin acts as a mechanical scaffold to deform planar membranes into vesicles destined for internalization. Polymerization of clathrin triskelia at the plasmalemma is partly dependent on the presence of adaptor proteins which also bind and concentrate the cargo proteins within coated pits. AP2 is the most abundant and crucial adaptor for CME (Traub, 2003).

AP2 is able to interact with the plasma membrane by the help of two phospholipid $\left(\mathrm{PIP}_{2}\right)$ binding sites within its $\alpha$ and $\mu$ subunits, and with the clathrin terminal domain by a clathrin box motif within the hinge region connecting the $\beta$-appendage domain to the core. AP2 sorts and recruits transmembrane proteins for endocytosis by the help of two major classes of endocytic sorting motifs present within their cytosolic tails. These motifs include tyrosine-based sorting motifs with the canonical sequence $\operatorname{YXX} \Phi$ (where $X$ is any amino acid and $\Phi$ is a bulky hydrophobic residue), and dileucine motifs with the consensus sequence [DE]XXXL[LI] (Bonifacino and Traub, 2003). In addition to these, other non-canonical but structurally similar sorting motifs recognized by AP2 have also been reported (Royle et al., 2002; Wernick et al., 2005).

An important role for AP2 in clathrin-mediated endocytosis is supported by multiple lines of evidence. AP2 not only mediates the endocytic sorting of many transmembrane proteins by direct interactions but also is essential for the organization of CCPs at the plasmalemma (Nesterov et al., 1999; Motley et al.,2003; Hinrichsen et al., 2003). However, the potential diversity of membrane cargo molecules in different cell types, and the need to dynamically regulate plasma membrane targeting of many membrane receptors suggest that alternative adaptor molecules for endocytic sorting may be required.

Initial evidence suggesting the presence of additional adaptors for CME has come from studies pertaining to the endocytosis of low-density lipoprotein (LDLR) and epidermal growth factor (EGFR) receptors. Transgenic overexpression of TfR, which possesses a tyrosine-based sorting motif within its cytoplasmic tail inhibited endocytosis of endogenous TfR due to competition for the binding site within AP2- $\mu$, but did not affect LDLR and EGFR internalization (Warren et al., 1997). The EGFR 
tail harbors a canonical tyrosine motif. By contrast internalization of LDLRs requires a non-canonical FXNPXY motif which differs from YXXФ signals not only with respect to the position of the tyrosine residue but also tolerates its substitution for phenylalanine without loss of endocytic internalization (Traub, 2003).

These results suggested the presence of different intermediate sorting adaptors which direct endocytosis of LDLR and EGFR (Warrren et al., 1997).

Recent studies involving RNAi-mediated knock-down of AP2 and clathrin expression in cell lines also support this hypothesis. Although AP2 deficiency inhibits endocytic internalization of TfR, it has limited effects on LDLR and EGFR uptake. By contrast, clathrin knock-down eliminates internalization of all three receptors (Motley et al., 2003; Hinrichsen et al., 2003).

In combination, these and other studies (Conner and Schmid, 2003), suggest that alternate cargo adaptors distinct from AP2 are actively involved in CME of select cargo proteins.

The characteristic features usually shared by alternate sorting adaptors include: a structurally unfolded domain harboring motifs recognized by AP2 or clathrin (motif domains, Praefcke et al., 2004), a cargo binding domain that recognizes distinct cargo molecules or sorting motifs therein, and a phospholipid binding domain that aids in the recruitment of these molecules to the plasma membrane (Traub, 2003).

The alternate sorting adaptor molecules include i) $\beta$-arrestins (endocytosis of $G$ protein-coupled plasma membrane receptors, GPCR), ii) ARH, Disabled-2 and numb (endocytic sorting of LDLR types by the recognition of FXNPXY motifs via their phosphotyrosine binding domain), and iii) epsin 1, AP180/CALM, HIP1/ HIP1R (via presence of $\mathrm{PIP}_{2}$-binding ENTH/ANTH domains and ubiquitin interacting motifs in the case of epsins) (see Table 4.1).

We believe that stonin 2 is a member of the growing family of endocytic adaptor molecules with a predominant function in clathrin-mediated endocytosis of synaptotagmins. This hypothesis is supported by the following lines of evidence:

i) direct interaction with the endocytic machinery via NPF and AP2-binding WVXF motifs; 
ii) the ability to specifically recognize synaptotagmins as putative membrane cargo;

iii) the ability to facilitate internalization of synaptotagmins via an AP2dependent internalization mechnism and

iv) the ability to regulate sorting synaptotagmins to neurosecretory vesicles (i.e. SLMVs in PC12 cells).

\begin{tabular}{|c|c|c|c|c|}
\hline $\begin{array}{l}\text { Adaptor } \\
\text { molecule }\end{array}$ & Features & $\begin{array}{l}\text { Cargo } \\
\text { binding } \\
\text { modules }\end{array}$ & $\begin{array}{l}\text { Cargo } \\
\text { recognized }\end{array}$ & $\begin{array}{l}\text { Related } \\
\text { adaptor } \\
\text { molecules }\end{array}$ \\
\hline ARH1 & $\begin{array}{l}\text { PTB domain binds to plasma } \\
\text { membrane } \mathrm{PIP}_{2} \text { and sorting motifs } \\
\text { High affinity binding to } \beta \text {-appendage } \\
\text { domain, clathrin box in carboxy- } \\
\text { terminal domain }\end{array}$ & $\begin{array}{l}\text { amino } \\
\text { terminal } \\
\text { phospho } \\
\text { tyrosine } \\
\text { binding } \\
\text { domain } \\
\text { (PTB) }\end{array}$ & $\begin{array}{l}\text { FXNPXY motifs } \\
\text { within the } \\
\text { cytosolic tails of } \\
\text { LDL receptors }\end{array}$ & $\begin{array}{l}\text { Disabled-2 } \\
\text { Numb }\end{array}$ \\
\hline $\begin{array}{l}\beta- \\
\text { arrestins }\end{array}$ & $\begin{array}{l}\mathrm{PIP}_{2} \text {-binding sites } \\
\text { Carboxy-terminal domain contains } \\
\text { clathrin and } \alpha \text {-appendage binding } \\
\text { sites which become available only } \\
\text { upon bindníng to cargo receptors }\end{array}$ & $\begin{array}{l}\text { amino- } \\
\text { terminal part } \\
\text { of the protein }\end{array}$ & $\begin{array}{l}\text { phosphorylated } \\
\text { G-protein coupled } \\
\text { plasma } \\
\text { membrane } \\
\text { receptors (GPCR) }\end{array}$ & $\begin{array}{l}\beta \text {-arrestins } \\
1 \text { and } 2\end{array}$ \\
\hline Epsin1 & $\begin{array}{l}\text { Amino-terminal } \mathrm{PIP}_{2} \text { binding ENTH } \\
\text { domain (epsin } \mathrm{N} \text {-terminal homology), } \\
\text { NPF motifs that bind to EH domains } \\
\text { Contains clathrin and } \alpha \text {-appendage } \\
\text { binding motifs }\end{array}$ & $\begin{array}{l}\text { UIMs } \\
\text { (ubiquitin- } \\
\text { interacting } \\
\text { motifs) }\end{array}$ & $\begin{array}{l}\text { Mono- } \\
\text { ubiquitinated } \\
\text { proteins }\end{array}$ & $\begin{array}{l}\text { EpsinR } \\
\text { AP180 } \\
\text { /CALM } \\
\text { HIP1 } \\
\text { HIP1R }\end{array}$ \\
\hline Stonin 2 & $\begin{array}{l}\text { Amino terminal AP2-binding motifs, } \\
\text { NPF motifs }\end{array}$ & $\begin{array}{l}\mu \text {-homology } \\
\text { domain }\end{array}$ & $\begin{array}{l}\text { Synaptotagmins } \\
1,2 \text { and } 9(8 ?)\end{array}$ & Stonin 1 \\
\hline
\end{tabular}

Table 4.1 Alternate sorting adaptors in CME. Alternate sorting adaptors in CME have been categorized according to the protein domains they contain. Note that, the role of epsins as well as eps15 as adaptor molecules in CME of ubiquitinated cargo is uncertain as recent studies suggest a role in clathrin-independent endocytosis (Sigismund et al., 2005; Chen and De Camilli, 2005).

WVXF-type binding motifs define a novel class of accessory protein binding signals in CME. Quite recently, the molecular structure of the $\alpha$-adaptin appendage domain in complex with WVXF-type peptides has been solved (Praefcke et al., 2004; Ritter et al., 2004; Mishra et al., 2004). WVXF-type peptides from stonin 2 or synaptojanin 1 bind to a site within the $\alpha$-appendage domain (sandwich subdomain) that is different from the site (platform subdomain) used for interaction with DPF/W and FXDXF type motifs (Brett et al., 2002; see Figure 3.16). DPW motifs were shown to have a lower affinity interaction with the stonin 2 binding site on the sandwich subdomain. 
However, the peptide sequences are recognized in opposite orientations in the two cases (Praefcke et al., 2004). The WVXF-type peptides interact with an extended surface on the $\alpha$-sandwich subdomain. The tryptophane and phenylalanine residues are recognized by shallow hydrophobic pockets created by side chains of the $\beta$-sheet residues. Mutations of the residues that form these pockets (i.e. F740D, G742D, G725N or E) abolished the interactions with WVXF peptides but had no effect on binding to eps15 via DPF/W. By contrast, mutations of the residues required for binding to DPF/W or FXDXF type motifs (i.e. W840A) had no effect on binding to WVXF-harboring proteins but abolished binding to eps15 (Praefcke et al., 2004; Mishra et al., 2004). In addition to bulky aromatic side-chains, the negatively charged residues also play a major role in recognition of WVXF motifs. An electrostatic potential analysis of the sandwich subdomain revealed a cluster of positively charged residues ( $K, R$ and $H$ ), that would lie in a complementary location to the acidic residues that flank the WVXF-motif on its carboxy-terminal side (Ritter et al., 2004). The dissociation constants for the binding of synaptojanin 1 WVXF peptides to the $\alpha$ appendage domain have been measured by isothermal calorimetry and found to be as low as $0.7 \pm 0.1 \mu \mathrm{M}$. This is one of the highest affinity interactions reported between peptide motifs and the AP2- $\alpha$-adaptin appendage domain (Praefcke et al., 2004).

With the identification of these novel binding sites for WVXF-type sorting motifs within the sandwich subdomain of the $\alpha$-appendage (Walther e al., 2004; Ritter et al., 2003; Jha et al., 2004), and the recent analysis of the interaction surface for ARH1 and $\beta$ arrestins within the $\beta$-appendage domain (Mishra et al., 2005), the number of accessory factor interaction sites in the AP2 complex has increased to three. What might be the physiological significance for having distinct binding sites for different proteins in endocytosis and sorting? One explanation for this observation might be that, in order to avoid extensive competition for AP2 binding, these proteins might have evolved alternative mechanisms of AP2 association. The employment of a relatively rarely used binding site for association with clathrin-coated pits would ensure efficient sorting of select cargo molecules by alternate adaptors. A privileged site would prevent alternate adaptor molecules including stonin 2 from being displaced by other abundant accessory proteins like epsin1 or AP180/CALM. The presence of an alternative AP2 binding site could also have implications during the assembly of an endocytic protein network underneath the clathrin coat. For instance, 
independent interactions between AP2, eps15 and stonin 2 would enhance the stability of macromolecular endocytic protein complexes.

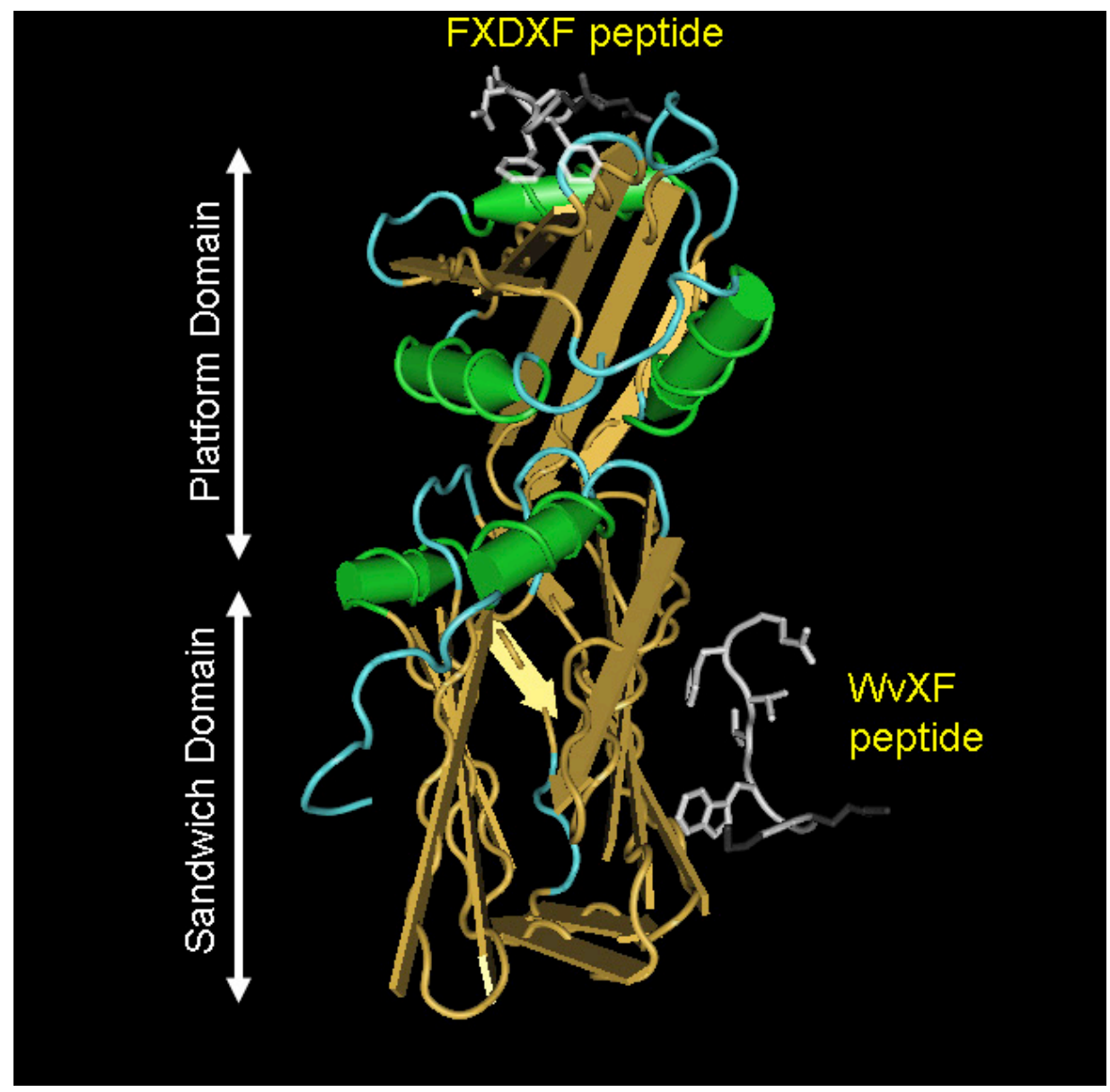

Figure 4.1 Crystal structure of $\alpha$-adaptin appendage domain in complex with WVXF and FXDXF type peptides. NPKGWVTFEEEE and LDGFKDSFDLQG peptides from the C-terminus of synaptojanin 1 long-splice isoform bound to $\alpha$-adaptin appendage domain were crystallized and the structure was solved. Secondary structure elements are shown in a color code: $\alpha$-helices (green rods), $\beta$-sheets (golden plates) and unfolded loops (light blue). Peptides were shown as gray tubes. The structure file was retrieved from Protein Data Bank (structure ID 1W80; Praefcke et al., 2004) and rendered by Cn3D software for preparation of the figure.

\subsection{Stonin 2 in synaptotagmin 1 endocytosis and sorting}

Coupled exo- and endocytosis of vesicles proceeds at high rates in active nerve terminals. Action potentials arrive at the presynaptic compartment at frequencies as high as $50 \mathrm{~Hz}$ causing fusion of SVs with the underlying plasma membrane. If synapses were unable to regenerate and refill their SVs quickly, they would loose the ability to secrete neurotransmitters within tens of seconds. Synaptotagmin 1 is a SV protein with vital roles in both exocytosis and endocytosis (Schwarz, 2004). 
There is extensive genetic, biochemical and electrophysiological evidence linking the synaptic vesicle protein synaptotagmin 1 to $\mathrm{Ca}^{2+}$-stimulated SV exocytosis (Sudhof, 2004). By contrast, the role of synaptotagmin 1 in compensatory vesicle endocytosis following SV fusion has been partially neglected due to the lack of experimental methods for analysis. Recent studies, using synaptotagmin 1 deficient mouse and fly models, confirm its crucial role in SV endocytosis (Poskanzer et al., 2003; NicholsonTomishima and Ryan, 2004).

In an elegant study by Poskanzer et al., transgenically expressed synaptopHlourin (Miesenbock et al., 1998) was exploited to assay endocytosis in Drosophila NMJs after inactivation of synaptotagmin 1. A novel technique enabled precisely timed inactivation of synaptotagmin 1 just prior to endocytosis to avoid secondary effects of disrupted exocytosis. This was achieved by reversible blocking of the vesicle fission event in Drosophila shibire mutants and fluorescence-light assisted inactivation (FALI) of tagged synaptotagmin 1. The results indicate that synaptotagmin 1 is required for endocytosis of $\mathrm{SVs}$ that had undergone $\mathrm{Ca}^{2+}$ exocytosis using functional synaptotagmin 1 prior to FALI-mediated inactivation (Poskanzer et al., 2003).

A number of previous studies have indicated a role for synaptotagmin 1 in clathrin/AP2-mediated endocytosis of SVs (Zhang et al., 1994; Haucke and De Camilli 1999; Haucke et al., 2000). Synaptotagmin 1 is recognized by AP2- $\mu$ via a polybasic stretch of residues within its C2B domain (Haucke et al., 2000; Grass et al., 2004). In addition, the WHXL motif in its carboxy-terminal part and $\mathrm{N}$ - and $\mathrm{O}$ glycosylation of its lumenal tail have also been proposed to regulate endocytosis and proper targeting of synaptotagmin 1 (Jarousse et al., 2003; Han et al., 2004; AtiyaNasagi et al., 2005).

Genetic, biochemical and physiological evidence have shown that Drosophila stoned B protein is essential for endocytic recycling of SVs. However, the synaptic defects associated with a mutant stoned locus seem to be exclusively caused by a partial loss of function of synaptotagmin 1. Stoned mutations cause mislocalization and degradation of synaptotagmin 1 and overexpression of synaptotagmin 1 in stoned flies is sufficient to correct the physiological abnormalities seen in stoned nerve terminals (Fergestad and Broadie, 2001).

The interaction of AP2 with synaptotagmin 1 in the context of CME is well established (Takei and Haucke, 2001). Then what might be the underlying reasons to make use 
of an additional adaptor molecule like stoned B/stonin 2 for endocytosis of synaptotagmin 1? Although we cannot give a conclusive answer to this question yet, several lines of evidence suggest that stoned B/stonin 2 selectively facilitates internalization and perhaps endosomal sorting of synaptotagmin family members.

Our findings pertaining to stonin 2-mediated synaptotagmin internalization parallel those made recently for ligand-activated receptor tyrosine kinases such as the EGFR. In the presence of low concentrations of extracellular EGF, the EGFR is almost exclusively internalized through CME probably by direct interaction of its sorting motif with the AP2 complex. However, higher concentrations of EGF lead to ubiquitination of the receptor tails and internalization by a clathrin-independent, caveolin/raft dependent pathway. Eps15 acts as an adaptor molecule by recognizing the ubiquitinated EGFR via its ubiquitin interaction motifs. EGFR internalized by the second pathway is thought to be targeted for degradation. Thus, the presence of an alternate adaptor molecule (i.e. eps15 in this case), is essential for information processing by these cells.

Although, unlike eps15, stonin 2 requires the presence of AP2 for synaptotagmin 1 internalization, it may act by preferentially facilitating incorporation of synaptotagmin 1 but not other receptors into newly forming clathrin-coated vesicles within presynaptic nerve terminals.

Ectopically expressed synaptotagmin 1 gets stranded at the plasmalemma in fibroblasts (Feany et al., 1993a and b), although these cells contain normal amounts of AP2 and a functional CME machinery, they were found to be incompetent for endocytic internalization of synaptotagmin 1 . We have shown here that, stonin 2 can facilitate synaptotagmin 1 internalization as much as 20-fold in HEK293 or PC12 cells by physically linking it to the AP2 complex and the endocytic machinery.

Second, our studies in neurosecretory PC12 cells show that, the functions of stonin 2 may not be restricted to the internalization of synaptotagmin 1. Overexpression of synaptotagmin 1 in these cells, as in fibroblasts, leads to the partial accumulation of synaptotagmin 1 at the plasma membrane. Although these cells endogenously express synaptotagmin 1 which is internalized by CME (Jarousse and Kelly, 2001a), overexpression of stonin 2 results in a more than 10-fold stimulation of synaptotagmin 1 internalization. In the absence of exogenously expressed stonin 2 , internalized synaptotagmin 1 is targeted to uncharacterized presumably endosomal 
compartments distinct from SLMVs. In addition to facilitating its internalization, stonin 2 also assists in correct sorting of synaptotagmin 1 to SLMVs. Therefore stonin 2 may serve a dual function in internalization and SLMV-targeting of synaptotagmin 1. In support of this hypothesis, we find stonin 2 colocalizing with synaptotagmin 1 in endosomes in transfected HEK293 cells.

\subsection{Stonin 2 expression in astrocytes}

During the course of this study, we discovered that stonin 2 expression is not constrained to neurons and neurosecretory cells. Astroglial cells also display high level expression of stonin 2 which localizes to coated-pits at the plasma membrane, where it colocalizes precisely with AP2. The size of AP2/stonin 2-containing coatedpits is sometimes abnormally large reaching several micrometers in diameter. Stonin 2 seems to be an essential factor for the morphological organization of such 'clusters of coated pits', since these structures are absent from knock-out astrocytes. Interaction of stonin 2 with putative cargo protein(s) at the plasma membrane of astrocytes might be directed by its $\mu \mathrm{HD}$ since deletion of its amino-terminal domain does not have any effect on recruitment to coated-pits.

Recent studies in astroglial cells have demonstrated the presence of a secretory machinery similar to the SV secretory machinery utilized for stimulated exocytosis in neurons. Release of messenger molecules (i.e. glutamate) from astrocytes might modulate neurotransmission or constitute part of a glial transmission system (Wilhelm et al., 2004; Matthias et al., 2003). None of the stonin 2-interacting synaptotagmins (1,2 and 9) are known to be expressed in astrocytes. Synaptotagmin 4, which is expressed in astrocytes and regulates $\mathrm{Ca}^{2+}$-stimulated glutamate release (Zhang et al., 2004), does not interact with stonin 2 in our in vivo interaction assays based on colocalization. We therefore believe that stonin 2 as well as stonin 1 may have additional cargo molecules in astrocytes. It will thus be interesting to analyze whether glial secretion or glutamate receptor expression are influenced in stonin 2 knock-out animals.

\subsection{Analysis of stonin 2 knock-out mice}

Within the course of this project, we generated a stonin 2 deficient mouse strain by targeted deletion of the stonin 2 gene. Our expectations were to reproduce the phenotypic characteristics of Drosophila stoned B in mice. Unexpectedly, stonin 2 
animals were viable and our preliminary analyses yielded no abnormalities with regard to synaptotagmin 1 distribution or synaptic vesicle recycling.

Synaptotagmin 1 distribution was mainly analyzed by biochemical techniques. Unlike findings made in stoned mutant flies we did not observe a decrease in synaptotagmin 1 expression or its mislocalization to the presynaptic plasma membrane. Immunofluorescence microscopy showed that the synaptic morphology and density of synapses was similar in cultured neurons isolated from WT or KO animals. Preliminary analysis of synaptic vesicle recycling (performed by Dr. J. Klingauf's group, MPI Biophysical Chemistry, Göttingen) by electrophysiological techniques involving FM dyes or transfection of a synaptopHlourin construct did not reveal any significant differences between KO and WT animals (data not shown). The initial analysis of stonin 2 deficient nerve terminals using electron microscopy (performed by Prof. G. Ahnert-Hilger's group, Charite, Berlin) also did not yield any alterations with regard to synaptic vesicle morphology, number or distribution in the presynaptic compartment.

All of these data suggest that the absence of stonin 2 may be compensated by other factors during synaptic vesicle recycling. One likely candidate gene that could be substituting stonin 2 is stonin 1 . However, the experimental data presented in this thesis suggest that this is unlikely. Stonin 1 does not interact with synaptotagmin in vitro or in vivo. In our antibody uptake assays, expression of stonin 1 did not have any effects on synaptotagmin 1 internalization. In addition, in transfected neurons, (unlike stonin 2) stonin 1 does not localize to presynaptic vesicle clusters but remains soluble. The identity of the factor or factors that might compensate for stonin 2 deficiency in synaptotagmin 1 endocytosis remain elusive at this point.

Our future experiments will focus on the better analysis of stonin 2 knock-out mice and identification of cargo molecules other than synaptotagmins endocytosed and sorted by stonin 1 and stonin 2 . The former will be pursued by a thorough electrophysiological analysis of stonin 2 deficient nerve terminals and EM analysis of the presynaptic synaptotagmin 1 distribution via immunogold labelling. The latter will require affinity purification of putative cargo molecule(s) using recombinant stonin 2 proteins. A proteomics approach involving purification of CCVs from WT or KO astrocytes is also being considered. 


\subsection{Summary and Conclusions}

The major findings and novel discoveries of this study can be summarized as follows;

- We confirmed our previous results (Walther et al., 2001) with regard to brainspecific expression of stonin 2. We discovered stonin 2 expression in secretory cell types like chromaffin and pancreatic islet cells. Furthermore, stonin 2 was localized to synaptic vesicle clusters in cultured neurons and to plasma membrane coated-pits in astroglial cells.

- A novel AP2- $\alpha$-appendage binding motif (WVXF-motif) was discovered within stonin 1 and stonin 2 as well as other endocytic accessory proteins.

- Stonin 2 was shown to interact with synaptotagmin 1 in vivo by coimmunoprecipitation assays and in N1E cells and neurons by colocalization and membrane recruitment experiments. Stonin $2-\mu \mathrm{HD}$ was found to be required for synaptotagmin 1 interaction. Other synaptotagmins ( 2 and 9 ) were also shown to recruit stonin 2 to the membranes. Stonin 1 was found not to interact with any of the synaptotagmins assayed.

- Coexpression of stonin 2 and synaptotagmin 1 in N1E cells resulted in translocation of otherwise cytosolic stonin 2 to plasma membrane clathrin/AP2-coated pits.

- Coexpression of stonin 2 but not stonin 1 was shown to facilitate endocytosis of plasma membrane-stranded synaptotgmin 1 in fibroblasts as well as in neurosecretory PC12 cells.

- Stonin 2-assisted-internalization of synaptotagmin 1 depended on the presence of a functional $\mu \mathrm{HD}$ and its ability to be recruited to coated-pits via association with the AP2 complex. Mutagenesis of WVXF-type motifs significantly reduced synaptotagmin 1 uptake. The importance of AP2 in synaptotagmin 1 endocytosis was further confirmed by RNAi-mediated gene knock-down experiments. Stonin 2 was unable to assist synaptotagmin 1 uptake in an AP2 deficient background.

- Experiments in neuroendocrine PC12 cells showed that stonin 2 may also aid proper targeting of endocytosed synaptotagmin 1 to synaptic-like microvesicles. 
- In order to obtain further information about stonin 2 functions in endocytic recycling SVs, a stonin 2-deficient mouse strain was generated by targeted gene inactivation. Stonin 2 knock-out mice were found to be viable and fertile. Preliminary analysis of stonin 2 knock-out mice with respect to synaptotagmin 1 distribution, SV recycling, morphology and distribution yielded no abnormalities compared to WT littermates.

- Stonin 2 as well as stonin 1 were found to localize to large clusters of coatedpits at plasma membranes of astrocytes. Association of stonin 2 with the plasma membrane was independent of its binding to endocytic proteins like AP2, intersetin 1 or eps 15 but presumably mediated by the interactions of its $\mu \mathrm{HD}$ with unidentified receptor(s) at the plasmalemma.

We thus propose that stonin 2 is an endocytic adaptor molecule with functions in endocytosis and targeting of synaptotagmins 1,2 and 9 as well as yet unidentified plasma membrane receptors in astroglial cells. 


\section{REFERENCES}

Aguilar, R.C. and Wendland, B. (2005) Endocytosis of membrane receptors: two pathways are better than one. Proc Natl Acad Sci U S A, 102, 2679-2680.

Alberts, B., Johnson, A., Lewis, J., Raff, M., Roberts, K. and Walter, P. (2002) Molecular Biology of the Cell. Garland Publishing, New York.

Atiya-Nasagi, Y., Cohen, H., Medalia, O., Fukudan, M. and Sagi-Eisenberg, R. (2005) Oglycosylation is essential for intracellular targeting of synaptotagmins I and II in nonneuronal specialized secretory cells. J Cell Sci, 118, 1363-1372.

Banker, G. and Goslin, K. (1998) Culturing Nerve Cells. MIT Press, Massachusetts.

Blondeau, F., Ritter, B., Allaire, P.D., Wasiak, S., Girard, M., Hussain, N.K., Angers, A., Legendre-Guillemin, V., Roy, L., Boismenu, D., Kearney, R.E., Bell, A.W., Bergeron, J.J. and McPherson, P.S. (2004) Tandem MS analysis of brain clathrin-coated vesicles reveals their critical involvement in synaptic vesicle recycling. Proc Natl Acad Sci U S A, 101, 3833-3838.

Bockamp, E., Maringer, M., Spangenberg, C., Fees, S., Fraser, S., Eshkind, L., Oesch, F. and Zabel, B. (2002) Of mice and models: improved animal models for biomedical research. Physiol Genomics, 11, 115-132.

Boehm, M. and Bonifacino, J.S. (2001) Adaptins: the final recount. Mol Biol Cell, 12, 29072920.

Bonifacino, J.S. and Traub, L.M. (2003) Signals for sorting of transmembrane proteins to endosomes and lysosomes. Annu Rev Biochem, 72, 395-447.

Brett, T.J., Traub, L.M. and Fremont, D.H. (2002) Accessory protein recruitment motifs in clathrin-mediated endocytosis. Structure (Camb), 10, 797-809.

Brodsky, F.M., Chen, C.Y., Knuehl, C., Towler, M.C. and Wakeham, D.E. (2001) Biological basket weaving: formation and function of clathrin-coated vesicles. Annu Rev Cell Dev Biol, 17, 517-568.

Chapman, E.R. (2002) Synaptotagmin: a Ca(2+) sensor that triggers exocytosis? Nat Rev Mol Cell Biol, 3, 498-508.

Chen, H. and De Camilli, P. (2005) The association of epsin with ubiquitinated cargo along the endocytic pathway is negatively regulated by its interaction with clathrin. Proc Natl Acad Sci U S A, 102, 2766-2771.

Collins, B.M., McCoy, A.J., Kent, H.M., Evans, P.R. and Owen, D.J. (2002) Molecular architecture and functional model of the endocytic AP2 complex. Cell, 109, 523-535.

Confalonieri, S. and Di Fiore, P.P. (2002) The Eps15 homology (EH) domain. FEBS Lett, $513,24-29$.

Conner, S.D. and Schmid, S.L. (2002) Identification of an adaptor-associated kinase, AAK1, as a regulator of clathrin-mediated endocytosis. J Cell Biol, 156, 921-929.

Conner, S.D. and Schmid, S.L. (2003) Regulated portals of entry into the cell. Nature, $\mathbf{4 2 2}$, 37-44. 
Conner, S.D., Schroter, T. and Schmid, S.L. (2003) AAK1-mediated micro2 phosphorylation is stimulated by assembled clathrin. Traffic, 4, 885-890.

Corpet, F. (1988) Multiple sequence alignment with hierarchical clustering. Nucleic Acids Res, 16, 10881-10890.

Cremona, O. and De Camilli, P. (1997) Synaptic vesicle endocytosis. Curr Opin Neurobiol, 7, 323-330.

Cremona, O., Di Paolo, G., Wenk, M.R., Luthi, A., Kim, W.T., Takei, K., Daniell, L., Nemoto, Y., Shears, S.B., Flavell, R.A., McCormick, D.A. and De Camilli, P. (1999) Essential role of phosphoinositide metabolism in synaptic vesicle recycling. Cell, 99, 179-188.

Dafforn, T.R. and Smith, C.J. (2004) Natively unfolded domains in endocytosis: hooks, lines and linkers. EMBO Rep, 5, 1046-1052.

Deak, F., Schoch, S., Liu, X., Sudhof, T.C. and Kavalali, E.T. (2004) Synaptobrevin is essential for fast synaptic-vesicle endocytosis. Nat Cell Biol, 6, 1102-1108.

Di Paolo, G., Moskowitz, H.S., Gipson, K., Wenk, M.R., Voronov, S., Obayashi, M., Flavell, R., Fitzsimonds, R.M., Ryan, T.A. and De Camilli, P. (2004) Impaired Ptdlns(4,5)P2 synthesis in nerve terminals produces defects in synaptic vesicle trafficking. Nature, 431, 415-422.

Di Paolo, G., Sankaranarayanan, S., Wenk, M.R., Daniell, L., Perucco, E., Caldarone, B.J., Flavell, R., Picciotto, M.R., Ryan, T.A., Cremona, O. and De Camilli, P. (2002)

Decreased synaptic vesicle recycling efficiency and cognitive deficits in amphiphysin 1 knockout mice. Neuron, 33, 789-804.

Drab, M., Verkade, P., Elger, M., Kasper, M., Lohn, M., Lauterbach, B., Menne, J., Lindschau, C., Mende, F., Luft, F.C., Schedl, A., Haller, H. and Kurzchalia, T.V. (2001) Loss of caveolae, vascular dysfunction, and pulmonary defects in caveolin-1 gene-disrupted mice. Science, 293, 2449-2452.

Elbashir, S.M., Harborth, J., Lendeckel, W., Yalcin, A., Weber, K. and Tuschl, T. (2001) Duplexes of 21-nucleotide RNAs mediate RNA interference in cultured mammalian cells. Nature, 411, 494-498.

Estes, P.S., Jackson, T.C., Stimson, D.T., Sanyal, S., Kelly, L.E. and Ramaswami, M. (2003) Functional dissection of a eukaryotic dicistronic gene: transgenic stonedB, but not stonedA, restores normal synaptic properties to Drosophila stoned mutants. Genetics, $165,185-196$.

Feany, M.B. and Buckley, K.M. (1993a) The synaptic vesicle protein synaptotagmin promotes formation of filopodia in fibroblasts. Nature, 364, 537-540.

Feany, M.B., Yee, A.G., Delvy, M.L. and Buckley, K.M. (1993b) The synaptic vesicle proteins SV2, synaptotagmin and synaptophysin are sorted to separate cellular compartments in $\mathrm{CHO}$ fibroblasts. J Cell Biol, 123, 575-584.

Fergestad, T. and Broadie, K. (2001) Interaction of stoned and synaptotagmin in synaptic vesicle endocytosis. J Neurosci, 21, 1218-1227.

Fergestad, T., Davis, W.S. and Broadie, K. (1999) The stoned proteins regulate synaptic 
vesicle recycling in the presynaptic terminal. $J$ Neurosci, 19, 5847-5860.

Fernandez, I., Arac, D., Ubach, J., Gerber, S.H., Shin, O., Gao, Y., Anderson, R.G., Sudhof, T.C. and Rizo, J. (2001) Three-dimensional structure of the synaptotagmin 1 C2Bdomain: synaptotagmin 1 as a phospholipid binding machine. Neuron, 32, 1057-1069.

Fingerhut, A., von Figura, K. and Honing, S. (2001) Binding of AP2 to sorting signals is modulated by AP2 phosphorylation. J Biol Chem, 276, 5476-5482.

Ford, M.G., Mills, I.G., Peter, B.J., Vallis, Y., Praefcke, G.J., Evans, P.R. and McMahon, H.T. (2002) Curvature of clathrin-coated pits driven by epsin. Nature, 419, 361-366.

Fotin, A., Cheng, Y., Sliz, P., Grigorieff, N., Harrison, S.C., Kirchhausen, T. and Walz, T. (2004) Molecular model for a complete clathrin lattice from electron cryomicroscopy. Nature, 432, 573-579.

Fukuda, M. (2003) Synaptotagmins, $\mathrm{Ca}^{2+}$-and phospholipid-binding proteins that control $\mathrm{Ca}^{2+}$-regulated membrane tafficking. Recent Res. Devel. Chem. Phys. Lipids, 1, 1551.

Fukuda, M., Moreira, J.E., Liu, V., Sugimori, M., Mikoshiba, K. and Llinas, R.R. (2000) Role of the conserved WHXL motif in the $\mathrm{C}$ terminus of synaptotagmin in synaptic vesicle docking. Proc Natl Acad Sci U S A, 97, 14715-14719.

Gad, H. (2000) Synaptic vesicle endocytosis studied in a living synapse. Department of Neuroscience. Nobel Institute for Neurophysiology Karolinska Institutet, Stockholm, p. 48.

Galli, T. and Haucke, V. (2001) Cycling of synaptic vesicles: how far? How fast! Sci STKE, 2001, RE1.

Galli, T. and Haucke, V. (2004) Cycling of synaptic vesicles: how far? How fast! Sci STKE, 2004, re19.

Geppert, M., Goda, Y., Hammer, R.E., Li, C., Rosahl, T.W., Stevens, C.F. and Sudhof, T.C. (1994) Synaptotagmin I: a major Ca2+ sensor for transmitter release at a central synapse. Cell, 79, 717-727.

Grass, I., Thiel, S., Honing, S. and Haucke, V. (2004) Recognition of a Basic AP-2 Binding Motif within the C2B Domain of Synaptotagmin Is Dependent on Multimerization. $J$ Biol Chem, 279, 54872-54880.

Grigliatti, T.A., Hall, L., Rosenbluth, R. and Suzuki, D.T. (1973) Temperature-sensitive mutations in Drosophila melanogaster. XIV. A selection of immobile adults. Mol Gen Genet, 120, 107-114.

Gundelfinger, E.D., Kessels, M.M. and Qualmann, B. (2003) Temporal and spatial coordination of exocytosis and endocytosis. Nat Rev Mol Cell Biol, 4, 127-139.

Haffner, C., Di Paolo, G., Rosenthal, J.A. and de Camilli, P. (2000) Direct interaction of the $170 \mathrm{kDa}$ isoform of synaptojanin 1 with clathrin and with the clathrin adaptor AP-2. Curr Biol, 10, 471-474.

Han, W., Rhee, J.S., Maximov, A., Lao, Y., Mashimo, T., Rosenmund, C. and Sudhof, T.C. $(2004) \mathrm{N}$-glycosylation is essential for vesicular targeting of synaptotagmin 1 . Neuron, 41, 85-99. 
Haucke, V. and De Camilli, P. (1999) AP-2 recruitment to synaptotagmin stimulated by tyrosine-based endocytic motifs. Science, 285, 1268-1271.

Haucke, V. and Krauss, M. (2002) Tyrosine-based endocytic motifs stimulate oligomerization of AP-2 adaptor complexes. Eur J Cell Biol, 81, 647-653.

Haucke, V., Wenk, M.R., Chapman, E.R., Farsad, K. and De Camilli, P. (2000) Dual interaction of synaptotagmin with mu2- and alpha-adaptin facilitates clathrin-coated pit nucleation. Embo J, 19, 6011-6019.

Heldwein, E.E., Macia, E., Wang, J., Yin, H.L., Kirchhausen, T. and Harrison, S.C. (2004) Crystal structure of the clathrin adaptor protein 1 core. Proc Natl Acad Sci U S A, 101, 14108-14113.

Heuser, J.E. and Keen, J. (1998) Deep-etch visualization of proteins involved in clathrin assembly. J Cell Biol, 107, 877-886.

Hinrichsen, L., Harborth, J., Andrees, L., Weber, K. and Ungewickell, E.J. (2003) Effect of clathrin heavy chain- and alpha-adaptin-specific small inhibitory RNAs on endocytic accessory proteins and receptor trafficking in HeLa cells. J Biol Chem, 278, 4516045170

Honing, S., Ricotta, D., Krauss, M., Spate, K., Spolaore, B., Motley, A., Robinson, M., Robinson, C., Haucke, V. and Owen, D.J. (2005) Phosphatidylinositol-(4,5)bisphosphate regulates sorting signal recognition by the clathrin-associated adaptor complex AP2. Mol Cell, 18, 519-531.

Hui, E., Bai, J., Wang,P., Sugimori, M., Llinas, R.R. and Chapman, E.R. (2005) Three distinct kinetic groupings of the synaptotagmin family: candidate sensors for rapid and delayed exocytosis. Proc Natl Acad Sci U S A, 102, 5210-5214.

lezzi, M., Kouri, G., Fukuda, M. and Wollheim, C.B. (2004) Synaptotagmin V and IX isoforms control Ca2+-dependent insulin exocytosis. J Cell Sci, 117, 3119-3127.

Jackson, A.P., Flett, A., Smythe, C., Hufton, L., Wettey, F.R. and Smythe, E. (2003) Clathrin promotes incorporation of cargo into coated pits by activation of the AP2 adaptor micro2 kinase. J Cell Biol, 163, 231-236.

Jarousse, N. and Kelly, R.B. (2001a) The AP2 binding site of synaptotagmin 1 is not an internalization signal but a regulator of endocytosis. J Cell Biol, 154, 857-866.

Jarousse, N. and Kelly, R.B. (2001b) Endocytotic mechanisms in synapses. Curr Opin Cell Biol, 13, 461-469.

Jarousse, N., Wilson, J.D., Arac, D., Rizo, J. and Kelly, R.B. (2003) Endocytosis of synaptotagmin 1 is mediated by a novel, tryptophan-containing motif. Traffic, 4, 468478.

Jha, A., Agostinelli, N.R., Mishra, S.K., Keyel, P.A., Hawryluk, M.J. and Traub, L.M. (2004) A novel AP-2 adaptor interaction motif initially identified in the long-splice isoform of synaptojanin 1, SJ170. J Biol Chem, 279, 2281-2290.

Johannes, L. and Lamaze, C. (2002) Clathrin-dependent or not: is it still the question? Traffic, 3, 443-451. 
Keen, J.H., Willingham, M.C. and Pastan, I.H. (1979) Clathrin-coated vesicles: isolation, dissociation and factor-dependent reassociation of clathrin baskets. Cell, 16, 303312.

Kelly, L.E. and Phillips, A.M. (2005) Molecular and genetic characterisation of the interactions between the Drosophila stoned-B protein and AND DAP-160 (Intersectin). Biochem J.

Krauss, M., Kinuta, M., Wenk, M.R., De Camilli, P., Takei, K. and Haucke, V. (2003) ARF6 stimulates clathrin/AP-2 recruitment to synaptic membranes by activating phosphatidylinositol phosphate kinase type Igamma. J Cell Biol, 162, 113-124.

Llinas, R.R., Sugimori, M., Moran, K.A., Moreira, J.E. and Fukuda, M. (2004) Vesicular reuptake inhibition by a synaptotagmin I C2B domain antibody at the squid giant synapse. Proc Natl Acad Sci U S A, 101, 17855-17860.

Lodish, H., Berk, A., Zipursky, S.L., Matsudaira, P., Baltimore, D. and Darnell, J.E. (2000) Molecular Cell Biology. W.H.Freeman \& Co., New York.

Mackler, J.M., Drummond, J.A., Loewen, C.A., Robinson, I.M. and Reist, N.E. (2002) The $\mathrm{C}(2) \mathrm{B} \mathrm{Ca}(2+)$-binding motif of synaptotagmin is required for synaptic transmission in vivo. Nature, 418, 340-344.

Martina, J.A., Bonangelino, C.J., Aguilar, R.C. and Bonifacino, J.S. (2001) Stonin 2: an adaptor-like protein that interacts with components of the endocytic machinery. $J$ Cell Biol, 153, 1111-1120.

Matthias, K., Kirchhoff, F., Seifert, G., Huttmann, K., Matyash, M., Kettenmann, H. and Steinhauser, C. (2003) Segregated expression of AMPA-type glutamate receptors and glutamate transporters defines distinct astrocyte populations in the mouse hippocampus. J Neurosci, 23, 1750-1758.

Maycox, P.R., Link, E., Reetz, A., Morris, S.A. and Jahn, R. (1992) Clathrin-coated vesicles in nervous tissue are involved primarily in synaptic vesicle recycling. J Cell Biol, 118, 1379-1388.

McMahon, H.T. and Mills, I.G. (2004) COP and clathrin-coated vesicle budding: different pathways, common approaches. Curr Opin Cell Biol, 16, 379-391.

Miesenbock, G., De Angelis, D.A. and Rothman, J.E. (1998) Visualizing secretion and synaptic transmission with $\mathrm{pH}-$ sensitive green fluorescent proteins. Nature, 394, $192-$ 195.

Mishra, S.K., Hawryluk, M.J., Brett, T.J., Keyel, P.A., Dupin, A.L., Jha, A., Heuser, J.E., Fremont, D.H. and Traub, L.M. (2004) Dual engagement regulation of protein interactions with the AP-2 adaptor alpha appendage. J Biol Chem, 279, 46191-46203.

Mishra, S.K., Keyel, P.A., Edeling, M.A., Dupin, A.L., Owen, D.J. and Traub, L.M. (2005) Functional dissection of an AP-2 beta 2 appendage-binding sequence within the autosomal recessive hypercholesterolemia (ARH) protein. J Biol Chem.

Morgan, J.R., Prasad, K., Jin, S., Augustine, G.J. and Lafer, E.M. (2001) Uncoating of clathrin-coated vesicles in presynaptic terminals: roles for Hsc70 and auxilin. Neuron, 32, 289-300. 
Motley, A., Bright, N.A., Seaman, M.N. and Robinson, M.S. (2003) Clathrin-mediated endocytosis in AP-2-depleted cells. J Cell Biol, 162, 909-918.

Munro, S. (2004) Organelle identity and the organization of membrane traffic. Nat Cell Biol, 6, 469-472.

Nathke, I.S., Heuser, J., Lupas, A., Stock, J., Turck, C.W. and Brodsky, F.M. (1992) Folding and trimerization of clathrin subunits at the triskelion hub. Cell, 68, 899-910.

Nesterov, A., Carter, R.E., Sorkina, T., Gill, G.N. and Sorkin, A. (1999) Inhibition of the receptor-binding function of clathrin adaptor protein AP-2 by dominant-negative mutant mu2 subunit and its effects on endocytosis. Embo J, 18, 2489-2499.

Nicholson-Tomishima, K. and Ryan, T.A. (2004) Kinetic efficiency of endocytosis at mammalian CNS synapses requires synaptotagmin I. Proc Natl Acad Sci U S A, 101, 16648-16652.

Olusanya, O., Andrews, P.D., Swedlow, J.R. and Smythe, E. (2001) Phosphorylation of threonine 156 of the mu2 subunit of the AP2 complex is essential for endocytosis in vitro and in vivo. Curr Biol, 11, 896-900.

Owen, D.J., Collins, B.M. and Evans, P.R. (2004) Adaptors for clathrin coats: structure and function. Annu Rev Cell Dev Biol, 20, 153-191.

Owen, D. and Evans, P. (2003) The AP2 endocytic adaptor up close and personal: Mickey Mouse or Mr Tickle. the ELSO gazette, 13, 1-8.

Paoluzi, S., Castagnoli, L., Lauro, I., Salcini, A.E., Coda, L., Fre, S., Confalonieri, S., Pelicci, P.G., Di Fiore, P.P. and Cesareni, G. (1998) Recognition specificity of individual EH domains of mammals and yeast. Embo J, 17, 6541-6550.

Pearse, B.M. (1975) Coated vesicles from pig brain: purification and biochemical characterization. J Mol Biol, 97, 93-98.

Perry, M.M. and Gilbert, A.B. (1979) Yolk transport in the ovarian follicle of the hen (Gallus domesticus): lipoprotein-like particles at the periphery of the oocyte in the rapid growth phase. J Cell Sci, 39, 257-272.

Petrovich, T.Z., Merakovsky, J. and Kelly, L.E. (1993) A genetic analysis of the stoned locus and its interaction with dunce, shibire and Suppressor of stoned variants of Drosophila melanogaster. Genetics, 133, 955-965.

Phillips, A.M., Smith, M., Ramaswami, M. and Kelly, L.E. (2000) The products of the Drosophila stoned locus interact with synaptic vesicles via synaptotagmin. $J$ Neurosci, 20, 8254-8261.

Poskanzer, K.E., Marek, K.W., Sweeney, S.T. and Davis, G.W. (2003) Synaptotagmin I is necessary for compensatory synaptic vesicle endocytosis in vivo. Nature, 426, 559563.

Praefcke, G.J., Ford, M.G., Schmid, E.M., Olesen, L.E., Gallop, J.L., Peak-Chew, S.Y., Vallis, Y., Babu, M.M., Mills, I.G. and McMahon, H.T. (2004) Evolving nature of the AP2 alpha-appendage hub during clathrin-coated vesicle endocytosis. Embo J, 23, 4371-4383.

Richmond, J.E. and Broadie, K.S. (2002) The synaptic vesicle cycle: exocytosis and 
endocytosis in Drosophila and C. elegans. Curr Opin Neurobiol, 12, 499-507.

Ricotta, D., Conner, S.D., Schmid, S.L., von Figura, K. and Honing, S. (2002)

Phosphorylation of the AP2 mu subunit by AAK1 mediates high affinity binding to membrane protein sorting signals. J Cell Biol, 156, 791-795.

Ritter, B., Denisov, A.Y., Philie, J., Deprez, C., Tung, E.C., Gehring, K. and McPherson, P.S. (2004) Two WXXF-based motifs in NECAPs define the specificity of accessory protein binding to AP-1 and AP-2. Embo J, 23, 3701-3710.

Ritter, B., Philie, J., Girard, M., Tung, E.C., Blondeau, F. and McPherson, P.S. (2003) Identification of a family of endocytic proteins that define a new alpha-adaptin earbinding motif. EMBO Rep, 4, 1089-1095.

Robinson, M.S. and Bonifacino, J.S. (2001) Adaptor-related proteins. Curr Opin Cell Biol, 13, 444-453.

Rohde, G., Wenzel, D. and Haucke, V. (2002) A phosphatidylinositol (4,5)-bisphosphate binding site within mu2-adaptin regulates clathrin-mediated endocytosis. J Cell Biol, 158, 209-214.

Rohde, G., Wenzel, D. and Haucke, V. (2002) A phosphatidylinositol (4,5)-bisphosphate binding site within mu2-adaptin regulates clathrin-mediated endocytosis. J Cell Biol, 158, 209-214.

Rosenthal, J.A., Chen, H., Slepnev, V.I., Pellegrini, L., Salcini, A.E., Di Fiore, P.P. and De Camilli, P. (1999) The epsins define a family of proteins that interact with components of the clathrin coat and contain a new protein module. J Biol Chem, 274, 3395933965.

Roth, T.F. and Porter, K.R. (1964) Yolk Protein Uptake in the Oocyte of the Mosquito Aedes Aegypti. L. J Cell Biol, 20, 313-332.

Royle, S.J., Bobanovic, L.K. and Murrell-Lagnado, R.D. (2002) Identification of a noncanonical tyrosine-based endocytic motif in an ionotropic receptor. J Biol Chem, 277, 35378-35385.

Sambrook, J. and Russell, D.W. (2001) Molecular Cloning: A Laboratory Manual. CSHL Press, New York.

Schmid, S.L. (1997) Clathrin-coated vesicle formation and protein sorting: an integrated process. Annu Rev Biochem, 66, 511-548.

Schoch, S., Deak, F., Konigstorfer, A., Mozhayeva, M., Sara, Y., Sudhof, T.C. and Kavalali, E.T. (2001) SNARE function analyzed in synaptobrevin/VAMP knockout mice. Science, 294, 1117-1122.

Schwarz, T.L. (2004) Synaptotagmin promotes both vesicle fusion and recycling. Proc Natl Acad Sci U S A, 101, 16401-16402.

Sigismund, S., Woelk, T., Puri, C., Maspero, E., Tacchetti, C., Transidico, P., Di Fiore, P.P. and Polo, S. (2005) Clathrin-independent endocytosis of ubiquitinated cargos. Proc Natl Acad Sci U S A, 102, 2760-2765.

Slepnev, V.I. and De Camilli, P. (2000) Accessory factors in clathrin-dependent synaptic vesicle endocytosis. Nat Rev Neurosci, 1, 161-172. 
Smith, C.J., Grigorieff, N. and Pearse, B.M. (1998) Clathrin coats at 21 A resolution: a cellular assembly designed to recycle multiple membrane receptors. Embo J, 17, 4943-4953.

Sorkina, T., Bild, A., Tebar, F. and Sorkin, A. (1999) Clathrin, adaptors and eps15 in endosomes containing activated epidermal growth factor receptors. J Cell Sci, 112 ( Pt 3), 317-327.

Stimson, D.T., Estes, P.S., Rao, S., Krishnan, K.S., Kelly, L.E. and Ramaswami, M. (2001) Drosophila stoned proteins regulate the rate and fidelity of synaptic vesicle internalization. J Neurosci, 21, 3034-3044.

Stimson, D.T., Estes, P.S., Smith, M., Kelly, L.E. and Ramaswami, M. (1998) A product of the Drosophila stoned locus regulates neurotransmitter release. J Neurosci, 18, 96389649.

Sudhof, T.C. (2002) Synaptotagmins: why so many? J Biol Chem, 277, 7629-7632.

Sudhof, T.C. (2004) The synaptic vesicle cycle. Annu Rev Neurosci, 27, 509-547.

Sugita, S., Han, W., Butz, S., Liu, X., Fernandez-Chacon, R., Lao, Y. and Sudhof, T.C. (2001) Synaptotagmin VII as a plasma membrane $\mathrm{Ca}(2+)$ sensor in exocytosis. Neuron, 30, 459-473.

Takei, K. and Haucke, V. (2001) Clathrin-mediated endocytosis: membrane factors pull the trigger. Trends Cell Biol, 11, 385-391.

Takei, K., McPherson, P.S., Schmid, S.L. and De Camilli, P. (1995) Tubular membrane invaginations coated by dynamin rings are induced by GTP-gamma $S$ in nerve terminals. Nature, 374, 186-190.

Traub, L.M. (2003) Sorting it out: AP-2 and alternate clathrin adaptors in endocytic cargo selection. J Cell Biol, 163, 203-208.

Ungewickell, E. and Branton, D. (1981) Assembly units of clathrin coats. Nature, 289, 420422.

Upadhyaya, A.B., Lee, S.H. and DeJong, J. (1999) Identification of a general transcription factor TFIIAalpha/beta homolog selectively expressed in testis. J Biol Chem, 274, 18040-18048.

van der Weyden, L., Adams, D.J. and Bradley, A. (2002) Tools for targeted manipulation of the mouse genome. Physiol Genomics, 11, 133-164.

Walther, K., Diril, M.K., Jung, N. and Haucke, V. (2004) Functional dissection of the interactions of stonin 2 with the adaptor complex AP-2 and synaptotagmin. Proc Natl Acad Sci U S A, 101, 964-969.

Walther, K., Krauss, M., Diril, M.K., Lemke, S., Ricotta, D., Honing, S., Kaiser, S. and Haucke, V. (2001) Human stoned B interacts with AP-2 and synaptotagmin and facilitates clathrin-coated vesicle uncoating. EMBO Rep, 2, 634-640.

Warren, R.A., Green, F.A. and Enns, C.A. (1997) Saturation of the endocytic pathway for the transferrin receptor does not affect the endocytosis of the epidermal growth factor receptor. J Biol Chem, 272, 2116-2121. 
Wernick, N.L., Haucke, V. and Simister, N.E. (2004) Recognition of the tryptophan-based endocytosis signal in the neonatal Fc receptor by the mu subunit of AP-2. J Biol Chem.

Wilhelm, A., Volknandt, W., Langer, D., Nolte, C., Kettenmann, H. and Zimmermann, H. (2004) Localization of SNARE proteins and secretory organelle proteins in astrocytes in vitro and in situ. Neurosci Res, 48, 249-257.

Willnow, T.E. and Herz, J. (1994) Homologous ecombination for gene replacement in mouse cell lines. Methods in Cell Biology, 43, 305-333.

Zhang, B. (2003) Genetic and molecular analysis of synaptic vesicle recycling in Drosophila. J Neurocytol, 32, 567-589.

Zhang, J.Z., Davletov, B.A., Sudhof, T.C. and Anderson, R.G. (1994) Synaptotagmin I is a high affinity receptor for clathrin AP-2: implications for membrane recycling. Cell, 78, 751-760.

Zhang, Q., Fukuda, M., Van Bockstaele, E., Pascual, O. and Haydon, P.G. (2004) Synaptotagmin IV regulates glial glutamate release. Proc Natl Acad Sci U S A, 101, 9441-9446. 


\section{FIGURES AND TABLES FIGURES}

Figure 1.1 Diverse intracellular vesicular trafficking events in eukaryotic cells ............. 2

Figure 1.2 Routes of entry into eukaryotic cells........................................................... 3

Figure 1.3 Sequence of events during clathrin mediated endocytosis ......................... 6

Figure 1.4 Molecular architecture and organization of the clathrin coat........................ 8

Figure 1.5 Functional and molecular organization of heterotetrameric adaptor

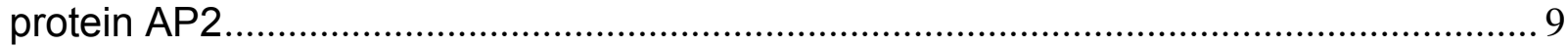

Figure 1.6 Domain organization of dynamin and effect GTPgS on its function........... 10

Figure 1.7 Domain and motif organization of mammalian intersectin 1 and eps 15

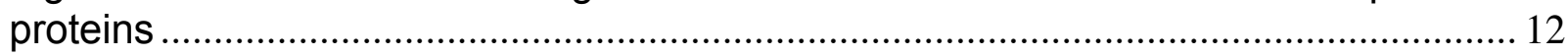

Figure 1.8 Synaptic vesicle cycle ........................................................................... 13

Figure 1.9 Synaptic vesicle proteins and models for synaptic vesicle cycling............. 14

Figure 1.10 Classification and exon/intron organization of human synaptotagmin

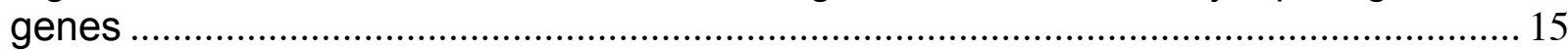

Figure 1.11 Molecular morphology of synaptotagmins and C2B domain structure ..... 16

Figure 1.12 Phenotypic analyis of the Drosophila stoned mutants.............................. 19

Figure 1.13 Orthologues of stoned genes from selected species.............................. 21

Figure 1.14 Multiple sequence alignment of stonins .............................................. 23

Figure 1.15 Genomic organization of the mouse stonin 1 locus .................................. 24

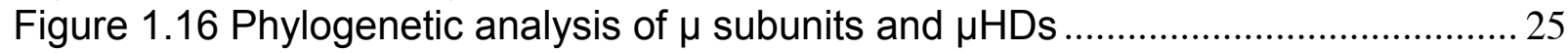

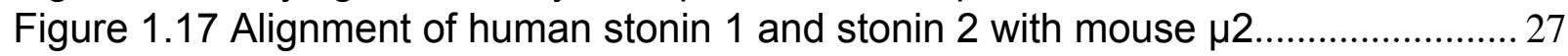

Figure 2.1 Testing of stonin 2 antisera .................................................................. 41

Figure 2.2 Testing of stonin1 antisera by immunofluorescence microscopy ............... 42

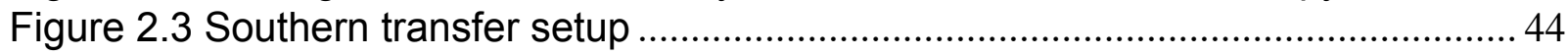

Figure 2.4 Setup for electric field assisted Western blotting ....................................... 47

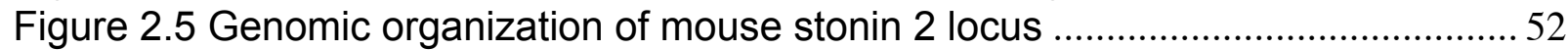

Figure 2.6 Confirmation of cosmid clones by Southern hybridization .......................... 53

Figure 2.7 Cloning of the targeting construct............................................................ 54

Figure 2.8 Targeting strategy for mouse stonin 2 genomic locus ............................... 54

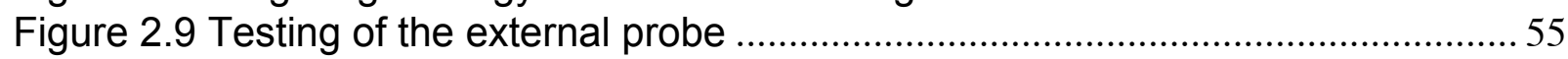

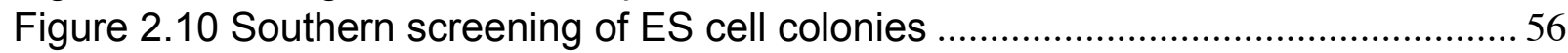

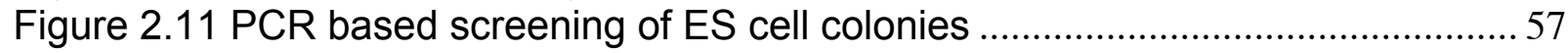

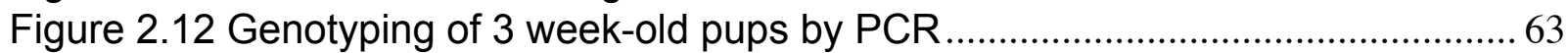

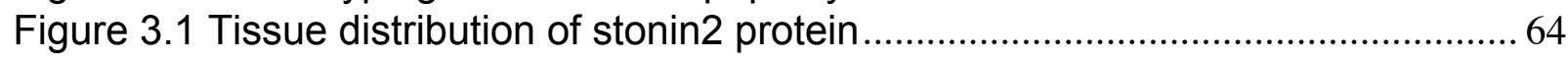

Figure 3.2 Expression of stonin2 in different brain regions ....................................... 65

Figure 3.3 Stonin 2 expression in different cell lines and primary cells........................ 66

Figure 3.4 Endogenous stonin 2 expression in neurons............................................ 66

Figure 3.5 Affinity purification of Eps15 and Intersectin1 using GST-Stnonin2NT

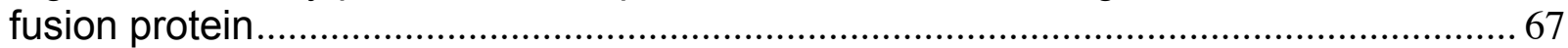

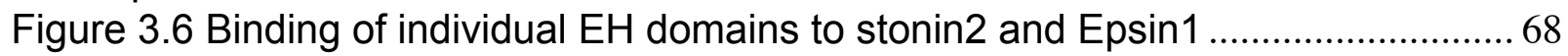

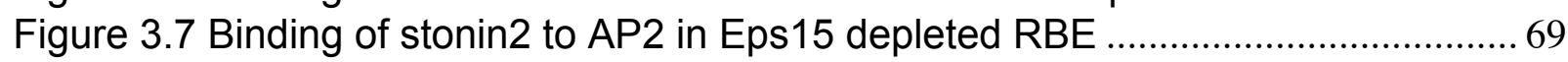

Figure 3.8 Interaction of stonin1 with AP2, Eps15 and intersectin1 ........................... 70

Figure 3.9 Novel AP2 interaction motif initially discovered in stonins ........................ 71

Figure 3.10 Delineation of WvXF type motifs in stonin1 sequence ............................ 71

Figure 3.11 Overexpression of EGFP-stonin2 but not EGFP-stonin1 causes a

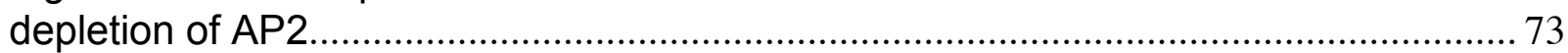

Figure 3.12 Overexpression of EGFP-stonin 2 but not EGFP-stonin 1 inhibits endocytic uptake of Texas-Red labeled transferrin. 
Figure 3.13 Binding of stonin2 to synaptotagmin1 and AP2 …................................ 75

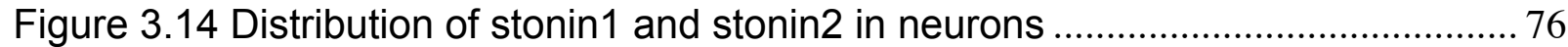

Figure 3.15 Translocation of stonin 2 to the plasmalemma in N1E cells .................... 77

Figure 3.16 In vivo interaction assays for stonin2 and synaptotagmins $1-11 \ldots \ldots \ldots \ldots . . .79$

Figure 3.17 In vivo interaction assays for stonin1 and synaptotagmins $1-11 \ldots \ldots \ldots \ldots . . . .80$

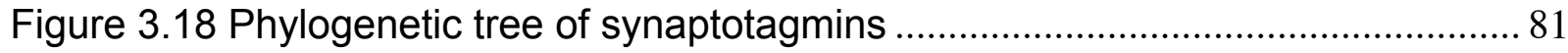

Figure 3.19 Translocation of stonin2 to AP2 coated pits in N1E cells ......................... 82

Figure 3.20 Stonin 2 facilitates endocytosis of plasmalemma stranded

synaptotagmin 1 in an AP2 dependent manner ............................................................. 83

Figure 3.21 Stonin2 facilitates endocytosis of synaptotagmin 2 but not

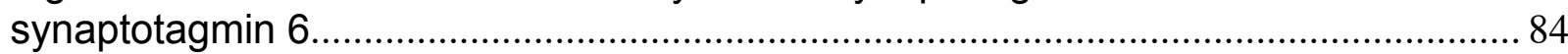

Figure 3.22 Quantification of synaptotagmin 1 internalization by various stonin 2

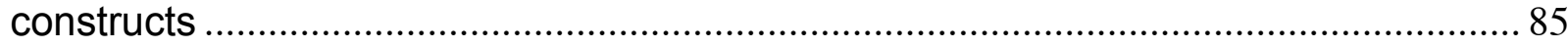

Figure 3.23 Co-Immunoprecipitation of synaptotagmin 1 only in presence of

stonin2

Figure 3.24 Incorporation the stonin $2-\mu \mathrm{HD}$ into the AP2 complex facilitates

synaptotagmin 1

Figure 3.25 RNAi mediated knock-down AP2- $\mu 2$ in HEK cells causes depletion of

AP2-coated pits and an inhibition of transferrin uptake

Figure 3.26 Stonin2 cannot facilitate internalization of synaptotagmin1 in the

absence of AP2.

Figure 3.27 AP2 knock-down also inhibits endocytosis of synaptotagmin 1 in the

absence of exogenously expressed stonin 2 .............................................................. 90

Figure 3.28 Stonin2 C-terminal truncations and synaptotagmin 1 endocytosis ............ 92

Figure 3.29 Internalized synaptotagmin 1 is sorted to early endosomes ..................... 94

Figure 3.30 Colocalization of internalized synaptotagmin 1 with AP2 ........................ 96

Figure 3.31 Stonin 2 facilitates endocytic internalization of synaptotagmin 1 and its

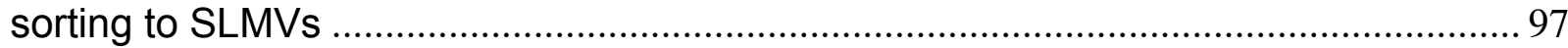

Figure 3.32 Western blot analysis of stonin 2 knock-out ......................................... 99

Figure 3.33 Quantitative western blot analysis of protein expression levels in stonin

2 animals

Figure 3.34 Subcellular distribution of synaptotagmin 1 and other proteins in wild-

type and knock-out mice brain extracts

Figure 3.35 Synaptotagmin 1 immunostaining and localization in stonin 2 wild-type

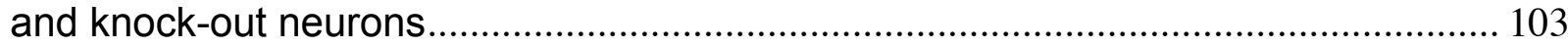

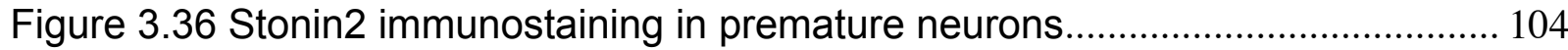

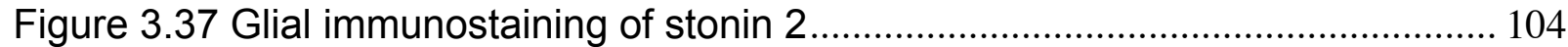

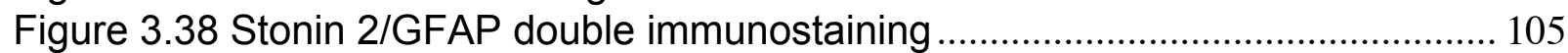

Figure 3.39 Colocalization of stonin2 and AP2 in glial cells .................................... 106

Figure 3.40 Expression and distribution pattern of stonin2 in U343 cells ................... 106

Figure 3.41 Localization of exogenously expressed EGFP-stonin 1 and stonin 2 in

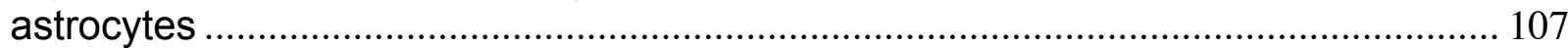

Figure 3.42 Colocalization of transfected pcHA-Stonin $2{ }^{\partial 420}$ with AP2 in astrocytes.. 107

Figure 3.43 Immunolocalization of endogenous stonin 1 and stonin 2 in astrocytes. 108 Figure 3.44 Colocalization of Eps15 and intersectin1 to plasma membrane coated

pit clusters

Figure 4.1 Crystal structure of $\alpha$-adaptin appendage domain in complex with

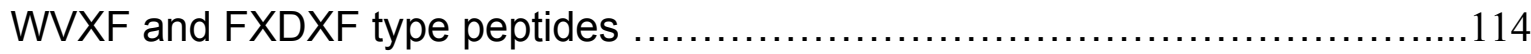




\section{TABLES}

Table 1.1 Close syntheny of human stonin 1 and stonin 2 gene loci ......................... 23

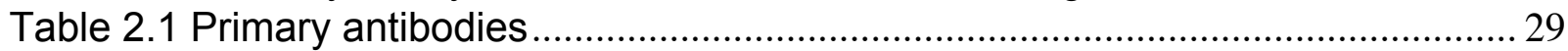

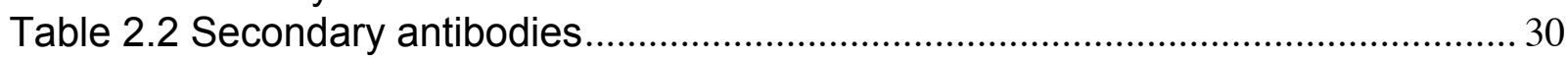

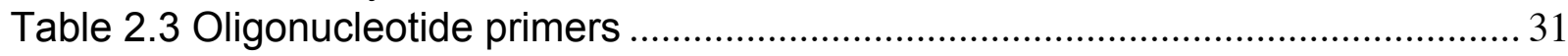

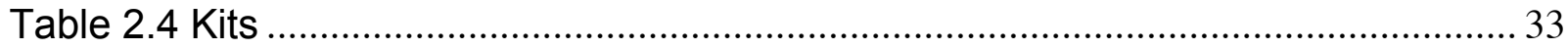

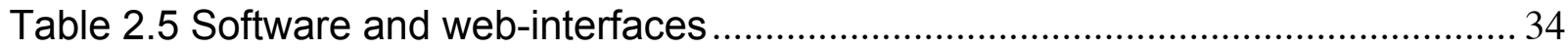

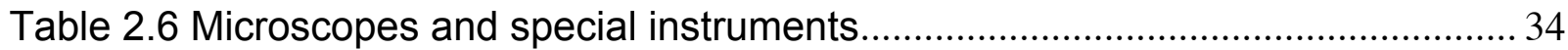

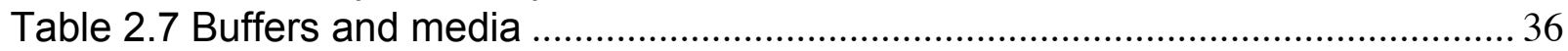

Table 2.8 Preparation of SDS gels .......................................................................... 46

Table 2.9 Composition of the ES culture media ........................................................ 48

Table 3.1 Effect of different stonin constructs on synaptotagmin1 endocytosis.......... 93

Table 3.2 Genotypes of the offspring as a result of stonin 2 heterozygote crosses .. 100

Table 4.1 Alternate sorting adaptors in CME ............................................................ 112 


\section{ABBREVIATIONS}

a

a.a.

AAK 1

Al

AP

APS

Arf6

ATP

BBD

bp

BSA

$\mathrm{C}$

CCV

cDNA

CCP

$\mathrm{CCV}$

$\mathrm{CHC}$

CLC

CME

CNS

$\mathrm{CO}_{2}$

colP

CT

$\mathrm{ddH}_{2} \mathrm{O}$

DIV

DMEM

DMSO

DNA

dNTP

EDTA

EEA1

EGF

EGFP

EH

EJC

EJP

ER

ERGIC

ES(C)

$\mathrm{EtBr}$

et al.

$\phi$

FCS

$\mathrm{g}$

GRF

GSDB

GST

GTP

$\mathrm{h}$ adenosine

amino acid

adaptor-associated kinase 1

antibody internalization

adaptor protein

ammonium persulfate

ADP rybosylation factor

adenosine triphosphate

beta-binding domain

basepair

bovine serum albumine

cytosine

clathrin-coated vesicle

complementary DNA

clathrin-coated pit

clathrin-coated vesicle

clathrin heavy chain

clathrin light chain

clathrin-mediated endocytosis

central nervous system

carbondioxide

coimmunoprecipitation

carboxy-terminal

distilled deionized water

days in vitro

Dulbecco's modified eagle medium

demethylsulfoxide

desoxyribonucleic acid

desoxyribonucleic acid triphosphate

ethylendiaminetetraacetate

early endosome antigen 1

epidermal growth factor

enhanced green fluorescence protein

eps15 homology

excitatory junctional current

excitatory junctional potential

endoplasmic reticulum

endoplasmic reticulum-Golgi intermediate compartment

embryonic stem (cell)

ethidium bromide

et alii

bulky hydrophobic amino acid residue

feotal calf serum

guanin

growth-hormone releasing factor

goat serum dilution buffer

glutathione-S-transferase

guanosin triphosphate

hour 


$\begin{array}{ll}\text { HA } & \text { hemeagglutinine } \\ \text { HEK 293 } & \text { human embryonic kidney } \\ \text { hsc 70 } & \text { heat shock chaperone } 70 \\ \text { IB } & \text { immunoblotting } \\ \text { IF } & \text { immunofluorescence } \\ \text { IL-2 } & \text { interleukin 2 } \\ \text { IP } & \text { immunoprecipitation } \\ \text { kb } & \text { kilobase } \\ \text { kDa } & \text { kilo Dalton } \\ \text { KO } & \text { knock-out } \\ \text { LB } & \text { Luria-Bertani } \\ \text { (L)DCV } & \text { (large) dense core vesicle } \\ \text { LDLR } & \text { low-density lipoprotein receptor } \\ \text { LIF } & \text { leukemia inhibitory factor } \\ \text { M } & \text { molar } \\ \text { mA } & \text { milliampere } \\ \text { MCS } & \text { multiple cloning site } \\ \text { min } & \text { minute } \\ \text { mI } & \text { mililiter } \\ \text { mM } & \text { millimolar } \\ \text { mRNA } & \text { messenger RNA } \\ \text { MW } & \text { molecular weight } \\ \text { NC } & \text { nitrocellulose } \\ \text { NCBI } & \text { National Center of Biotechnology Information } \\ \text { nm } & \text { nanometer } \\ \text { NMJ } & \text { neuromuscular junction } \\ \text { NT } & \text { amino-terminal } \\ \text { PAGE } & \text { plyacrylamide gel electrophoresis } \\ \text { PBS } & \text { phosphate buffered saline } \\ \text { PC 12 } & \text { pheochromocytoma } \\ \text { PCR } & \text { polymerase chain reaction } \\ \text { PFA } & \text { paraformaldehyde } \\ \text { PH } & \text { pleckstrin homology } \\ \text { pH } & \text { preponderance of hydrogene ions } \\ \text { PIP } 3 & \text { phosphatidylinositol-(3,4,5)-trisphosphate } \\ \text { PIP } & \text { phosphatidylinositol-(4,5)-bisphosphate } \\ \text { PIPKl } & \text { phosphatidylinositol phosphate kinase I gamma } \\ \text { PMEF } & \text { primary mouse embryonic fibroblast } \\ \text { PMSF } & \text { phenyl methyl sulfonyl fluoride } \\ \text { PRD } & \text { proline-rich domain } \\ \text { RBE } & \text { rat brain extract } \\ \text { RNA } & \text { ribonucleic acid } \\ \text { RNAi } & \text { RNA interference } \\ \text { rpm } & \text { rotations per minute } \\ \text { RT } & \text { room temperature } \\ \text { RT-PCR } & \text { reverse transcription-polymerase chain reaction } \\ \text { SH3 } & \text { src } 3 \text { homology } \\ \text { SLMV } & \text { synaptic like micro vesicles } \\ \text { SDS } & \text { sodium dodecyl sulfate } \\ \text { siRNA } & \text { small interferring RNA } \\ \text { Std. } & \text { standard } \\ & \end{array}$




$\begin{array}{ll}\text { StoHD } & \text { stonin-homology domain } \\ \text { SV } & \text { synaptic vesicle } \\ t & \text { thymine } \\ \text { Taq } & \text { Thermus aquaticus } \\ \text { TBS } & \text { tris buffered saline } \\ \text { TE } & \text { Tris-EDTA } \\ \text { TEMED } & \text { N,N,N,N-tetramethylethylendiamine } \\ \text { TfR } & \text { transferrin receptor } \\ \text { TGN } & \text { trans-Golgi network } \\ T_{m} & \text { melting temperature } \\ \text { Tris } & \text { Tris(hydroxymethyl)aminomethane } \\ U & \text { units } \\ \text { u } & \text { uracile } \\ \text { UV } & \text { ultraviolet } \\ X & \text { variable amino acid residue } \\ \text { WB } & \text { Western blot } \\ \text { WT } & \text { wild-type } \\ \mu l & \text { microliter } \\ \mu m & \text { micrometer } \\ \mu M & \text { micromolar } \\ \mu H D & \mu \text {-homology domain } \\ 2 x Y T & \text { two-fold yeast extract-tryptone medium }\end{array}$




\section{ACKNOWLEDGEMENTS}

This work would have been impossible without the contributions of many people who offered their help, guidance or at least sympathies during various phases of my project.

I thank my thesis supervisor Prof. Dr. Volker Haucke for giving me the opportunity to do research in his lab (which I truly enjoyed) and for his guidance throughout my experimental work. I thank him a second time for his contagious enthusiasm.

I thank Prof. Dr. Reinhard Jahn and Prof. Dr. Kurt von Figura, my thesis committee members, for co-supervising my project and giving invaluable advice during our meetings.

I thank my colleagues Kristin Walther and Nadja Jung for working closely together in harmony at our common projects. I also thank other members of my lab Arndt Pechstein, Claus Rodemer, Ingeborg Walther, Isabelle Grass, Junyong Jia, Kathrin Kastning, Michael Krauss, Michael Schmidt, Steffanie Thiel and Viktoria Kukhtina for the enjoyable working atmosphere. I thank Conni Podjaski for her excellent work during her time with us and for the in-situ experiments she performed quite independently.

I thank Dr. Peter Schu for his advices during the creation of the stonin 2 knock-out mice and Dr. Hermann Riedesel and his staff at Max-Planck Institute for Experimental Medicine for generation of stonin 2 chimeras.

I thank Dr. Steffen Burkhardt and the coordination office of the Molecular Biology Program for the excellent organization.

I thank my Turkish, German and international friends from the Molecular Biology and Neurosciences Programs for their wonderful company (that I surely miss a lot) during my days in Göttingen.

I am of course always grateful to my parent for their love and support.

I received a Lichtenberg stipend during the first three years of my project. 


\section{CURRICULUM VITAE}

\section{Personal Information}

Name

Date of birth

Place of birth

Nationality

Education

1984-1989

1989-1996

$1996-2000$

10.2000-08.2001

10.2001-06-2005
: Muhammed Kasım Diril

: 18.01.1978

: Çanakkale, Turkey

: Turkish
: Primary School, Eskisehir

: Eskisehir Anatolian High School, Eskisehir

: Bogazici University, Istanbul

Faculty of Arts and Sciences

B.Sc. degree in Molecular Biology and Genetics

: International M.Sc./Ph.D. Program 'Molecular Biology'

Georg-August University of Göttingen, Germany

Completion of the first phase of the program

: Second phase of the program

Ph.D. work in the laboratory of Prof. Volker Haucke Institute of Biochemistry, Free University of Berlin

Subject: Genetic analysis of stonin 2 function in vivo

\section{Contact Details}

Address

: Institut Chemie-Biochemie, FU Berlin

Takustr. 6 1.OG

$$
14195 \text { Berlin, Germany }
$$

Phone

: +493083856932

Permanent e-mail : kasimdiril@yahoo.com 


\section{PUBLICATIONS}

Walther, K., Krauss, M., Diril, M.K., Lemke, S., Ricotta, D., Honing, S., Kaiser, S. and Haucke, V. (2001) Human stoned B interacts with AP-2 and synaptotagmin and facilitates clathrin-coated vesicle uncoating. EMBO Rep, 2, 634-640.

Walther, K., Diril, M.K., Jung, N. and Haucke, V. (2004) Functional dissection of the interactions of stonin 2 with the adaptor complex AP-2 and synaptotagmin. Proc Natl Acad Sci U S A, 101, 964-969. 